UCRL-LR-117512

Distribution Category UC-700

\title{
DISCLAIMER
}

This report was prepared as an account of work sponsored by an agency of the United States Government. Neither the United States Government nor any agency thereof, nor any of their employees, makes any warranty, express or implied, or assumes any legal liability or responsibility for the accuracy, completeness, or usefulness of any information, apparatus, product, or process disclosed, or represents that its use would not infringe privately owned rights. Reference herein to any specific commercial product, process, or service by trade name, trademark, manufacturer, or otherwise does not necessarily constitute or imply its endorsement, recommendation, or favoring by the United States Government or any agency thereof. The views and opinions of authors expressed herein do not necessarily state or reflect those of the United States Government or any agency thereof.

\section{Reflectivity of Plasmas Created By High-Intensity, Ultra-Short Laser Pulses}

\author{
David Michael Gold \\ (Ph.D. Thesis)
}

June 1994

\section{LAWRENCE LIVERMORE NATIONAL LABORATORY University of California - Livermore, California $\bullet 94551$}

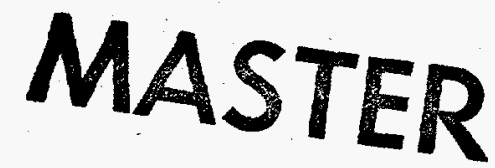




\section{DISCLAIMER}

Portions of this document may be illegible in electronic image products. Images are produced from the best available original document. 
Reflectivity of Plasmas Created By

High-Intensity, Ultra-Short Laser Pulses

By

David Michael Gold

B.S. (University of California, Davis) 1987

M.S. (University of California, Davis) 1988

DISSERTATION

Submitted in partial satisfaction of the requirements for the degree of

DOCTOR OF PHILOSOPHY

in

Engineering-Applied Science

in the

GRADUATE DIVISION

of the

UNIVERSITY OF CALIFORNIA

DAVIS

Approved:

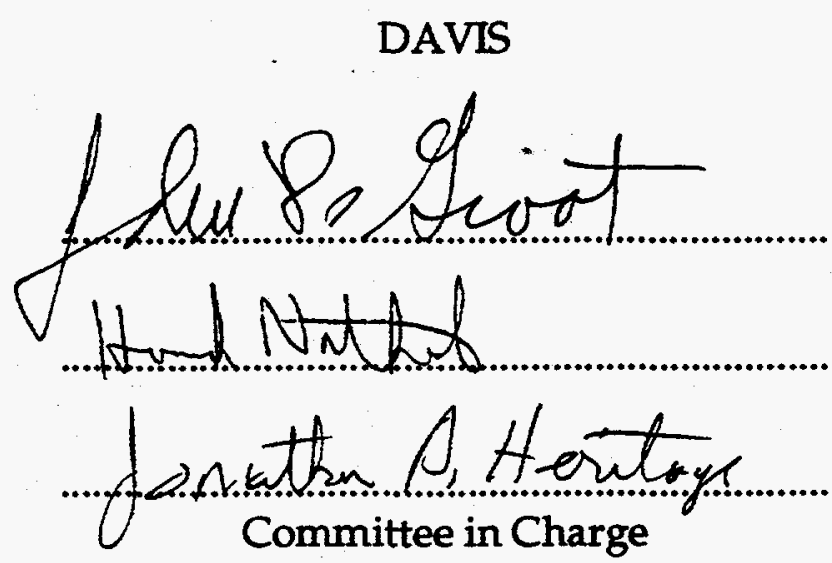

1994 


\author{
To Susan \\ Who's love reminds me eoery day \\ of the awe and wonder that physics is all about. \\ and \\ Who's patience and support were at least as important \\ to this dissertation as the laser was.
}

To My Family

Who taught me the value of education and the importance of common sense.

\title{
To My Friends
}

Who made these years not just bearable, but full of life. 


\section{ACKNOWLEDGMENTS}

Not only does a graduate student "stand on the shoulders of giants," but he often needs a ladder to get there. The following persons provided the ladder. Their contributions are gratefully acknowledged.

My dear wife, Susan, brought me happiness and perspective in these dark times. Her editing skills and gentle urging kept my writing on track, while her love, humor and joie de vivre kept my life on track.

My family instilled the work ethic and the wisdom to start down this path. Their contributions are everlasting.

Mike, Inés, Ying, Yang, and Barney taught me more during graduate school than I will ever fully realize. They deserve much of the credit for allaying the fears, destroying the self doubt, and rejecting the cynicism.

My thesis advisor, Dr. John DeGroot, was especially helpful and encouraging as the end drew near. My research advisor, Dr. Howard Nathel, gave me guidance and an unrestricted environment to learn in. Dr. John Heritage's reading of the manuscript was greatly appreciated.

Many "USP'ers" had a hand in the progress of my work. Bill White, Jim Hunter, David Fittinghoff, Dwight Price, Linn van Woerkom and James Norby were always available for experimental help. Their friendship and technical assistance were invaluable when I was a neophyte graduate student. Paul Bolton's advice was always accessible. It included a delight for physics, careful methods and dry humor which will not be forgotten. Ronnie Shepherd gave me many new ideas and considered every theory I proposed seriously, before trashing it with his wealth of plasma physics knowledge and infectious laugh.

Rosemary Walling and Britton Chang taught me the wonders and terrors of plasma simulations. Rosemary also gave invaluable advice and support in the final anxious months. Britton took great pains to make sure I understood the difference between a calculation and a theory.

The technical support at LLNL is second to none, so thanks goes out to all the technicians and staff who's name never appears on the experimental hardware.

And to all the Scottish dancers out there who kept me from atrophying here at the computer, the work is done -- it's Ceilidh time! 


\section{TABLE OF CONTENTS}

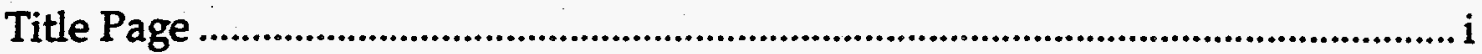

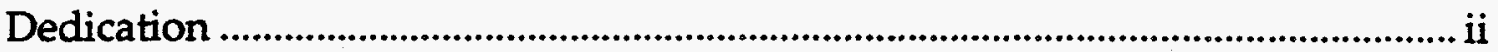

Acknowledgments ..............................................................................................................ii

Table of Contents....................................................................................................... iv

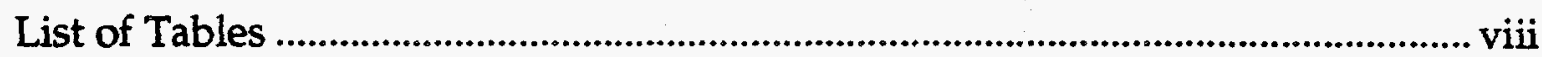

List of Figures........................................................................................................... ix

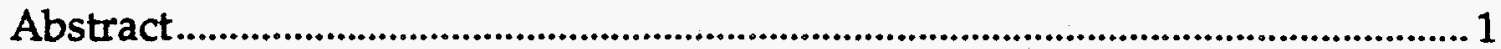

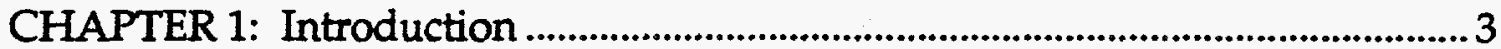

CHAPTER 2: The Amplified, Ultra-Short Pulse Laser System............................... 10

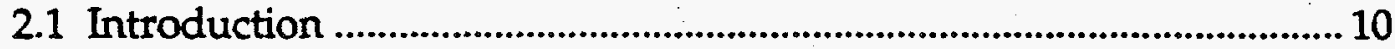

2.2 The Evolution of Amplified, Ultra-Short Pulse Lasers ......................... 12

2.3 Applications of Ultra-Short Pulse Lasers................................................ 18

2.4 Mode Locking Lasers ................................................................................ 20

2.4.1 Importance to High-Intensity Systems.......................................20

2.4.2 Mode Locking Basics ................................................................... 20

2.4.3 Active AM Mode Locking............................................................. 24

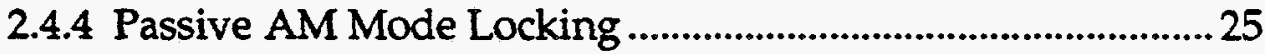

2.4.5 FM Mode Locking ............................................................................ 27

2.4.5 Other Methods of Producing Short Pulses ............................... 28

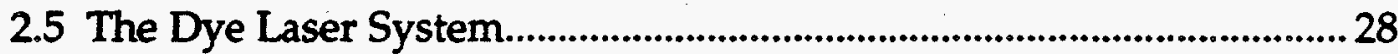

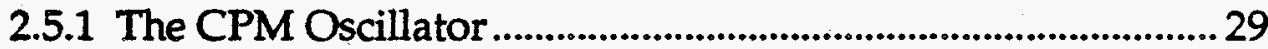

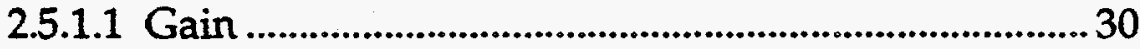

2.5.1.2 Saturable Absorber........................................................32

2.5.1.3 Dispersion Compensation in the CPM........................ 36

2.5.1.4 Pulse Propagation Around the Cavity ....................... 37 
2.5.1.6 Typical Operating Parameters. 38

2.5.2 The Argon Ion Oscillator Pump Laser 40

2.5.3 The Amplifier System ..................................................................... 41

2.5.3.1 Amplifier System Design and Layout........................ 41

2.5.3.2 Amplifier Analysis ........................................................4 47

2.5.3.3 Amplified Laser Output Characteristics.................... 48

2.5.4 The Nd:YAG Amplifier Pump Laser.......................................... 51

2.6 Measurement of Ultra-Short Pulses....................................................... 53

CHAPTER 3: An Introduction to Laser-Produced Plasmas...................................... 60

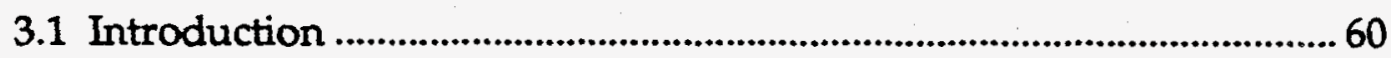

3.2 The Laser Plasma: Long Pulse and Short Pulse Plasmas.......................60

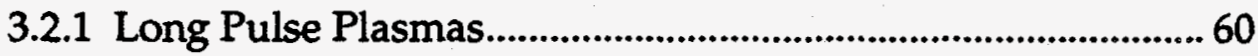

3.2.3 Short Pulse Plasmas ................................................................. 65

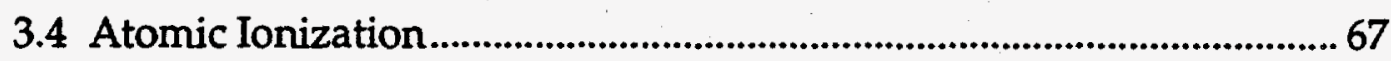

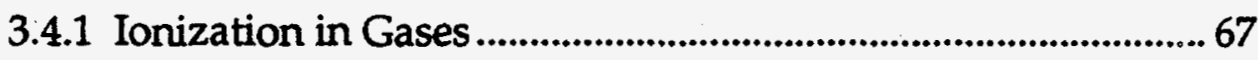

3.4.1.1 Multi-Photon and Tunneling Ionization..................... 67

3.4.1.2 Other Ionization Models ............................................... 72

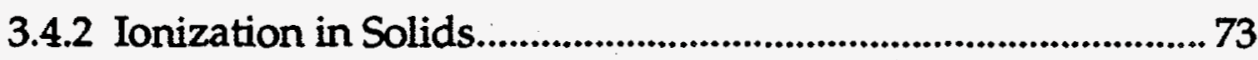

3.3 Ultra-Short Pulse Interaction With a Solid ............................................... 75

CHAPTER 4: Space- and Time-Integrated Reflectivity Measurements ................80

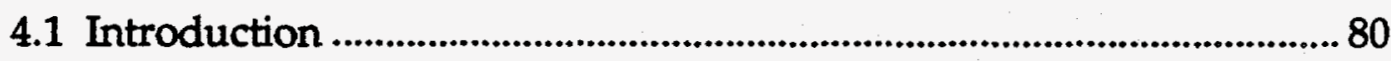

4.2 Self-Reflectivity Experimental Setup........................................................ 83

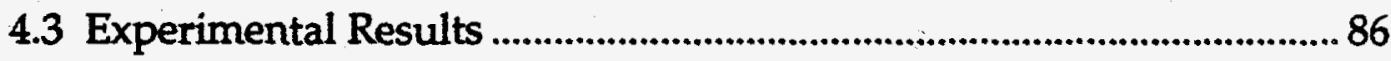

4.3.1 Energy Reflectivity Measurements ............................................... 86

4.3.2 Cold Target and High Prepulse Measurements ...................... 89

4.3.3 Analysis of Results: The Metals $\mathrm{Al}$ and $\mathrm{Au}$............................ 90

4.3.3.1 Analytic Calculations................................................91 
4.3.3.2 High-Field Effects............................................................ 100

4.3.3.3 Strongly-Coupled Plasma Effects .............................. 105

4.3.3.4 Numerical Simulation..................................................... 108

4.3.4 Analysis of Results: The Insulator $\mathrm{SiO}_{2} \ldots \ldots . . . \ldots \ldots . . . . . . . . . . . . . . . . . . .109$

4.4 A Nonequilibrium Plasma Model for Insulators.................................... 110

4.4.1 Basic Equations................................................................................ 110

4.4.2 Kinetic Coefficients ..................................................................... 112

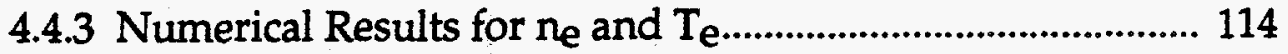

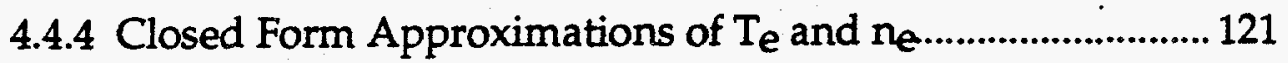

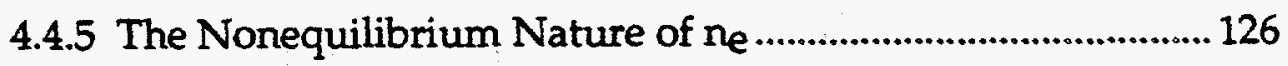

4.4.6 The Self-Induced Reflection of a Laser Pulse......................... 129

CHAPTER 5: Space- and Time-Resolved Reflectivity Measurements ................. 133

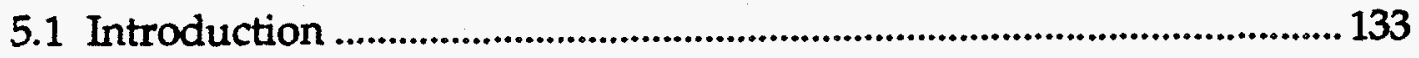

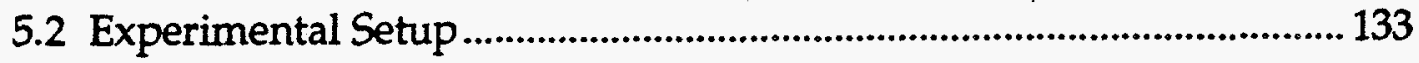

5.2.1 Pump-Probe Design .................................................................... 133

5.2.2 Absolute Calibration Measurements....................................... 137

5.2.3 Spatial Imaging System ............................................................. 139

5.3 Experimental Results and Discussion ...................................................... 141

5.3.1 Reflectivity, Transmission and Scatter Measurements .......... 141

5.3.2 Spatially-Resolved Reflectivity.................................................. 142

5.3.3 Electron Heat Conduction and Plasma

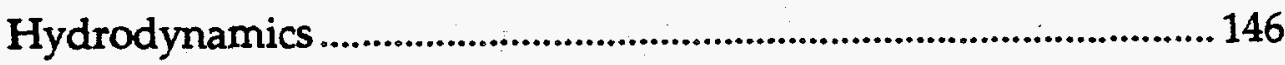

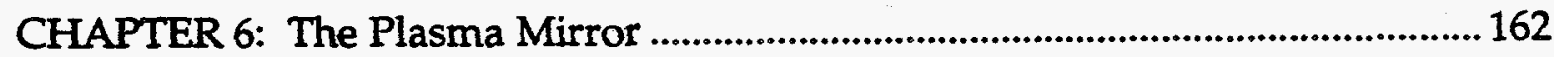

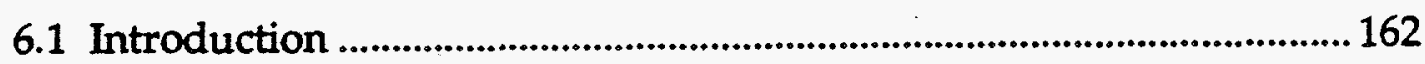

6.2 The Plasma Mirror Scheme ...................................................................... 166

6.3 Work Related to the Plasma Mirror........................................................... 170

6.4 The Solid Plasma Mirror Experiment........................................................... 172 
6.4.1 Reflectivity Measurements.......................................................... 175

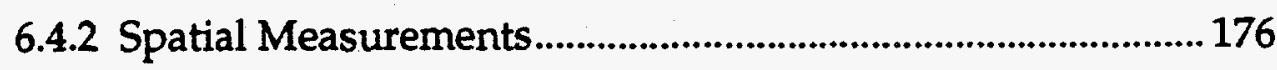

6.4.3 Temporal Measurements........................................................... 178

6.4.4 Prepulse Suppression Measurements ...................................... 180

6.5 The Liquid Plasma Mirror Experiment....................................................... 184

6.6 A Model for the Plasma Mirror ................................................................... 189

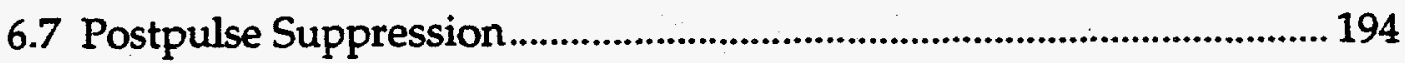

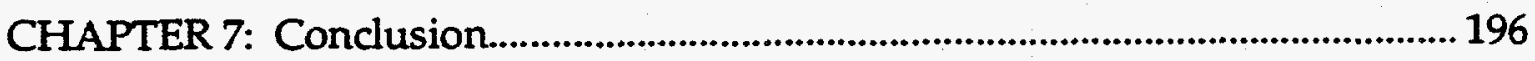

Appendix A: Rate Equations for Ion Production ................................................... 200

Appendix B: Laser-Assisted Avalanche Coefficients............................................. 202

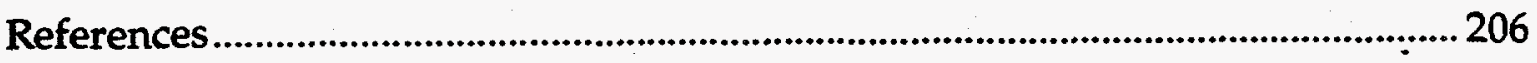




\section{LIST OF TABLES}

2.1: Pulsewidths achievable by mode locking in various laser media.

2.2: Correlation widths for three transform-limited pulse shapes. 56

3.1: Comparison of general plasma characteristics created by long ( $>1 p s)$ and short (<1ps) laser pulses.

4.1: Calculated and observed values of percentage absorption for $\mathrm{Al}$ at $100-120 \mathrm{eV}$ and $\mathrm{Au}$ at $150-170 \mathrm{eV}$.

6.1: Observed reflectivity and calculated contrast enhancement factor for a. $2.5 \mathrm{~mJ}, 140 \mathrm{fs}$ pulse as a function of angle of incidence, focal spot and polarization. 


\section{LIST OF FIGURES}

1.1: Ultra-short pulse lasers form the highest temperature-density laboratory plasmas and approach stellar interior states

2.1: New laser technology drives progress to intensity thresholds where new physical phenomena occur.

2.2: Bandwidth requirement for a transform-limited, $614 \mathrm{~nm}$, Gaussian pulse as a function of pulsewidth.

2.3: The field and intensity of a mode-locked laser with $\mathrm{N}=100$ modes phase-locked.

2.4: Gain and absorber saturation on each pass in a cw mode-locked laser. 26

2.5: Comparison of the CPM measured second harmonic generation autocorrelation signal with the predicted $\operatorname{sech}^{2}$ pulseshape.

2.6: The CPM laser oscillator.

2.7: The dye circulation system for the gain and saturable absorber dyes in the CPM dye laser oscillator.

2.8: Schematic energy level diagram and pumping cycle in R-6G dye.

2.9: Chirped pulse resulting from SPM

2.10: The 4-prism sequence in the CPM cavity compresses the pulse by forming a longer optical path for leading lower frequencies. Trailing higher frequencies catch up and linear chirp is removed. 
2.12: The CPM typically operates near $1.7 \times$ transform limit.

2.13: Energy levels and radiative pathways of the $4 \mathrm{p}-4 \mathrm{~s}$ argon ion laser transitions.

2.14: Schematic dye laser oscillator, amplifier, and pulse compression system.

2.15: The 6-pass bow-tie amplifier and Nd:YAG pump source 44

2.16: Relay imaging with a pinhole provides magnification, spatial filtering and image relaying.

2.17: The uniform illumination in the Bethune cell amplifier insured amplified pulses with good spatial quality even if the pump beam was not completely uniform.

2.18: The laser system linear dispersion was compensated by a grating pulse compressor.

2.19: The amplified pulse and spectrum. 49

2.20: High-dynamic-range amplified pulse autocorrelation at peak energy $>3 \mathrm{~mJ}$ 50

2.21: Energy levels for the Nd:YAG pump laser. 52

2.22: The unstable resonator and Q-switch of a Nd:YAG laser. 52

2.23: The rapid-scan (shaker), second-order autocorrelator. The slowscan, second-order autocorrelator. 
2.24: Pulse shape compared with fits to Gaussian, sech 2 , and Lorentzian curves.

2.25: The single-shot, second-order autocorrelator.

3.1: The critical density versus wavelength for most laser wavelengths.

3.2: Schematic electron density profile in a long pulse ( $>1 \mathrm{ps}$ ) laserproduced plasma, showing the location of major light-plasma coupling processes.

3.3: MPI (4 photon) picture of ionization of an atom. 69

3.4: Atomic potential distortion by a strong light field allows tunneling ionization to occur.

4.1: Schematic experimental setup for time-integrated reflectivity measurements.

4.2: Time-integrated reflectivity versus intensity for metals ( $\mathrm{Al}$ and $\mathrm{Au}$ ), a semiconductor $(\mathrm{Si})$, and an insulator $\left(\mathrm{SiO}_{2}\right)$ with $\mathrm{S}$ - and $\mathrm{P}$-polarized light. 88

4.3: The measured cold target reflectivity for the $\mathrm{Al}, \mathrm{Au}, \mathrm{Si}$, and $\mathrm{SiO}_{2}$ targets for $\mathrm{S}$ and $\mathrm{P}$ polarization

4.4: The reflectivity of an Au target with $~ 3 \%$ ASE prepulse energy introduced. .90

4.5: Average ion charge state, normalized collision frequency at critical density, and normalized electron density versus temperature for $\mathrm{Al}$ and Au. 
4.6: The percentage linear inverse Bremsstrahlung absorption for Spolarized light and the percentage resonance absorption and total absorption $=$ inverse Bremsstrahlung + resonance absorption for $\mathrm{P}$ polarized light versus temperature for the $\mathrm{Al}$ and $\mathrm{Au}$ targets.

4.7: The reflected spectrum peak was Doppler-shifted to shorter wavelengths than the incident spectrum peak by hydrodynamic plasma motion.

4.8: Calculated plasma expansion velocity and normalized scale length versus temperature for the $\mathrm{Al}$ and $\mathrm{Au}$ targets.

4.9: Illustration of resonance absorption occurring from the resonant coupling of P-polarized light to an electrostatic plasma wave at the critical density.

4.10: Inferred temperature versus measured intensity for the $\mathrm{Al}$ target.

4.11: The coupling parameter versus temperature for $\mathrm{Al}$ and $\mathrm{Au}$. 106

4.12: The plasma coupling parameter as a function of density and temperature for $\mathrm{Al}$ and $\mathrm{Au}$.

4.13: Simulation results from the LASNEX code for Al reflectivity and peak electron temperature compared to the reflectivity data

4.14: The electron density, electron temperature, and pulse intensity are shown as functions of time.

4.15: Comparison of density shown in Figure 4.14 and density calculated without recombination shows insignificant recombination. 
4.16: Comparison of avalanche and tunneling ionization rates versus laser intensity.

4.17: This comparison shows that avalanche ionization is unimportant compared to photoionization for intensities as low as $10^{14} \mathrm{~W} / \mathrm{cm}^{2}$.

4.18: Total, polar, nonpolar TA, LA, and nonpolar TO electron-phonon scattering rates in $\mathrm{SiO}_{2}$ versus electron energy.

4.19: This comparison and the comparison given in Figure 4.19 (b) show that Eq. (4.25) is a high-intensity approximation for the electron temperature that breaks down near $10^{14} \mathrm{~W} / \mathrm{cm}^{2}$

4.20: The final electron temperature determined from Eq. (4.26) is compared to its numerical counterpart. This comparison shows Eq. (4.26) is reliable in the high-intensity regime.

4.21: This comparison shows Eq. (4.27) to be an accurate representation of the time-dependent density. 125

4.22: This comparison shows that Eq. (4.28) is a high-intensity approximation for eleçtron density. 126

4.23: Plot of Eq. (4.31), the relaxation time, as a function of the electron temperature. 128

4.24: A comparison of the equilibration and the tunneling ionization rate. 129

4.25: Calculated reflectivity versus intensity for the real Drude conductivity model is compared to reflectivity data in quartz $\left(\mathrm{SiO}_{2}\right)$. 131 
4.26: Measured reflectivity as a function of incident pulse intensity using a quartz target is compared to the single ionization and the multiple ionization, non-LTE model.

5.1: Experimental pump-probe setup for space- and time-resolved reflectivity measurements.

5.2: An imperfect target alignment displayed an oscillatory reflection signal.

5.3: Measured low-intensity reflectivity curves for $\mathrm{SiO}_{2}, \mathrm{Si}, \mathrm{Al}$, and $\mathrm{Au}$ for $\mathrm{S}$ and $\mathrm{P}$ polarization.

5.4: A linear interpolation of the low-intensity reflectivity counts from the calibration target is used to determine absolute reflectivity values.

5.5: The reflected and transmitted signal are spatially resolved over the plasma profile.

5.6: Reflectivity, transmission, and relative scatter from an $\mathrm{SiO}_{2}$ target irradiated with an S-polarized, $5 \times 10^{15} \mathrm{~W} / \mathrm{cm}^{2}$ pump pulse. Irises aperture in the transmitted and reflected image planes, so that only the central $-3 \mathrm{~mm}$ of the plasma is detected.

5.7: Reflectivity as a function of aperture size for a constant intensity $\left(\sim 10^{16} \mathrm{~W} / \mathrm{cm}^{2}\right)$. 143

5.8: The reflectivity response at the highest peak intensity measured $\left(\sim 10^{16} \mathrm{~W} / \mathrm{cm}^{2}\right)$ versus normalized intensity for $\mathrm{SiO}_{2}$ with S-polarization 
5.9: The incident and reflected intensity distributions and the reflectivity distribution assuming the plasma response shown in Figure $4.2(\mathrm{~g})$ for $\mathrm{SiO}_{2}$, S-polarization.

5.10: Reflectivity versus time in the central plasma region using an $\mathrm{SiO}_{2}$ target.

5.11: Temperature history simulation at pump intensities of $1016 \mathrm{~W} / \mathrm{cm}^{2}$, $10^{15} \mathrm{~W} / \mathrm{cm}^{2}, 10^{14} \mathrm{~W} / \mathrm{cm}^{2}$, and $10^{13} \mathrm{~W} / \mathrm{cm}^{2}$. 148

5.12: Peak electron temperature versus distance at the peak of the pulse, and at $0.22,0.54,1.70,4.42$ picoseconds after the peak.

5.13: Electron density normalized to critical density $\left(2.92 \times 10^{21} \mathrm{~cm}^{-3}\right)$ versus distance at the peak of the pulse, and at $0.22,0.54,1.70,4.42$ picoseconds after the peak.

5.14: Peak plasma temperature versus time with and without hydrodynamic expansion

5.15: Simulation of the plasma scalelength for $\mathrm{SiO}_{2}$ target at $1 \times 10^{16} \mathrm{~W} / \mathrm{cm}^{2}$. Scalength is calculated with and without the pondermotive force. Peak electron temperature with and without the pondermotive force is shown for reference.

5.16: Plasma absorption in the hydrodynamic scale length. 156

5.17: Reflectivity in the central plasma region versus time delay for $\mathrm{SiO}_{2}$ (fused quartz), $\mathrm{SiO}_{2}$ (glass), and lead glass. 
5.18: Comparison of the gradients in the peak electron temperature at the peak of the pump pulse for intensities of $10^{16} \mathrm{~W} / \mathrm{cm}^{2}$ and $10^{14} \mathrm{~W} / \mathrm{cm}^{2}$.

5.19: Reflectivity versus time delay in the central plasma region for intensities of $1 \times 10^{16} \mathrm{~W} / \mathrm{cm}^{2}, 6 \times 10^{15} \mathrm{~W} / \mathrm{cm}^{2}$, and $3 \times 10^{13} \mathrm{~W} / \mathrm{cm}^{2}$.

6.1: Temporal and spectral windowing on a chirped pulse.

6.2: The plasma mirror increases the contrast ratio by passing lowintensity prepulse and reflecting the high-intensity, femtosecond pulse.

6.3: Schematic experimental setup for spatial, temporal and reflective plasma mirror measurements.

6.4: Plasma mirror reflectivity versus intensity using uncoated $\mathrm{SiO}_{2}$. 175

6.5: Incident profile and reflected pulse profile at $1.4 \times 10^{15} \mathrm{~W} / \mathrm{cm}^{2}$, $2.8 \times 10^{14} \mathrm{~W} / \mathrm{cm}^{2}$, and $3.5 \times 10^{13} \mathrm{~W} / \mathrm{cm}^{2}$ 178

6.6: Timing between the oscillator and amplifier pulses. Induced prepulse pedestal and main pulse. Pulse with RG-630 saturable absorber in laser system. Main pulse with no saturable absorber, but reflected off the plasma mirror.

6.7: Schematic experimental setup for a high-dynamic-range autocorrelation measurement. 183

6.8: High-dynamic-range auto-correlation shows the plasma mirror prepulse suppression at Brewster's angle improves the contrast ratio by a factor $>625$. 183

6.9: Schematic diagram of the liquid plasma mirror. 185 
6.10: Liquid plasma mirror prepulse reduction with a $12 \%$ prepulse energy introduced and with a $12 \%$ prepulse energy and an RG-630 saturable absorber.

6.11: Calculated reflection coefficients versus time for a $100 \mathrm{fs}$ pulse with intensity $2.5 \times 10^{15} \mathrm{~W} / \mathrm{cm}^{2}$

6.12: Plasma mirror model results for the intensities $1.0 \times 10^{14} \mathrm{~W} / \mathrm{cm}^{2}$, $5.0 \times 10^{15} \mathrm{~W} / \mathrm{cm}^{2}, 7.5 \times 10^{15} \mathrm{~W} / \mathrm{cm}^{2}$, and $1 \times 10^{16} \mathrm{~W} / \mathrm{cm}^{2}$.

6.13: Absolute time of reflectivity rise versus intensity

6.14: A proposed double plasma mirror set-up for eliminating prepulse and postpulse from an intense, ultra-short laser pulse.

6.15: A proposed grating plasma mirror experiment for eliminating prepulse and postpulse from an intense, ultra-short laser pulse.

B1: $\alpha$ as a function of intensity for $T_{e}=1.4 \mathrm{eV}, 14 \mathrm{eV}, 79 \mathrm{eV}$, and $140 \mathrm{eV}$ 205 


\section{ABSTRACT}

Experiments were performed to characterize the creation and evolution of high-temperature $\left(T_{e} \sim 100 \mathrm{eV}\right)$, high-density $\left(n_{e}>10^{22} \mathrm{~cm}^{-3}\right)$ plasmas created with intense $\left(\sim 10^{12}-10^{16} \mathrm{~W} / \mathrm{cm}^{2}\right)$, ultra-short (130fs) laser pulses. The principle diagnostic was plasma reflectivity at optical wavelengths $(614 \mathrm{~nm})$. An array of target materials $\left(\mathrm{Al}, \mathrm{Au}, \mathrm{Si}, \mathrm{SiO}_{2}\right)$ with widely differing electronic properties tested plasma behavior over a large set of initial states. Time-integrated plasma reflectivity was measured as a function of laser intensity. Space- and timeresolved reflectivity, transmission and scatter were measured with a spatial resolution of $\sim 3 \mu \mathrm{m}$ and a temporal resolution of 130fs. An amplified, modelocked dye laser system was designed to produce $\sim 3.5 \mathrm{~mJ}, \sim 130 \mathrm{fs}$ laser pulses to create and nonintrusively probe the plasmas. Laser prepulse was carefully controlled to suppress preionization and give unambiguous, high-density plasma results.

In metals $(\mathrm{Al}$ and $\mathrm{Au})$, it is shown analytically that linear and nonlinear inverse Bremsstrahlung absorption, resonance absorption, and vacuum heating explain time-integrated reflectivity at intensities near $10^{16} \mathrm{~W} / \mathrm{cm}^{2}$. In the insulator, $\mathrm{SiO}_{2}$, a non-equilibrium plasma reflectivity model using tunneling ionization, Helmholtz equations, and Drude conductivity agrees with timeintegrated reflectivity measurements. Moreover, a comparison of ionization and Saha equilibration rates shows that plasma formed by intense, ultra-short pulses can exist with a transient, non-equilibrium distribution of ionization states. All targets are shown to approach a common reflectivity at intensities $\sim 1016 \mathrm{~W} / \mathrm{cm}^{2}$, indicating a material-independent state insensitive to atomic or solid-state details. 
Space- and time-resolved measurements reveal two reflectivity relaxation timescales in the central plasma region at $1016 \mathrm{~W} / \mathrm{cm}^{2}$. Comparison with laserplasma interaction simulations and nonlinear heat conduction theory associates the relaxation data with electron heat conduction and hydrodynamic expansion in the plasma.

Application of the plasmas to ultra-fast optical switching and prepulse suppression exhibited a femtosecond switching time with $70 \%$ efficiency. Prepulse suppression was improved by a factor $>625$. Spatial profile smoothing, spatial filtering, and $-10 \%$ pulsewidth reduction were observed in the reflected pulse. A regenerating, liquid target plasma switch demonstrated prepulse suppression feasibility for laser systems operating at up to $2 \mathrm{kHz}$. 


\section{CHAPTER 1:}

\section{INTRODUCTION}

Ultra-short pulse ( $<1 \mathrm{ps})$, high-intensity $\left(>10^{12} \mathrm{~W} / \mathrm{cm}^{2}\right)$ laser systems open new frontiers in the investigation of interactions between laser radiation and matter. A subpicosecond pulsewidth allows rapid, complex dynamics and energy transfer processes in optically excited atoms, molecules and solids to be frozen in time [1]. One of the most exciting new fields, however, arises when the ultra-short pulse energy is amplified - the creation of high-density plasmas by focusing amplified, ultra-short pulses to intensities which ionize atoms.

The goal of these studies is a quantitative understanding of the creation and evolution of these newly accessible, high-density plasmas as inferred by plasma reflectivity measurements. Analysis of material breakdown mechanisms, ionization physics, plasma density and temperature dynamics, linear and nonlinear plasma heating, high-intensity phenomena, electron heat conduction, and plasma hydrodynamics is included. The plasmas are characterized by femtosecond, nonequilibrium, ionization and extreme heating of a solid before picosecond relaxation from hydrodynamic expansion and ionic recombination can occur. Plasma creation is studied by space- and time-integrated, self-reflectivity measurements using high-intensity, ultra-short optical pulses. Subsequent plasma evolution and relaxation is studied by space- and time-resolved, pumpprobe reflectivity, transmission and scatter measurements.

From time-integrated data, using an $\mathrm{SiO}_{2}$ target, ionization rates are calculated based on a non-equilibrium, rate equation ionization model. Conductivity is also calculated for the transition from solid to plasma using the ionization model and a Drude model [2]. Ionization and conductivity 
calculations are integrated into a single model which gives reflectivity by using the Helmholtz wave equations for electromagnetic field propagation. The calculated reflectivity compares directly with time-integrated reflectivity measurements.

From space and time-resolved reflectivity data, two distinct plasma processes, nonlinear electron heat conduction and hydrodynamic expansion, are identified. Electron conduction and hydrodynamic timescales are inferred based on a comparison between hydrodynamic plasma simulations and space- and time-resolved reflectivity data. Temperature and density simulations for evolving plasmas are also produced. The plasma simulation results include wave propagation physics allowing a direct comparison with time-resolved reflectivity data.

The sum of these measurements and simulations by no means gives a complete understanding of dense plasmas. However, the work detailed in this dissertation is a significant step in the detailed characterization and understanding of this state of matter. We believe the time-integrated results are the first detailed set of measurements of high-density plasmas at intensities up to $\sim 10^{16} \mathrm{~W} / \mathrm{cm}^{2}$ over the entire range of material classes (insulator, semiconductor, and metal). Moreover, at the highest measured intensities, the first observation of a material-independent plasma reflectivity state is found. The space- and time-resolved measurements include the first direct identification of a transition between electron conduction and hydrodynamic cooling physics in a dense plasma. Finally, an additional goal of this research was to apply the insight gained from reflectivity measurements to a proposed plasma-based device for prepulse suppression in intense, ultra-short pulse laser systems, or for ultra-fast optical switching. The first direct measurement of prepulse suppression with an ultra-fast optical plasma switch is presented. 
What is so special about plasmas created by ultra-short pulses? Although lasers have been used to create plasmas since the early 1960 's, ultra-short pulse plasmas are in an entirely new density-temperature regime. The electron density and temperature of such plasmas is near or above solid density and can approach kiloelectron volts, respectively (Figure 1.1). The conditions of this laboratory plasma approaches that of stellar plasmas (Figure 1.1). These plasmas are matter under extreme conditions not found terrestrially. The extreme state is not attainable with longer laser pulses or by other easily controlled, i.e. nonthermonuclear, methods.

This unique state of matter is essentially a solid that has been superheated without significant expansion [3, 4]. This transient state exists long enough $(\sim 1 p s)$ that it can be probed with another ultra-short pulse to extend condensed matter measurements to temperature-density regimes inaccessible by other methods. For example, Milchberg et. al. [3] demonstrated that the resistivity of a material (Al) could be measured over a temperature range greater than four orders of magnitude without significant expansion. Conventional solid state measurements approach only $\sim 1 \%$ of this temperature range. Another example of unique femtosecond excitation, is the work of Reitze et. al. [4]. Liquid carbon optical properties were studied by femtosecond melting of graphite and diamond samples. Solid carbon has a melting temperature $\sim 5000 \mathrm{~K}$ (far above that of any containment vessel) and sublimes at low ambient pressure, so longterm confinement at elevated temperature and pressure is impossible. Thus, measurements depend on transient melting and probing. Previous methods using pulsed ohmic heating, shock waves, high-pressure cells and long pulse laser heating could not create a confined temperature-density state or probe with adequate time resolution to see liquid carbon's metallic character. Reitze's measurement succeeded because intense, ultra-short pulses were able to both 
create a high-temperature, high-pressure carbon state and probe this state in a critical time window ( $1 \mathrm{ps})$ of confinement before it vanished by hydrodynamic surface expansion. The measurements presented here partially extend Reitze's analysis to a higher-intensity regime where the ionization is such that the state goes beyond melting to an initially confined plasma state.

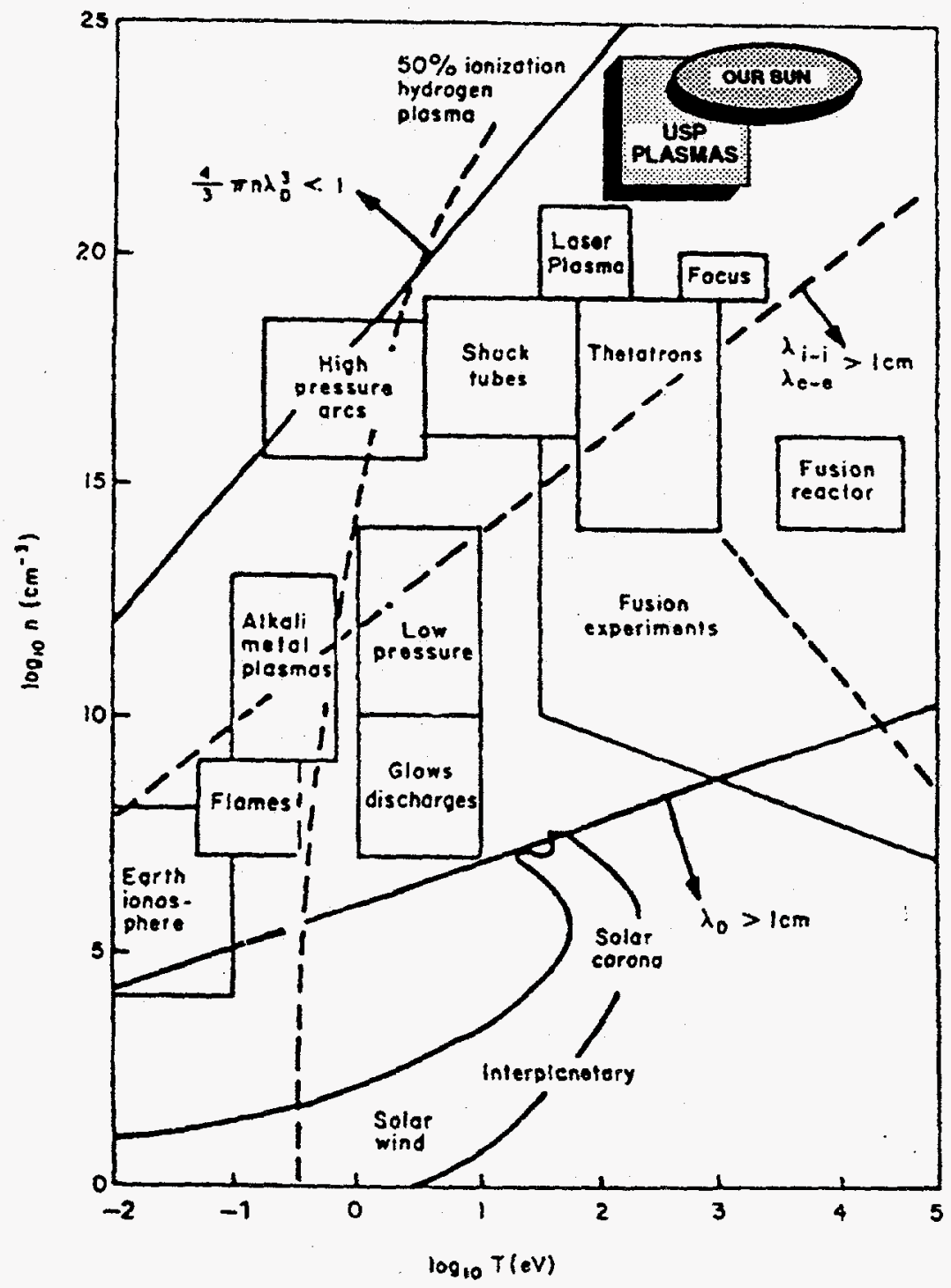

Figure 1.1: Ultra-short pulse (USP) lasers form the highest temperaturedensity laboratory plasmas and approach stellar interior states (after [5]). 
Beyond the new "solid state" aspects, ultra-short pulse plasmas are also special because the initial plasma temperature and density gradients are extremely, steep. Steep gradients cause rapid relaxation with the release of, among other things, a bright, picosecond burst of $x$-rays [6]. Short pulses of $x-$ rays can be applied to many systems or experiments that demand high spatial and temporal resolution [7].

Finally, the plasma formation is as interesting as its relaxation. Conventional laser plasmas absorb a large fraction of the laser field energy and ionize slowly. Electrons are heated by inverse Bremsstrahlung to energies that collisionally create a plasma. Ultra-short pulses, however, have peak fields approaching or exceeding atomic binding energies so the laser field acts mainly as an electron extractor. The field directly can ionize atoms without significant heating compared to the ionization state. This collisionless ionization is known as multi-photon and tunneling ionization [8-10]. Highly ionized, yet relatively cool, plasmas are currently being studied as a possible scheme for photopumped, recombination $x$-ray lasers $[11,12]$.

This dissertation begins by introducing the laser technology (Chapter 2) and the physical plasma conditions (Chapter 3). The laser system and plasma characteristics are very different from those commonly encountered in laserplasma experiments. Therefore, it is valuable to put both the experiment and the plasma in perspective.

In Chapter 4 we investigate the creation of plasmas as a function of intensity and the initial, cold target, electronic conditions. The time-integrated reflectivities from metals ( $\mathrm{Al}$ and $\mathrm{Au}$ ), a semiconductor ( $\mathrm{Si}$ ), and an insulator $\left(\mathrm{SiO}_{2}\right)$ are measured. Specific absorption mechanisms are discussed and analytically compared to reflectivity data on metals. We then focus on the plasma initiation out of local thermodynamic equilibrium (LTE) using self- 
reflection data from the insulator $\left(\mathrm{SiO}_{2}\right)$ target and a plasma model. It was recognized by Murnane [13] that intense, ultra-short pulse lasers may produce plasmas out of LTE, because the models relying on LTE distributions may not be reliable during the formation process. This is in contrast to longer pulse ( $>1 \mathrm{ps})$ interactions in which the electron distribution is approximately Maxwellian, and ions have time to collisionally equilibrate their ionization states to a Saha distribution during the interaction with the pulse

Inverse Bremsstrahlung heating followed by collisional ionization is the dominant mechanism for breakdown in optical glasses by irradiation with lower intensity and longer pulse lasers $[14,15]$. In Chapter 4 , we show that photoionization by electron tunneling [10] becomes the dominant breakdown mechanism as the laser intensity is increased and the pulsewidth is decreased. This transition was anticipated, but not investigated in detail, by Bloembergen [14], In ultra-short pulse, high-intensity laser interactions, the transition occurs because the photo-ionization rate is much larger than the inverse Bremsstrahlung rate which controls the temperature and, consequently, determines the collisional ionization rate.

Because of the transition in the ionization mechanism with increasing laser intensity, the state in which a plasma is formed switches from a LTE to a NLTE state. At lower intensities, the electron production is temperature-driven and occurs in LTE due to the plasma's high collisionality. However, at higher intensities, the plasma is formed in a NLTE state, because the photo-ionization rate is greater than the rate to relax to a state of LTE. In addition, the rapid photo-ionization produces a lower temperature plasma for a given degree of ionization than predicted by LTE theory. In fact, the minimal heating motivates schemes for recombination $x$-ray lasers in field-ionized gaseous plasmas $[4,11$, $16]$, as previously mentioned. Once the pulse is gone, plasma evolution becomes 
temperature-driven and the transient NLTE state collisionally relaxes to a LTE state.

Chapter 5 describes the evolution of the dense plasma using temporallyand spatially-resolved reflectivity data and a sophisticated plasma simulation. Following excitation, the heated plasma relaxes in two ways: prompt nonlinear heat conduction from electrons, followed about 1ps later by significant, but much slower, hydrodynamic expansion. Simulations indicate that two distinct reflectivity relaxation timescales found in the data verify both of the plasma relaxation mechanisms.

Chapter 6 presents the first direct measurement of prepulse suppression using a plasma-based, ultra-fast optical shutter. The plasmas described in Chapters 4 and 5 are put into practice to improve the contrast ratio of highintensity laser systems. The resulting pulse's spatial and temporal characteristics are measured. The technique is shown to also apply to high-repetition-rate $(<2 \mathrm{kHz})$ laser systems by replacing the solid target with a rapidly regenerating liquid target.

Chapter 7 summarizes the conclusions drawn in this work. 


\section{CHAPTER 2:}

\section{THE AMPLIFIED, ULTRA-SHORT PULSE LASER SYSTEM}

\subsection{INTRODUCTION}

An important part of this research involved the design, construction, and operation of an amplified, mode-locked dye laser [17]. When this research began, the laser system represented the state-of-the-art in high-power, ultra-short pulse laser design.

Experimental results from the interaction of high-intensity, ultra-short laser pulses with matter can depend heavily on the specific laser technology used. Many laser systems will produce high-energy, short pulses. Only "clean", ultra-short ( 100fs) laser pulses, however, can produce high-density plasmas and unambiguously distinguish their unique properties from artifacts of longer, noisier laser pulses. A clean pulse has an exceptionally high contrast between the main femtosecond pulse energy and a much longer ( $\sim \mathrm{ps}-\mathrm{ns})$ energy pedestal before and after the main pulse. The source of this energy pedestal is amplified spontaneous emission (ASE) in laser amplifiers or incomplete pulse compression in chirped pulse amplification (CPA) [18] laser systems. Because of this sensitivity to laser pulse quality, a detailed description of the laser system used in these experiments is presented in this chapter.

The common use of excimer amplifiers is a good example of how some lasers can complicate the success and interpretation of high-density plasma experiments [6]. The large excimer amplifier small-signal gain generates copious amounts of ASE [19] which creates a significant energy pedestal for nanoseconds before and after the main ultra-short pulse. To study high-density, hightemperature laser plasmas, it is critical that the laser pulse have steep rising and 
falling edges with an absolute minimum of prepulse and postpulse energy. Ideally, the maximum energy is delivered in the minimum time. This allows the majority of the energy to be absorbed in the target's skin depth before plasma expansion, which results in lower plasma densities and temperatures. The skin depth, $\delta$, is the distance where the electromagnetic wave damps to $1 / e$ of its amplitude

$$
\delta=\frac{c}{\sqrt{2 \pi \omega \sigma}}
$$

where $\sigma$ is the plasma conductivity, $c$ is the speed of light and $\omega$ is the pulse vacuum frequency. Any significant deviation from the ideal clean pulse may change results in an unknown and nonlinear manner. Results are altered because prepulse energy absorption above the damage fluence causes a lowdensity $\left(<10^{21} \mathrm{~cm}^{-3}\right)$, low-temperature $(-10 \mathrm{eV})$ preformed plasma to form at the target surface. Preformed plasma absorbs and scatters the main pulse energy and changes the hydrodynamic plasma expansion. An excimer system also produces a poor beam quality, because the laser frequency must be doubled in a nonlinear medium to produce a UV $(-308 \mathrm{~nm})$ input near the excimer amplifier gain curve maximum [19]. Moreover, excimer amplifiers contain a non-uniform plasma discharge in the excimer gas, so the output beam profile is equally nonuniform. For high-intensity studies, it is essential that the spatial beam quality be sufficient for near diffraction-limited focusing. Finally, because an excimer amplifier operates in the UV spectral region, where group velocity dispersion (GVD) is high, the pulse broadens significantly while propagating through optical components. High-intensity experiments demand as short a pulse as possible, so GVD must be minimized. 


\subsection{THE EVOLUTION OF AMPLIFIED, ULTRA-SHORT PULSE LASERS}

Since the laser's invention, one goal of physicists has been to obtain higher powers and shorter pulsewidths that open new frontiers in the investigation of physical processes. These processes occur only on extremely short timescales or in the presence of very high energy density. Figure 2.1 illustrates how laser advances drive scientific discovery, particularly in the area of radiation interaction with plasma and atomic systems.
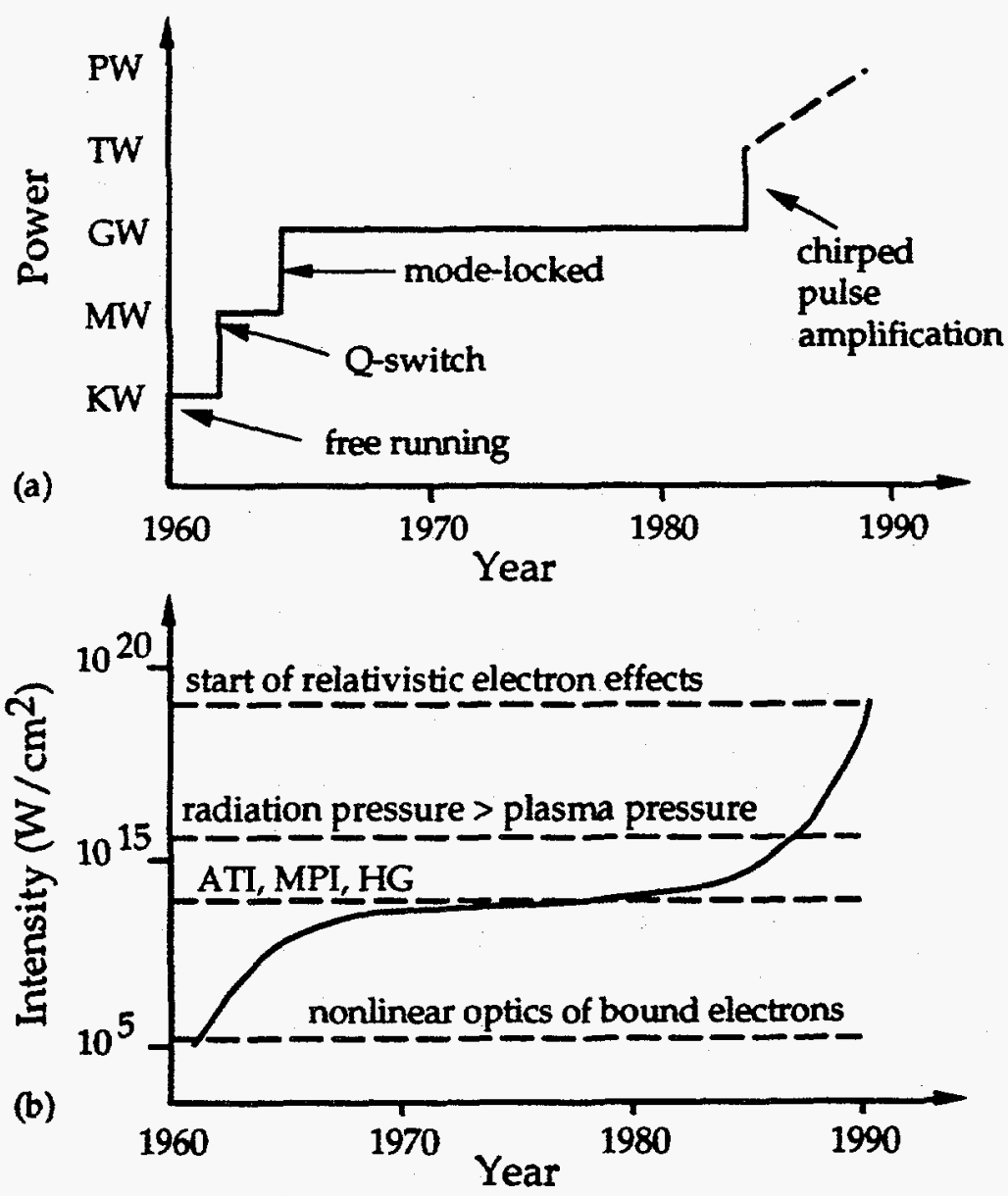

Figure 2.1: (a) New laser technology drives progress to intensity thresholds where (b) new physical phenomena occur (after [20]).

In the early 1960 's, Q-switching and cavity dumping produced the first significant advance toward short pulses with high peak power - nanosecond, 
megawatt laser pulses focusable to intensities $>10^{5} \mathrm{~W} / \mathrm{cm}^{2}$ (fields $>8.7 \times 10^{5} \mathrm{~V} / \mathrm{m}$ ). Laser fields at these intensities significantly perturb the atomic field (1 atomic unit $=5.14 \times 10^{11} \mathrm{~V} / \mathrm{m}$ ) allowing nonlinear optical interactions with bound electrons to occur.

Q-switching is a technique where the laser resonator quality factor (the cavity " $Q$ ") is initially kept low, so lasing will not occur. The cavity $Q$ is $2 \pi$ (energy stored in the resonator/energy lost in one cycle). Meanwhile, a large population inversion is produced by strong laser medium pumping. The cavity $Q$ is then switched to a high value. Lasing occurs with a very large population inversion, i.e., a large gain. The pulse is ejected with a width on the order of the cavity decay time $(\sim \mathrm{ns})$ [21]. The cavity $Q$ is switched by an intracavity rotating mirror, saturable absorber, acousto-optic or electro-optic modulator. In this research, a Q-switched, neodymium-doped yttrium aluminum garnet (Nd:YAG) laser was used to pump dye amplifiers and produce millijoule, femtosecond pulses.

Cavity dumping is essentially Q-switching a cavity without building up extra population inversion. Intracavity energy is directed out of the cavity by an acousto-optic or electro-optic modulator. Typically, continuous wave (cw) lasers emit about $4 \%$ of their intracavity energy through the output coupler. This means that cavity-dumped lasers produce pulses with about 25 times the $\mathrm{cw}$ energy in approximately the time it takes to empty the cavity of light $(\sim \mathrm{ns})$.

The first demonstration of mode locking in 1965 [22] produced pulses with widths in the "ultra-short" picosecond regime. This marked a reduction to pulsewidths orders of magnitude shorter than previously attained nanosecond pulses. In the following years, a number of mode-locked laser designs were developed based upon active and passive mode locking of flashlamp pumped dye lasers [23]. 
The first cw-pumped, mode-locked laser was reported by Ippen [24] in 1972. The laser produced 1.5ps pulses using a linear, or standing wave, cavity geometry. In 1981 Fork [25] used a ring cavity geometry and a technique termed "colliding pulse mode locking," or CPM, to bring laser pulses into the $100 \mathrm{fs}$ regime. In a ring cavity, pulses travel in both directions around a ring resonator, rather than acting as a standing wave. In a CPM laser, the gain medium and the saturable absorber are separated by one-quarter of the round-trip path length. A saturable absorber is a material that absorbs heavily at low intensities, but saturates at high intensities to become nearly lossless. It allows the CPM laser to produce ultra-short pulses by the mode locking technique described in Section 2.4. Two counterpropagating pulses collide in the saturable absorber and are at their maximum separation (one-half the round-trip distance) when one pulse enters the gain medium. This geometry allows deeper absorber saturation as well as maximum and equal gain for the pulses in the gain medium, so pulses are short, energetic and stable. A deep saturation of both the saturable absorber and gain media also produces a large, bandwidth broadening, self-phase modulation (SPM) in the pulse [26-30]. SPM is discussed in detail in Section 2.5.1.2. Pulses suffer a significant amount of pulse broadening, however, because group velocity dispersion (GVD) over the large bandwidth occurs in the mirrors, saturable absorber and ring resonator gain medium. In 1985, Valdmanis [31] included a set of four prisms in the laser cavity that allowed group velocity dispersion to be continuously adjusted through both positive and negative values. Valdmanis was able to demonstrate the shortest dye laser oscillator pulses of the time, 27fs, by balancing increased bandwidth from SPM with pulse compressing effects of saturable absorption, saturable gain, and GVD compensation [31]. The CPM dye laser with dispersion compensation provided a method of producing stable, $\sim 100 \mathrm{fs}$ pulses which was state-of-the-art when this 
research began. Since that time, however, laser technology has advanced through the use of new broadband solid-state laser materials, new mode locking techniques and high order dispersion compensation. These advances have produced a record $8.3 \mathrm{fs}$ pulses [32] directly from an oscillator by careful control of both low and high order intracavity dispersion.

To produce pulses shorter than about $27 \mathrm{fs}$, one can see from Figure 2.2 that the bandwidth requirement goes up dramatically. Since dyes are limited to bandwidths near $13 \mathrm{~nm}$ (using $\lambda=620 \mathrm{~nm}$, 100fs FWHM), addition of bandwidth and subsequent pulse compression must occur. Adding bandwidth with large amounts of SPM and recompressing is difficult intracavity beyond the level of the CPM laser. In 1982, Shank [33] reported 30fs pulses using an extra-cavity technique to compress ultra-short pulses. Extra-cavity compression was accomplished by focusing ultra-short pulses into an optical fiber. High peak pulse power induced SPM that increased pulse bandwidth and added a timevarying instantaneous frequency, or chirp, to the pulse. Fork [34] demonstrated that bandwidth could be increased beyond the visible spectrum. Diffraction grating [35] and prism [36, 37] techniques were then used to compress linearly chirped bandwidth additions and produce a near transform-limited pulse. Shank's technique $[33,34,38-41]$ eventually led to the shortest optical pulses ever produced, only $6 \mathrm{fs}$ in duration [38].

Amplification of mode-locked dye lasers to intensities up to $\sim 1016 \mathrm{~W} / \mathrm{cm}^{2}$ allowed, for the first time, interactions in which the peak laser field $\left(-2.75 \times 10^{11} \mathrm{~V} / \mathrm{m}\right)$ was on the order of the atomic field. Effects $[8,9]$ such as multiphoton ionization (MPI) and low order harmonic generation (HG) were enhanced. New effects $[8,9]$ such as above threshold ionization (ATI) and high order harmonic generation in neutral atoms were observed as shown in Figure 2.1. 


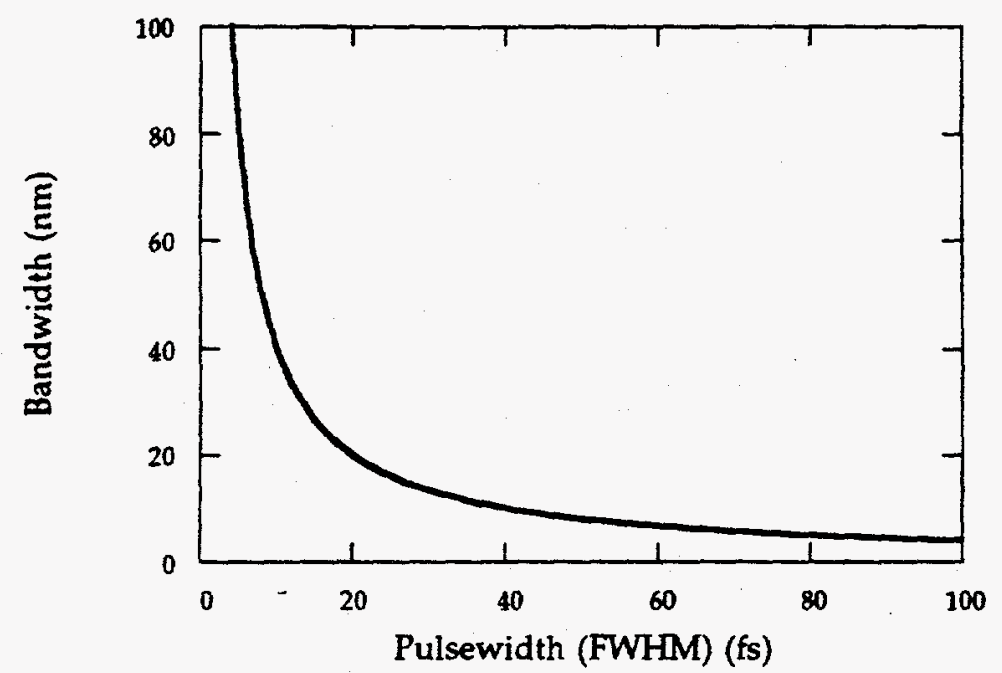

Figure 2.2: Bandwidth requirement for a transform-limited, 614nm, Gaussian pulse as a function of pulsewidth.

The energy of amplified dye and solid-state laser systems remained at several millijoules until demonstration of a powerful new amplification technique - chirped-pulse amplification (CPA) [18]. Using CPA, ultra-short pulse powers have reached terrawatts $\left(10^{12} \mathrm{~W}\right)$ and are rapidly approaching petawatts $\left(10^{15} \mathrm{~W}\right)$. At one terrawatt, focused intensities $>10^{18} \mathrm{~W} / \mathrm{cm}^{2}$ can be achieved. These intensities have a corresponding field strength of approximately $2.75 \times 10^{12} \mathrm{~V} / \mathrm{m}$. This field strength is well above the atomic potential, so interactions between the laser field and unbound electrons are highly nonlinear and become relativistic $[20,42]$.

CPA systems using new broadband solid-state materials exhibit all the necessary properties for amplification to energies beyond several millijoules. Broadband solid-state materials support amplification over the entire ultra-short optical pulse bandwidth. A pulse amplifier must have a gain bandwidth which satisfies the uncertainty principle relation, $\Delta v \Delta \tau \sim 0.5$, where $\Delta v$ is the amplifier bandwidth and $\Delta \tau$ is the pulsewidth. An amplifier must, for example, have at 
least $16 \AA$ of bandwidth to amplify a $1 \mu \mathrm{m}$ picosecond pulse. However, the amplifier gain profile is not flat over the pulse spectrum. This leads to gain narrowing in the amplifier and, consequently, pulse broadening. Therefore, amplifiers usually need a bandwidth of several hundred angstroms to support an ultra-short pulse [20].

New solid-state amplifier materials also allow much more energy storage for greater amplification. For efficient energy extraction, the input fluence should be near the amplifier saturation fluence

$$
F_{\text {sat }}=h v / 2 \sigma
$$

where $h$ is Planck's constant and $\sigma$ is the stimulated emission cross section. Dyes and excimers have a large stimulated emission cross section and a saturation fluence of about $1 \mathrm{~mJ} / \mathrm{cm}^{2}$. An amplifier with an amplifying bore of several square centimeters ( 1 inch diameter), a practical laboratory size, can therefore generate only a few millijoules of energy. Solid-state amplifiers such as Nd:Glass, Ti:Sapphire, and Alexandrite have a saturation fluence of about $1 \mathrm{~J} / \mathrm{cm}^{2}$ allowing for extraction of energy several orders of magnitude higher.

Finally, and most importantly, CPA keeps intensity low enough to avoid nonlinear wavefront distortion in amplifiers. Without CPA, broad amplifier bandwidth and high energy storage would be useless. Laser wavefront distortion would render the beam nearly unfocusable and could result in damage to the laser system by self-focusing. Wavefront distortion and self-focusing occur because an intensity-dependent index of refraction causes spatial inhomogeneities in the beam to grow. They grow at an exponential rate, $g$, equal to [43]

$$
g=\left(k / n_{0}\right)(\lambda / 2 \pi L) B
$$


for $k<300 \mathrm{~cm}^{-1}$, where $k$ is the spatial wave number, $\lambda$ is the wavelength, $n_{0}$ is the index of refraction and $L$ is the amplifying medium length. $B$ refers to the number of accumulated waves of nonlinear phase shift

$$
B=\frac{2 \pi}{\lambda n_{0}} \int_{0}^{L} n_{2} I(z) d z
$$

where $n_{2}$ is the nonlinear index of refraction, $I$ is the intensity and $z$ is the distance along the amplifier.

CPA minimizes $B$ by spreading energy out over time during amplification. The oscillator pulse ( 100fs) is first stretched in time by at least three orders of magnitude, then amplified in broadband solid-state materials by a factor $\sim 10^{10}$, then recompressed using the reverse process of stretching (pulse compression). Stretching and compression are accomplished by negatively and positively dispersive elements such as a grating pair [35]. In this procedure, both high energy storage and broad amplifying bandwidth can be used without nonlinear distortions degrading the pulse, because input fluence is kept constant while intensity is reduced. CPA systems [20, 44-47] producing $~ 0.5-10], 100 \mathrm{fs}-1 \mathrm{ps}$ (multiterrawatt) pulses, which can be focused to an intensity approaching $10^{19} \mathrm{~W} / \mathrm{cm}^{2}$, represent the state-of-the-art at the time of this writing. Future systems capable of intensities exceeding $10^{21} \mathrm{~W} / \mathrm{cm}^{2}$ are envisioned at several facilities around the world.

\subsection{APPLICATIONS OF ULTRA-SHORT PULSE LASERS}

A tool that acts on a $100 \mathrm{fs}$ timescale is useful in a wide array of applications other than plasma physics. Examples include nearly every scientific discipline that studies ultra-fast dynamic processes. Ultra-short pulses have been used in studying solid-state interactions of electron-hole systems [48], lattices [49], and excitons [50] in semiconductors. In electronics, femtosecond pulses have been used to characterize short electrical pulses from photoconductive 
switches [51]. Chemists have used ultra-short pulses to study subpicosecond photoisomerization in organic molecules during chemical reactions [52]. Biologists have measured timescales for photodissociation in proteins using ultra-fast lasers [53].

When ultra-short pulses are amplified to high intensities (1012. $1016 \mathrm{~W} / \mathrm{cm}^{2}$ ), applications generally narrow to studies of plasma, atomic, and fundamental particle systems, because all materials are vaporized at these intensities. New, hot $\left(T_{e}>100 \mathrm{eV}-\mathrm{keV}\right)$, dense $\left(n_{e}>10^{22} \mathrm{~cm}^{-3}\right)$ plasmas are being created (in the laboratory!) which resemble a star's interior environment. These plasmas have steep temperature and density gradients, so ultra-fast bursts of $x$ rays are produced as the plasma rapidly relaxes [13]. The x-rays may eventually be applied to time-resolved studies with subpicosecond resolution, photolithography with $x$-ray resolution, or a number of other areas [7].

Finally, when ultra-short pulses are amplified to ultra-high intensities $\left(>10^{18} \mathrm{~W} / \mathrm{cm}^{2}\right)$, laser-electron interactions becomes highly nonlinear and relativistic [42]. New phenomena with applications ranging from advanced particle accelerators to ultra-short wavelength radiation sources are predicted. These phenomena include [20, 42]: (1) laser excitation of large-amplitude plasma waves (wake fields), (2) relativistic optical guiding of laser pulses by plasmas, (3) optical guiding by preformed plasma channels, (4) laser frequency amplification by ionization fronts or plasma waves, (5) relativistic harmonic generation, (6) stimulated backscattering from plasmas and electron beams, (7) nonlinear Thompson scattering from plasmas and electron beams, and (8) cooling of electron beams by intense lasers. 


\subsection{MODE LOCKING LASERS}

\subsubsection{Importance to High-Intensity Systems}

Mode locking is fundamental to achieving ultra-short pulse, high-intensity laser-matter interactions. All currently available ultra-short pulse, high-intensity laser systems rely on mode locking, in one form or another, to achieve pulses short enough for the high peak intensities used in photo-ionization and highdensity plasma experiments. Moreover, mode locking allows time-resolved experiments with unparalleled, subpicosecond, time resolution and allows access to states that exist only in an extremely narrow window of time. For example, the optically measurable metallic liquid phase of carbon [4] is known to exist in a laboratory environment for only about 1ps. Because mode locking is such a powerful tool in current laser research and is critical to the work presented here, it is explained in the following sections.

\subsubsection{Mode Locking Basics}

Longitudinal cavity modes in most lasers have random phase relations with uncorrelated variations in time. The total intensity is the sum of the individual mode intensities. In mode-locked lasers, however, the phase of each lasing longitudinal mode is forced to correlate to the phase of all other modes. The resulting intensity is not simply the sum of the individual mode intensities. Instead, individual pulses have an intensity equal to (the number of phase-locked lasing longitudinal modes) $x$ (the sum of the individual mode intensities). This relation is the key to obtaining strong electromagnetic fields for high-intensity interactions and is explained in detail below.

Consider the $m$ th longitudinal mode electric field with frequency $\omega_{m}$, wavenumber $k_{m}$, amplitude $\varepsilon_{m}$, polarization $\hat{\varepsilon}_{m}$, and phase $\phi_{m}$ in a laser cavity of length $L$, 


$$
\begin{aligned}
\mathrm{E}_{m}(z, t) & =\hat{\varepsilon}_{m} \varepsilon_{m}(z) \sin \left(\omega_{m} t+\phi_{m}\right) \\
& =\hat{\varepsilon}_{m} \varepsilon_{m} \sin k_{m} z \sin \left(\omega_{m} t+\phi_{m}\right)
\end{aligned}
$$

where

$$
k_{m}=m \frac{\pi}{L}, m=1,2,3, \ldots
$$

and

$$
\omega_{m}=k_{m} c=m \frac{\pi c}{L}, m=1,2,3, \ldots
$$

To simplify, we use scalar quantities by assuming all mode fields are the same magnitude, $\varepsilon_{0}$, and polarization. Also, consider the simplest case of phaselocking where all modes have a phase angle of zero. The total electric field in the cavity is then

$$
E(z, t)=\sum_{m} E_{m}(z, t)=\varepsilon_{0} \sum_{m} \sin k_{m} z \sin \omega_{m} t
$$

There are typically millions of modes oscillating in a laser cavity so we can rewrite $k_{m}$ and $\omega_{m}$ in Eq. (2.5) as

$$
\begin{aligned}
& k_{m}=(M+n) \pi / L \\
& \omega_{m}=(M+n) \pi c / L
\end{aligned}
$$

where $M \sim 10^{6}$ and $n$ runs from $-\frac{1}{2}(N-1)$ to $+\frac{1}{2}(N-1)$, corresponding to a total of $N(\ll<)$ phase-locked modes centered at the frequency $M \pi x / L$. Then Eq. (2.6) becomes

$$
\begin{aligned}
E(z, t) & =\varepsilon_{0} \sum_{-(N-1) / 2}^{(N-1) / 2} \sin \frac{(M+n) \pi z}{L} \sin \frac{(M+n) \pi c t}{L} \\
& =\frac{1}{2} \varepsilon_{0} \sum_{n}\left(\cos \frac{(M+n) \pi(z-c t)}{L}-\cos \frac{(M+n) \pi(z+c t)}{L}\right)
\end{aligned}
$$

for the total electric field in the laser cavity. It can be shown [16] that Eq. (2.8) can be rewritten as

$$
E(z, t)=\frac{\varepsilon_{0}}{2}\left(\cos k_{0}(z-c t) \frac{\sin N \pi(z-c t) / 2 L}{\sin \pi(z-c t) / 2 L}-\cos k_{0}(z+c t) \frac{\sin N \pi(z+c t) / 2 L}{\sin \pi(z+c t) / 2 L}\right)
$$

where 


$$
k_{0}=\pi M / L
$$

A graphical representation of this sum of the electric fields, shown in Figure 2.3, illustrates that with phase-locked modes, a series of pulses of peak amplitude $N \varepsilon_{0} / 2$ occur at time intervals $2 L / c$ with a duration $2 L / c N$.

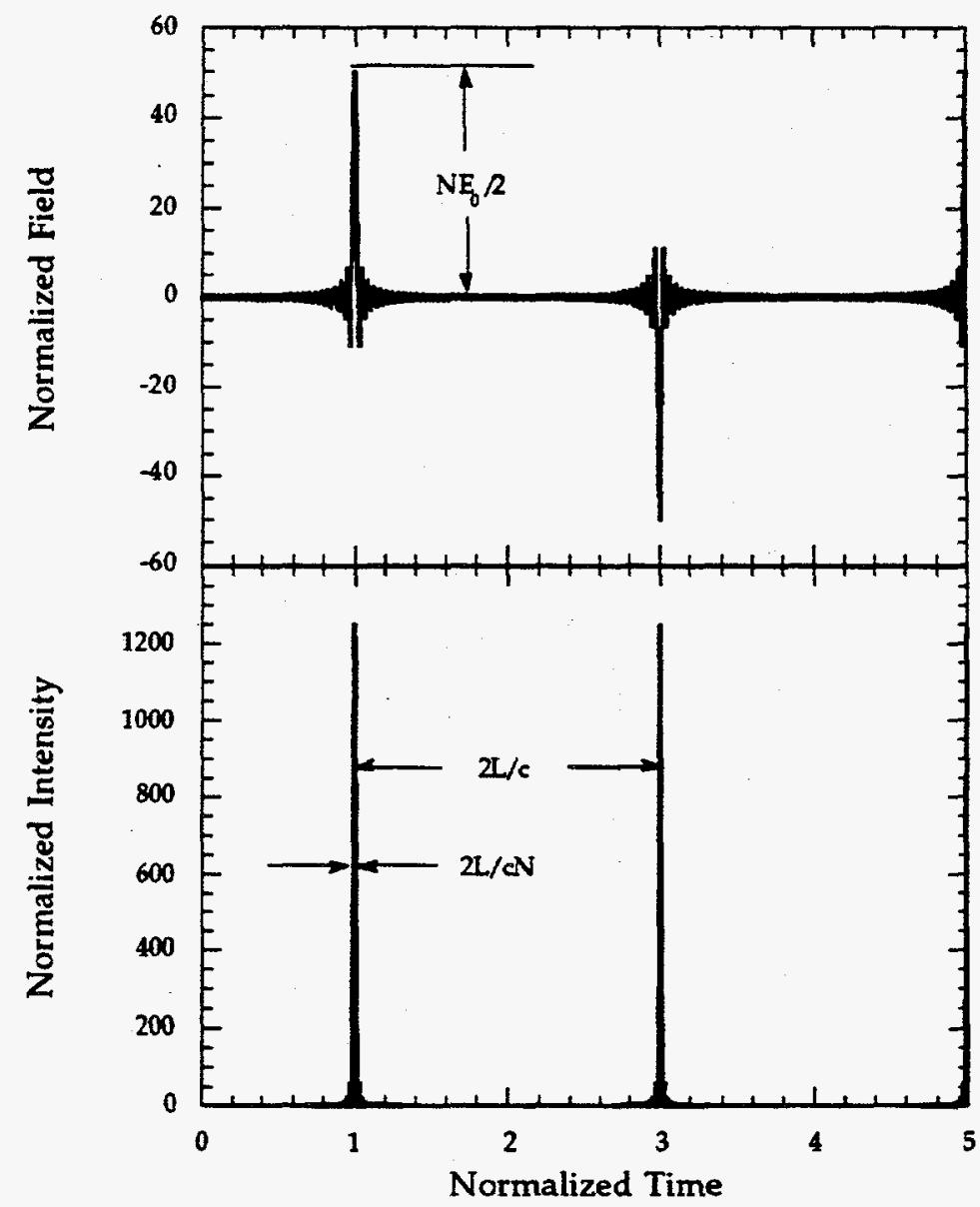

Figure 2.3: The field and intensity of a mode-locked laser with $N=100$ modes phase-locked. Field is in units of $\varepsilon_{0}$, length is in units of $L$ and time is in units of $L / C$.

The shortest pulse duration achievable with mode locking depends on the gain medium linewidth, $\Delta v_{g}$, and the longitudinal modes' frequency separation, $c / 2 L$. Under strong pumping conditions approximately 


$$
N=\frac{\Delta v_{g}}{c / 2 L}=\frac{2 L}{c} \Delta v_{g}
$$

longitudinal modes can oscillate simultaneously. Using the pulse duration found above, $\tau=2 L / c N$, one finds the shortest pulse length is approximately related to the inverse of the gain linewidth

$$
\tau_{\min }=\frac{2 L}{c N}=\frac{1}{\Delta v_{g}}
$$

The shortest obtainable pulsewidths from mode locking various common laser media are shown in Table 2.1. This shows that only liquid dyes and the more recently discovered broadband solid-state materials, such as Ti:Sapphire, have the exceptionally broad gain linewidth necessary to support ultra-short (subpicosecond) pulse formation.

\begin{tabular}{ccc}
\hline Medium & Gain Linewidth $\left.\mathbf{s}^{-1}\right)$ & Min. Pulsewidth (s) \\
\hline HeNe & $1.5 \times 10^{9}$ & $6.7 \times 10^{-10}$ \\
Ruby & $6.0 \times 10^{10}$ & $1.7 \times 10^{-11}$ \\
Nd:YAG & $1.2 \times 10^{10}$ & $8.3 \times 10^{-11}$ \\
Dye (R-6G) & $6.0 \times 10^{13}$ & $1.6 \times 10^{-14}$ \\
Ti:Sapphire & $8.7 \times 10^{13}$ & $1.2 \times 10^{-14}$ \\
\hline \hline
\end{tabular}

Table 2.1: Pulsewidths achieoable by mode locking in various laser media.

In practice, mode locking can be divided into $\mathrm{AM}$ mode locking, where the field amplitude is modulated and FM mode locking, where the field phase is modulated. 


\subsubsection{Active AM Mode Locking}

AM mode locking introduces a periodic gain or loss to the laser cavity by the addition of a device that locks the oscillating modes in phase. To see how this works, consider the electric field of mode $m$ in the cavity

$$
E_{m}(z, t)=\varepsilon_{m} \sin \left(\omega_{m} t+\phi_{m}\right) \sin k_{m} z
$$

If the amplitude $\varepsilon_{m}$ is modulated at frequency $\Omega$ with strength $\varepsilon$, then the field in Eq. (2.12) becomes

$$
E_{m}(z, t)=\varepsilon_{0}(1+\varepsilon \cos \Omega t) \sin \left(\omega_{m} t+\phi_{m}\right) \sin k_{m} z
$$

This field can be written as the sum of harmonically varying fields

$$
E_{m}(z, t)=\varepsilon_{0}\left\{\begin{array}{l}
\sin \left(\omega_{m} t+\phi_{m}\right)+\frac{\varepsilon}{2} \sin \left[\left(\omega_{m}+\Omega\right) t+\phi_{m}\right]+ \\
\frac{\varepsilon}{2} \sin \left[\left(\omega_{m}-\Omega\right) t+\phi_{m}\right]
\end{array}\right\} \sin k_{m} z
$$

Amplitude modulation produces sidebands at $\omega_{m} \pm \Omega$ around the carrier frequency, $\omega_{m}$, of each oscillating mode. By modulating the gain or loss at exactly the longitudinal mode frequency spacing (or the time for light to make a round-trip through the laser cavity)

$$
\Omega=\Delta=\omega_{m+1}-\omega_{m}=\pi c / L
$$

every sideband for every mode will match the adjacent mode frequency. A strong coupling between these matched modes causes them to lock together in phase. Thus, modulating mode amplitudes at the mode separation frequency locks all modes in phase and produces short pulses as described in Section 2.4.2.

Active AM mode locking is commonly achieved by acousto-optic modulators. Acousto-optic modulators diffract light by sound waves, i.e., Brillouin scattering, within the laser cavity. An ultrasonic sound wave is generated by applying a periodic voltage to a piezoelectric crystal and driving a block of quartz. The sound wave is a wave of density, or refractive index, variation in the quartz. The refractive index variation acts as a diffraction grating 
to couple light out of the cavity and produce a periodic loss that, when matched to the cavity round-trip time, locks lasing longitudinal modes in phase so output light is a short pulse.

\subsubsection{Passive AM Mode Locking}

The oscillator laser for the experiments described in this work uses passive Cw AM mode locking to produce ultra-short, $\sim 130 \mathrm{fs}$, pulses. Passive $\mathrm{cw}$ AM mode locking balances saturable absorption and saturable gain pumped by a cw laser. A saturable absorber is an absorbing material which has an absorption coefficient, $q$, that can be saturated by laser radiation according to the formula

$$
q=\frac{q_{0}}{1+I / I^{\text {sat }}}
$$

where $q_{0}$ is the small signal absorption coefficient, $I$ is the laser intensity, and $I^{\text {st }}$ is a characteristic intensity at which absorption is half the small signal absorption. $I^{\text {sat }}$ is usually much greater than $I$, so Eq. (2.16) is approximated by

$$
q \approx q_{0}-q_{0} I / I^{\text {sat }}
$$

Saturable gain follows similar relations. The simplest way to understand passive mode locking is by describing the action of the saturable absorber and saturable gain on the laser pulse in the cavity, as shown in Figure 2.4. Initially, saturable loss is greater than the saturable gain, so the pulse's leading edge experiences absorption. As the pulse is absorbed, the absorber begins to saturate. At some point on the pulse's leading edge, gain becomes greater resulting in net pulse amplification. Gain becomes saturated on the pulse's trailing edge and again drops below absorption. Net absorption then attenuates the pulse's trailing edge. The combination of a saturable gain medium and a saturable absorber amplifies the pulse and shortens it. For steady state mode locking, the saturated media must recover before the pulse makes another pass through the cavity. 


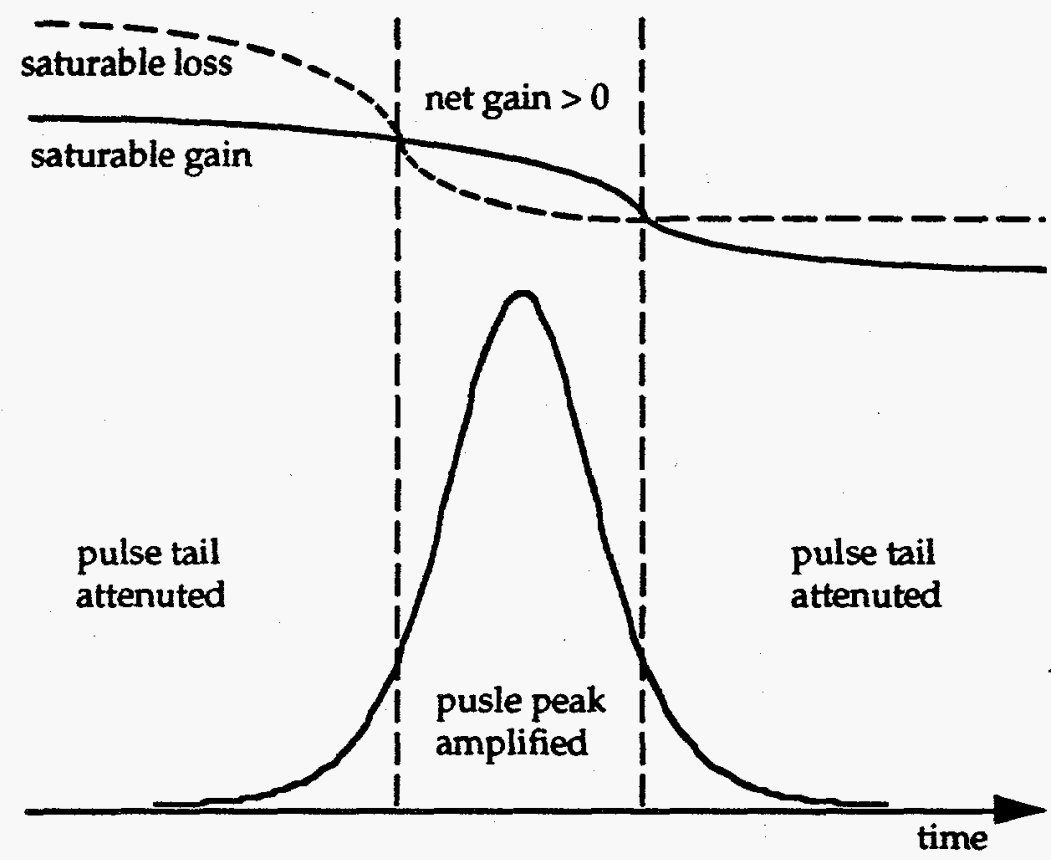

Figure 2.4: Gain and absorber saturation on each pass in a cw modelocked laser.

The theory of $\mathrm{cw}$ mode locking [54-59] leads to a self-consistent differential equation for steady-state pulseshape, $E(t)$, in terms of timedependent saturable gain, $g(t)$, and saturable absorption, $q(t)$

$$
\frac{1}{\omega_{c}^{2}} \frac{d^{2} E}{d t^{2}}-\left(\frac{a_{1}}{\omega_{c}}+\delta T\right) \frac{d E}{d t}+\left[g(t)-q(t)-a_{0}\right] E(t)=0
$$

where $\omega_{c}$ is the carrier frequency, $a_{0}$ is the linear round-trip loss coefficient, $a_{1}$ is a dispersion coefficient and $\delta T$ is the time delay between the round-trip time-offlight and the actual cavity round-trip time. There are similar self-consistent equations for gain and absorption. The solution to Eq. (2.18) gives a pulseshape

$$
\begin{aligned}
& E(t)=E_{0} \operatorname{sech}\left(t / \tau_{p}\right) \equiv \frac{E_{0}}{\cosh \left(t / \tau_{p}\right)} \\
& \text { or } \\
& I(t)=I_{0} \operatorname{sech}^{2}\left(t / \tau_{p}\right) \equiv \frac{I_{0}}{\cosh ^{2}\left(t / \tau_{p}\right)}
\end{aligned}
$$


where $\tau_{p}$ is the pulse full width at half maximum. A comparison (Figure 2.5) of this predicted pulseshape with the measured oscillator pulseshape shows excellent agreement.

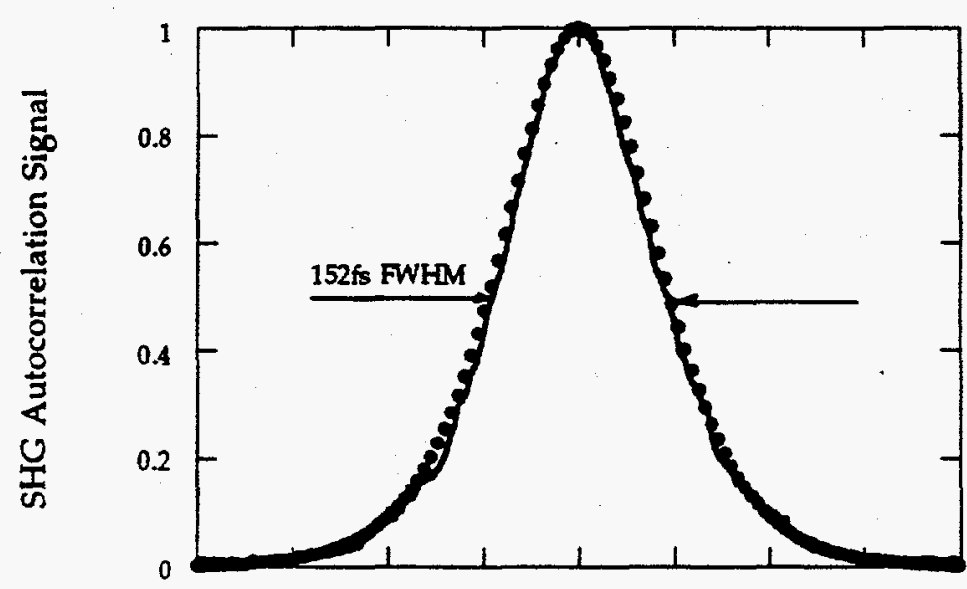

Time Delay

Figure 2.5: Comparison of the CPM measured second harmonic generation (SHG) autocorrelation signal (solid) with the predicted sech ${ }^{2}$ pulseshape (dot) in Eq. (2.19).

\subsubsection{FM Mode Locking}

FM mode locking [16] is not present in the oscillator used in these experiments, but the principles are similar to AM mode locking. By modulating the field phase a series of sidebands is produced, spaced by $\Omega$ and centered about the mode carrier frequencies $\omega_{m}$. Modes and sidebands overlap and come into resonance to produce mode locking when the phase modulation frequency equals the mode separation frequency.

FM mode locking uses electro-optic phase modulators. The index of refraction of a medium is linearly proportional to an applied electric field. The field phase can thus be controlled by application of a voltage across the electro- 
optic medium. A sinusoidal voltage at the longitudinal mode separation frequency mode-locks the laser and short pulses are produced.

\subsubsection{Other Methods of Producing Short Pulses}

The most common alternative technique for producing short pulses is synchronous pumping $[60,61]$. Synchronous pumping uses an actively modelocked pumping source, such as an argon ion or Nd:YAG laser, to pump a passively mode-locked dye laser. The dye has a much larger bandwidth than the pump source, so it can support shorter pulses. The two lasers are synchronized by matching the active mode locking frequency to the passively mode-locked laser repetition rate. This technique also allows shorter pulses without a saturable absorber in the dye laser, because the dye has a greater bandwidth. With actively mode-locked pump pulses of about 40-300ps, synchronously pumped pulses are about 1-30ps [21]. These pulses, however, are not short enough to produce the high-density plasmas studied here, because they allow for significant plasma expansion during pulse interaction. Ideally, plasma expansion should be a small fraction of a wavelength to consider expansion negligible. At a typical plasma expansion velocity of $10^{7} \mathrm{~cm} / \mathrm{s}(1 \AA / \mathrm{fs})$, plasma expansion is $<0.02 \lambda_{\text {leser }}$ if the pulse is $\sim 100 \mathrm{fs}$. The amplified $\mathrm{cw}$ mode-locked dye laser system described in Section $2.5(\sim 3.5 \mathrm{~mJ}, \sim 130 \mathrm{fs}$ pulses) was well suited for these experiments. Moreover, only the dye laser system used in these experiments produced pulses short enough for satisfactory time-resolution of the plasma hydrodynamics described in Chapter 5.

\subsection{THE DYE LASER SYSTEM}

A significant part of the research presented here involved the design, construction, and operation of an amplified CPM dye laser system. The design was based largely on a dye laser system used by Murnane [62], but with several 
improvements and modifications [17]. Amplified pulses as short as 75fs and with energy exceeding $4 \mathrm{~mJ}$ could be obtained, but the most reliable, stable operation generated $\sim 130 \mathrm{fs},-3.5 \mathrm{~mJ}, 614 \mathrm{~nm}$ pulses at a repetition rate of $10 \mathrm{~Hz}$.

\subsubsection{The CPM Oscillator}

The CPM oscillator shown in Figure 2.6 was designed by Clark Instrumentation, Inc. (model CPM-1) and constructed for this research. The design is based on the dispersion compensated version of the original CPM ring dye laser developed by Fork [25]. Original CPM lasers relied mainly on saturable absorption and saturable gain and were unpredictable, unstable, and unreliable. Although pulses shorter than 100 fs could be obtained, the pulseshape tended to contain appreciable amounts of energy in the pulse wings, so its experimental use was limited.

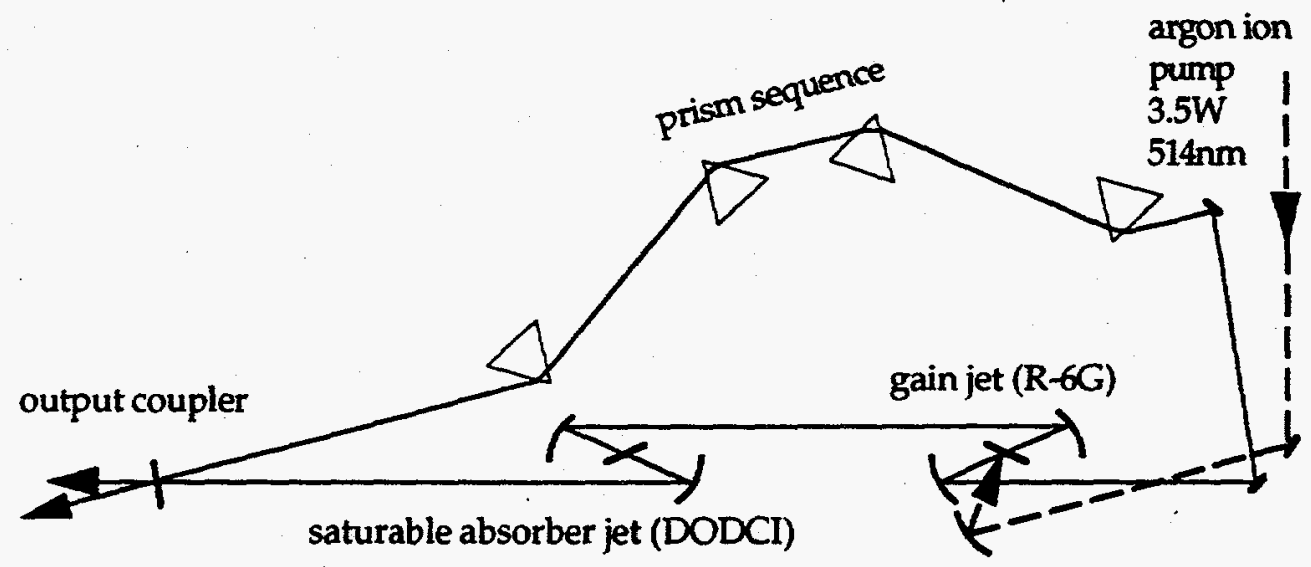

Figure 2.6: The CPM laser oscillator.

The dispersion compensated CPM dye laser used in this research was developed between 1982 and 1985. It goes beyond just using saturable gain and absorption by employing SPM to create bandwidth and dispersion compensation to minimize the deleterious effects of phase dispersion [63] over a wide bandwidth. 


\subsubsection{Gain}

The gain medium is the laser dye rhodamine-6G (R-6G) dissolved in an ethylene glycol solution ( $5 \times 10^{-3}$ molar) and pumped at $=40 \mathrm{psi}$ through a vertical jet nozzle $(300 \mu \mathrm{m}$ thick by $3 \mathrm{~mm}$ wide). The dye circulation system is shown in Figure 2.7.

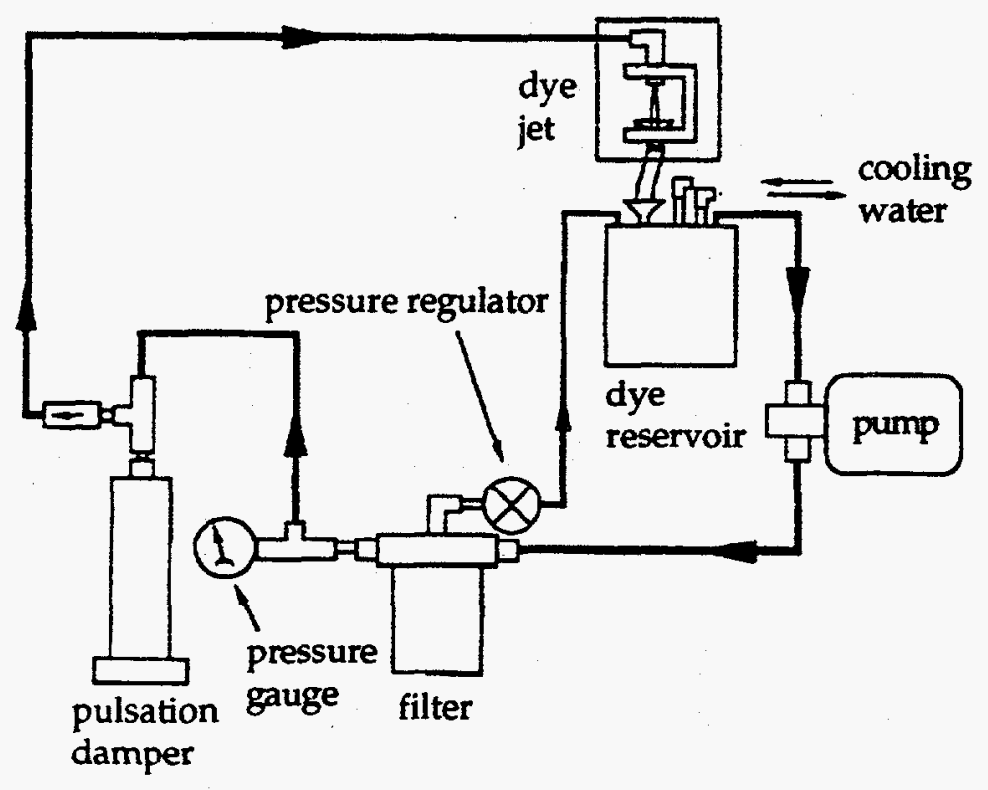

Figure 2.7: The dye circulation system for the gain and saturable absorber dyes in the CPM dye laser oscillator.

An $80 \mu \mathrm{m}$ thick, optically flat sheet of dye is produced [63]. Ethylene glycol solvent viscosity assures a laminar flow necessary for an optical quality element in the laser cavity. The lasing beam is focused onto the gain medium to a spot size of $10 \mu \mathrm{m}$ (in TEM 00 mode) by two spherical mirrors with $10 \mathrm{~cm}$ radius of curvature. The gain jet is placed at Brewster's angle $\left(\approx 55^{\circ}\right)$ to minimize reflection losses as pulses propagate around the cavity. A population inversion in the dye is achieved by focusing with a separate $10 \mathrm{~cm}$ mirror approximately $3.5 \mathrm{~W}$ of 
$514 \mathrm{~nm}$ light from a Coherent model Innova $200-20 \mathrm{cw}$ Argon ion ( $\left.\mathrm{Ar}^{+}\right)$laser. Pumping at $514 \mathrm{~nm}$ overlaps well with the R-6G absorption curve peak.

The amplifying action of $\mathrm{R}-6 \mathrm{G}$ dye molecules in a liquid solvent occurs when an electronic population inversion is created between the first excited singlet level, $\mathrm{S}_{1}$, and thermally populated ground state rovibronic levels, $\mathrm{S}_{0}$, as shown in Figure 2.8 [64]. Collisions with the solvent cause dye molecules excited by laser pumping to high vibrational levels in $S_{1}$ to relax to the lowest vibrational level, $v_{0}$, in fast $\left(10^{-11}-10^{-12} \mathrm{~s}\right)$, radiationless transitions. The $\mathrm{v}_{0}$ level depopulates by spontaneous and stimulated emission to rovibronic levels of $S_{0}$ or by intersystem crossing to a triplet state, $T_{1}$. These transitions are generally lower in energy than those caused by excitation, so the fluorescence spectrum is redshifted from the absorption spectrum. Lower level, $v_{\mathbf{k}}\left(\mathrm{S}_{\mathbf{0}}\right)$, population created by stimulated emission is rapidly drained by collisions with solvent molecules. The entire laser population scheme can be approximated by a classical four-level laser system with lasing transition $\mathrm{v}_{0}\left(\mathrm{~S}_{1}\right) \rightarrow \mathrm{v}_{\mathrm{k}}\left(\mathrm{S}_{0}\right)$. Strong interaction between dye and solvent molecules also causes collisional fluorescence line broadening. . Lines are broadened so much that discrete spectral lines overlap and form a continuous spectrum over which the dye laser can be tuned.

Intersystem crossing to triplet states, $T_{1}$, reduces the population inversion and the triplet absorption spectrum may partially overlap the singlet fluorescence spectrum causing additional losses. In the CPM, these problems are overcome by flowing the dye rapidly through the excitation region in liquid jets. At a flow velocity of $10 \mathrm{~m} / \mathrm{s}$, the time-of-flight for the dye molecule through a $10 \mu \mathrm{m}$ focus is about 1 $1 \mu \mathrm{s}$ [64] which is much shorter than the triplet state lifetime, so the triplet population is minimized. 


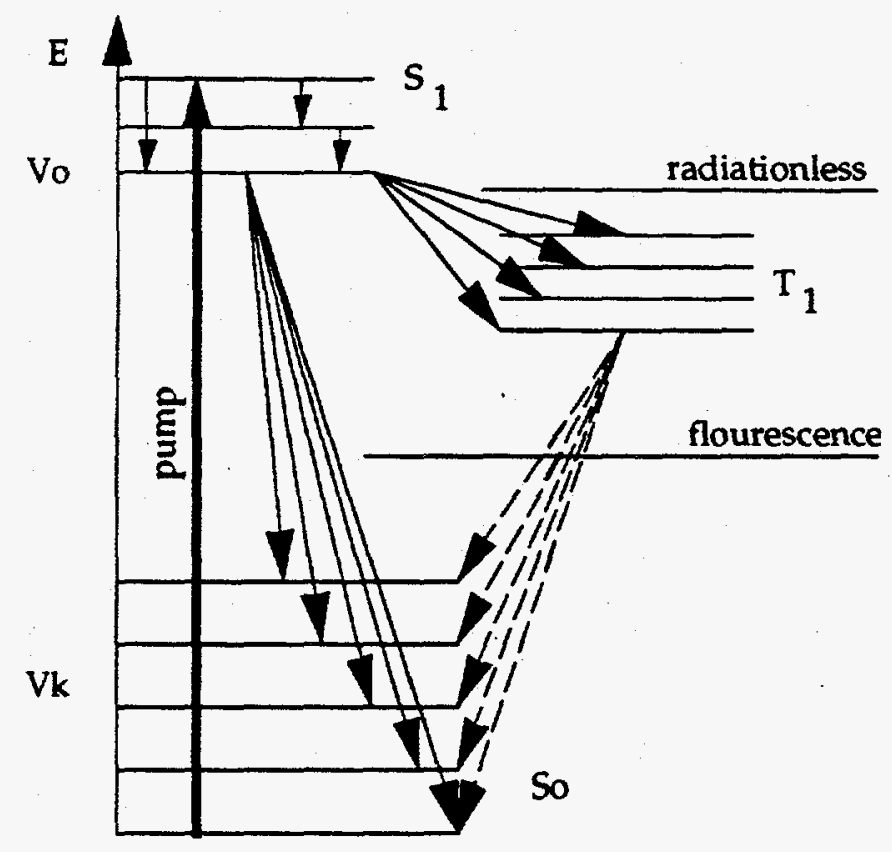

Figure 2.8: Schematic energy level diagram and pumping cycle in $R-6 G$ dye (after [64]).

\subsubsection{Saturable Absorber}

The saturable absorber is a $15 \mathrm{psi}$ dye jet of $3.3^{\prime}$ diethyloxadicarbocyanine iodide (DODCI) dissolved in ethylene glycol ( $5 \times 10^{-4}$ molar solution). The jet nozzle is about $120 \mu \mathrm{m}$ thick and $3 \mathrm{~mm}$ wide giving a saturable absorption region of about $50 \mu \mathrm{m}$. The jet is positioned at the focus of two spherical mirrors with $5 \mathrm{~cm}$ radii of curvature. $A T^{T} M_{00}$ mode is focused to a waist of $5 \mu \mathrm{m}$. The saturable absorber jet is placed at a negative Brewster's angle $\left(=55^{\circ}\right)$ to the beam to minimize cavity losses from reflection and compensate for astigmatism induced by focusing into the angled gain jet.

The saturable absorber and gain jets produce passive mode locking as described in Section 2.4.4. Solvent in the saturable absorber also creates extra bandwidth necessary for stable, ultra-short pulses. A material with a positive nonlinear index of refraction produces positive SPM in gain saturation and negative SPM in absorption saturation. Thus, SPM due to saturation of the R-6G 
and DODCI approximately balances out. Extra bandwidth is created by SPM from the interaction of high peak power intracavity pulses with the ethylene glycol solvent in the DODCI saturable absorber dye. This interaction is not significant unless both pulses are simultaneously present in the absorber, as in a CPM. Their combined fields heavily saturate the absorber during their leading edges, so the remaining pulse energy produces SPM in the ethylene glycol solvent. The specific process for this SPM is the fast electronic Kerr effect in the ethylene glycol molecules. Heavy absorber saturation from the collision of the two counter-propagating pulses is also the lowest laser loss mode, so the laser will always run such that SPM takes place in the ethylene glycol.

Ethylene glycol solvent has a large nonlinear index of refraction, so it induces a net positive SPM on the pulse in the following manner $[21,28,30]$. In general, the polarization $P$ of a material can be expanded as a power series in the electric field $E$

$$
P=\chi_{1} \varepsilon_{0} E+\chi_{2} E^{2}+\chi_{3} E^{3}+\ldots
$$

where $\varepsilon_{0}$ is the free space dielectric constant, $\chi_{1}$ is the linear susceptibility, $\chi_{2}$ and $\chi_{3}$ are the second and third order susceptibilities. It can be shown that all nonlinearities of even order are zero for centrosymmetric crystals [28]. Liquid ethylene glycol acts as if it has a center of symmetry, so we ignore these even terms. Electric displacement in the ethylene glycol can then be written to third order in the field as

$$
d=E\left(\varepsilon_{0}\left(1+\chi_{1}\right)+\chi_{3} E^{3}\right)
$$

and the total (linear and nonlinear) dielectric constant is

$$
\varepsilon=\varepsilon_{1}+\varepsilon_{2} E^{2}
$$

or the total index of refraction is

$$
n=n_{0}+n_{2} E^{2}
$$


(using $d=\varepsilon E$ and $n=\sqrt{\varepsilon / \varepsilon_{0}}$ ) where $n_{2} E^{2}$ is the nonlinear index of refraction variation. Eq. (2.23) shows how the index of refraction in the ethylene glycol depends on the intensity, $\sim E^{2}$. This relation leads to generation of extra bandwidth from SPM $[31,63,65-73]$. The time-dependent index of refraction is

$$
\Delta n(t)=n_{2} E^{2}(t) \sim n_{2} I(t)
$$

so the change in the pulse phase from interaction with the pulse, i.e., the selfphase modulation is

$$
\Delta \phi(t)=(\omega / c) \Delta n(t) l=(\omega / c) n_{2} E^{2}(t) l
$$

and the frequency modulation is

$$
\Delta \omega(t)=-\partial(\Delta \phi) / \partial t
$$

A rigorous derivation [30] gives the complete SPM output spectrum as

$$
|E(\omega)|^{2}=\left|\int_{0}^{\infty} \mathrm{E}(t) \exp \left(-i \omega_{0} t+i \Delta \phi(t)\right) d t\right|^{2}
$$

From Eq. (2.27) the output spectrum follows the pulseshape [30]. It broadens symmetrically for a symmetric pulse and asymmetrically for an asymmetric pulse. The highest and lowest frequencies generated occur at the points of maximum slope in the intensity or $\Delta \phi(t)$ curve, the inflection points. A semiperiodic structure in the spectrum is generated by interference between generated waves with the same frequency, but different phases. Finally, the SPM spectrum will shift from red to blue frequencies as a function of time. Since $n_{2}>0$ in ethylene glycol, $\Delta \omega<0$ during the pulse's rising edge and $\Delta \omega>0$ during the pulse's falling edge with the maxima and minima at the pulse inflection points. A pulse acquires an approximately linear chirp across the pulse's central region as shown in Figure 2.9. 

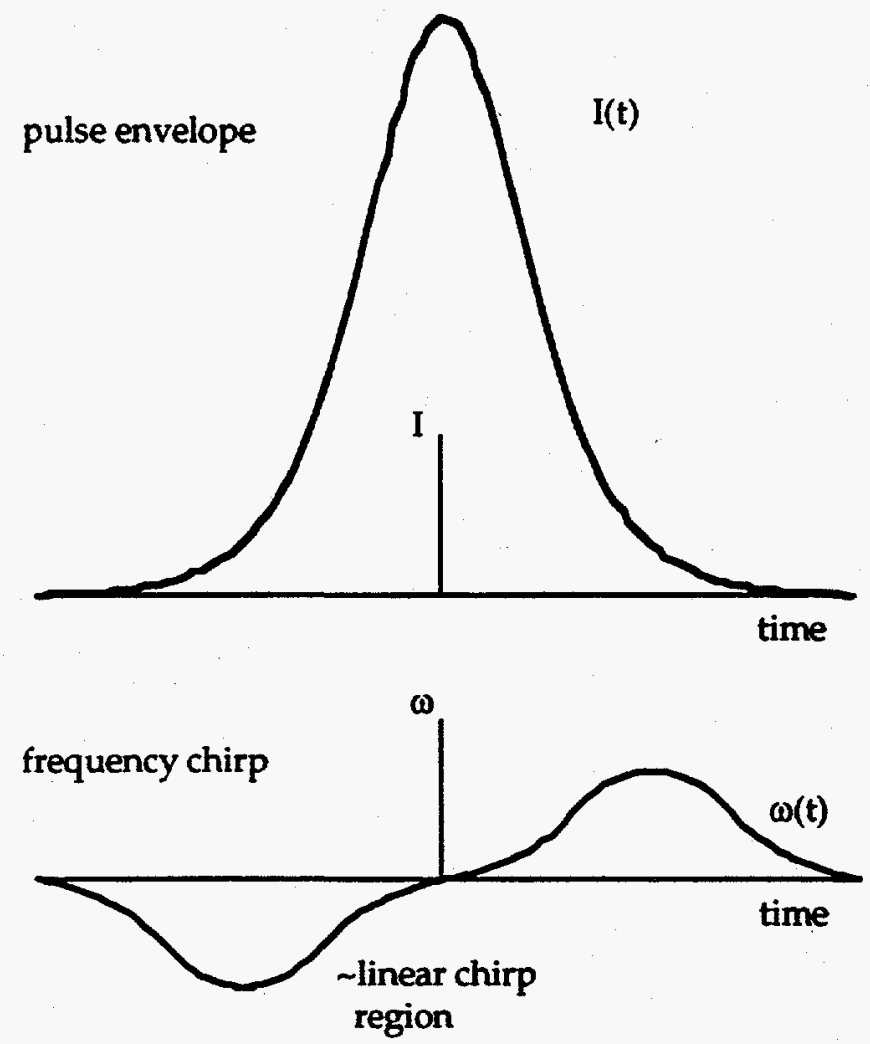

Figure 2.9: Chirped pulse resulting from SPM.

Besides the chirp, the pulse also experiences group velocity dispersion, $d v_{g}(\omega) / d \omega$, where $v_{g}(\omega)$ is the pulse group velocity. This dispersion is a result of different frequencies seeing different optical densities and therefore traveling at different speeds. The pulseshape changes proportionally with distance by this linear frequency spreading. Dispersion also causes a chirp, but it is only a redistribution of the pulse frequencies. SPM creates new frequencies to increase total pulse bandwidth and allow pulse compression.

The CPM's combined dispersion and SPM produces pulses with solitonlike behavior [1, 54]. Solitons are particular solutions to a nonlinear wave equation such as the nonlinear Schrödinger equation [1]. Each solution is characterized by a related amplitude and pulseshape. The pulseshape can propagate with an unchanging shape indefinitely. Solitons occur if the nonlinearity ( $n_{2}$ in our case) is positive and the dispersion is negative, or vice 
versa. A soliton solution for the nonlinear Schrödinger equation in the CPM laser is

$$
E(z, t)=E_{0} \operatorname{sech}\left(\frac{t-t_{0}-z / v_{8}}{\tau_{0}}\right) \exp [-i(\Omega t-\kappa z)]
$$

where $E_{0}$ is the amplitude, $\tau_{0}$ is the pulsewidth, $v_{g}$ is the group velocity and $\Omega$ and $\boldsymbol{x}$ are frequency and wavenumber shifts, respectively. The measured second-order autocorrelation pulseshape agrees with this theoretical shape as shown in Figure 2.5.

\subsubsection{Dispersion Compensation in the CPM}

Since positive chirp in the CPM pulses is mostly linear as shown in Figure 2.9, pulse compression is easily produced by a set of four intracavity prisms designed by Fork [36, 74] (Figure 2.10).

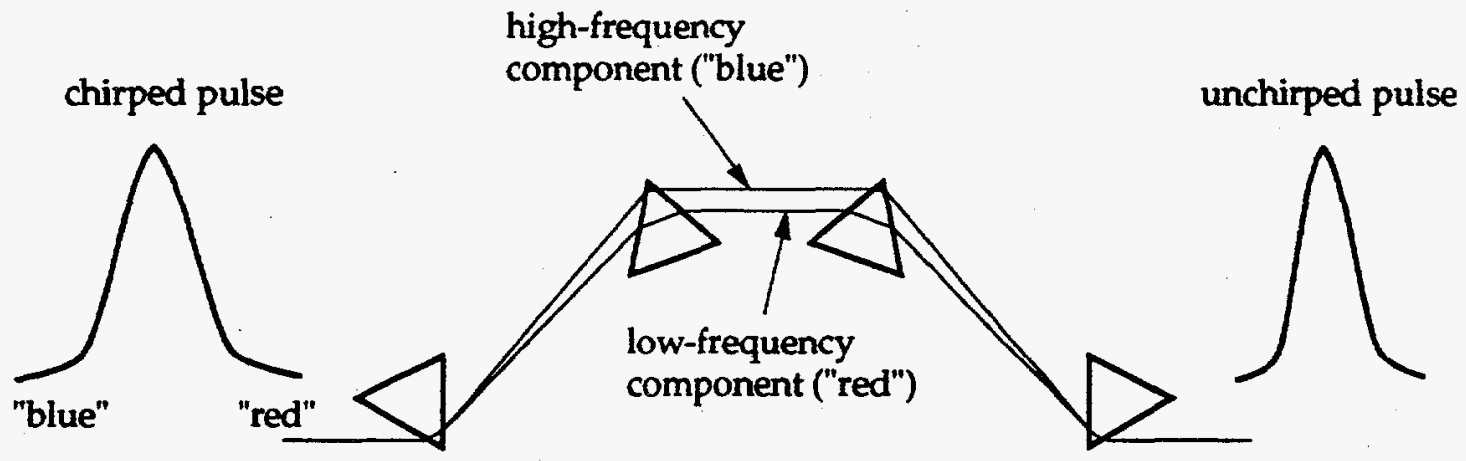

Figure 2.10: The 4-prism sequence in the CPM cavity compresses the pulse by forming a longer optical path for leading lower frequencies. Trailing higher frequencies catch up and linear chirp is remooed.

The four prisms are set at the angle of minimum deviation in a geometry that allows continuous adjustment of group velocity dispersion (GVD) through positive and negative values without beam displacement $[36,74]$. Physically this means that the prism geometry forces red light on the pulse's leading edge to 
travel a longer optical path length than blue light on the pulse's trailing edge. The path length difference between colors in the pulse is proportional to the wavelength difference in the pulse bandwidth, so all linear chirp can be compensated for.

\subsubsection{Pulse Propagation Around the Cavity}

Using the preceding discussion of essential CPM processes, we can now describe basic, steady-state operation by looking at pulses propagating around the cavity [75]. Initially, two counter-propagating pulses build up from noise penetrating the saturable absorber as described in Section 2.4.4. As a pulse passes through the DODCI, the leading edge is absorbed as it saturates the absorber, so the pulse narrows. A small negative chirp from absorption saturation is also imposed on the pulse. The remaining pulse energy interacts nonlinearly with the ethylene glycol to produce positively chirped SPM. The clockwise $(\mathrm{cw})$ traveling pulse partially transmits through the output coupler to form the first CPM beam.

The counterclockwise (ccw) pulse, meanwhile, is amplified in the gain jet (R-6G) and obtains a small positive chirp from gain saturation. Since the gain medium saturates near the pulse center, the trailing edge is not amplified and the pulse becomes shorter. The ccw pulse experiences positive GVD in the four prisms, mirrors, and air, but this is overcompensated by the prism arrangement that forces the red frequencies along a longer optical path than the blue. The net effect is negative GVD that balances positive SPM chirp and positive intracavity dispersion chirp. Pulse compression also results from the negative GVD because of extra bandwidth created by SPM. The ccw pulse is then partially transmitted through the output coupler to form the second laser beam. 
The $\mathrm{cw}$ pulse also goes through the prism/SPM compression and reaches the gain jet about $5 \mathrm{~ns}$ after the ccw pulse. The continuously pumped gain medium has fully recovered by then and amplifies this pulse while imposing a small positive chirp as with the $\mathrm{ccw}$ pulse. The small positive and negative chirps in the dye jets nearly cancel and the main process for pulse compression is the balance of negative prism GVD and positive SPM. Note that the prism sequence can be adjusted to produce a net positive GVD, but stable solutions no longer exist and the CPM does not produce mode-locked pulses.

\subsubsection{Typical Operating Parameters}

The CPM output was typically a pulse train with repetition frequency of about $90 \mathrm{MHz}$ defined by the approximately 11 ns cavity round-trip time. Each pulse had an energy of about 200pJ, a pulsewidth of 150fs, and a bandwidth of about $4.37 \mathrm{~nm}$ centered near $616 \mathrm{~nm}$ (Figure 2.11 ). The greatest long-term stability was achieved when the laser mode was near TEM00. The pulsewidth and spectrum were measured at various points in the parameter space in which the CPM typically operated and the time-bandwidth product was then calculated. The combination of pulsewidth, spectrum, and laser mode mentioned above was shown to also produce pulses closest $(1.7 \mathrm{x})$ to transform limit for a sech ${ }^{2}$ pulse shape, $\Delta v \Delta \tau=0.315$, as shown in Figure 2.12 . 

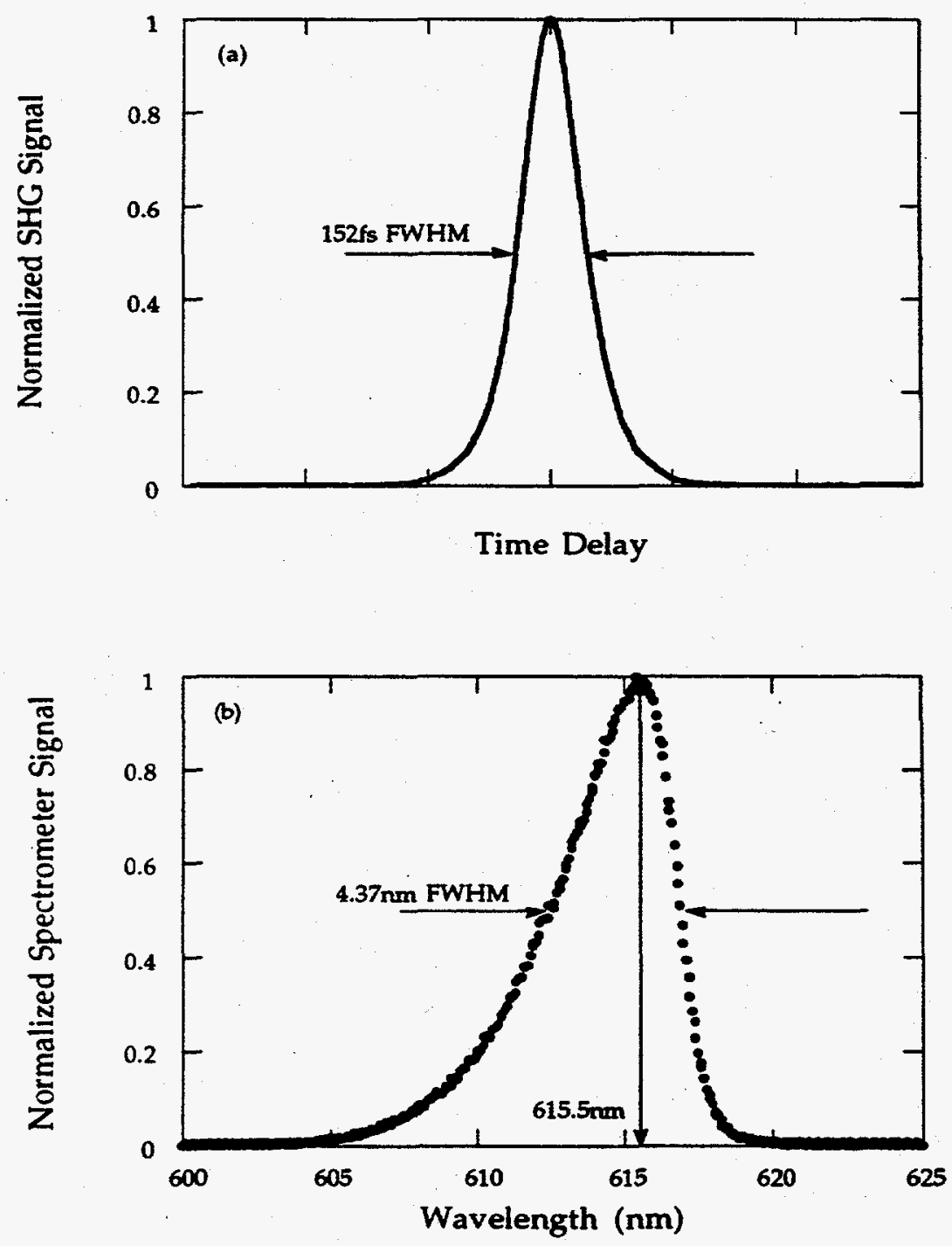

Figure 2.11: The CPM (a) pulse and (b) spectrum. 


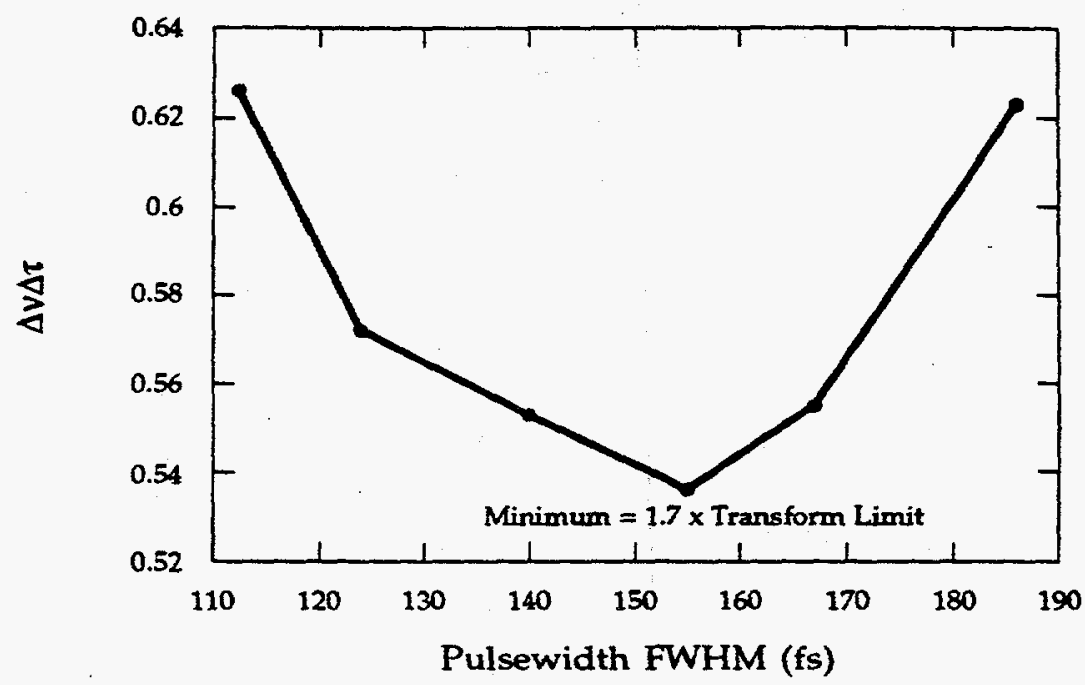

Figure 2.12: The CPM typically operates near $1.7 x$ transform limit.

\subsubsection{The Argon Ion Oscillator Pump Laser}

$\mathrm{A} \mathrm{Cw}$ argon ion $\left(\mathrm{Ar}^{+}\right)$gas laser (Coherent Lasers model Innova 200-20) was used to excite the CPM oscillator gain medium (R-6G). In the $\mathrm{Ar}^{+}$laser, lasing occurs between highly excited energy levels in singly ionized atoms. Excitation occurs through electron impact driven by high current dc discharges across the laser tube. Atoms are ionized in one collision and brought to highly excited states by subsequent collisions. Ions then relax, cascading to lower states in radiating transitions along a number of different energy channels (see Figure 2.13).

The upper laser levels' lifetime is $~ 5 \mathrm{~ns}$. Population in the lower laser levels rapidly decays to the ground state where ions recombine to form neutral atoms. Rapid lower laser level depopulation minimizes competition between different laser transitions. A single laser line is selected by an intracavity prism. The prism refracts lasing lines so only one laser line experiences gain, while the rest are directed out of the cavity. 


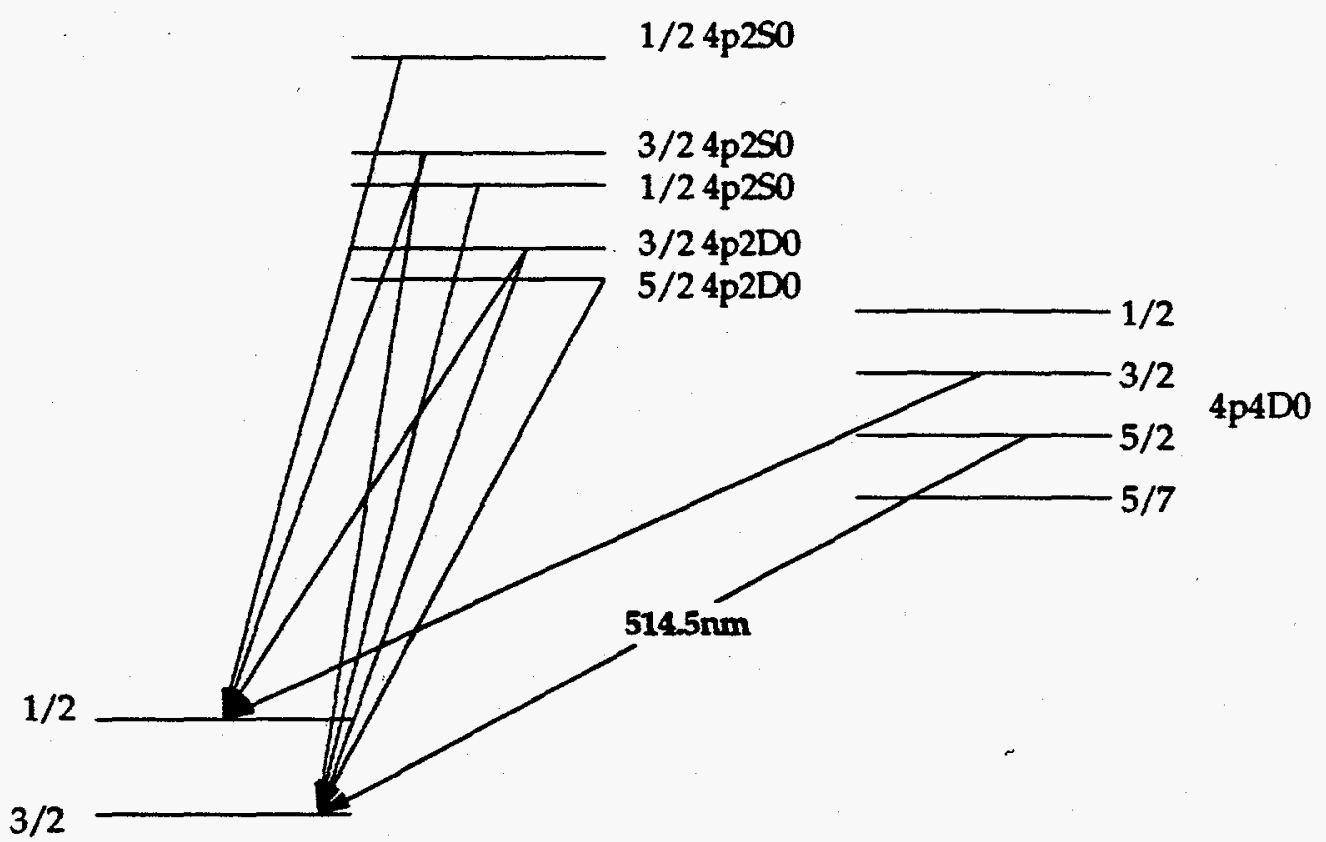

Figure 2.13: Energy leoels and radiatioe pathways of the 4p-4s argon ion laser transitions.

Ion laser lines are typically $\sim 4 \mathrm{GHz}$ wide which includes about 20 longitudinal modes and results from several broadening mechanisms. The principle broadening comes from inhomogeneous Doppler broadening and Zeeman splitting. Additional homogeneous broadening comes from the upper laser states' natural lifetimes. An intracavity etalon can be added to select a single frequency from the laser lines with an efficiency of more than $50 \%$, but this was not used in these experiments.

\subsubsection{The Amplifier System}

\subsubsection{Amplifier System Design and Layout}

The amplifier system was designed for maximum energy output without significant degradation of the CPM laser spatial and temporal coherence. It was also designed to keep prepulse, due to ASE in the amplifiers, below the target damage fluence. Moreover, requirements for high-quality amplification of high- 
power pulses discussed in Section 2.2 were integrated into the design. Dyes were used as the amplifying medium, because they have a bandwidth wide enough to uniformly amplify all spectral parts of the pulse. Dyes also have a large saturation fluence $\left(\sim \mathrm{mJ} / \mathrm{cm}^{2}\right)$, so a large amount of energy could be obtained before significant ASE was produced. Finally, nonlinear phase shift was minimized by using progressively larger channel diameters to reduce the intensity seen by the amplifiers. Beam expanding telescopes between successively higher-power amplifier stages, combined with spatial filters, removed both ASE and amplitude ripples caused by optics imperfections. Amplitude ripples were composed primarily of high spatial frequencies that are scattered into large angles and, therefore, suppressed when the beam was spatially filtered.

The dye amplifier system shown in Figure 2.14 consisted of a six-pass bow-tie amplifier [76-78] (BTA) shown in Figure 2.15 followed by two Bethune cell [79] dye amplifiers with bore diameters of $6 \mathrm{~mm}$ and $20 \mathrm{~mm}$. The BTA raised 200pJ CPM energy output to $\sim 20 \mu \mathrm{J}$. The BTA design allowed the CPM pulse to see gain each time it passed through a dye cell pumped by a $\sim 10 \mathrm{~ns}$ Nd:YAG pulse. A malachite green saturable absorber was placed between the fourth and fifth passes to suppress ASE energy to $<1 \%$. The BTA was designed to be pumped by a high-repetition-rate $(\sim \mathrm{KHz})$ copper vapor laser, but was implemented here, for the first time, using a Nd:YAG pump source which operated at $10 \mathrm{~Hz}[17]$. 


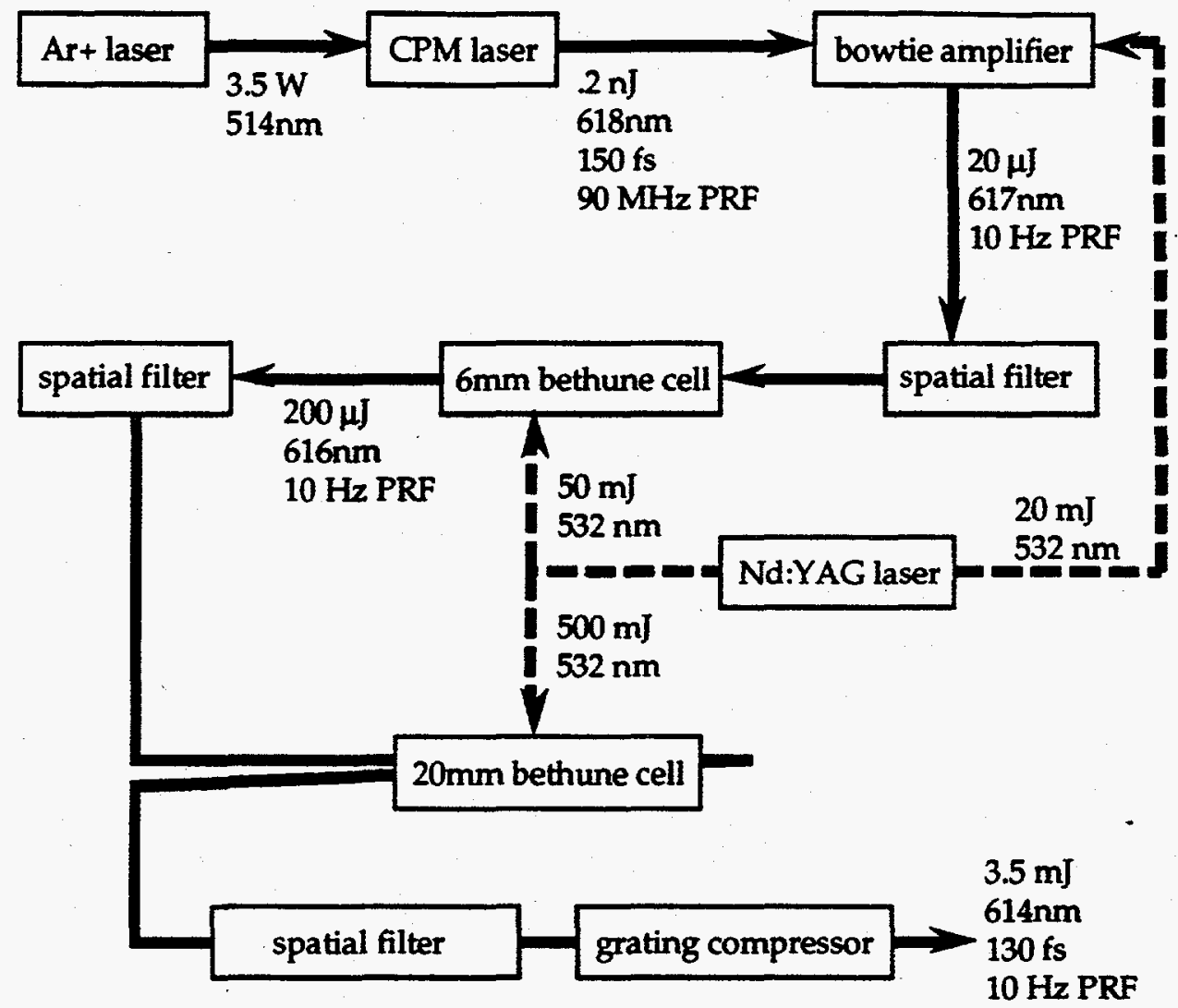

Figure 2.14: Schematic dye laser oscillator, amplifier, and pulse compression system.

BTA pump energy was derived by doubling residual $1.064 \mu \mathrm{m}$ light which leaks through the doubling crystal (used to double the pump light for the Bethune amplifiers) in a second doubling crystal. This pump light was then split by a $50 / 50$ beamsplitter and one pulse was delayed by several nanoseconds before focussing in the dye cell. The combination of direct and delayed pump pulses allowed uniform pumping over the entire CPM seed pulse transit time. By pumping in this manner, a single (Nd:YAG) laser was used for the entire system and the BTA pump energy was essentially independent from the Bethune dye cell pump, which left more pump energy for the Bethune amplifiers. 


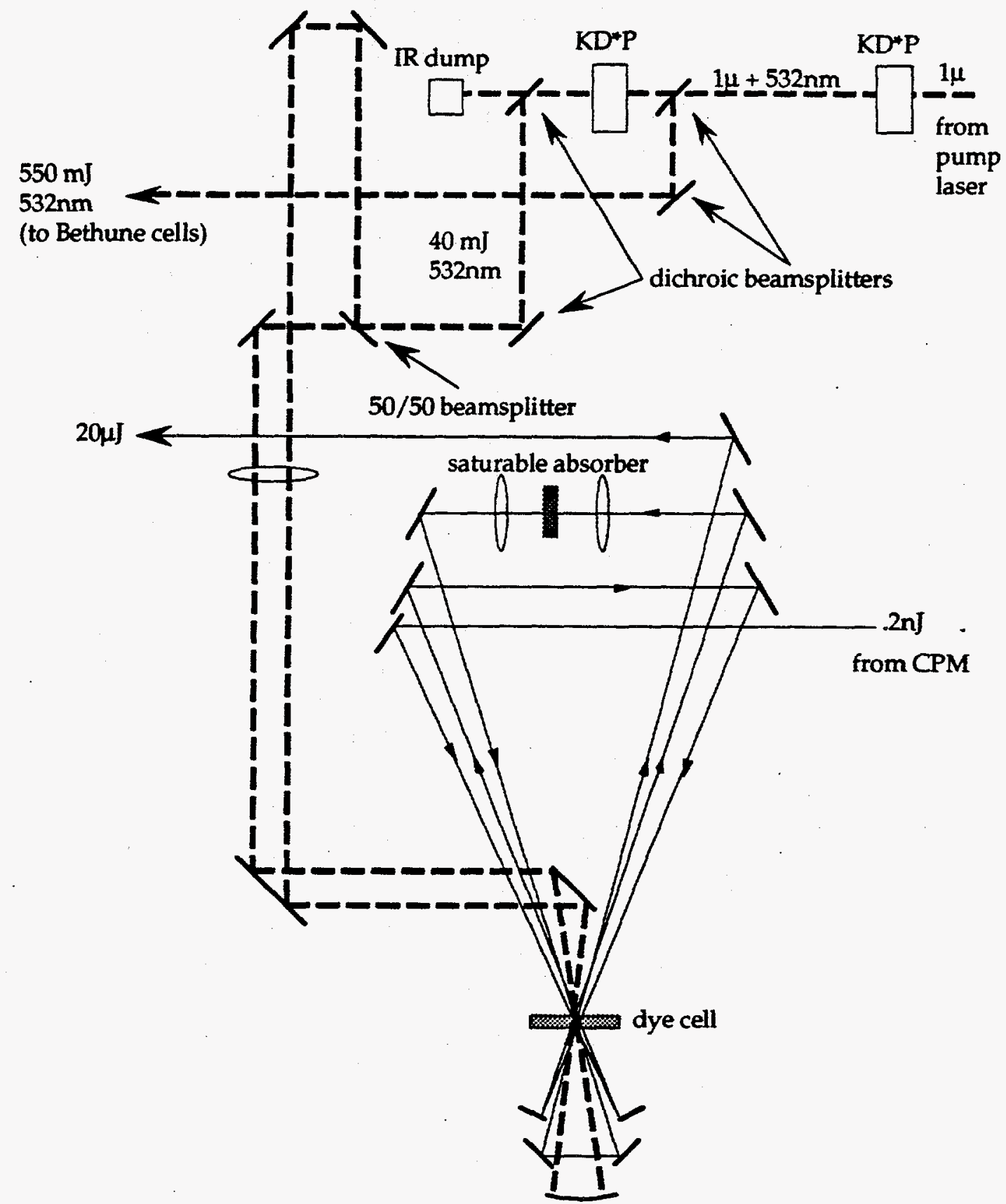

Figure 2.15: The 6-pass bow-tie amplifier and Nd:YAG pump source.

Following the BTA was a pinhole spatial filter used to suppress the ASE from the high-gain BTA even further and provide a clean spatial profile for injection into the Bethune amplifiers. The uniformly illuminated pinhole provided a near flat-topped spatial profile that was relay imaged through the Bethune cells for amplification. 
Relay imaging [21], shown in Figure 2.16, uses the position and focal length of lens pairs to simultaneously achieve a desired beam magnification, $\mathrm{M}=\mathrm{f} 2 / \mathrm{f} 1$, and imaging from an object plane located at $-\mathrm{d} 1$ to an image plane located at $+\mathrm{d} 2$. By this method, the beam profile at the first amplifier center could be imaged as an essentially identical, but magnified, beam profile in the second amplifier center.

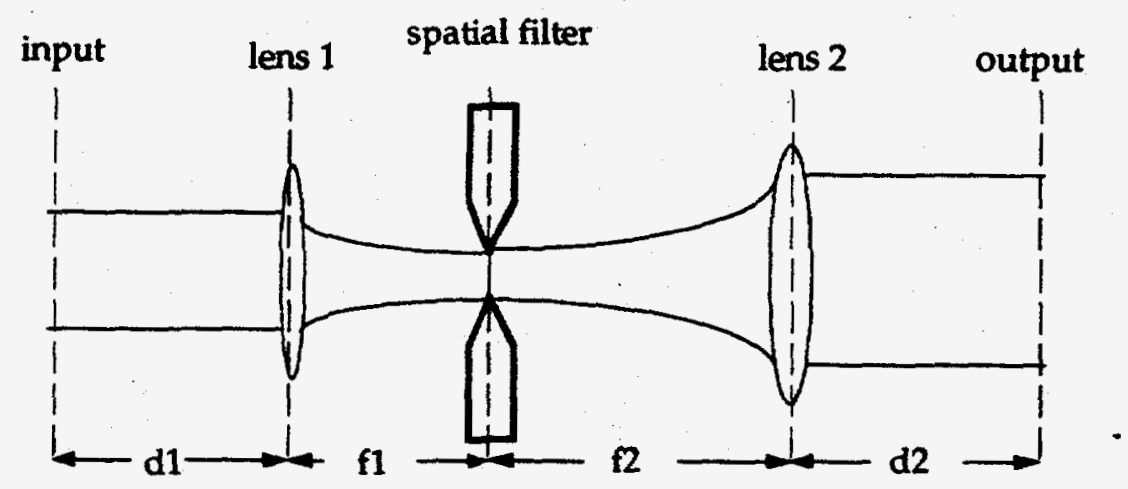

Figure 2.16: Relay imaging with a pinhole provides magnification, spatial filtering and image relaying.

The Bethune amplifiers shown in Figure 2.17 were made by boring a cylindrical channel in a prism for the amplifying dye and oscillator seed beam. This geometry insured dye was uniformly illuminated by transverse Nd:YAG laser pumping.

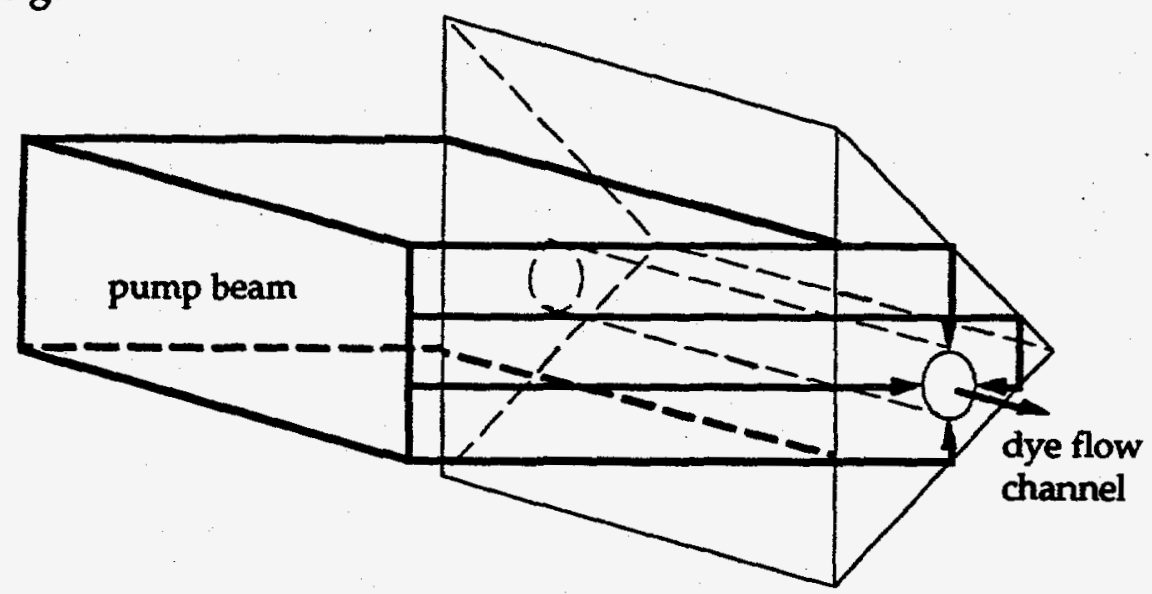

Figure 2.17: The uniform illumination in the Bethune cell amplifier insured amplified pulses with good spatial quality even if the pump beam was not completely uniform. 
The $6 \mathrm{~mm}$ Bethune cell amplified the beam from $-20 \mu \mathrm{J}$ to $>200 \mu \mathrm{J}$. Following the first vacuum spatial filter, the energy was $\sim 200 \mu \mathrm{J}$ with $<0.01 \%$ ASE energy. This second spatial filter was placed in vacuum to avoid SPM in the air as the beam focused through the pinhole. The criterion for significant SPM in air is an intensity of $I_{S P M}=\lambda / 2 \pi m_{2} L[28]$, where $n_{2}$ is the nonlinear refractive index (for air $\left.\mathrm{n}_{2}=4.74 \times 10^{-19} \mathrm{esu}\right), \lambda$ is the wavelength $(0.614 \mu \mathrm{m})$ and $L$ is the medium length (the confocal parameter $=2.68 \times 10^{4} \mu \mathrm{m}$ ). This gives an intensity of $I_{S P M}=7.69 \times 10^{12} \mathrm{~W} / \mathrm{cm}^{2}$. The focused pulse's peak intensity, having power $P$ and spot size $w_{0}$ after the $6 \mathrm{~mm}$ Bethune cell, was about [21] $I=2 P / \pi w_{0}^{2}=2.43 \times 10^{13} \mathrm{~W} / \mathrm{cm}^{2}$ (using a pulsewidth of $200 \mathrm{fs}$, energy of $200 \mu \mathrm{J}$, and waist spot diameter of $51.17 \mu \mathrm{m})$. This is well above the intensity threshold for significant SPM, making a vacuum spatial filter necessary. The $20 \mathrm{~mm}$ Bethune cell was double-passed with beam separation achieved by polarization using a 1/4-waveplate and thin-film polarizer. A 1/2-waveplate and polarizing beam splitting cube were used for continuous adjustment of the final beam energy and to insure the polarization was $>98 \%$ linear. Linear dispersion of the dyes, optical glass, air, and mirrors was then compensated by a pair of compression gratings [35] with $>80 \%$ efficiency. Pulse compression with gratings, Figure 2.18, works in a manner similar to prism compression in the CPM (and in fact can be done with prisms). Note that a single-pass grating compression leaves a spatial chirp on the beam. Gratings have the advantage of being more compact than prisms, because they are more dispersive. Also, because they are reflective, additional glass dispersion found in prisms does not need to be compensated. Generally, the amplified pulse has a positive (red leads blue), primarily linear chirp. Gratings reflect higher frequencies at smaller angles that forces them along a longer path length than the lower frequencies. This allows trailing higher frequencies to catch up to leading lower frequencies. SPM 
can add nonlinear chirp to the pulse that cannot be compensated by the gratings. SPM in the amplifier system was kept to a minimum by expanding the beam as it was amplified to reduce fluence in dyes and optics.

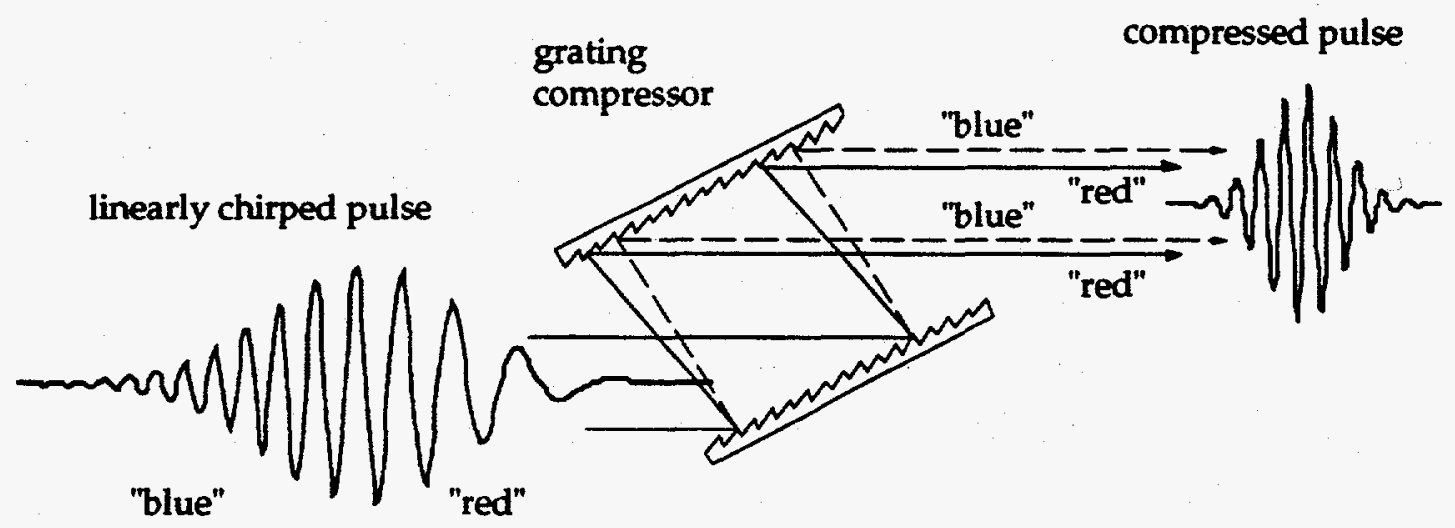

Figure 2.18: The laser system linear dispersion was compensated by a grating pulse compressor.

\subsubsection{Amplifier Analysis}

The most fundamental short pulse amplification analysis involves solving two coupled, nonlinear radiation transfer equations [80-82]

$$
\frac{\partial N(z, t)}{\partial t}=-\frac{2 \sigma}{h v} N(z, t) I(z, t)
$$

and

$$
\frac{\partial I(z, t)}{\partial z}+\frac{1}{c} \frac{\partial I(z, t)}{\partial t}=\sigma N(z, t) I(z, t)
$$

$N$ and $I$ are the instantaneous population inversion and laser intensity, respectively, $\sigma$ is the stimulated transition cross section, $h$ is Planck's constant, $c$ is the speed of light, $v$ is the laser frequency and $z$ and $t$ are the space and timedependent variables. Eq. (2.29) is the population inversion equation derived from rate equations for a two level amplifier system. Eq. (2.30) is the plane-wave equation for intensity in a medium with gain.

In the simplest analysis, a rectangular pulse is considered. The pulse can saturate the gain by reducing the population inversion, but the pulse shape is not 
changed significantly by this saturation. In this case amplified energy from Eqs. $(2.29)$ and $(2.30)$ is $[16,43]$

$$
E_{\text {out }}=E_{\text {sat }} \ln \left[1+e^{g_{0} 0}\left(\exp \left(\frac{E_{\text {in }}}{E_{\text {set }}}\right)-1\right)\right]
$$

where $E_{\text {in }}, E_{\text {out }}$ and $E_{\text {sat }}$ are the input, output, and saturation energy, respectively $l$, is the amplifying length, and $g_{0}$ is the small signal gain coefficient. $g_{0}$ can be modeled for a transversely pumped amplifier by [83]

$$
g_{0} \approx \frac{\xi \pi E_{p} \sigma_{e m} \tau_{i n v}}{t_{p} h \omega_{p} 4 r^{2}}
$$

where $\xi$ is the dye efficiency, $E_{p}$ is the pump energy, $\sigma_{e m}$ is the gain dye emission cross section, $\tau_{i n v}$ is the dye inversion lifetime, $t_{p}$ is the pump pulse FWHM, $\omega_{p}$ is the pump laser frequency, and $r$ is the dye cell radius.

Eq. (2.31) becomes less valid when the amplified pulse shape differs significantly from the incident pulse shape, because higher gain is experienced by the pulse's leading edge [43]. A real pulse will saturate the population inversion, so one must account for the pulse tail seeing a population inversion that has been partially saturated by the leading edge $[21,84,85]$.

\subsubsection{Amplified Laser Output Characteristics}

The amplified laser system output is shown in Figure 2.19. The laser pulsewidth was $\sim 130 \mathrm{fs}$. The spectral width was $\sim 5.9 \mathrm{~nm}$.

An important laser pulse characteristic for these experiments is the ratio of peak intensity to intensity of the energy pedestal surrounding the main pulse in time. This figure is termed the contrast ratio. As discussed earlier, keeping pedestal intensity below the target damage threshold is critical to producing a high-density plasma without the complicating effects of a preformed plasma that absorbs and scatters the main pulse. 

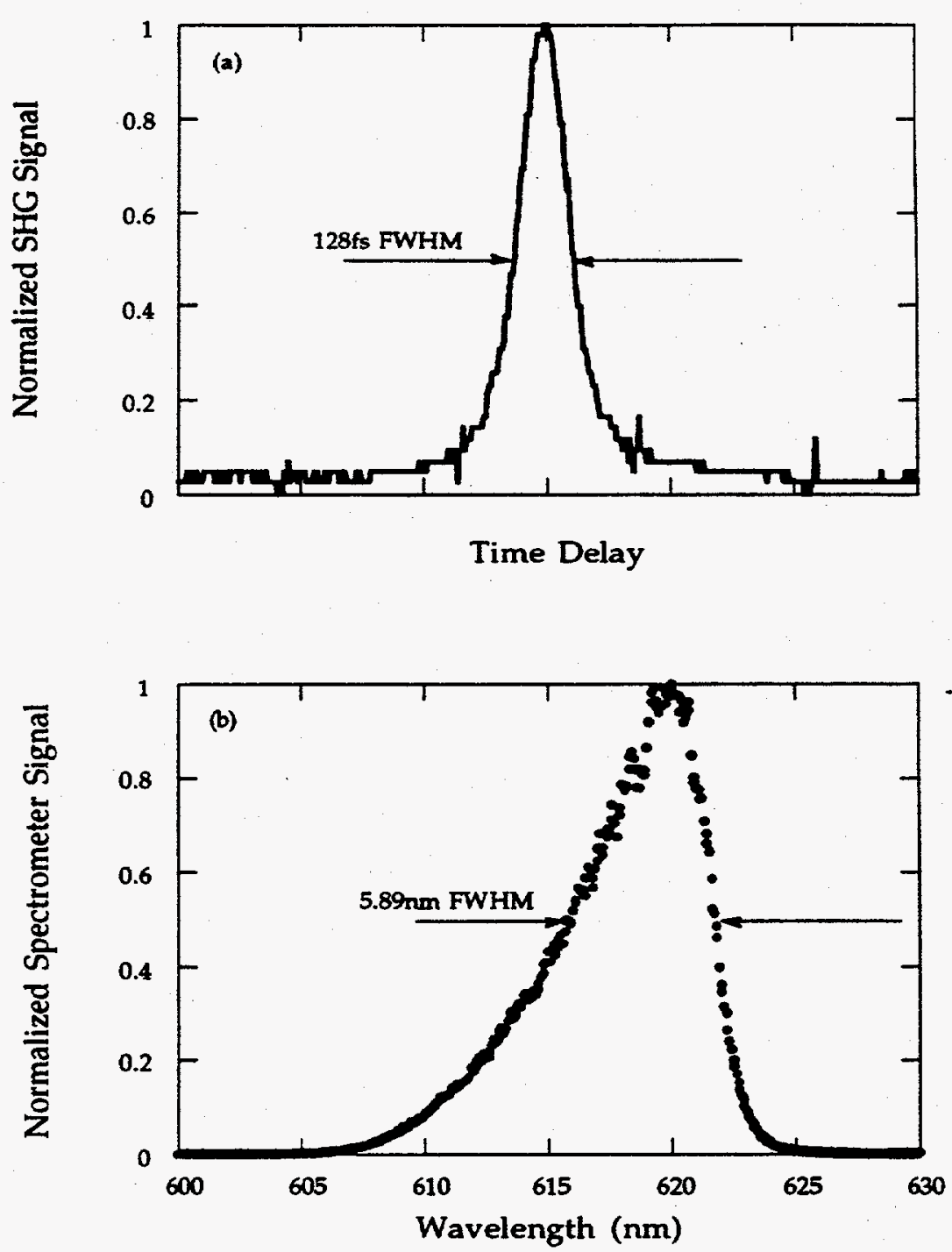

Figure 2.19: The amplified (a) pulse and (b) spectrum.

A contrast ratio measurement involved a series of partial autocorrelation measurements using a scanning autocorrelator (Section 2.6). At the highest intensity, $\sim 1016 \mathrm{~W} / \mathrm{cm}^{2}$, prepulse intensity must be kept below the target damage threshold of about $10^{12} \mathrm{~W} / \mathrm{cm}^{2}$, so the measurement must have a $10^{4}$ dynamic range to assure this contrast is achieved. The pulse wings produce a very small 
SHG signal since their peak intensity is low. Therefore, when just the wings were overlapped, full pulse energy was used and the SHG signal was amplified. As the autocorrelator delay was changed and higher energy pulse portions overlapped, calibrated neutral density (ND) filters were used to attenuate the SHG signal so there was no saturation of either the SHG or the detector. The final signal was obtained by piecing together recorded low and high energy signals with appropriate adjustments to account for signal modification from the amplifier and ND filters. The technique's schematic is shown in Chapter 6 (Figure 6.7) where a method to enhance the contrast ratio was tested. The result (Figure 2.20) shows the contrast ratio to be $>10^{4}$, so preplasma formation was avoided even at the highest intensities. Saturated peaks in Figure 2.20 which begin -1.5 ps from zero delay are back surface reflections from a pellicle beamsplitter in the autocorrelator. This was proven by replacing the beamsplitter with a thicker pellicle that moves the peaks to a greater time delay.

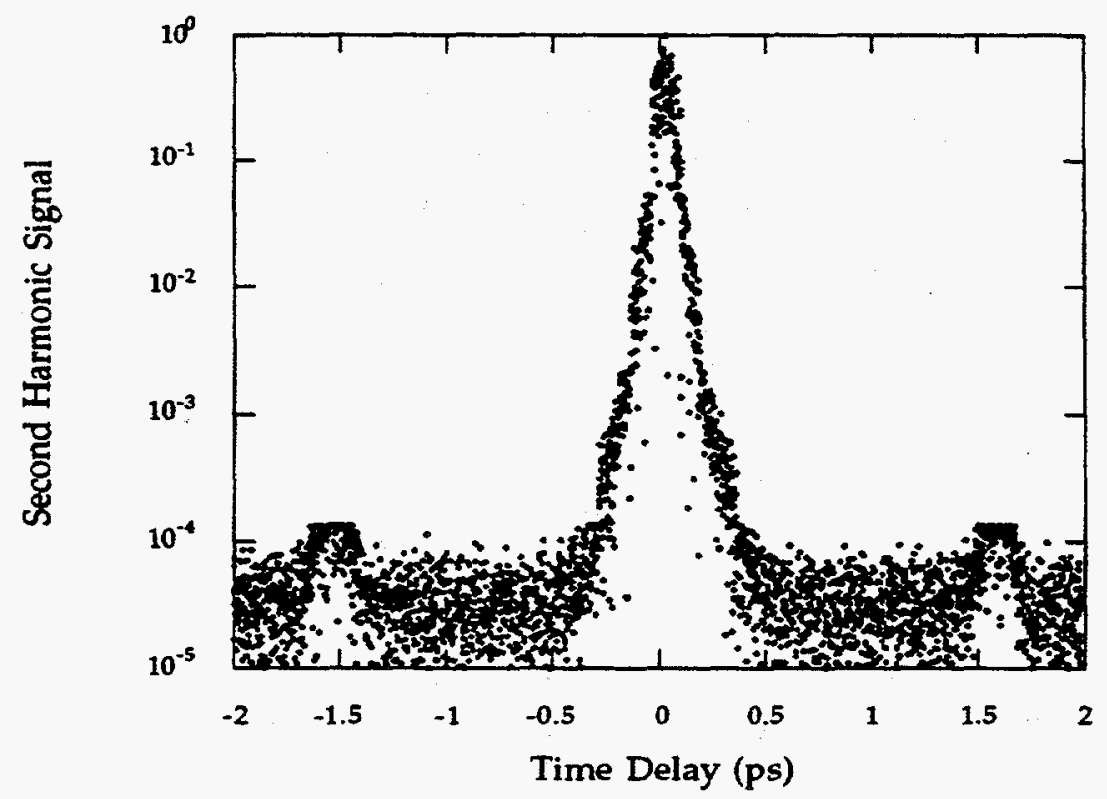

Figure 2.20: High-dynamic-range amplified pulse autocorrelation at peak energy $>3 \mathrm{~mJ}$. 


\subsubsection{The Nd:YAG Amplifier Pump Laser}

A neodymium-doped yttrium aluminum garnet, or Nd:YAG, laser (Quanta Ray model DCR-4) was used to excite dye amplifiers which raised CPM seed pulse energy from $\sim \mathrm{pJ}$ to $\sim \mathrm{mJ}$. The Nd:YAG laser energy levels are shown in Figure 2.21. These levels are modeled by a four level laser transition scheme. Triply ionized neodymium is optically pumped by flashlamps which have an output matching the absorption bands in the red and near IR. Electrons excited to the pump bands rapidly drop to the $F_{3 / 2}$ level which has a long lifetime $(\sim 230 \mu s)$ and acts as the upper laser level. The most likely transition is then to the $I_{11 / 2}$ level $(1064 \mathrm{~nm})$. Electrons in the $I_{11 / 2}$ state quickly relax to the ground state, so the population inversion is always high. Competing transitions all have lower gain and a higher threshold than the $1064 \mathrm{~nm}$ transition. This fact and wavelength selective optics limit the laser output to $1064 \mathrm{~nm}$.

A Q-switch is used to shorten the pulsewidth and raise the peak power. The long-lived upper laser state allows a large population inversion buildup while an electro-optic Q-switch (Pockels cell) holds off oscillation by introducing a large cavity loss. The Q-switch configuration is shown in Figure 2.22. During Q-switched operation, flashlamps excite neodymium ions for about 200us. At the point of maximum population inversion, a fast high-voltage pulse is applied to the Pockels cell that rotates the polarization $45^{\circ}$ changing the cavity to a lowloss configuration. The resulting pulse is $<10$ ns with a power of tens of megawatts. 


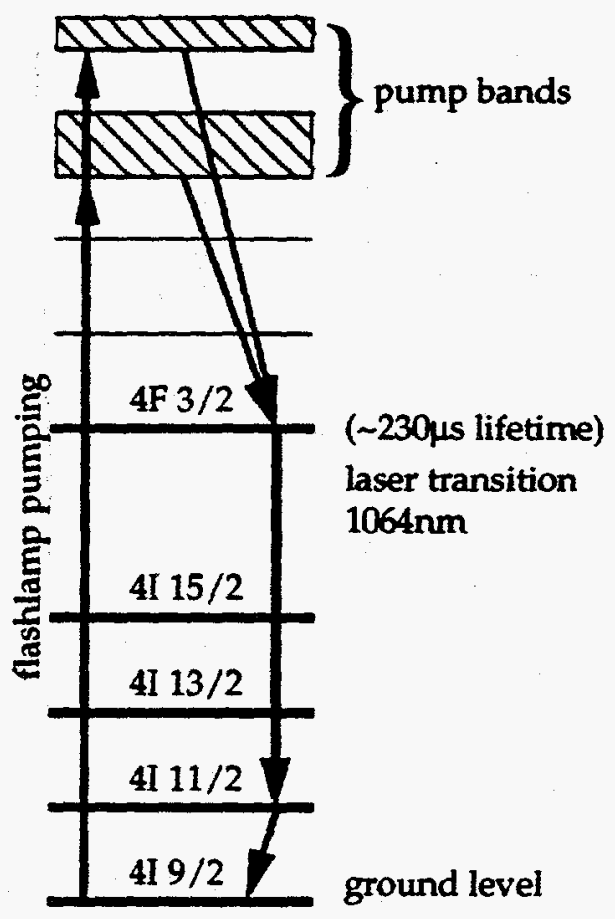

Figure 2.21: Energy leoels for the Nd:YAG pump laser.

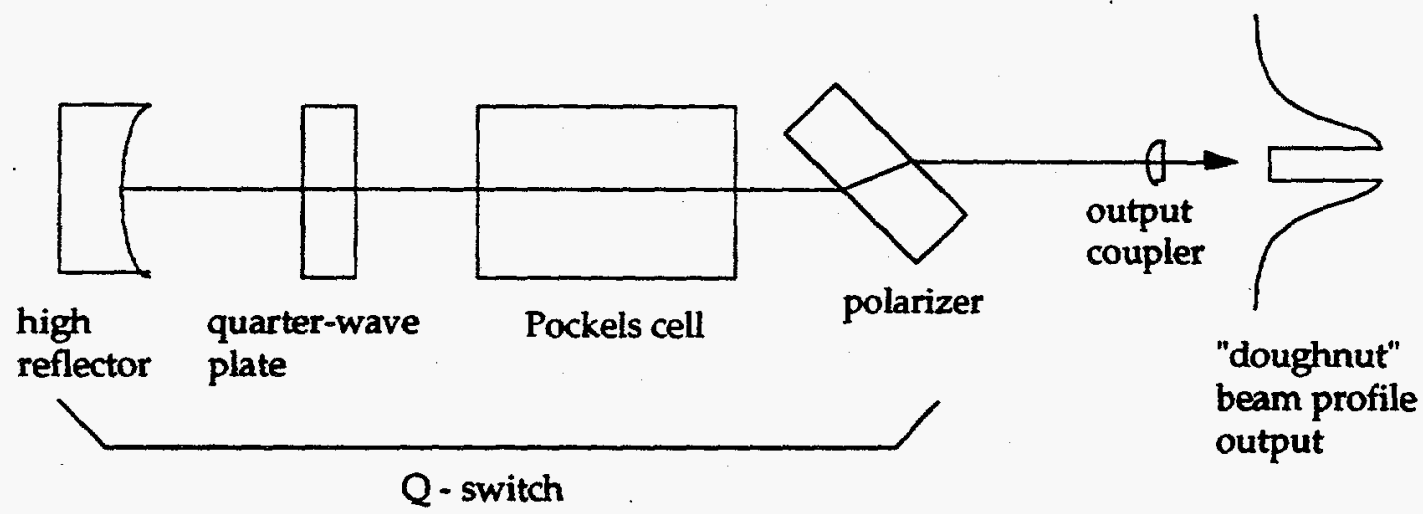

Figure 2.22: The unstable resonator and $Q$-switch of a Nd:YAG laser.

The Nd:YAG laser has an unstable resonator configuration that allows a large intracavity beam diameter and large energy extraction. The beam energy is coupled out around a central mirror at the laser front end. This diffraction coupled resonator (DCR) configuration gives a "doughnut" spatial mode. Uniform laser rod pumping is achieved by placing flashlamps at one focus of a mirrored elliptical pumping chamber while laser rods are at the other focus. 
Thermal lensing in laser rods is compensated by matching the high reflector mirror radius of curvature to the rod focal length. The $1064 \mathrm{~nm}$ output is frequency doubled in a KD*P crystal (type II phasematching) to 532nm to match the dye amplifier absorption.

\subsection{MEASUREMENT OF ULTRA-SHORT PULSES}

How is the temporal width of an ultra-short pulse, which is orders of magnitude shorter than electronic response times, measured? The only temporal scale short enough is the light pulse itself.

An autocorrelator is a device configured in a Michelson interferometer geometry that uses the pulse to measure itself (see Figure 2.23). In a scanning autocorrelator, the pulse is split by a $50 / 50$ beamsplitter and each beam travels down a different arm. Both pulses are focused and overlapped in a nonlinear medium such as a crystal of $\mathrm{KH}_{2} \mathrm{PO}_{4}(\mathrm{KDP})$. The instantaneous nonlinear optical material response generates a signal proportional to the temporal integral of the pulse overlap. This signal is detected by a photomultiplier tube. The total autocorrelation signal is obtained by recording the nonlinear signal generated as a function of the temporal overlap between the two pulses. Overlap is varied by changing the length of one autocorrelator arm, while the other remains fixed. This can be done either in a continuous, rapidly cycling, motion, where the reference mirrors are mounted on a vibrating "shaker" diaphragm (Figure 2.23 (a)), or stepwise, where a translation stage covers the full overlap region pointby-point (Figure 2.23 (b)). 
$1 / 4$ waveplate

(a)

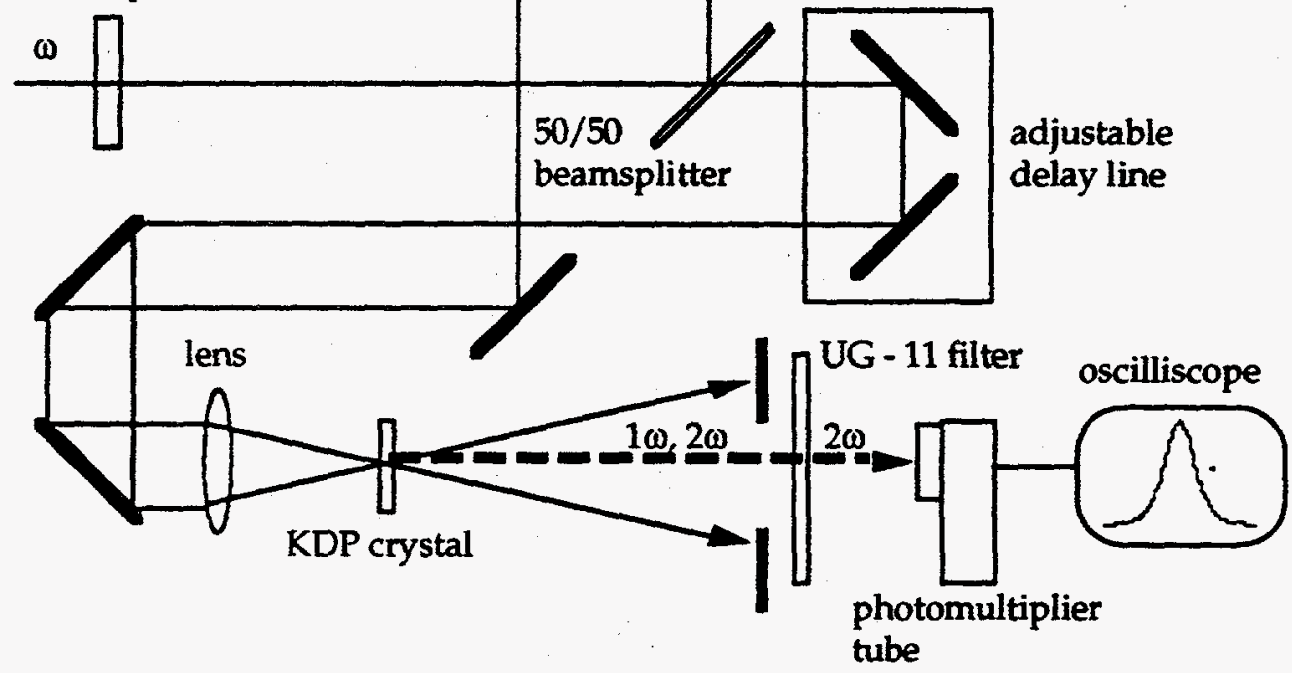

$1 / 4$ waveplate

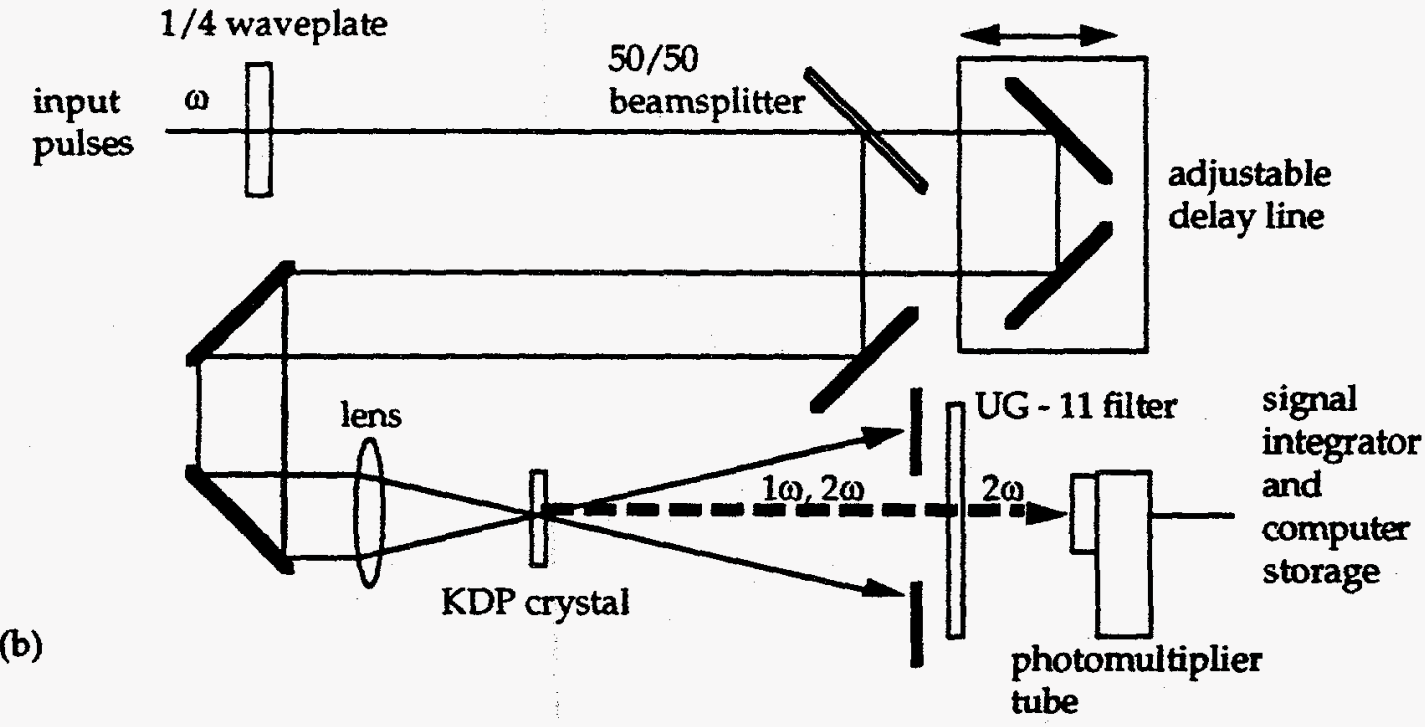

Figure 2.23: (a) The rapid-scan (shaker), second-order autocorrelator. (b) The slow-scan, second-order autocorrelator. 
Analytic formulation of this idea begins with two plane wave pulses of equal amplitude and center frequency $\omega$

$$
E_{1}(\omega)=A_{1}(t) e^{i \omega t} \text { and } E_{2}(\omega)=A_{2}(t) e^{i \omega t}
$$

where $A_{1}(t)$ and $A_{2}(t)$ are the pulse envelopes. If one pulse is delayed in time by changing the length of an autocorrelator arm the total field becomes

$$
E_{1}(\omega, t)+E_{2}(\omega, t+\Delta t)=e^{i \omega t}\left[A_{1}(t)+A_{2}(t+\Delta t) e^{i \omega \omega t}\right]
$$

When this field interacts in a second harmonic generation crystal such as KDP the output second harmonic intensity is

$$
\begin{aligned}
I_{2 \omega}(t) & \sim\left|\left[E_{1}(\omega, t)+E_{2}(\omega, t+\Delta t)\right]^{2}\right|^{2} \\
& =I^{2}(t)+I^{2}(t+\Delta t)+4 I(t) I(t+\Delta t)
\end{aligned}
$$

where $I(t)=|E(t)|^{2}$ and all oscillatory terms are averaged. The photomultiplier detector integrates over time, so the second harmonic signal (normalized to background) is

$$
\begin{aligned}
& I(2 \omega, t) \propto \frac{\langle I(t) I(t+\Delta t)\rangle}{\left\langle I^{2}(t)\right\rangle} \equiv G^{2}(\Delta t) \\
& \langle I(t) I(t+\Delta t)\rangle \equiv \int_{-\infty}^{\infty} I(t) I(t+\Delta t) d t
\end{aligned}
$$

Autocorrelation does not provide a direct pulse shape display, since the signal is proportional to the correlation function, $G^{2}(t)$, and not the intensity itself. To determine the actual pulsewidth, a pulse whose autocorrelation shape fits the detected autocorrelation signal best must be assumed. Consider a Gaussian pulse with FWHM width of $2 \tau_{L} \sqrt{\ln (2)}$

$$
I(t)=e^{-\left(\frac{t}{\tau_{2}}\right)^{2}}
$$

The autocorrelation signal of the Gaussian pulse is 


$$
\begin{aligned}
G^{2}(t) & =\int_{-\infty}^{\infty} \exp \left(-\left(\frac{t}{\tau_{L}}\right)^{2}\right) \exp \left(-\left(\frac{t-\Delta t}{\tau_{L}}\right)^{2}\right) \\
& =\exp \left(-\frac{\Delta t^{2}}{2 \tau_{L}^{2}}\right) \int_{-}^{\infty} \exp \left(-\frac{2 t^{2}}{\tau_{L}^{2}}\right) \partial t \\
& \propto \exp \left(-\frac{\Delta t^{2}}{2 \tau_{L}^{2}}\right)
\end{aligned}
$$

and this signal has a FWHM of

$$
2 \tau_{L} \sqrt{2 \ln (2)}=\sqrt{2}\left(2 \tau_{L} \sqrt{\ln (2)}\right)=\sqrt{2}\left(F W H M_{\text {Gaussian input }}\right)
$$

Similar "deconvolution" factors can be found to determine the true width of other pulse shapes as shown in Table 2.2

\begin{tabular}{ccc}
\hline Pulseshape & $\mathbf{I}(t)$ & FWHM I(t)/FWHM G G $^{2}(t)$ \\
\hline Gaussian & $\exp \left(-4 \ln 2\left(t / \tau_{L}\right)^{2}\right)$ & $\sqrt{2}$ \\
sech $^{2}$ & $\cosh ^{-2}\left(1.76 t / \tau_{L}\right)$ & 1.55 \\
Lorentzian & {$\left[1+\left(2 t / \tau_{L}\right)^{2}\right]^{-1}$} & 2 \\
\hline
\end{tabular}

Table 2.2: Correlation widths for three transform-limited pulse shapes.

To determine which deconvolution factor to use, the measured autocorrelation signal was fit to each theoretical pulseshape as shown in Figure 2.24. The $\operatorname{sech}^{2}$ pulseshape was used.

In general, higher-order correlation functions must be used in addition to $G^{2}(t)$, to uniquely determine the pulseshape [86]. This means a higher order nonlinear process, such as frequency tripling or four-wave-mixing, must be used in the autocorrelator. The $n$th order correlation function is

$$
G^{n}\left(\tau_{1}, \tau_{2}, \ldots \tau_{n-1}\right)=\frac{\left\langle I(t) I\left(t+\tau_{1}\right) \ldots I\left(t+\tau_{n-1}\right)\right\rangle}{\left\langle I^{n}(t)\right\rangle}
$$


It has been shown theoretically $[87,88]$, that an exact knowledge of both $G^{2}$ and $G^{3}$ is sufficient for an exact pulse description. Most significantly for our purposes is that the second order autocorrelation will never show any asymmetry the pulse may possess, because it depends on the square of the intensity. A pulse with a steeply rising edge and slower trailing edge (or vice versa) will appear on a second order correlation to have a rise and fall time somewhere in between. This can have important implications in determining how much energy is absorbed in a plasma before a reflective layer forms during the pulsewidth.

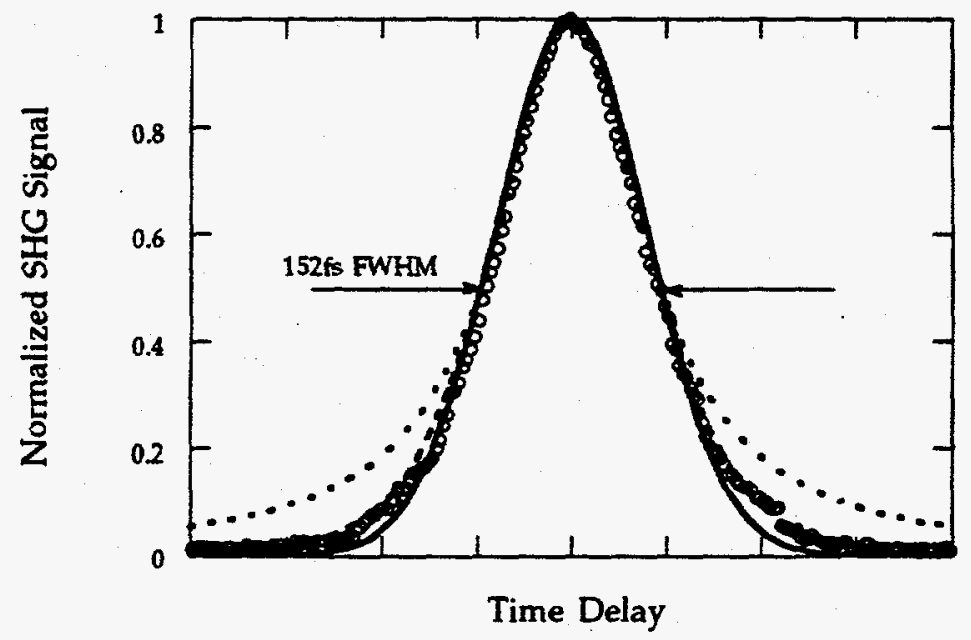

Figure 2.24: Pulse shape compared with fits to Gaussian (solid), $\operatorname{sech}^{2}$ (dash) and Lorentzian (dot) curoes.

Many methods exist for obtaining the full autocorrelation trace with a single laser pulse rather than over a series of pulses at different time delays [8993]. The "single-shot" autocorrelator used in these experiments is shown in Figure 2.25 [93]. The same principles of scanning autocorrelators apply to a single-shot autocorrelator, except that pulses are not focused in the nonlinear medium (or are focused with a cylindrical lens) and both autocorrelator arms are fixed. The temporal pulse shape is transformed into a spatial shape that can be 
detected by a linear diode array and analyzed in real time on an oscilloscope. All autocorrelator information obtained by scanning the pulse across itself in time is obtained here with a single pulse in the following manner. Figure 2.25 shows that at one point within the crystal pulse 1's leading edge interacts with pulse 2's trailing edge. At the opposite end of the interaction region, pulse 1's trailing edge interacts with pulse 2's leading edge. Along the line between these points in the crystal all other pulse overlap combinations occur. The information along this line is the same as scanning one pulse completely across the other in time by adjusting the path length of one autocorrelator arm. The temporal delay is related to the spatial position the diode array measures by [93]

$$
\tau=\frac{n x_{0} \sin (\phi / 2)}{c}
$$

where $\phi$ is the angle between the two incident beams, $n$ is the nonlinear crystal index, $c$ is the speed of light, and $x_{0}$ is the transverse position.

Autocorrelators are calibrated in the following manner. An autocorrelation trace with the pulses perfectly overlapped in time is recorded. The pathlength for one autocorrelator arm is changed by a known amount. This change shifts the position where complete temporal overlap occurs so the pulse shifts on the oscilloscope time axis. The time difference between the shifted and unshifted pulse on the oscilloscope reading the diode array is correlated to the time difference equal to half the pathlength change divided by the speed of light. The pulse FWHM is then measured and divided by the appropriate deconvolution factor for the assumed pulseshape as in Table 2.2. 


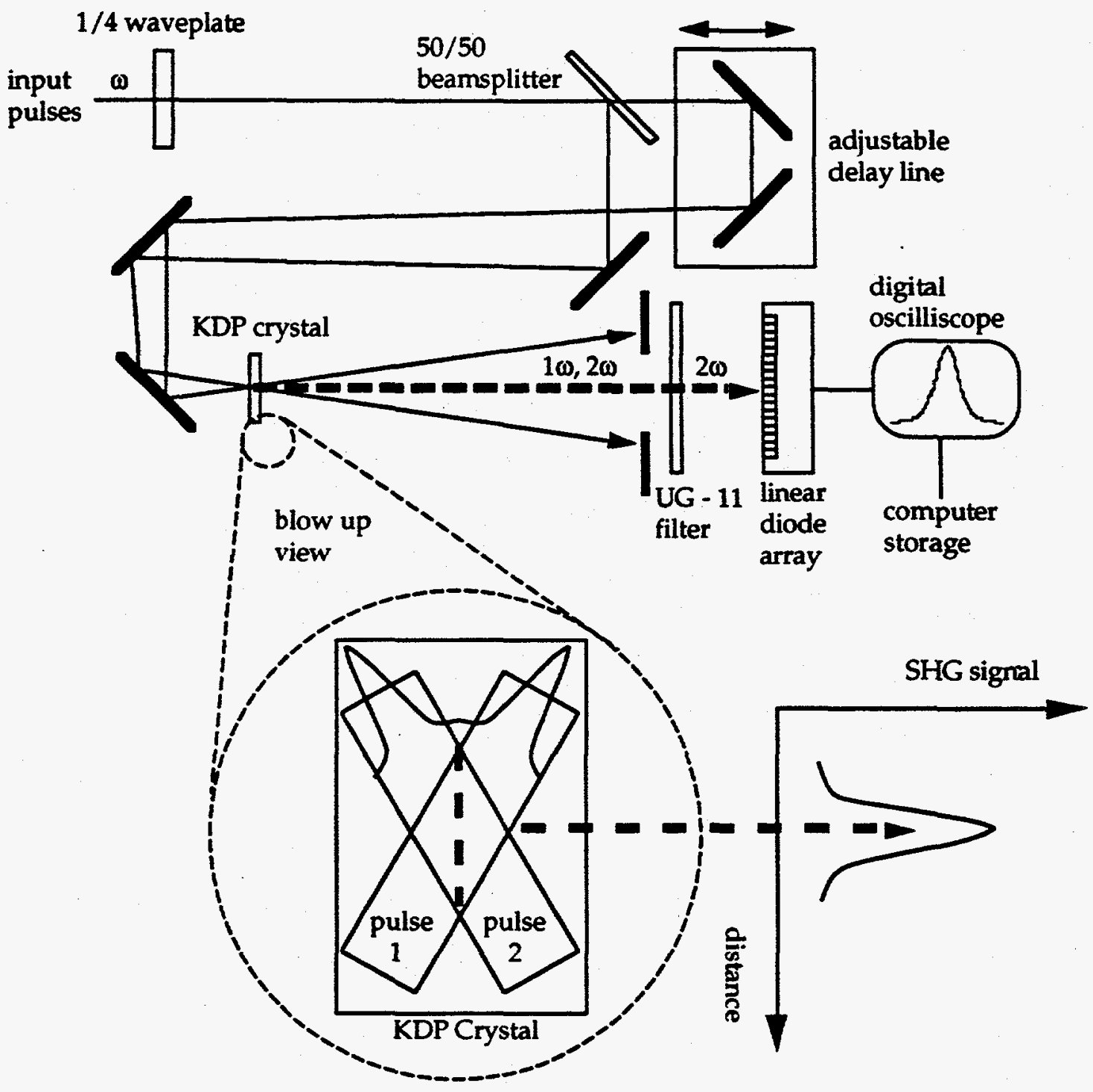

Figure 2.25: The single-shot, second-order autocorrelator. 


\section{CHAPTER 3:}

\section{AN INTRODUCTION TO LASER-PRODUCED PLASMAS}

\subsection{INTRODUCTION}

Three separate, but interrelated, physics issues arise in this experiment: (1) dense plasma physics, (2) atomic ionization physics with strong fields, and (3) electromagnetic field propagation in a plasma. A complete understanding of the radiation-matter interaction involves all of these areas in a self-consistent picture. This, of course, is a daunting task, so the simplest approximation is to treat each physical process as independent. Ionization physics is treated as an atomic physics problem without complication from dense plasma effects. Similarly, field propagation is not modified by ionization during propagation and so on. Within each separate problem, further approximations are made to obtain tractable solutions and physical insight. An introduction to basic issues in each area is treated here with many details left to references given.

\subsection{THE LASER PLASMA: LONG PULSE AND SHORT PULSE PLASMAS}

Fundamental differences exist between a plasma created by a pulse longer than about one picosecond and a plasma created by a subpicosecond pulse. Plasma conditions also depend on target material, laser wavelength, and laser intensity, but the most striking differences occur between the physics of long (>1ps) and short (<1ps) pulse plasmas.

\subsubsection{Long Pulse Plasmas}

When a long laser pulse is used to create a plasma from a solid target, a low electron density plasma forms on the target surface during the pulse's 
leading edge. The plasma expands during heating by the pulse at about the sound speed, $c_{s}$ [94]

$$
v=c_{s} \approx \sqrt{Z T_{e} / M_{i}} \sim 10^{7}-10^{8} \mathrm{~cm} / \mathrm{s}
$$

where $Z$ is the average ion stage, $M_{i}$ is the ion mass, and $T_{e}$ is the electron temperature. Although electrons are much faster $\left(v_{e} \approx \sqrt{T_{e} / m_{e}}\right)$, heavy ions are electrically coupled to electrons to maintain charge neutrality, so resulting ambipolar diffusion is much slower. At $\sigma \approx 10^{7} \mathrm{~cm} / \mathrm{s}$, the plasma will expand about $0.1 \mu \mathrm{m} / \mathrm{ps}$ which is a significant fraction of a wavelength for visible wavelength lasers with a pulsewidth $>1$ ps. Expanding plasma during the pulse's leading edge modifies the remainder of the pulse propagation [95]. The dispersion relation for the light traveling within the plasma is [96]

$$
\omega_{l}^{2}=\omega_{p}^{2}+c^{2} k^{2}
$$

where $k$ and $\omega_{l}$ are the laser wavenumber and frequency, $c$ is the speed of light, and $\omega_{p}$ is the plasma frequency

$$
\omega_{p}^{2}=\frac{4 \pi n_{e} e^{2}}{m_{e}}
$$

where $n_{e}, m_{e}$, and $e$ are the electron density, mass, and charge respectively. The plasma frequency describes the natural frequency of electron electrostatic oscillation resulting from a disturbance of charge neutrality. That is, $1 / \omega_{p}$ is the characteristic response time for electron motion. When a light wave is incident, electrons respond instantaneously and reradiate light back if $\omega_{1}<\omega_{p}$ (note $k$ is imaginary when $\left.\omega_{l}<\omega_{p}\right)$. Thus, the condition $\omega_{l}=\omega_{p}$ defines the maximum plasma density to which a light wave can propagate. This maximum, or critical, density $n_{c}$ is

$$
n_{c}=\frac{m_{e} \omega_{1}^{2}}{4 \pi e^{2}}=1.1 \times 10^{21}\left(\frac{1 \mu m}{\lambda_{1}}\right)^{2} \mathrm{~cm}^{-3}
$$

Beyond this density, the light wave is exponentially attenuated and will penetrate only on the order of a skin depth. As mentioned in Section 2.1, the skin 
depth is distance in which the electromagnetic wave is damped to $1 / e$ of its amplitude. The lower density region before critical density is termed the blowoff, underdense, or coronal region. Critical densities that most lasers can reach in a plasma are shown in Figure 3.1.

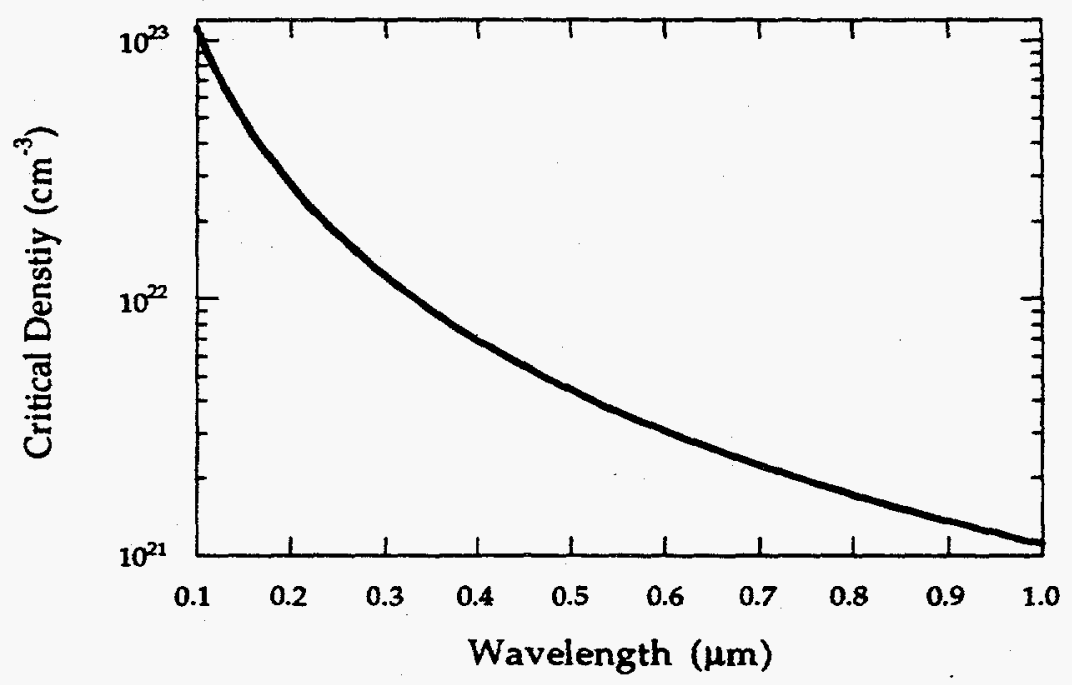

Figure 3.1: The critical density versus wavelength for most laser wavelengths.

A visible or longer wavelength laser $(>0.4 \mu \mathrm{m})$ is only able to interact with a plasma density of $<10^{22} \mathrm{~cm}^{-3}$. Thus, the only way the pulse can interact with a plasma at or above solid density $\left(-10^{23} \mathrm{~cm}^{-3}\right)$ is to limit supercritical coronal region expansion to a small fraction of a skin depth, $\delta$, while the pulse is present. A typical skin depth for subpicosecond pulses is

$$
\delta=\sqrt{c^{2} / 2 \pi \omega_{l} \sigma} \sim \text { several hundred } \AA
$$

where $\sigma$ is the plasma conductivity. This is a key difference between short and long pulse interactions. An ultra-short pulse of $\sim 100 \mathrm{fs}$ will see an expansion of only about $100 \AA$, by the end of the pulse. The evanescent wave penetrating the critical density region interacts directly with the solid density region. 
The coronal region also changes the interaction by absorbing and scattering laser light. As the laser propagates toward critical density a number of mechanisms, such as inverse Bremsstrahlung absorption, resonance absorption, Raman and Brillouin scattering, filamentation, and hot electron generation occur in various underdense plasma regions, as shown in Figure $3.2[94,96]$. Absorption and scattering processes are very efficient $(-90 \%)$, because the underdense region is large $(\sim 1 \mu \mathrm{m})$ and the interaction time is long (>ps).

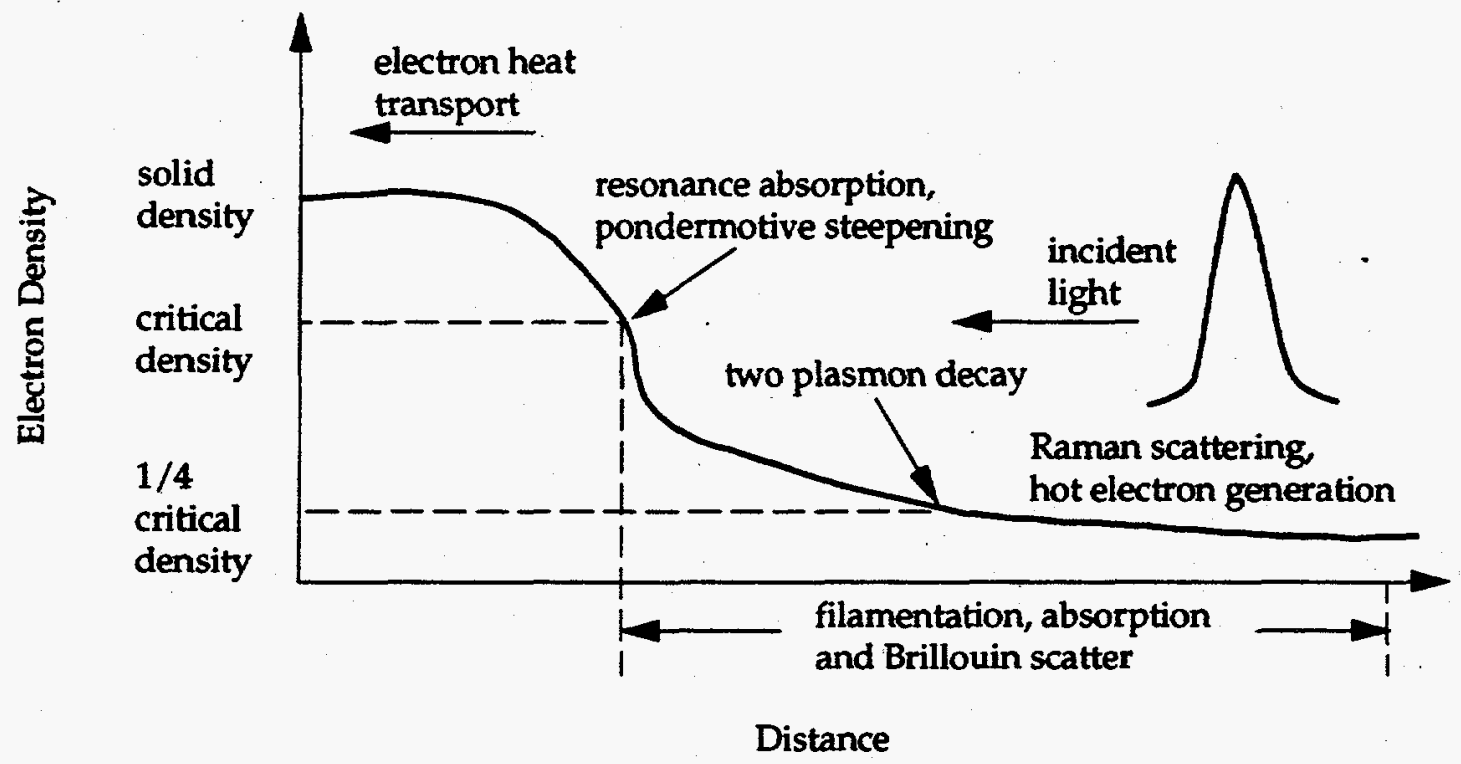

Figure 3.2: Schematic electron density profile in a long pulse (>1ps) laser-produced plasma, showing the location of major light-plasma coupling processes (after [94]).

The underdense region of a long pulse plasma has relatively small temperature and density gradients. The classical heat flux, $\mathbf{q}$, in a plasma can be written

$$
\begin{aligned}
& \mathbf{q}=-\kappa \nabla T \text { ergs } / \mathrm{cm}^{2} s \\
& \kappa \nabla T \approx\left(\frac{\lambda_{\text {mop }}}{L_{T}}\right)(n T) v_{t}, v_{t} \equiv \sqrt{T / M}
\end{aligned}
$$

where $x$ is the heat transfer coefficient, $T$ is the temperature, $n$ is the density, $\lambda_{m f p}$ is the mean-free-path, $L_{T}=T /|d T / d x|$ is the temperature gradient scale 
length, and $v_{t}$ is the thermal velocity. Because electron thermal conductivity is greater than ion conduction by a factor of $\sqrt{M_{i} / m_{e}}$, it is the random walk of electrons that carries heat from hot plasma regions to colder bulk regions. The heat flux equation is then usually written using only electrons

$$
\mathrm{q}_{e}=-\kappa \nabla T_{e} \text { ergs } / \mathrm{cm}^{2} s
$$

Since this heat flux is proportional to the relatively small temperature gradient in the underdense region, heat diffusion will be slow. Similarly, plasma hydrodynamic expansion is related to the small density gradient in the corona. Cooling mechanisms in the plasma such as heat diffusion and hydrodynamic expansion are relatively slow due to the small temperature and density gradients. As a result, the $x$-ray emission is found to have a relatively long (>ps) decay time [6].

Long pulse plasmas in dense targets develop slowly in a collisionally, rather than a radiatively, dominated environment. There is enough time for electron collisions to bring the plasma into a state of local thermodynamic equilibrium (LTE) [97]. LTE is the collision-dominated limit of plasma equilibrium states. In complete thermodynamic equilibrium, all atomic and atom-radiation interaction processes exist in detailed balance. For example, the collisional ionization rate and three-body recombination rate are in balance, $S_{Z-1}(p)+e \leftrightarrow S_{Z}+e+e$, where $S_{Z}$ is the bare nucleus with charge state $Z$ and $e$ is the electron. In this case, the distribution between ionization stages is given by the Saha equation, the electronic population amongst bound levels is governed by the Maxwell-Boltzmann distribution, and the free electron velocity distribution is Maxwellian - all distributions being characterized by the same temperature $T$. In LTE, radiative processes become negligible compared with collisional ones and a detailed balance with the distributions mentioned above exists from collisional processes alone. 


\subsubsection{Short Pulse Plasmas}

A subpicosecond pulse produces remarkably different plasma conditions than longer pulses. Expansion is limited to less than an optical skin depth during the pulse's leading edge. Light is absorbed in solid density material surface layers. Long scale length absorption and scattering mechanisms present in long pulse plasmas do not occur, since there is no large coronal region. Reflection from the dense, ionized skin depth is very efficient, $-70 \%$ for insulator, semiconductor and metal targets at $1016 \mathrm{~W} / \mathrm{cm}^{2}$ using S-polarization. This value is determined from this work as presented in Chapter 4. Absorption occurs mainly in the ionized solid by linear and nonlinear inverse Bremsstrahlung for intensities $<10^{16} \mathrm{~W} / \mathrm{cm}^{2}[6]$ and by resonance absorption and vacuum heating for P-polarized light [96]. Absorbed energy is deposited only in a thin optical skin depth. This leads to very large initial temperature and density gradients that rapidly transfer heat, by electron conduction and hydrodynamic expansion, to regions outside the skin depth. The plasma rapidly cools and recombination and radiation (including $\mathrm{keV} x$-ray radiation), occur on a subpicosecond timescale [6].

Enormous electric fields in intense, ultra-short pulses can drive a variety of nonlinear processes [98]. These include modification of the electron-ion collision trajectory and electron distribution function [99-105], and density profile steepening. The electron-ion collision rate decreases because the electron trajectory is modified by the oscillatory motion in a strong laser field [101]. The electron distribution function shifts and skews from the thermal Maxwellian found in long pulse plasmas towards higher energies in strong fields [100]. Both of these high-field modifications are discussed further in Section 4.3.3.2. The critical parameter for these nonlinear effects is the ratio of electron quiver velocity in the electric field, $v_{\text {osc }}=e E / m \omega$, to electron thermal velocity, $v_{t h}=\left(T_{e} / m\right)^{1 / 2}$ 


$$
\frac{v_{o s c}}{v_{t h}} \approx\left(\frac{I}{10^{16} \mathrm{~W} / \mathrm{cm}^{2}}\right)^{1 / 2}\left(\frac{\lambda}{249 \mathrm{~nm}}\right)\left(\frac{200 \mathrm{eV}}{T_{e}}\right)^{1 / 2}
$$

When the intensity is such that $v_{o s c}>v_{t k}$, these nonlinear effects become important.

The main ionization mechanism in plasma formation changes from collisional avalanche ionization present in long pulse plasmas to multi-photon or tunneling ionization. During the pulse's rising edge, these field ionization mechanisms act much faster than ion states can collisionally equilibrate, so the plasma can form in a non-LTE (NLTE) state. This NLTE state is a transient driven by the laser field. It rapidly relaxes to LTE once the field is gone and collisions in the dense plasma dominate. Finally, intense light field pondermotive pressure can push and distort the electron density profile. These nonlinearities are addressed in later sections and in Chapter 4. Differences between long and short pulse plasmas are summarized in Table 3.1.

\begin{tabular}{ll}
\hline \multicolumn{1}{c}{ Long Pulse Plasma } & \multicolumn{1}{c}{ Short Pulse Plasma } \\
\hline coronal region & no coronal region \\
scattering and absorption in corona & absorption in solid density \\
efficient absorption & efficient reflection \\
small $\mathrm{T}_{\mathrm{e}}$ and $\mathrm{n}_{\mathrm{e}}$ gradients & large $\mathrm{T}_{\mathrm{e}}$ and $\mathrm{n}_{\mathrm{e}}$ gradients \\
slow cooling & rapid cooling \\
long radiation pulse emission & short radiation pulse emission \\
linear laser-plasma coupling & some nonlinear laser-plasma coupling \\
LTE formation & transient non-LTE formation \\
\hline \hline
\end{tabular}

Table 3.1: Comparison of general plasma characteristics created by long (>1ps) and short (<1ps) laser pulses. 


\subsection{ATOMIC IONIZATION}

The strong electric field in short-pulse plasmas introduces new mechanisms for atomic ionization in plasmas. Conventional laser plasmas are produced with relatively low peak intensities. The dominant mechanism for breakdown in solids by irradiation with lower intensity and longer pulse lasers is linear inverse Bremsstrahlung followed by electron-ion collisional ionization [14, 15]. Amplified, ultra-short pulses, however, can be focused to peak intensities that correspond to electric fields on the order of or above atomic fields $\left(3.5 \times 10^{16} \mathrm{~W} / \mathrm{cm}^{2}=1 \mathrm{a} . \mathrm{u}\right.$.). At these fields, the laser can ionize atoms directly with multi-photon ionization (MPI) and tunneling ionization $[8,9]$.

The rising edge of an intense, ultra-short pulse plasma initially forms a dense collection of field-ionized ions with a relatively cold electron population. This transient state disappears as inverse Bremsstrahlung rapidly increases the electron temperature and collisional ionization becomes important because the plasma density is high following field ionization. The importance of fieldionization to plasma formation is discussed in detail in Chapter 4.

\subsubsection{Ionization in Gases}

\subsubsection{Multi-photon and Tunneling Ionization}

Rigorous field-induced atomic ionization to produce a plasma is treated with the time-dependent Schrödinger equation

$$
i \hbar \frac{\partial \phi(\mathbf{r}, t)}{\partial t}=H \phi(\mathbf{r}, t)
$$

$H$ is the atom-field system Hamiltonian and may be written as

$$
H=H_{\text {rad }}+H_{\text {atom }}+H_{\text {int }}
$$

where $H_{\text {rad }}, H_{\text {atom }}$, and $H_{\text {int }}$ are Hamiltonians of the radiation field, the atom, and the atom-field interaction. The interaction Hamiltonian includes terms for potential and kinetic energies, absorption, emission, and scattering, as well as 
other corrections from the Zeeman effect, the spin-orbit interactions, and relativistic energy effects. The physical ionization picture depends on which method is used to solve the time-dependent Schrödinger equation. When perturbation theory can be used, and in the high frequency limit, ionization is thought of in a multi-photon ionization picture. At higher intensities and lower frequencies, perturbation theory begins to fail and an electron tunneling picture is used with a classical electric field.

Multi-photon ionization is pictured as shown in Figure 3.3. An atom absorbs a number of photons simultaneously which brings an electron to continuum states. The absorption process occurs through laser-induced virtual states, which are not atomic eigenstates. In theory, MPI does not require any atomic state between the initial state and final (continuum) state. Laser-induced virtual states, which are associated with the photon energy and harmonics, act as real atomic states. Virtual state lifetimes are much shorter than normal atomic state lifetimes. The atom is within the laser field for a time on the order of one optical cycle, $-10^{-15} \mathrm{~s}$. Multiple photon absorption through all virtual states to the continuum (Figure 3.3 (a)) must occur in a time shorter than $\sim 10^{-15} \mathrm{~s}$. Only the large photon flux achievable with intense lasers is enough to cause this multiphoton process. However, the usual case is that a real atomic state is located near a laser-induced virtual state (Figure 3.3 (b)). In this case, the time required for multiple photons to act together is increased and determined by $1 / \mathrm{dE}$, where $\mathrm{dE}$ is the energy defect. This quasi-resonant process requires a lower laser intensity.

Tunnel ionization can be viewed as a classical electric field from the laser pulse suppressing the atomic potential, as shown in Figure 3.4. The electron then has a finite probability of tunneling through the suppressed atomic potential barrier and escaping the atom. Note that the electron is treated as a wave or it 
could not possibly pass over the barrier in the classical picture. This semiclassical picture generally replaces the quantum picture of multi-photon processes (MPI) as the intensity, i.e., the number of photons, becomes so great that the field can be treated classically.

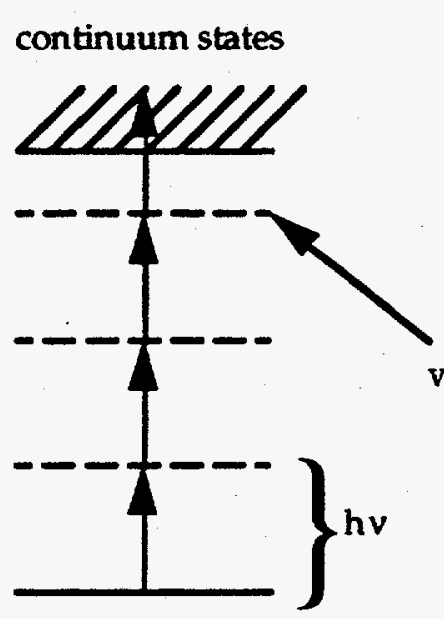

(a) ground state continuum states

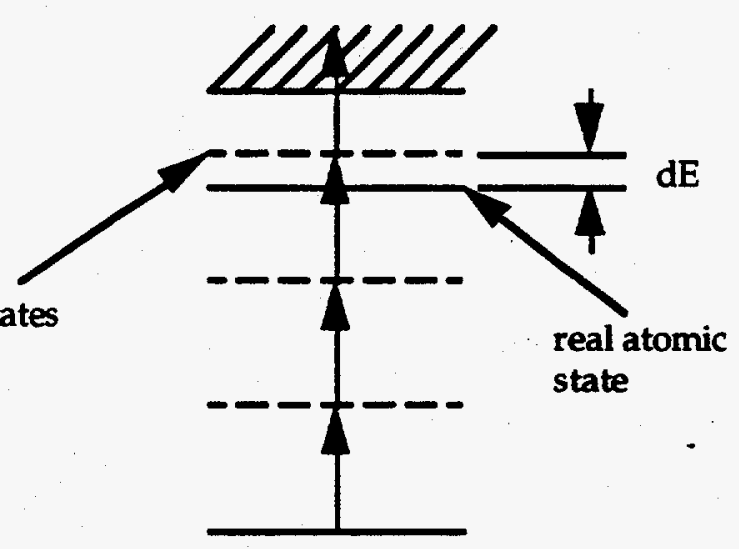

(b) ground state

Figure 3.3: MPI (4 photon) picture of ionization of an atom: (a) non-resonant process using only virtual states. (b) quasi-resonant process where $d E$ is the energy defect between the energy of three photons and the energy of the closest allowed atomic state (after [8]).

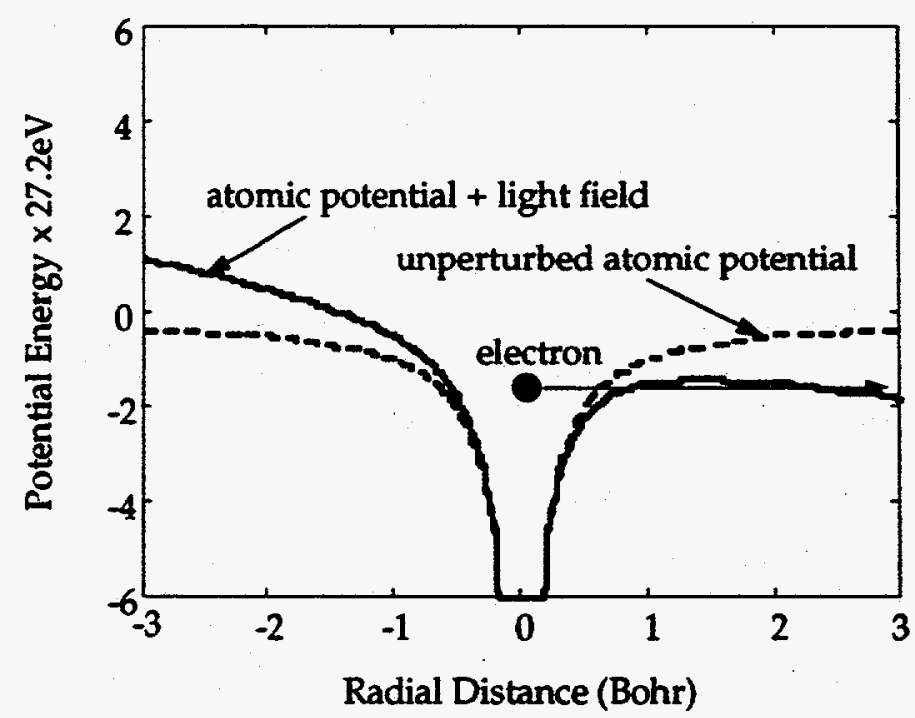

Figure 3.4: Atomic potential distortion by a strong light field allows tunneling ionization to occur. 
The MPI and tunneling pictures are related in the following manner [10]: The tunneling time, $\tau$, for an electron of mean velocity $v_{t} \sim \sqrt{I / m}$ to pass through a barrier of width $l=I / e E$, where $I$ is the atomic ionization potential and $E$ is the electric field intensity, is approximately $\tau=l / v_{t}$. For laser frequencies up to the order of the tunnel frequency $\omega_{t}=e E / \sqrt{2 m I}$, the field will appear static and tunneling ionization will be determined solely by the instantaneous field intensity value. At frequencies higher than $\omega_{t}$, the electron does not have time to tunnel through the barrier before the laser cycle changes the barrier potential. It is expected that the ionization probability will contain a frequency dependence as in MPI. Keldysh [10] derives a single formula that shows the MPI and tunneling ionization to be the same. At low frequencies $\left(\omega<<\omega_{t}\right)$ and very strong fields, tunneling ionization is appropriate while at higher frequencies $\left(\omega>>\omega_{t}\right)$ and weaker fields MPI is appropriate.

The ionization probability is obtained [10] by looking at the probability of transition from the ground state electron wavefunction

$$
\psi_{0}(\mathbf{r}, t)=\psi_{0}(\mathbf{r}) \exp \left\{\frac{i}{\hbar}\left(I_{0} t-\frac{e F \sigma_{0}}{\omega} \sin \omega t\right)\right\}
$$

to the wavefunction of a free electron in an electric field

$$
\psi_{p}(r, t)=\exp \left\{\frac{i}{\hbar}\left[\left(p+\frac{e F}{\omega} \sin \omega t\right) \mathbf{r}-\int_{0}^{t} \frac{1}{2 m}\left(p+\frac{e F}{\omega} \sin \omega \tau\right)^{2} d \tau\right]\right\}
$$

where $F$ is the electric field intensity, $I_{0}$ is the ionization potential of the ground level, $\sigma_{0}$ describes the Stark shift of the ground level, $p$ is the electron momentum, and $\mathbf{r}$ is the electron position. The wavefunctions are written out explicitly because later they are compared to the solution for solid ionization. The general formula for the probability of non-resonant ionization from the ground state is [10] 


$$
\begin{gathered}
w=A \omega\left(\frac{I_{0}}{\hbar \omega}\right)^{3 / 2}\left(\frac{\gamma}{\left(1+\gamma^{2}\right)^{1 / 2}}\right)^{5 / 2} S\left(\gamma, \frac{\tilde{I}}{\hbar \omega}\right) \\
\times \exp \left\{-\frac{2 \tilde{I}_{0}}{\hbar \omega}\left[\sinh ^{-1} \gamma-\gamma \frac{\left(1+\gamma^{2}\right)^{1 / 2}}{1+2 \gamma^{2}}\right]\right\} \\
\gamma=\omega / \omega_{t}=\omega\left(2 m I_{0}\right)^{1 / 2} / e F
\end{gathered}
$$

where $A$ is a numerical coefficient of order unity, the effective ionization potential is defined by $\tilde{I}_{0}=I_{0}+e^{2} F^{2} / 4 m \omega^{2}=I_{0}\left(1+1 / 2 \gamma^{2}\right)$, and $S\left(\gamma, \tilde{I}_{0} / \hbar \omega\right)$ is a slowly varying function of the field frequency defined by

$$
\begin{aligned}
S(\gamma, x) & =\sum_{n=0}^{\infty} \exp \left\{-2[\langle x+1\rangle-x+n]\left[\sinh ^{-1} \gamma-\frac{\gamma}{\sqrt{1+\gamma^{2}}}\right)\right\} \\
& \times \Phi\left\{\left[\frac{2 \gamma}{\sqrt{1+\gamma^{2}}}(\langle x+1\rangle-x+n)\right]^{1 / 2}\right\}
\end{aligned}
$$

where $\langle x\rangle$ denotes the integer part of $x$ and the function $\Phi(z)$ is

$$
\Phi(z)=\int_{0} e^{y^{2}-z^{2}} d y
$$

A similar solution can be found [10] for the ionization probability when higher incident field harmonics are close to a resonance with the electronic transition. The atom is excited and then ionized from the excited state in this situation.

The parameter $\gamma=\omega / \omega_{t}=\omega\left(2 m I_{0}\right)^{1 / 2} / e F$ in Eq. (3.14) is the conventional boundary dividing the MPI and tunneling regimes. When the ionization time is greater than an optical cycle $(\gamma>1), \mathrm{MPI}$ is a good ionization picture, but when the atom ionizes in less than an optical cycle $(\gamma<<1)$, tunneling is a more appropriate picture. When $\gamma<<1$ and $\omega \rightarrow 0$, the solution, Eq. (3.13), becomes

$$
w_{0}=\frac{\sqrt{6 \pi}}{4} \frac{I_{0}}{\hbar}\left(\frac{e F \hbar}{m^{1 / 2} I_{0}^{3 / 2}}\right)^{1 / 2} \exp \left\{-\frac{4}{3} \frac{\sqrt{2 m} I_{0}^{3 / 2}}{e \hbar F}\right\}
$$

which matches that of tunnel ionization through a barrier [106]. Note that the solution does not depend on the frequency, only the instantaneous field. It is a 
static picture. In the opposite case, when $\gg>1$ and $\omega$ is high, Eq. (3.13) reduces to the probability of simultaneous absorption of several photons

$$
\begin{aligned}
w_{0}= & A \omega\left(\frac{I_{0}}{\hbar \omega}\right) \exp \left\{2\left(\frac{\tilde{I}_{0}}{\hbar \omega}+1\right)-\frac{\tilde{I}_{0}}{\hbar \omega}\left(1+\frac{e^{2} F^{2}}{2 m \omega^{2} I_{0}}\right)\right\} \\
& \times\left(\frac{e^{2} F^{2}}{8 m \omega^{2} I_{0}}\right)^{I_{0} \hbar \omega+1} \Phi\left[\left(2\left(\frac{\tilde{I}_{0}}{\hbar \omega}+1\right)-\frac{2 \tilde{I}_{0}}{\hbar \omega}\right)^{1 / 2}\right]
\end{aligned}
$$

Eqs. (3.15) and (3.16) describe the ionization of hydrogen, but accounting for the correct Coulomb interaction in the final state makes the solution applicable to any atom and only changes the pre-exponential factor not the solution form.

\subsubsection{Other Ionization Models}

Keldysh's "simple atom" tunneling and the MPI models were the first in what is now a large number of models describing atomic ionization by multiphoton processes. Tunneling is the only model referenced here, because it describes the essential features of ionization. Details in more advanced ionization theories are less important than using the proper laser-plasma interaction model. For completeness, theoretical work treating multi-photon processes is outlined here and references are found in Gavrila [9]. In the 1960's and 1970's, the first laser ionization experiments had relatively small fields compared to the atomic field. Lowest order time-dependent perturbation theory (LOPT) was generally capable of describing non-resonant MPI processes. Theoretical treatments of hydrogenic atoms were developed first. Resonant MPI processes used an effective Hamiltonian description. In addition, the role of autoionizing states in MPI was investigated. More advanced methods than LOPT were developed in parallel to include: summation of the perturbation series, the Floquet theory, and reformulation of the Schrödinger equation using space translation and analytic models. The Keldysh theory was also improved 
by S-matrix theory to give the so-called KFR theory. The Keldysh theory is considered an adiabatic approximation valid for $\omega<<\omega_{t}$. It predicts the correct exponential probability dependence of ionization on the field amplitude, but the coefficients are not complete [107]. To obtain a correct coefficient the exact quasiclassical electron wave function must be known which takes into account the external field effect and the electron interaction with the remaining ion. The theory is generalized to include these effects in a hydrogen atom by treating the case of an alternating electric field. Finally, in these early works only expressions for the tunnel ionization probability from arbitrary state of the hydrogen atom were derived. In 1986, Ammosov [108] derived an equation for the tunnel ionization probability of complex atoms or atomic ions in arbitrary states. This so-called ADK model treats complex atom states with effective principle and orbital quantum numbers that account for quantum effects and the degree of ionization in an ion.

\subsubsection{Ionization in Solids}

Keldysh has shown $[10,109]$ that the asymptotic, i.e., $\gamma>1$ and $\gamma<<1$, ionization probability behavior in a solid is similar to that of gases for both high and low frequencies. This is important to this experiment, since it allows the use of a simple ionization model [110] developed previously for gas ionization experiments [111].

The essential difference in solid ionization is the ionization process involves electron transfer from the valence to the conduction band and an electron-hole pair is created. The final state energy, $\varepsilon(\mathbf{p})$, is no longer that of a free electron, it now involves the sum of the electron and hole energies, $\varepsilon(\mathbf{p})=\varepsilon_{c}(\mathbf{p})-\varepsilon_{v}(\mathbf{p})$, where $c$ and $v$ denote the conduction and valence band, 
respectively. The system Hamiltonian has several terms which differ from gas ionization

$$
H=H_{\text {elec }}+H_{\text {latice }}+H_{\text {elec-lattice }}+H_{\text {rad }}
$$

where $H_{\text {dec }}, H_{\text {lattice }}, H_{\text {dec-iattice }}, H_{\text {mad }}$ are the Hamiltonians for the electron, the lattice, the electron-lattice interaction, and the radiation field, respectively. Instead of the free electron wavefunction with an applied field found in gas ionization, Eq. (3.12), we now have a solid state Bloch wavefunction in a field

$$
\begin{aligned}
\psi_{p}^{c, v}(\mathbf{r}, t) & =u_{p(t)}^{c, v}(\mathbf{r}) \exp \left\{\frac{i}{\hbar}\left[\mathbf{p}(t) \mathbf{r}-\int_{0}^{t} \varepsilon_{c, v}(\mathbf{p}(\tau)) d \tau\right]\right\} \\
\mathbf{p}(t) & =\mathbf{p}+(e \mathbf{F} / \omega) \sin \omega t
\end{aligned}
$$

where $u_{p(t)}^{c, v}(\mathbf{r})$ are periodic functions with the lattice translational symmetry. The calculations are similar to the gas ionization process above except that a dispersion relation must be used, e.g., for semiconductors $\varepsilon(p)=\Delta\left(1+p^{2} / m \Delta\right)^{1 / 2}$ where $\Delta$ is the width separating the valence and conduction bands, and $1 / m=1 / m_{e}+1 / m_{h}$ is the reciprocal of the reduced mass of the electron and the hole. The final ionization probability is similar in form to the gas ionization formula, Eq. (3.13)

$$
\begin{array}{r}
w=\frac{2 \omega}{9 \pi}\left(\frac{\sqrt{1+\gamma^{2}}}{\gamma} \frac{m \omega}{\hbar}\right)^{3 / 2} Q\left(\gamma, \frac{\bar{\Delta}}{\hbar \omega}\right) \exp \left\{-\pi\left(\frac{\tilde{\Delta}}{\hbar \omega}+1\right)\right. \\
\left.\times\left[K\left(\frac{\gamma}{\sqrt{1+\gamma^{2}}}\right)-E\left(\frac{\gamma}{\sqrt{1+\gamma^{2}}}\right)\right] / E\left(\frac{1}{\sqrt{1+\gamma^{2}}}\right)\right\}
\end{array}
$$

where $\bar{\Delta}$ is the effective ionization potential

$$
\tilde{\Delta}=\frac{2}{\pi} \Delta \frac{\sqrt{1+\gamma^{2}}}{\gamma} E\left(\frac{1}{\sqrt{1+\gamma^{2}}}\right)
$$

and $Q$ is analogous to the function $S$ in the gas ionization formula, Eq. (3.14). 


\subsection{ULTRA-SHORT PULSE INTERACTION WITH A SOLID}

Ionization by strong fields occurs on the femtosecond timescale, so the majority of the pulse interaction with the solid is treated as the interaction of electromagnetic waves and a plasma $[95,96]$. The interaction is completely described by Maxwell's equations

$$
\begin{aligned}
& \nabla \times \mathbf{H}=\frac{4 \pi}{c} \mathrm{j}+\frac{i \omega}{c} \mathrm{D} \\
& \nabla \cdot \mathrm{D}=4 \pi \rho \\
& \nabla \times \mathrm{E}=-\frac{i \omega}{c} \mathrm{H} \\
& \nabla \cdot \mathrm{H}=0
\end{aligned}
$$

with $\mathrm{D}=\varepsilon(\omega) \mathrm{E}$ and $\mathbf{j}=\sigma(\omega) \mathrm{E}$, where $\mathrm{H}$ and $\mathrm{D}$ are the electric and magnetic displacement, $\mathrm{j}$ and $\rho$ are the free current and charge densities, $E$ is the electric field, and $\varepsilon(\omega)$ and $\sigma(\omega)$ are the dielectric constant and the conductivity. From Maxwell's equations, the wave equations which describe the electromagnetic field propagation in the plasma are easily derived $[95,96]$

$$
\begin{aligned}
& \nabla^{2} \mathbf{E}-\nabla(\nabla \cdot \mathbf{E})+\frac{\omega^{2}}{c^{2}} \varepsilon \mathbf{E}=0 \\
& \text { and } \\
& \nabla^{2} \mathbf{H}+\frac{\omega^{2}}{c^{2}} \varepsilon^{\prime} \mathbf{H}+\frac{1}{\varepsilon^{\prime}} \nabla \times(\nabla \times \mathbf{H})=0
\end{aligned}
$$

The dispersion relation for electromagnetic waves in a plasma comes directly from these wave equations [96]. In a homogeneous plasma $\nabla \varepsilon^{\prime}=0$ and $\nabla \bullet E=0$, so a field that behaves as $\exp (i \mathbf{k} \bullet \mathbf{x})$ will have a dispersion relation (from Eq. (3.22)) of

$$
\frac{\omega^{2}}{c^{2}} \varepsilon^{\prime}=k^{2} \text { or } \omega^{2}=\omega_{p}^{2}+k^{2} c^{2}
$$

Implicit in these equations is neglect of spatial dispersion $(\varepsilon(\omega, k)=\varepsilon(\omega))$ or treating the plasma as a cold plasma where thermal motion may be neglected. Spatial dispersion arises from the charge carrier inertia and the friction, so that the medium polarization is not only determined by the field at that instant. If the 
characteristic quantity $k D \ll 1$, where $k=2 \pi / \lambda$ is the laser wavenumber, and $D=\sqrt{k_{B} T / 8 \pi e^{2} n_{e}}$ is the Debye length, then spatial dispersion can be neglected. In our case $k D \sim 10^{-3}$, so there is no difficulty with this approximation. Also implicit in these equations is the treatment of the plasma as isotropic, so the constitutive equations between $\mathrm{D}$ and $\mathrm{E}$ or $\mathbf{j}$ and $\mathrm{E}$ are not tensor relations. Moreover, the plasma medium is assumed to be non-magnetic (magnetic permeability $=1$ ), which is another effect of ignoring spatial dispersion. The field equations are assumed to be linear. That is, the higher order, field-dependent parts of $\varepsilon(\omega)$ and $\sigma(\omega)$ are ignored. We will discuss this approximation later in Chapter 4 when the model is compared to experimental results.

Finally, is a treatment using macroscopic fields valid in this problem? The mean macroscopic fields have been taken to be the fields acting on the plasma electrons. However, for an isotropic medium, there is an effective field arising from the macroscopic field and the surrounding charges $\mathrm{E}_{\text {off }}=\mathrm{E}+4 \pi a \mathrm{P}$, where $P$ is the polarization and $a$ is a coefficient which depends on the medium details. The extra polarization term is often called the Lorentz term. In a solid, for example, if the molecules are treated as randomly-oriented point dipoles it can be shown that $a=1 / 3$ [95]. In a plasma, however, the Lorentz term can be shown, in a lengthy proof, to be zero $[112,113]$, so the macroscopic field and the effective field are the same.

Two questions arise in our treatment of the field-matter interaction: are the field propagation details important to the reflectivity calculations, since we regard the plasma as having nearly negligible expansion anyway?, and (2) if the propagation is important and given the wave equations above, what does the electromagnetic field look like inside the plasma created by the pulse's leading edge? 
If the density profile is a perfect step function, Fresnel formulas [2] give characteristic reflectivity curves as a function of incident angle that are dependent only on the local plasma index of refraction. The local plasma index depends both on the plasma density and temperature, as described below. Although the initial density profile of an ultra-short pulse plasma is nearly a step function, it has been found that even a slight expansion on the order of $0.01 \lambda$ will significantly alter reflectivity $[3,98,114]$. In this case, Fresnel reflectivity is replaced by a more exact solution from solving the Helmholtz wave equations numerically.

Since we see that propagation is important, even in short-scale-length plasmas, we now answer the second question and look at basic field propagation characteristics. Propagation solutions are covered in detail by Kruer [96], so only some results will be quoted here. An electromagnetic wave will propagate up the density gradient until, as seen in the dispersion relation Eq. (3.23), it reaches critical density where electrons shield out the field. Beyond this density, the light wave is exponentially attenuated. On propagation towards critical density, the local group velocity $v_{g}=c \sqrt{\varepsilon(\omega, z)}$ decreases at higher densities and the field swells. Field swelling is physically the result of conservation of energy flux, i.e.,

$$
\frac{v_{g}|E(z)|^{2}}{8 \pi}=\frac{c E_{\text {free }}^{2}}{8 \pi}
$$

where $E(z)$ and $E_{\text {free }}$ are the electric field in the plasma at position $z$ and in free space, respectively. An analytic example illustrates the effect [96]. If the plasma density varies linearly along the $z$ direction as $n=n_{c r} z / L$, the solution to the wave equation for the electric field of a normally incident wave goes as an Airy function, $E(z) \sim A_{i}(z)$. When the wave is obliquely incident, essentially the same propagation pattern is followed except the wave reflects at a lower density due to refractive turning in the plasma. In the case of oblique incidence, S- and P- 
polarized waves must be considered separately. A P-polarized wave can excite a plasma wave, or plasmon, near the critical surface because its electric field will have a component oscillating along the plasma density gradient. At the critical surface, the field excites the plasma resonance $\left(\omega=\omega_{p}\right)$ and field energy is converted to electrostatic wave energy, so there is strong P-polarized light absorption. This is one reason these experiments were mainly done with $\mathrm{S}$ polarization. The small blowoff plasma is supercritical, but below solid density, so P-polarized light will be strongly absorbed before it has a chance to penetrate the plasma deeper into the solid density region. S-polarized light does not experience resonance absorption, so it is a better probe of solid density plasmas. In addition, the complication of calculating the amount of resonance absorption with P-polarized light makes S-polarized light a more desirable probe.

Complex plasma permittivity used in the propagation equations above is determined from plasma temperature and density with the following model. Reflective properties of a plasma depend directly and completely on the complex permittivity (see Eq. (3.22))

$$
\varepsilon^{\prime}(\omega)=\varepsilon(\omega)-i(4 \pi / \omega) \sigma(\omega)
$$

In the general case, expressions for $\varepsilon(\omega)$ and $\sigma(\omega)$ are derived using kinetic theory from the Boltzmann equation. However, if we assume the collision frequency is independent of electron velocity, a constant effective collision frequency, $v_{f f f}$, can be used [95]. This elementary theory is presented in Ginzburg [95] and gives the expressions

$$
\begin{aligned}
& \varepsilon^{\prime}(\omega)=1-\frac{4 \pi e^{2} n_{e}}{m \omega\left(\omega-i v_{e f f}\right)} \\
& \varepsilon(\omega)=1-\frac{4 \pi e^{2} n_{e}}{m\left(\omega^{2}+v_{e f f}{ }^{2}\right)} \text { and } \sigma(\omega)=\frac{e^{2} n_{e} v_{e f f}}{m\left(\omega^{2}+v_{e f f}{ }^{2}\right)}
\end{aligned}
$$


From this complex permittivity, plasma reflectivity for a given density and temperature can be calculated with Fresnel or Helmholtz equations. The density effect is explicit in the permittivity, but it is also implicit because the collision frequency is a function of density. For example, the standard Spitzer [115] electron-ion collision frequency is

$$
v=\frac{4 \sqrt{2 \pi}}{3} \frac{Z n_{e} e^{4} \ln \Lambda}{m^{1 / 2} T_{e}^{3 / 2}}
$$

where $Z$ is the average ion charge and $\ln \Lambda$ is the Coulomb logarithm. The Coulomb logarithm describes the maximum to minimum impact parameter ratio in plasma electron-ion collisions [96]. Electron temperature also affects the permittivity through the collision frequency as shown in Eq. (3.27) above. 


\section{CHAPTER 4:}

\section{SPACE-AND TIME-INTEGRATED REFLECTIVITY MEASUREMENTS}

\subsection{INTRODUCTION}

The time-integrated, specular reflectivity of plasmas, created by the laser source described in Chapter 2, was measured as a function of laser intensity. Targets included an array of materials $\left(\mathrm{Al}, \mathrm{Au}, \mathrm{Si}, \mathrm{SiO}_{2}\right)$ with widely differing initial electronic properties. Reflectivity measurements and associated modeling were done to improve the understanding of ultra-short pulse plasmas in several ways: (1) to provide a consistent database for the reflectivity of plasmas formed in materials with widely differing electronic properties under identical experimental conditions, (2) to determine the relative importance of avalanche and tunneling ionization to initial plasma formation under strong-field, ultrashort pulse conditions, (3) to study the role of nonlinear plasma heating at high intensities, and (4) to explore the nonequilibrium nature of an initial plasma state created by tunneling ionization. All of these points were also valuable to understanding time-resolved measurements introduced in Chapter 5.

Reflectivity measurements described in other works $[3,4,13,114,116,117]$ were performed under disparate experimental conditions. As such, they do not present a coherent picture of intensity-dependent reflectivity as a function of a material's initial electron density and electronic properties. The measurements here give a consistent picture of plasma features including absorption, ionization and absolute, high-field reflectivity. In addition, to our knowledge, there has been no integrated reflectivity measurement for a wide-band-gap insulator under intense, ultra-short pulse illumination. This is an important gap in 
understanding these plasmas, because insulators emphasize an interesting feature of strong-field environments, tunneling ionization.

This chapter mainly focuses on the reflectivity response of two metals (Al and $\mathrm{Au})$ and an insulator $\left(\mathrm{SiO}_{2}\right)$. Data on metals is primarily determined by absorption, since the plasma frequency of both the initial solid and ensuing plasma is above optical frequencies. Light is either absorbed by inverse Bremsstrahlung and other mechanisms, or reflected. Ionization plays a smaller role in the reflectivity. We therefore use absorption to explain the metals' reflectivity and infer plasma density, collisionality, temperature, average ionization state, expansion velocity, and scale length. Strong-field additions and modifications to inverse Bremsstrahlung absorption physics are accounted for. Insulator reflectivity, on the other hand, is extremely sensitive to electron density and, to a smaller degree, electron temperature. Because the insulator is sensitive to ionization, $\mathrm{SiO}_{2}$ data is used to test a prediction that high-intensity, ultra-short pulse plasmas form mainly by tunnel ionization and, furthermore, can exist in a transient state which is not in LTE. The test is to compare reflectivity results to a tunnel-ionized, non-LTE plasma model.

Reflectivity from the semiconductor target $(\mathrm{Si})$ is shown for continuity and comparison to published data. This data agrees very well with that of Murnane [6], who studied Si reflectivity over an intensity range $\sim 10^{12}-2 \times 10^{15} \mathrm{~W} / \mathrm{cm}^{2}$ with S-polarized, $160 \mathrm{fs}, 616 \mathrm{~nm}$ pulses at a $5^{\circ}$ incident angle. A detailed analysis of $\mathrm{Si}$ reflectivity is given by Murnane [6]. The Si response lies, as one might expect, somewhere between metals and insulators. The cold electron density of $\mathrm{Si}$ $\left(-10^{18} \mathrm{~cm}^{-3}\right)$ is below critical density, so ionization is important to the reflectivity. On the other hand, the electron density begins high enough for significant inverse Bremsstrahlung absorption to occur throughout the laser-matter interaction. 
Two possible mechanisms exist for initial formation of high-density plasma by a high-intensity, subpicosecond pulse: (1) avalanche ionization and (2) multiphoton or tunneling ionization. Bloembergen [14] predicted tunneling ionization would dominate avalanche ionization and become the breakdown mechanism for subpicosecond pulses. Time-integrated reflectivity measurements in this work confirm this prediction.

We compare a model which incorporates both avalanche and tunneling ionization to $\mathrm{SiO}_{2}$ reflectivity measurements and identify tunneling as the active mode of ionization in ultra-short pulse plasmas. Time-integrated reflectivity of a sharp-interface, high-density plasma depends only on the electron temperature, the electron density and the average ionization state, $Z$. This relationship is immediately seen from the plasma conductivity Drude formalism presented below which directly determines the reflectivity. A model determining electron density and temperature, in a self-consistent manner, along with a method for determining $Z$ should thus be sufficient for predictions based on reflectivity. The avalanche breakdown picture predicts an exponential growth in density with a timescale longer than observed by reflectivity measurements. The tunneling picture, however, has ionization rates consistent with observed reflectivities.

Furthermore, our model goes beyond confirming Bloembergen's prediction by examining important consequences of a tunnel ionized plasma. Using rate equations to determine plasma density, rather than relying on a predicted LTE density which assumes a Saha equilibrium state, we investigate the nonequilibrium nature of plasmas formed with ultra-short pulses. We find that photo-ionized plasmas produced by the interaction of an intense, ultra-short laser pulse with a solid can form out of local thermodynamic equilibrium (LTE) during the pulse's rising edge. Photo-ionization causes the plasma to form out of 
LTE because the photo-ionization rate exceeds the rate at which electron-ion collisions can equilibrate ion states to a distribution predicted by the Saha equation. We propose a self-consistent model with closed-form solutions for electron density and temperature, valid during interaction with a high-intensity pulse. The model gives simple estimates of electron densities and temperatures as well as physically intuitive scaling laws. Electron temperature and density results are used in a Drude model to determine evolving plasma conductivity. Calculated conductivities predict measurable time-integrated and time-resolved plasma reflectivity, absorption, and transmission. Calculated reflectivities as a function of intensity are compared to time-integrated data. The model is shown to agree very well with measurements of time-integrated plasma reflectivity using fused quartz $\left(\mathrm{SiO}_{2}\right)$ targets.

\subsection{SELF-REFLECTIVITY EXPERIMENTAL SETUP}

The time-integrated reflectivity experiment monitored the self-reflectivity of high-intensity, ultra-short pulses as a function of intensity. A schematic experimental set-up is shown in Figure 4.1. The input beam was the $3.5 \mathrm{~mJ}$, $614 \mathrm{~nm}, \sim 130 \mathrm{fs}$ source described in Chapter 2 incident on a solid target at an angle of $15 \pm 2^{\circ}$ to the target normal. Targets consisted of $1.5^{\prime \prime} \times 1.5^{\prime \prime} \times 0.0625^{\prime \prime}$ squares of uncoated fused quartz or fused quartz coated with a $1 \mu \mathrm{m}$ layer of $\mathrm{Al}, \mathrm{Au}$, or $\mathrm{Si}$. A $1 \mu \mathrm{m}$ coating was much larger than the ionized target skin depth, $\delta=\sqrt{2 \varepsilon_{0} c^{2} / \sigma \omega} \sim 100 \AA$, so the coating was considered a semi-infinite material. Target surfaces were prepared by cleaning with acetone and methanol immediately before use. All targets were placed in a 1 $1 \mu$ Torr vacuum on a target wheel and rotated into the beam by computer control, so measurements were taken under identical conditions without breaking vacuum. Measured variation 
in target position as the wheel was rotated was smaller than the beam confocal parameter. A $10 \mathrm{~cm}$ achromatic lens focused the input beam while a $12 \mathrm{~cm}$ lens collected specularly reflected light. A restrictive experimental geometry allowed only this nonconfocal arrangement. The optimum focusing lens position was determined by maximizing both reflectivity and the signal from an $x$-ray diode placed near the target. Laser polarization was changed between $S$ and $P$ by a half wave plate and was estimated to be $\sim 98 \%$ linear.

An electronic shutter chopped the beam to a $5 \mathrm{~Hz}$ repetition rate, so shots were spaced more than $100 \mu \mathrm{m}$ apart by a target raster scanning system. The raster scanning system was a set of Klinger stepper motor translation and rotation stages with $0.1 \mu \mathrm{m}$ resolution. Incident and reflected energies were recorded shot-to-shot by Molectron J3-09 $\mathrm{Si}$ energy probes connected to a Molectron JD-2000 ratiometer. Spectra were recorded by a McPherson spectrometer (model 270, 0.35m, 1200 lines/mm), EG\&G optical multichannel analyzer (model 1461) and diode array detector $(0.4 \AA /$ pixel) controlled by the Macintosh MacOMA program. By inserting a mirror in the beam, pulsewidth was monitored with a home-built, second-order, single-shot autocorrelator. The autocorrelation signal was detected by an EG\&G Reticon 1024 element diode array (model RL1024) and recorded by a HP 54502 digital oscilloscope connected to the computer. The focal spot was determined by sampling the reflected beam with a pellicle with the target at $0^{\circ}$ using a Pullnix camera (model TM 745E) and magnifying-imaging system. The focal spot was recorded on a Spiricon LBA-100 beam profiler. The absolute focal spot diameter was determined by comparison with an image of a Newport RES-1 resolution target with appropriate dark and gain levels set on the beam profiler. All signal detection, data recording, raster scanning, and shutter operation was synchronized by a Labview 2 virtual instrument program interfaced with a Macintosh IIfx computer by GPIB protocol. 


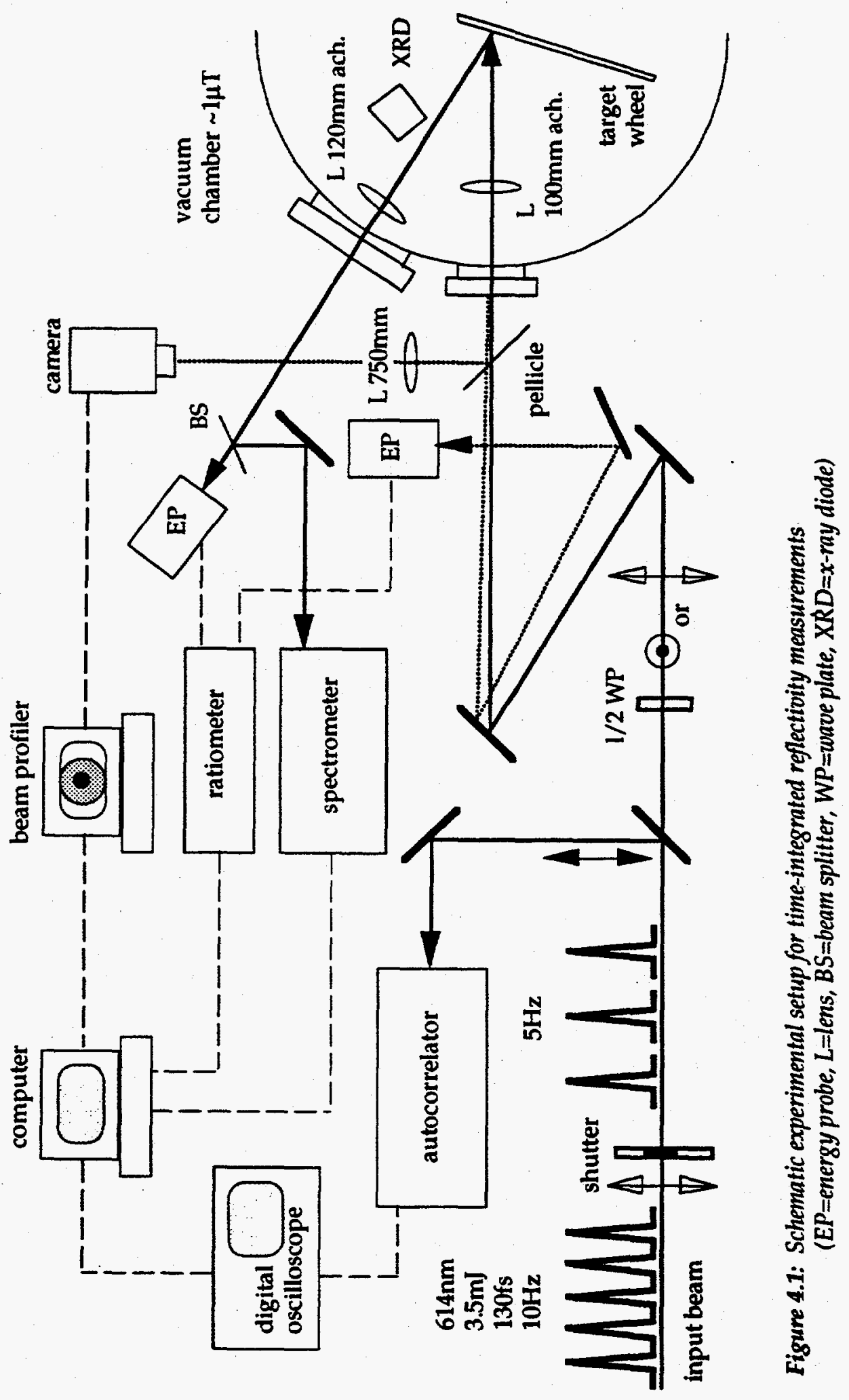




\subsection{EXPERIMENTAL RESULTS}

\subsubsection{Energy Reflectivity Measurements}

Self-reflectivity versus intensity results for metals ( $\mathrm{Al}$ and $\mathrm{Au}$ ), a semiconductor $(\mathrm{Si})$ and an insulator $\left(\mathrm{SiO}_{2}\right)$ are shown in Figures $4.2(\mathrm{a})-(\mathrm{h})$. Single-shot measurements were taken with S- and P-polarized light under identical experimental conditions facilitated by using a target wheel.

The striking difference in the intensity-dependent reflectivity behavior identifies the sensitivity of the laser-matter interaction to initial cold electron density. At the lowest intensities, $\sim 10^{12}-10^{13} \mathrm{~W} / \mathrm{cm}^{2}$, the amount of field penetration into the material differentiates the response of metals, semiconductors, and insulators. Plasma generation is limited, so the response is primarily determined by solid state details. In the presence of absorption mechanisms, absorption is large in materials which allow deep field penetration. The skin depth of metal is small, $\sim 10 \mathrm{~nm}$ for visible light, so field penetration is minimal. On the other hand, fields readily propagate through the entire bulk of a transparent insulator. Reitze [118] showed evidence of strong nonlinear absorption in Si at intensities up to $5 \times 10^{11} \mathrm{~W} / \mathrm{cm}^{2}$. Primary interband absorption mechanisms are multi-photon or carrier-assisted absorption, and band gap renormalization [118]. The supercritical density of the metals simply does not allow absorption because most of the light is reflected away before it has a chance to be absorbed. Insulators absorb in a large, underdense bulk volume and also transmit readily. Semiconductors lie in the intermediate regime.

Note that the reflectivity of all the materials approaches a common range of values, $\sim 70-80 \%$ (S polarization) and $\sim 55-60 \%$ ( $\mathrm{P}$ polarization, $15^{\circ}$ ), at the highest intensities. This indicates that the laser field interacts with a supercritical plasma state common to all the materials. It is not sensitive to cold ions from 
vastly different materials. We believe this is the first observation of a materialindependent plasma reflectivity law occurring when materials reach a common state at an intensity $>10^{15} \mathrm{~W} / \mathrm{cm}^{2}$. The mechanism to reach this common state may be a high-intensity version of the self-regulating plasma absorption regime found in long pulse plasmas [119]. This reflectivity behavior has been independently confirmed at this laboratory [120] over a large intensity range $\left(10^{13}-10^{18} \mathrm{~W} / \mathrm{cm}^{2}\right)$ with $\sim 800 \mathrm{~nm},-100$ fs laser pulses using glass, $\mathrm{Ta}, \mathrm{Cu}, \mathrm{Au}$, and Al targets. The material-independent plasma state identified in this work at intensities from $\sim 10^{15}-10^{16} \mathrm{~W} / \mathrm{cm}^{2}$ was found to extend to $\sim 10^{18} \mathrm{~W} / \mathrm{cm}^{2}$ because all targets showed common qualitative (monotonically decreasing) and quantitative $( \pm 5 \%)$ absorption [120]. Furthermore, note that the metals and the semiconductor reach a reflectivity minimum at $\sim 10^{14} \mathrm{~W} / \mathrm{cm}^{2}$, after which reflectivity begins to rise slightly. This indicates resistivity saturation, as discussed by Milchberg [3]. At this minimum, plasma has reached a state where electron-ion collisions occur with a mean free path approximately equal to interatomic spacing. This is the shortest possible distance between collisions. Beyond this intensity, the temperature rises, which lowers collision cross-sections and resistivity. At the highest intensities, high-field (nonlinear) absorption effects also reduce absorption. The mechanisms behind these nonlinear effects and quantitative estimates are discussed in Section 4.3.3. The slight upturn in the reflectivity data at the highest intensity may indicate this nonlinear absorption, estimated to become significant at $>1015 \mathrm{~W} / \mathrm{cm}^{2}$ [98]. 


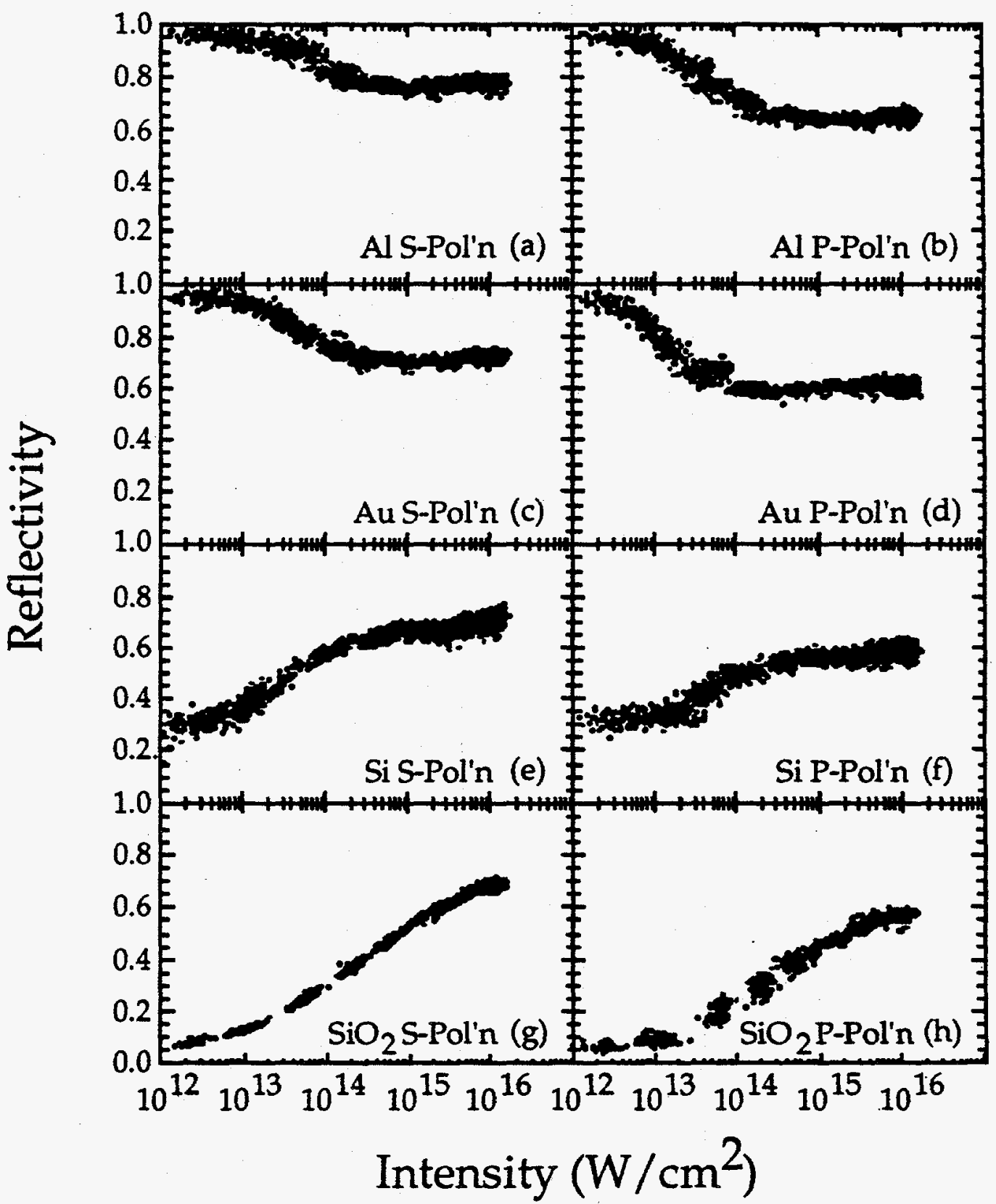

Figure 4.2: Time-integrated reflectivity oersus intensity for metals ( $A l$ and $A u$ ), a semiconductor (Si), and an insulator $\left(\mathrm{SiO}_{2}\right)$ with S- and P-polarized light. 


\subsubsection{Cold Target and High Prepulse Measurements}

Cold target reflectivity at low intensities was measured on each target directly in air for comparison to reflectivity calibration measurements. Direct measurements agreed with calibration measurements and tabulated values to within $1 \%$ over the full range of angles shown in Figure 4.3.

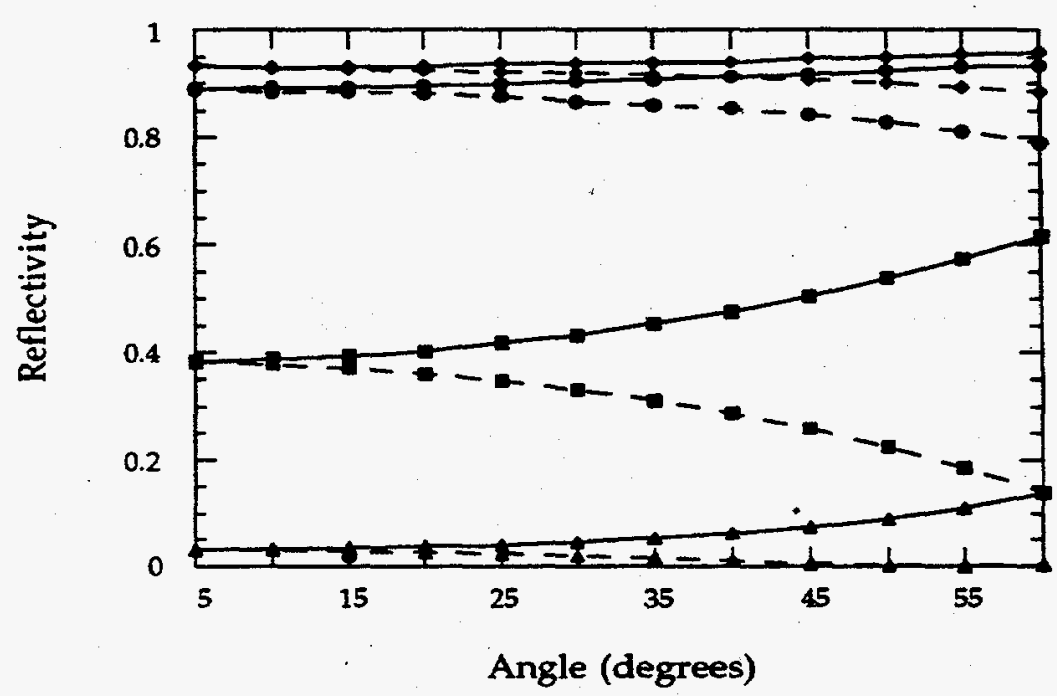

Figure 4.3: The measured cold target reflectivity for the Al (circle), Au (diamond), Si (square) and $\mathrm{SiO}_{2}$ (triangle) targets for $\mathrm{S}$ (solid) and $\mathrm{P}$ (dash) polarization.

In addition, reflectivity was measured as a function of intensity with an artificially high ASE energy introduced. ASE energy was raised to $\sim 3 \%$ by removing the saturable absorber in the bowtie amplifier. This prepulse energy created a small preplasma and provided an additional test for excessive prepulse by showing its effect on reflectivity. High prepulse results are shown in Figure 4.4. Absorption from a small preplasma is clearly seen at higher intensities. Results are much more similar to long pulse (>ps) experiments [6] where absorption is near $90 \%$ as described in Chapter 3. 


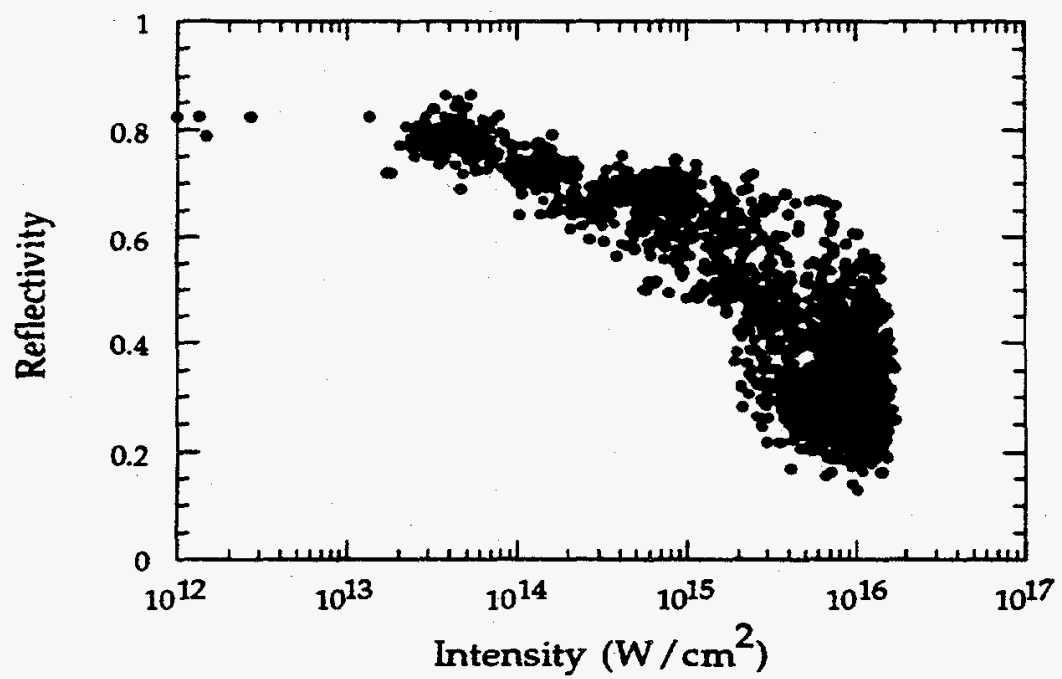

Figure 4.4: The reflectioity of an Au target with 3\% ASE prepulse energy introduced.

\subsubsection{Analysis of Results: The Metals $\mathrm{Al}$ and $\mathrm{Au}$}

What significant physical processes determine reflectivity for the metals ( $\mathrm{Al}$ and $\mathrm{Au}$ ) in Figures 4.2 (a)-(d)? As discussed in Chapter 3, femtosecond plasma reflectivity depends primarily upon electron temperature (inverse Bremsstrahlung absorption) and density (critical density formation and inverse Bremsstrahlung absorption). Parametric plasma instabilities mentioned in Section 3.2.1 do not play a role. High intensities, however, do introduce nonlinear (intensity-dependent) factors to inverse Bremsstrahlung absorption, as discussed in Section 4.3.3.2. Plasma scale length (although small) is important, because a P-polarized field resonantly couples to critical plasma density exciting electrostatic plasma waves $[95,121-123]$. Even a small scale length causes significant absorption when plasma waves collisionally damp and leave the interaction region. The physics and magnitude of collisional "resonance absorption" is discussed in Section 4.3.3.1. Moreover, P-polarized radiation also induces a collisionless vacuum heating $[124,125]$ predicted to be more important 
than resonance absorption at high irradiance in short scale-length plasmas. Vacuum heating occurs when electrons are dragged out of the plasma and into vacuum by a strong electromagnetic field. Electrons are then sent back into the plasma, as the field changes sign, where they deposit their kinetic energy at the electron quiver velocity. Details are given in Section 4.3.3.2.

We explain reflectivity of S-polarized radiation in $\mathrm{Al}$ and $\mathrm{Au}$ using only the linear and nonlinear inverse Bremsstrahlung absorption. P-polarized radiation adds collisional resonance absorption and collisionless vacuum heating to this picture. Absorption mechanisms, such as sheath inverse Bremsstrahlung $[126,127]$ and "skin" effects [128-130] are ignored because they become significant at higher intensities $\left(>10^{16} \mathrm{~W} / \mathrm{cm}^{2}\right)$, or when the scale length is zero $[125,131$, 132]. Analytic calculations are used to estimate absorption for comparison to the data at the highest intensities $\left(-10^{15}-10^{16} \mathrm{~W} / \mathrm{cm}^{2}\right)$ in Figures 4.2 (a)-(b). A computational model (LASNEX) is also used in Section 4.3.3.4 for comparison over the full data intensity range.

\subsubsection{Analytic Calculations}

The metals, $\mathrm{Al}$ and $\mathrm{Au}$, have cold electron densities of $18.1 \times 10^{22} \mathrm{~cm}^{-3}$ and $5.90 \times 10^{22} \mathrm{~cm}^{-3}$, respectively, which are above the critical density $2.92 \times 10^{21} \mathrm{~cm}^{-3}$ $(\lambda=614 \mathrm{~nm})$. The field inside the plasma is, therefore, evanescent and reduced by a factor $\sim \omega / \omega_{p}$, so the maximum field intensity is $<10^{15} \mathrm{~W} / \mathrm{cm}^{2}$ beyond the surface electrons. Multiple ionization is significantly reduced in the bulk metal by the shielding. However, a cold, dense sea of electrons will show significant inverse Bremsstrahlung absorption, because the electron-ion collision frequency behaves as $v_{e-i} \sim n_{e} Z / T_{e}^{3 / 2}$. 
Plasma scale length and collisionality at the highest intensities were estimated from S-polarized reflectivity values ( 70\% for $\mathrm{Au}, \sim 75 \%$ for $\mathrm{Al})$ by comparison with the Helmholtz wave solving model of Fedosejevs [114]. Fedosejeves' results predict reflectivity as a function of scale length, collisionality, and incidence angle. We obtain values of $L / \lambda \approx 0.01, v_{e-i} / \omega \approx 0.04$ for $\mathrm{Al}$ and $L / \lambda \approx 0.01, v_{e-i} / \omega \approx 0.05$ for $\mathrm{Au}$. These values agree well with short pulse plasma scaling arguments presented in Chapter 3.

From the collision frequency, we obtain an estimate for electron temperature. Collisionality is related to temperature by [115]

$$
v_{e-i}=\frac{4 \sqrt{2 \pi}}{3} \frac{Z n_{e} e^{4} \ln \Lambda}{m^{1 / 2} T_{e}^{3 / 2}}
$$

where $\ln \Lambda=\ln \left(\lambda_{D} / r_{\min }\right)$ is the Coulomb logarithm, $\lambda_{D}=\left(T_{c} / 4 \pi m_{e} e^{2}\right)^{1 / 2}$ is the plasma Debye (screening) length, and $r_{\min }$ is the larger of the classical distance of closest approach, $Z e^{2} / m v^{2}$, and the deBroglie wavelength, $\hbar / 2 m v$.

To establish the average ion charge state, $Z$, for this calculation, a plasma model must be employed. Laser-produced plasmas are generally modeled with one of several plasma kinetics models: (1) a coronal model, valid for low density plasmas where radiative processes dominate over collisional physics, (2) a collisional-radiative model, valid at densities $\sim 10^{21} \mathrm{~cm}^{-3}$, where both collisional and radiative processes are important, (3) a local thermodynamic equilibrium (LTE) model, valid for densities $-10^{23} \mathrm{~cm}^{-3}$, where collisions control the atomic processes, or (4) a non-LTE (NLTE) model that uses rate equations to explicitly calculate $Z$, density, and temperature. An LTE model is sufficient when inverse Bremsstrahlung absorption at high densities is the main process modifying the reflectivity. The LTE [97] model treats the free electron distribution as Maxwellian, the occupation of ionic levels as a Boltzmann distribution, and the ion state distribution as being in Saha equilibrium. When we consider the 
ionization of an insulator below, we use an NLTE model because ionization, not absorption, is a critical reflectivity modifier.

For the metals, we take a simpler approach to calculate $Z$, the ThomasFermi model [133]. This does not treat the plasma with kinetic LTE or NTLE theory. The Thomas-Fermi model does not distinguish between free and bound electrons. The nuclei obey Boltzmann statistics and contribute separately to pressure and internal energy like a monatomic gas. Total particle interaction energy is associated with electrons only. Matter "cells" containing $Z$ electrons and a nucleus exert pressure on each other equal to an electron gas pressure. This repulsive force model gives reasonable results for high densities, compressed solids or at high temperatures where coupling forces can be neglected and repulsive forces dominate.

From the Thomas-Fermi results for $Z$, we calculate the temperaturedependent collision frequency at critical density normalized to the laser frequency. Electron temperature is then inferred by matching the calculated, temperature-dependent collision frequency to the result obtained from the Helmholtz wave equation model. Results are shown in Figure 4.5. From the collisionality found for $\mathrm{Al}\left(v_{e-i} / \omega \approx 0.04\right)$ and $\mathrm{Au}\left(v_{e-i} / \omega \approx 0.05\right)$, inferred temperatures at the highest intensities are $\sim 100-120 \mathrm{eV}$ for the $\mathrm{Al}$ target and $\sim 150-$ $170 \mathrm{eV}$ for the Au target.

Furthermore, using values for collision frequency and scale length, we calculated fractional linear inverse Bremsstrahlung absorption, $f_{I B}$, derived by the method of Kruer [96]

$$
f_{I B}=1-\exp \left(-\frac{8 v_{e-i}\left(n_{c}\right) L}{3 c} \cos ^{3} \theta\right)
$$

where $v_{e-i}\left(n_{c}\right)$ is the critical density collision frequency, $\theta$ is the angle of incidence, and $L$ is the plasma scalelength. 

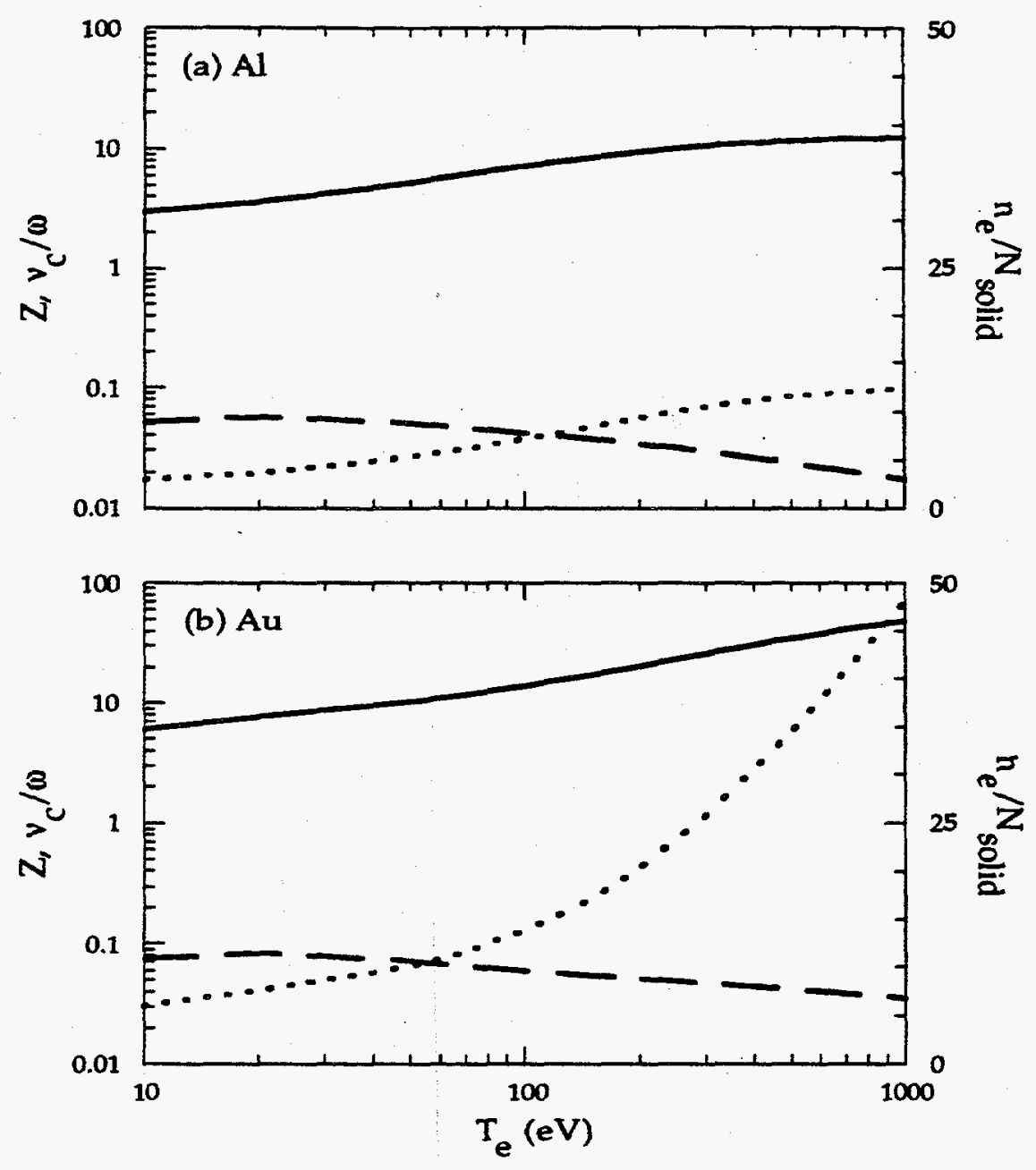

Figure 4.5: Average ion charge state (solid), normalized collision frequency at critical density (dash), and normalized electron density (dot) oersus temperature for (a) Al and (b) Au.

Calculated percentage linear inverse Bremsstrahlung absorption is shown in Figure 4.6. Figure 4.6 also shows the percentage absorption for P-polarized light due to resonance absorption which is discussed below. For S-polarized light, calculated absorption at inferred temperature ranges is $\sim 21-23 \%$ for $\mathrm{Al}$ and 28-30\% for $\mathrm{Au}$, in fair agreement with observed values of $25 \%$ and $30 \%$, respectively. For P-polarized light, calculated absorption (inverse Bremsstrahlung + resonance) at inferred temperature ranges is $\sim 27-29 \%$ for $\mathrm{Al}$ 
and $-32-35 \%$ for $\mathrm{Au}$, also in fair agreement with observed values of $35 \%$ and $40 \%$, respectively. Note, however, observed values include both absorption and scattered light that will be accounted for below. Moreover, these values do not reflect nonlinear (intensity-dependent) collisional absorption or collisionless vacuum heating effects, which are treated in Section 4.3.3.2.
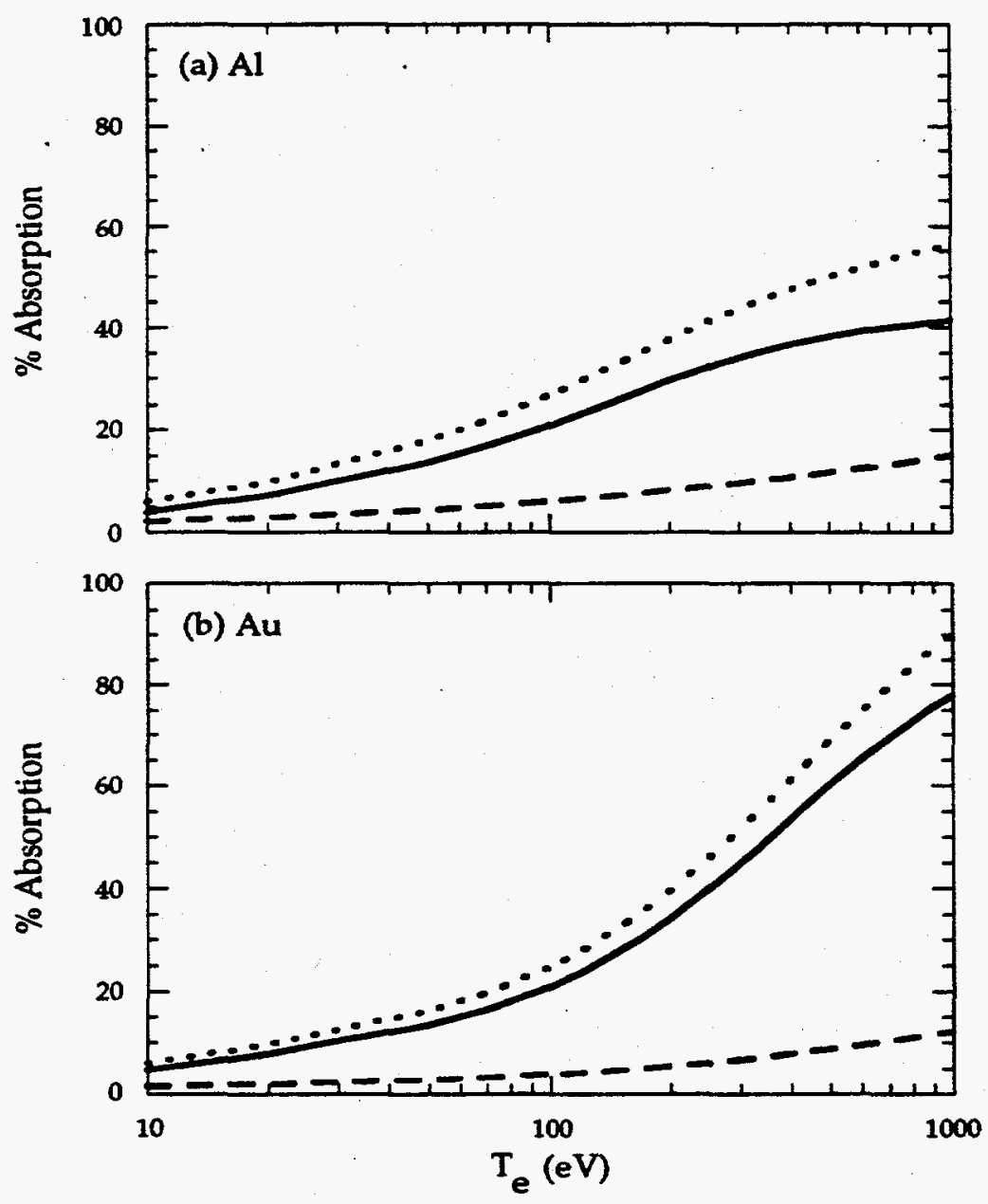

Figure 4.6: The percentage linear inverse Bremsstrahlung absorption (solid) for Spolarized light and the percentage resonance absorption (dash) and total absorption = inverse Bremsstrahlung + resonance absorption (dot) for P-polarized light versus temperature for the (a) $A l$ target and (b) Au target. Resonance absorption is discussed below. 
The temperature estimate obtained from Figure 4.5 was compared to an independent temperature measurement using reflected laser pulse spectral shifting [134, 135]. In the process of estimating temperature from spectral shifting, plasma expansion velocity, scale length and percentage resonance absorption were calculated, as detailed below. A typical spectral shift of $3.3 \AA$ is seen at $\sim 10^{15} \mathrm{~W} / \mathrm{cm}^{2}$ using an $\mathrm{Al}$ target as shown in Figure 4.7.

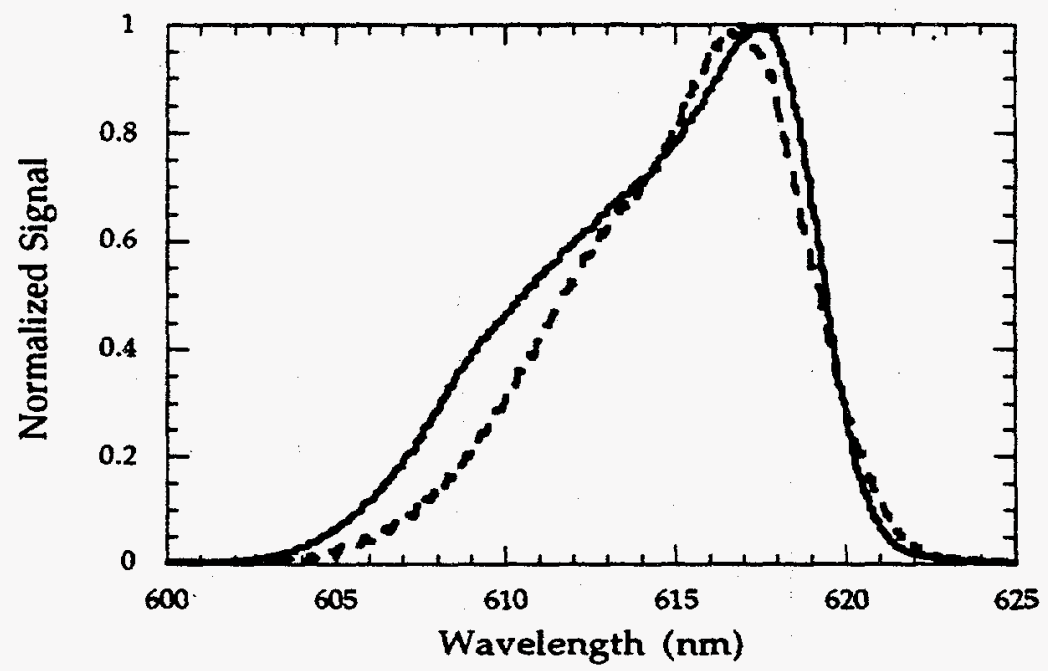

Figure 4.7: The reflected spectrum peak (dash) was Doppler-shifted to shorter wavelengths than the incident spectrum peak (solid) by hydrodynamic plasma motion.

Spectral shifting can occur by phase modulation caused by ionization and recombination or by plasma hydrodynamic motion through the Doppler effect. The hydrodynamic effect is dominant for dense, highly collisional plasmas [134, 135] while the phase modulation is seen mostly in subcritical, long-scale-length or gaseous plasmas [136]. We consider only hydrodynamic shifting.

The frequency shift is given by the Doppler formula

$$
\Delta \lambda=2 \lambda\left(v_{\exp } / c\right) \cos \theta
$$


where $\theta$ is the angle of incidence and $v_{\exp }$ is the plasma expansion speed. The expansion speed of a suddenly released semi-infinite fluid approximates our plasma and is given by

$$
v_{\exp }=\frac{2}{\gamma-1} c_{s}
$$

where $\gamma=C_{p} / C_{v}=1.6$ is the specific heat ratio and $c_{s}=Z T_{e} / m_{i}$ is the plasma sound speed. The expanding plasma scale length, $L=c_{s} \tau_{p}$, where $\tau_{p}$ is the pulsewidth, can also be calculated from spectral shift. Plasma expansion velocity and scale length as a function of temperature for the $\mathrm{Al}$ and $\mathrm{Au}$ targets are shown in Figure 4.8. The scale lengths for $\mathrm{Al}$ at $100-120 \mathrm{eV}$ and $\mathrm{Au}$ at $150-170 \mathrm{eV}$ are, $L / \lambda \approx 0.03$ and $L / \lambda \approx 0.02$ are in reasonable agreement with the scale lengths of $L / \lambda \approx 0.01$ determined from the Helmholtz wave equation model [114].

With the temperature-dependent scale length in hand, fractional resonance absorption of P-polarized light can be calculated. Resonance absorption is a laser-plasma coupling mechanism. The electric field of a Ppolarized light wave points along the density gradient as it refractively turns out of the plasma at the turning density $n_{c} \cos ^{2} \theta$. This field tunnels from the turning to the critical density. The evanescent field excites plasma oscillations at the critical surface where plasma and laser frequencies are in resonance. Electrostatic plasma oscillations leave the interaction region and are damped by collisional and collisionless processes, so energy is transferred from the laser field to the heated plasma. The absorption process is illustrated in Figure 4.9. The energy fraction absorbed by resonance absorption can be expressed as [96]

$$
f_{R A}=\frac{1}{2} \phi^{2}(\tau)
$$

where $\phi(\tau)$ describes plasma wave excitation strength in terms of incident angle and scale length by

$$
\phi(\tau)=2.3 \tau \exp \left(-2 \tau^{3} / 3\right), \quad \tau=(\omega \mathrm{L} / \mathrm{c})^{1 / 3} \sin \theta
$$



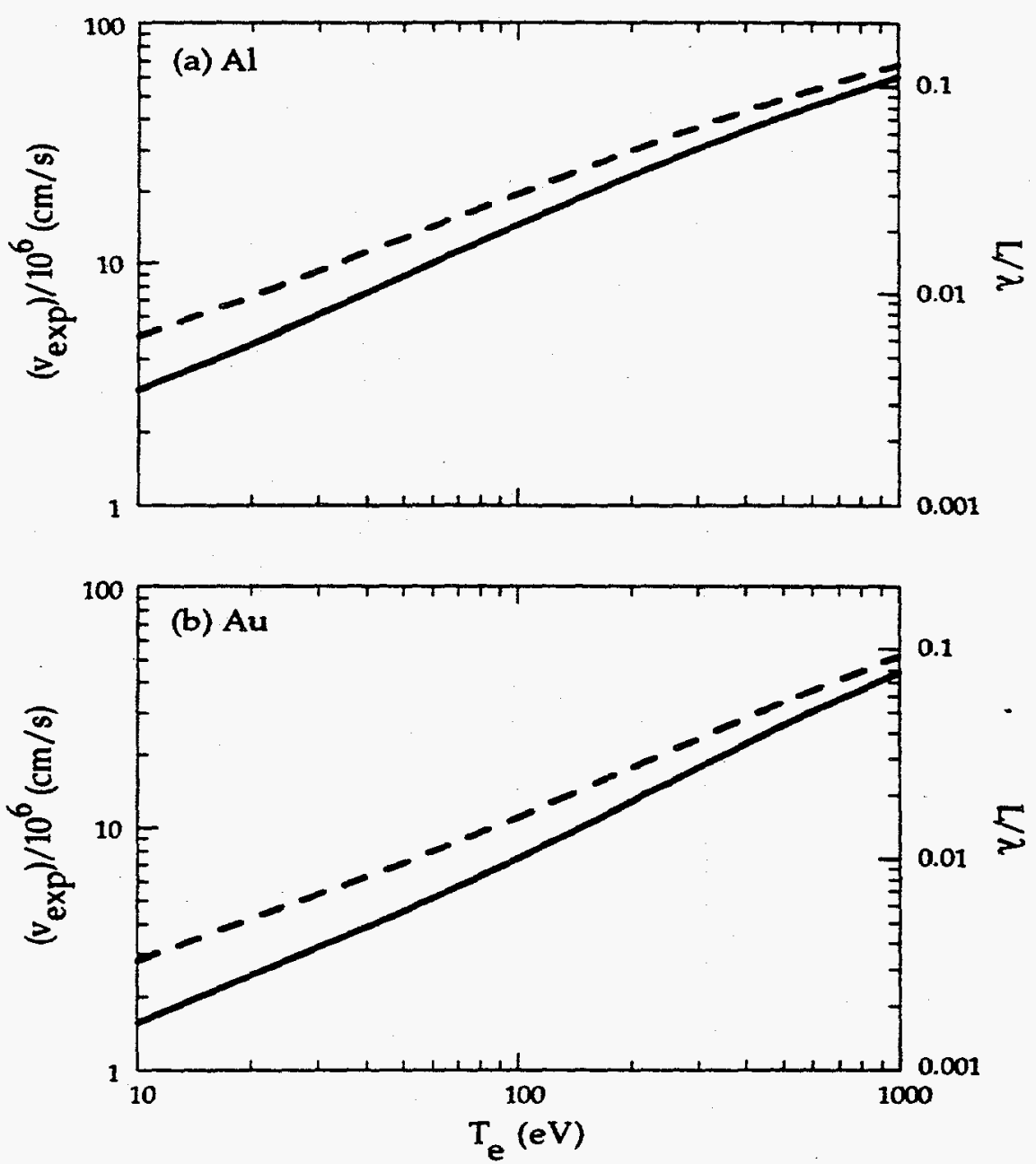

Figure 4.8: Calculated plasma expansion velocity (solid) and normalized scale length (dash) versus temperature for the (a) Al target and (b) Au target.

Using this expression, the percentage resonance absorption of a P-polarized wave was calculated as shown in Figure 4.6 above. Total percentage absorption (including linear inverse Bremsstrahlung absorption) is also shown in the figure. Total calculated absorption for $\mathrm{Al}$ is $27-29 \%$, in fair agreement with $35 \%$ observed absorption when scattered light is accounted for. Calculated total absorption for Au is $32-35 \%$, again in fair agreement with the observed $40 \%$ absorption. 


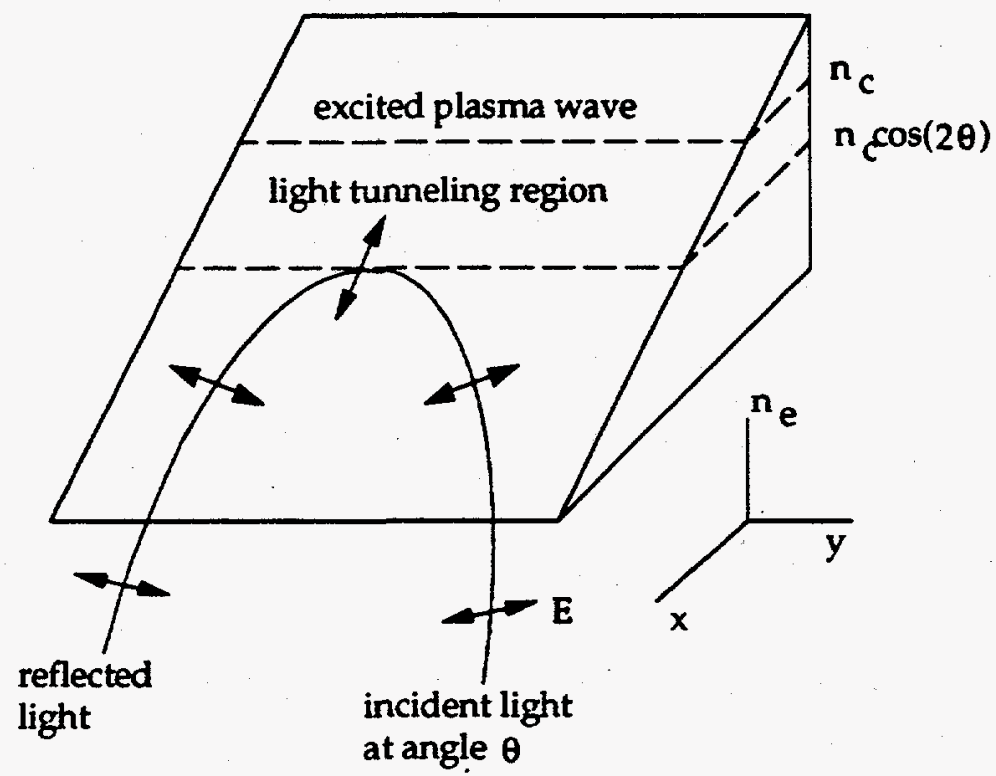

Figure 4.9: Illustration of resonance absorption occurring from the resonant coupling of P-polarized light to an electrostatic plasma wave at the critical density (after [94]).

Finally, we return to the temperature calculation by combining Eqs. (4.3) and (4.4) to obtain an expression to infer plasma temperature from spectral shift

$$
T_{c}=\frac{m_{i} c^{2}}{16 k Z}\left(\frac{\gamma-1}{\cos \theta}\right)^{2}\left(\frac{\Delta \lambda}{\lambda}\right)^{2}
$$

For example, the Al target's $3.3 \AA$ spectral shift, shown in Figure 4.7, gives a temperature of $126 \pm 4.6 \mathrm{eV}$ at $\sim 10^{15} \mathrm{~W} / \mathrm{cm}^{2}$, in good agreement with the $100-120 \mathrm{eV}$ temperature obtained from the reflectivity. The results of Figures $4.5,4.6$, and 4.8 are related to the laser intensity by the spectral shift measurement at several intensities. Al temperatures inferred from spectral shifting as a function of intensity and the $\mathrm{Al}$ computer simulation (LASNEX) calculations are compared in Figure 4.10. The LASNEX simulation is discussed in Section 4.3.3.4. Temperatures inferred by spectral shifting are somewhat higher than the simulation. This occurs because the spectral shifting predominantly measures lower-density, hotter expanding plasma. The LASNEX calculation is a better temperature estimate at the density of maximum absorption [134]. 


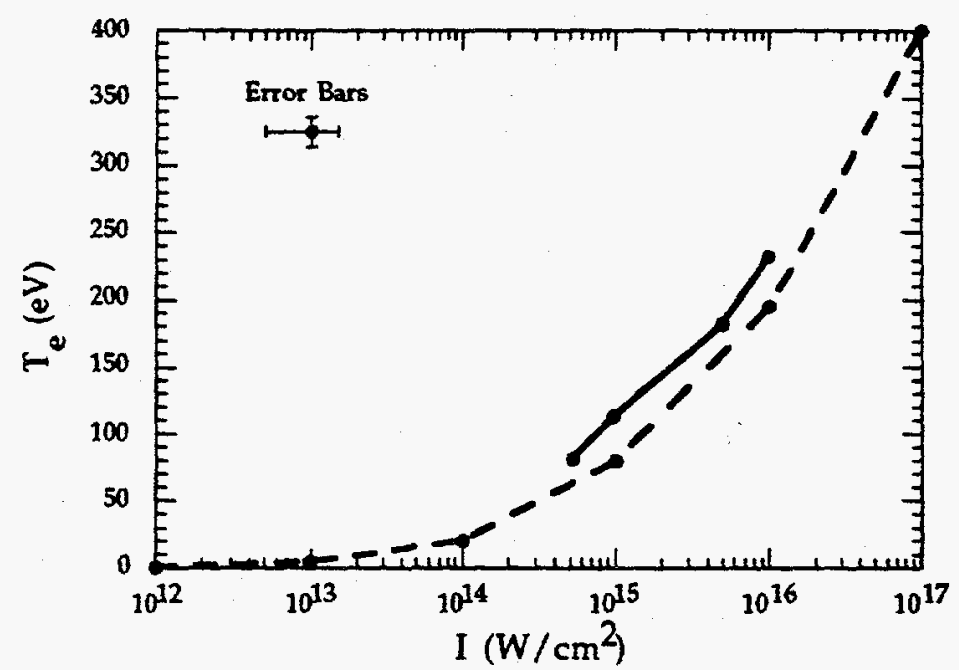

Figure 4.10: Inferred temperature versus measured intensity for the Al (solid) target. The temperature is inferred by measured reflected light spectral shifts. The calculated temperature for Al from the LASNEX simulation (dash) is also shown. Error bars indicate the $\pm 4.6 \mathrm{eV}$ due to the detector resolution limit $(0.4 A)$ for the spectral shift and estimated focused intensity uncertainty of $50 \%$.

\subsubsection{High-Field Effects}

A strong-field environment modifies absorption in several ways: collisional absorption is decreased by nonlinear inverse Bremsstrahlung effects, (2) noncollisional, vacuum heating occurs in steep-gradient plasmas, and (3) pondermotive pressures steepen the electron density profile, decreasing the inverse Bremsstrahlung absorption volume.

The critical parameter for nonlinear inverse Bremsstrahlung effects is the ratio of the quiver velocity, $v_{o s c}=e E / m \omega$, of a free electron in the field to the electron thermal velocity, $v_{t h}=\left(T_{e} / m_{e}\right)^{1 / 2}$

$$
\frac{v_{o s c}}{v_{t h}}=\left(\frac{I}{10^{16} \mathrm{~W} / \mathrm{cm}^{2}}\right)^{1 / 2}\left(\frac{\lambda}{249 \mathrm{~nm}}\right)\left(\frac{200 \mathrm{eV}}{T_{e}}\right)^{1 / 2}
$$


When $v_{\text {oxc }} \sim v_{t h}$, collisional absorption is reduced below its low field value. The collision rate depends on the local field value that, as mentioned previously, is shielded inside the critical surface by the factor $\omega / \omega_{p}$. We calculate reflectivity based on an average shielded field near the highest intensity $\left(5 \times 10^{15} \mathrm{~W} / \mathrm{cm}^{2}\right)$.

Two principle mechanisms are responsible for reduced inverse Bremsstrahlung absorption. First, the electron-ion collision frequency is modified by the electron's coherent oscillatory motion in the laser field. Effective velocity under the field's influence is expressed as

$$
v_{f f f}^{2}=v_{t h}^{2}+v_{o s c}^{2}
$$

Schlessinger and Wright [101] have shown that an approximate correction factor, $F_{1}$, to the collision rate is

$$
F_{1}=\left(1+\left\langle v_{o s c}^{2}\right\rangle / 3 v_{t h}^{2}\right)^{-3 / 2}
$$

Using this factor, we calculated the high-intensity percentage absorption to be reduced by the factor $F_{1}$ to the values shown in Table 4.1.

The second nonlinear inverse Bremsstrahlung absorption effect occurs because the electron velocity distribution function can be driven far from a Maxwellian in high fields [100]. The rate at which an electron gains energy from the field is proportional to $v_{e-i} v_{o s c}^{2}$, where $v_{e-i}$ is the electron-ion collision frequency, and the rate at which the electron can distribute this energy to form a Maxwellian is $v_{e-e} v_{t h}^{2}$, where $v_{e-e}=v_{e-i} / Z$ is the electron-electron collision rate. Electrons therefore gain energy from the laser faster than they can form a Maxwellian when

$$
Z\left(\frac{\left\langle v_{\text {osc }}\right\rangle}{v_{\text {th }}}\right)^{2}>1
$$

The absorption decrease from a modified electron distribution can be approximated by the factor, $F_{2}$ 


$$
F_{2}=1-0.553\left[1+\left(\frac{0.27 v_{t h}^{2}}{Z\left(v_{o x c}^{2}\right\rangle}\right)^{0.75}\right]^{-1}
$$

Using this factor, we calculated the high-intensity percentage absorption to be reduced by the factor $F_{2}$ to the values shown in Table 4.1.

Vacuum heating arises when an obliquely incident, P-polarized field drags electrons from the plasma to the vacuum and then drives them back into the plasma at high velocities. Vacuum heating is more efficient than resonance absorption (Section 4.3.3.1) when $v_{\text {osc }} / \omega>L$ [124], where $\omega$ is the laser frequency, $v_{\text {osc }}=e E / m_{e} \omega$ is the electron quiver velocity, and $L$ is the density gradient scale length. This condition comes from a consideration of the length necessary to shield the electrostatic field of resonant absorption [124]. At our chosen high intensity $\left(5 \times 10^{15} \mathrm{~W} / \mathrm{cm}^{2}\right)$ and for $L / \lambda \approx 0.01$ this condition is satisfied. Gibbon and Bell [125] studied the phenomena of resonance absorption, vacuum heating and skin effects in a single particle-in-cell model. At long scale lengths, $L / \lambda \gg 0.1$, they observed resonant absorption. As the scale length was reduced, a transition to vacuum heating was seen. For $L / \lambda<0.1$ vacuum heating was responsible for most of the P-polarized absorption with a maximum efficiency at $v_{o s c} / c=3.1(L / \lambda)^{2}$. Calculated vacuum heating absorption at $5 \times 10^{15} \mathrm{~W} / \mathrm{cm}^{2}$ and $L / \lambda \approx 0.01$ is approximately $10 \pm 2 \%$ [125]. This figure is added to the linear and nonlinear inverse Bremsstrahlung, and resonance absorption in Table 4.1 to arrive at the net absorption for P-polarization. Gibbon also found that at zero scale length, vacuum heating vanished and anomalous skin effects became important [125].

Net absorption from linear and nonlinear inverse Bremsstrahlung ( $\mathrm{S}$ and $\mathrm{P}$ polarization), resonance absorption ( $P$ polarization), and vacuum heating ( $P$ polarization) is about $15 \%$ below its observed values. The discrepancy may be 
accounted for in two ways. First, Kruer's estimate of linear inverse Bremsstrahlung absorption is based on a low collisionality plasma $\left(v_{e-i} / \omega \ll 1\right)$ WKB approximation that is strictly valid only for gentle, i.e., long pulse, density gradients. In particular, the solution begins to break down near the turning point density, where most short-pulse absorption occurs. Second, a significant fraction of the incident light is scattered diffusely and not reflected specularly. The experiment measured only specularly reflected light. Fedosejevs [114] measured this diffuse fraction from an $\mathrm{Al}$ target to be $-5 \pm 2 \%$ for intensities of $1014 \mathrm{~W} / \mathrm{cm}^{2}$ rising to about $13 \pm 2 \%$, in the regime calculated here with intensities $>1015 \mathrm{~W} / \mathrm{cm}^{2}$ and an angle $\sim 15^{\circ}$. When the $13 \pm 2 \%$ scattered light is added to the net absorption, absorption calculations are in very good agreement with the observed values at the highest intensities. Considering these results, $\mathrm{Al}$ and $\mathrm{Au}$ plasma reflectivities for intensities $\sim 10^{16} \mathrm{~W} / \mathrm{cm}^{2}$ are well described by linear and nonlinear inverse Bremsstrahlung absorption ( $S, P$ polarization), resonance absorption ( $\mathrm{P}$ polarization), and vacuum heating ( $\mathrm{P}$ polarization).

A final consideration is pondermotive steepening. Pondermotive steepening is a complex process which interacts self-consistently with field enhancement and enhanced ionization. These are all time-dependent effects that require a model beyond the scope of this work. The electron density profile can be steepened by the high-field pondermotive force. The gradient in the field pressure causes the pondermotive force $F_{p}$

$$
\mathbf{F}_{p}=-\frac{e^{2}}{4 m_{e} \omega^{2}} \nabla \mathbf{E}^{2}(\mathbf{x})
$$

which pushes plasma away from regions of high field pressure and steepens the density profile. Simulations $[122,137]$ have shown an initially smooth, linear density profile develops a sharp step near critical density. Since most inverse 


\begin{tabular}{ccccccc}
\hline & & Abs. (\%) & Abs. (\%) & Abs. (\%) & Net Abs. + Obs. Abs. + \\
Material & Pol'n & Linear IB & $\left(F_{1}\right)$ & $\left(F_{2}\right)$ & Scatter (\%) & Scatter (\%) \\
\hline Al & S & $21-23$ & $17-19$ & $11-12$ & $24-25 \pm 4$ & $25 \pm 5$ \\
Al & P & $27-29$ & $23-26$ & $14-15$ & $36-38 \pm 4$ & $35 \pm 5$ \\
Au & S & $28-30$ & $26-28$ & $14-16$ & $28-30 \pm 4$ & $30 \pm 5$ \\
Au & P & $32-35$ & $30-33$ & $17-18$ & $40-42 \pm 4$ & $40 \pm 5$ \\
\hline
\end{tabular}

Table 4.1: Calculated and obseroed values of percentage absorption for Al at 100-120eV and Au at 150$170 \mathrm{eV}$. Linear inverse Bremsstrahlung does not account for intensity-dependent collisions. The factors $F_{1}$ and $F_{2}$ account for intensity-dependent modifications to the electron trajectory and distribution, respectively. Net absorption is the product of both nonlinear absorption reduction factors, $F_{1}$ and $F_{2}$, and the percentage linear inverse Bremsstrahlung absorption. Net absorption for P-polarization includes resonance absorption and 10\% vacuum heating. Uncertainty in the observed value is taken to be $\pm 5 \%$ corresponding to the scatter in the data. Uncertainty in oacuum heating and scatter percentage estimates is about $\pm 2 \%$ each. All calculations are rounded to the nearest integer oalue.

Bremsstrahlung absorption occurs at densities near critical, profile steepening can eliminate some of the region where absorption is strongest [138] and thus reduce absorption. The threshold for pondermotive steepening is [134]

$$
\frac{I\left[\mathrm{~W} / \mathrm{cm}^{2}\right] \lambda^{2}[\mu \mathrm{m}]}{T_{\mathrm{e}}[\mathrm{eV}]}=3 \times 10^{12} \mathrm{~W} \mu \mathrm{m}^{2} / \mathrm{eVcm}
$$

Calculated values are $1.4 \times 10^{12} \mathrm{~W} / \mathrm{cm}^{2}(\mathrm{Al})$ and $6 \times 10^{11} \mathrm{~W} / \mathrm{cm}^{2}(\mathrm{Au})$, which are close enough to threshold that the effect should not be ignored. Besides the intensity, the time for the steepening to develop must be considered. Rae [98] describes how the steepening occurs much more slowly than the pulse interaction time for S-polarized light. For P-polarized light, however, movement across the narrow critical region can occur much faster, because a large electric field enhancement occurs near critical density [114]. Thus, pondermotive steepening may explain why P-polarized calculations slightly overestimate the values in Table 4.1, even though S-polarization calculations slightly 
underestimate the data. Note that the enhanced field at the critical density will also increase ionization which further affects absorption.

\subsubsection{Strongly-Coupled Plasma Effects}

Resistivity as a function of temperature of nearly unexpanded metal plasmas can be calculated, but results are sensitive to the exact collision frequency form [139]. Determination of the collision frequency form is beyond the scope of this work. It is critically dependent on inclusion of both high-field and strongly-coupled plasma effects.

What is a strongly-coupled plasma? A measurement of coupling strength is the ratio of the average Coulomb energy to the average kinetic energy and can be expressed as [140]

$$
\Gamma=2.69 \times 10^{-5} Z^{2}\left(\frac{n_{e}}{10^{12} \mathrm{~cm}^{-3}}\right)^{1 / 3}\left(\frac{10^{6} \mathrm{~K}}{T_{e}}\right)
$$

Plasmas with $\Gamma>1$ are termed strongly-coupled. Common laboratory plasmas are weakly-coupled, $\Gamma \ll 1$. Strong coupling examples are typically stellar or planetary interiors, however, inertial confinement fusion, shock tube, and ultrashort pulse plasmas can also reach strongly-coupled conditions. The coupling parameter as a function of density and temperature for the $\mathrm{Al}$ and $\mathrm{Au}$ targets is shown in Figure 4.11. 


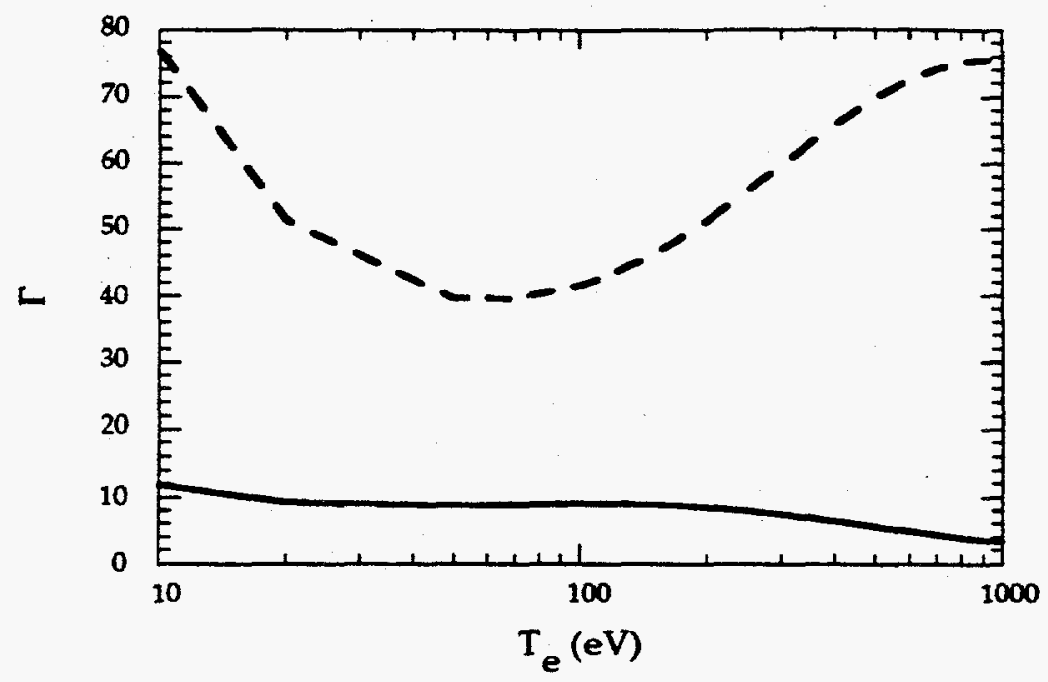

Figure 4.11: The coupling parameter versus temperature for $A l$ (solid) and Au (dash).

Plasma density-temperature plots are shown in Figure 4.12 to relate our calculated coupling parameter to various plasma regimes. Region 0 in Figure 4.12 is the classical plasma region. Here Debye-Huckel theory describes screening of long-range Coulomb interactions. Screening is a long-range effect where many particles are involved and contribute to overall transport coefficients. Region 1 is the dense plasma regime. In dense plasmas, the Debye length is about the atomic spacing, so ion pair interactions are strong. Rydberg states bound to adjacent ions interact and form conduction bands that lower the boundary between bound and free states (continuum lowering). Region 2 is described as high-density matter. Matter is compressed above solid density. Free electrons are partly or completely degenerate. Ions are packed so tightly that they exert pressure on each other so even occupied inner-shell states form energy bands (pressure ionization). 

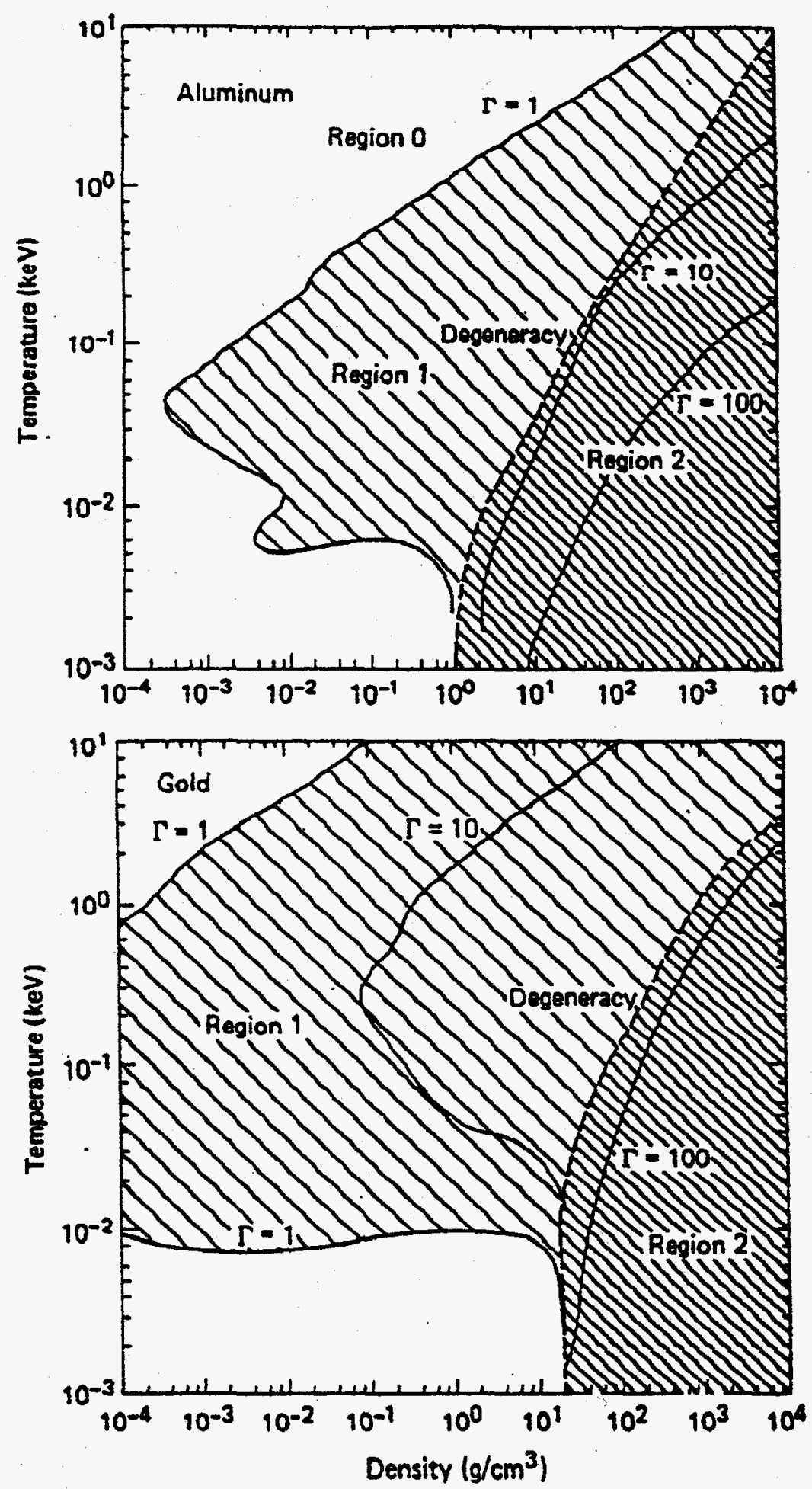

Figure 4.12: The plasma coupling parameter as a function of density and temperature for $A l$ and $A u$. (after [133]) 
A number of authors [139, 141-145] have proposed detailed models to simulate experimentally inferred resistivity of $\mathrm{Al}$ heated by intense, ultra-short pulses [3]. The models have had limited success at explaining the measurement [139]. We justify our Spitzer absorption treatment above, because it shows the correct high-temperature behavior. Moreover, the absolute high-intensity absorption is not as sensitive to plasma conditions as the functional form of resistivity over a broad temperature and density range.

\subsubsection{Numerical Simulation}

The $\mathrm{Al}, \mathrm{S}$-polarization reflectivity result is also compared over the entire intensity range to a one dimensional plasma code (LASNEX) [146] in Figure 4.13. LASNEX treats plasma with hydrodynamic fluid equations and is well described by Alley [147] and Landen [134]. The inputs were $614 \mathrm{~nm}$, S-polarized, $150 \mathrm{fs}$ (FWHM) sech ${ }^{2}$ pulses at $15^{\circ}$ incidence angle with peak intensities from $10^{12}$ to $10^{16} \mathrm{~W} / \mathrm{cm}^{2}$. Reflectivity results show good quantitative agreement only at high intensities. Qualitatively, the simulation appears to find a reflectivity minimum near the shallow minimum in the data that corresponds to the resistivity saturation discussed in Section 4.3.1. LASNEX produces poor low intensity results because, as mentioned in Section 4.3.3.3, the detailed form of the conductivity is not well known for the transition region between cold solid and hot plasma. The LASNEX code is designed for inertial confinement fusion calculations at temperatures well above this transition region. The quality of fit is comparable with other detailed simulations [139]. 


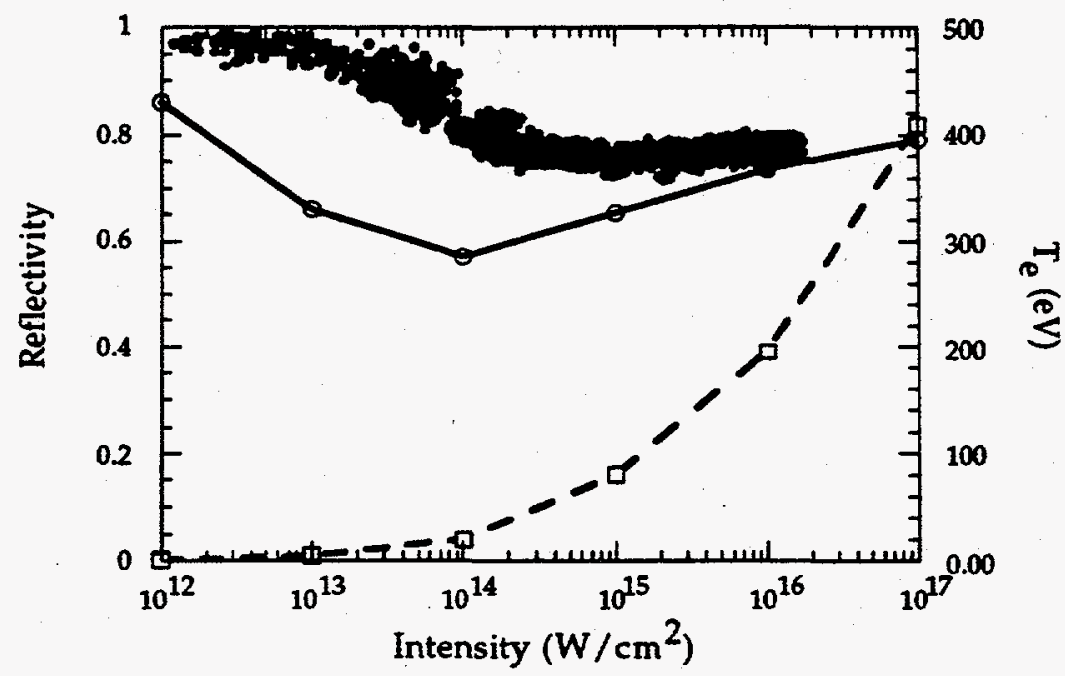

Figure 4.13: Simulation results from the LASNEX code for Al reflectioity (solid) and peak electron temperature (dash) compared to the reflectivity data (solid circles).

\subsubsection{Analysis of Results: The Insulator $\mathrm{SiO}_{2}$}

Because insulators begin with virtually no free electrons, they represent an interesting case to understand and simulate. During plasma formation, electron density changes over twenty-two orders of magnitude while laser intensity changes over sixteen orders of magnitude. Both changes occur on a femtosecond timescale according to highly nonlinear processes (avalanche or tunneling) which are sensitive to the initial density and temperature state. Clearly this is a problem that demands careful consideration. We do not attempt to explain the physics by the simple absorption treatment used in previous sections for metals. In addition, none of the strongly-coupled models referenced above were previously developed for insulators, so they cannot be used.

It is well known that at low intensities ultra-short pulses can create nonequilibrium temperature distributions between the electrons and the lattice [148-150]. If the pulse intensity is increased by several orders of magnitude, one 
might expect nonequilibrium ionization conditions could exist. Using this idea, a model is proposed in Section 4.4 that relies entirely on rate equations and lets the ionization be determined by the ionizing process and not by an assumed plasma model such as LTE.

\subsection{A NONEQUILIBRIUM PLASMA MODEL FOR INSULATORS}

\subsubsection{Basic Equations}

It is assumed that a solid can be approximated as a collection of independent atoms and solid ionization and recombination cross sections are similar to their gaseous counterparts. Coulomb ionization potential lowering, a solid state effect, is taken into account by parameterizing the atomic cross section with the solid ionization potential. It is also initially assumed an atom is at most singly ionized. This starting approximation underestimates electron density, but does not significantly reduce self-reflection (for visible wavelength lasers). When every atom in a typical solid is only singly ionized, electron density is several times larger than the critical density needed for self-reflection. Further contributions to electron density from multiple ionization beyond this supercritical density has a small effect on self-reflectivity, as will be shown in Section 4.4.8. Single ionization allows physically intuitive, closed form results to be obtained.

The equation for net production of electrons per unit volume, $n_{e}$, is

$$
\frac{d n_{e}}{d t}=\alpha\left(N_{0}-n_{e}\right) n_{e}-\rho n_{e}^{3}+\omega_{t}\left(N_{0}-n_{e}\right)
$$

where $\alpha, \rho$, and $\omega_{t}$ are, respectively, the coefficients for collisional ionization, three-body recombination and strong-field laser tunneling ionization, and $N_{0}$ represents the initial density of atoms. Photorecombination is neglected altogether, because it is small compared to three-body recombination unless the density is $n_{e}<3.1 \times 10^{13} T_{e}^{3.75} / \mathrm{Z} \sim 10^{20}-10^{21} \mathrm{~cm}^{-3}$. [151]. 
$\left(N_{0}-n_{e}\right)$ is an important term in Eq. (4.16), because it determines electron density saturation. $\left(N_{0}-n_{e}\right)$ is the time-dependent density of atoms available for ionization as plasma is being created. Because of particle conservation, the density of unionized atoms decreases as the electron density increases as described by this factor. This reduction of atoms causes the electron density to saturate. A complete derivation of Eq. (4.16) as well as electron density rate equations which include multiple ionization are given in Appendix A.

A single ionization model is initially used, because we can obtain analytic solutions and scaling relations. We cannot derive closed form solutions for the more general case of multiple ionization. In Section 4.4.8, a numerical simulation including multiple ionization is compared with single ionization results. The results agree within $5 \%$.

The average total electron energy density, $E^{\prime}$, is given by the equation of state $E^{\prime}=(3 / 2) n_{e} k T_{e}-\left(N_{0}-n_{e}\right) I_{p}$, where $T_{e}$ is the electron temperature, $k$ is the Boltzmann constant, and $I_{p}$ is the ionization potential. The first term represents the average free electron kinetic energy density, while the second represents the bound electron potential energy density. Kinetic energy is equated to an electron temperature, $T_{e}$, to relate energy deposition to collisional ionization and threebody recombination processes of Eq. (4.16). Note that although this section deals with a nonequilibrium situation, a temperature is used. Our temperature is a parameter for average electron kinetic energy as discussed in Zel'dovich and Raizer [56]. The constant term $N_{0} I_{p}$ can be added to the total energy density without affecting plasma formation or energy deposition. The sum $E^{\prime}+N_{0} I_{p}$ is the total energy density $E$ relative to the reference state $N_{0} I_{p}$, so the working equation of state is

$$
E=\frac{3}{2} n_{e} k T_{e}+n_{e} I_{p}
$$


We assume the net rate of energy gain by electrons in a unit volume is described by $d E / d t=k_{I B} I_{L}-(3 / 2) n_{e} k\left(T_{e}-T_{a}\right) \nu_{c}$. Here, $k_{I B}$ is the laser energy absorption coefficient, $I_{L}$ is the laser intensity, $T_{a}$ is the atomic temperature, and $v_{c}$ is the elastic electron-atom collision frequency. The first term represents energy absorbed from the laser by inverse Bremsstrahlung, while the second represents energy lost to heavy particles through collisions [152, 153]. Solving for $(3 / 2) n_{e} k T_{e}$ in Eq. (4.17) and substituting the result into this equation gives

$$
\frac{d E}{d t}=k_{I B} I_{L}+v_{c}\left(\frac{3}{2} n_{e} k T_{a}+n_{e} I_{p}-E\right)
$$

\subsubsection{Kinetic Coefficients}

For completeness, we discuss briefly the kinetic coefficients of Eqs. (4.16) and (4.18). The impact ionization coefficient $\alpha$ derived by Zel'dovich and Raizer $[151]$ is

$$
\alpha_{Z R}=8 \sqrt{\pi} \frac{\omega_{a u}}{n_{a u}} \beta^{3 / 2}\left[\frac{\exp \left(-\beta E_{p}\right)}{\beta E_{p}}-E_{1}\left(\beta E_{p}\right)\right]
$$

where the atomic unit of frequency $\omega_{a u}$ is equal to $4.1 \times 10^{16} \mathrm{sec}^{-1}$, the atomic unit of volume $1 / n_{a x}$ is equal to the cube of the Bohr radius $a_{0}{ }^{3}$, the dimensionless ionization potential $E_{p}$ is equal to $I_{p} / I_{H}$, the ratio of the atomic ionization potential, $I_{p}$, to the hydrogen ionization potential, $I_{H}$, the dimensionless inverse temperature $\beta$ is equal to $I_{H} / k T_{e}$, and $E_{1}$ is the exponential integral. This formula shows that impact ionization is a decreasing function of temperature when the temperature is a few times larger than the ionization potential. This high temperature behavior is the reason the primary ionization process switches from impact ionization to photo-ionization as laser intensity increases. In the high-intensity regime, electron temperature rises very rapidly, forcing the impact ionization rate well below the photo-ionization rate in the pulse's leading edge. 
Eq. (4.19) is a field-free result. In Appendix B a formula is derived for electron impact ionization which includes a change in the electron distribution caused by a strong field. Our result agrees with Eq. (4.19) at low-fields. The high-field formula is used in our calculations, but results differ from Eq. (4.19) by less than $1 \%$.

The recombination coefficient $\rho$ is related to $\alpha$ through the principle of detailed balance, and its formula is

$$
\rho=\alpha \frac{(4 \pi)^{3 / 2}}{2} \frac{1}{n_{a u}} \beta^{3 / 2} \exp \left(\beta E_{p}\right)
$$

The strong-field ionization rate $\omega_{t}$ is approximated by the tunneling ionization rate in a hydrogenic atom by a static field [154]

$$
\omega_{\mathrm{t}}=4 \omega_{a x} \frac{E_{p}^{5 / 2}}{F} \exp \left(-\frac{\frac{2}{3} E_{p}^{3 / 2}}{F}\right)
$$

$F$ is the dimensionless laser field strength $27.46\left(I_{L}\left(\mathrm{~W} / \mathrm{cm}^{2}\right)\right)^{3 / 2} / 5.1 \times 10^{9} \mathrm{~V} / \mathrm{cm}$.

Researchers studying laser ionization of gases in the strong field regime find this formula to give reliable fits to their data for non-hydrogenic atoms [155].

The inverse Bremsstrahlung deposition coefficient $k_{I B}$ in $\mathrm{cm}^{-1}$ is equal to Spitzer's rate [115] divided by $c$ the speed of light, i.e., $c k_{18}=2 \pi \gamma$. It is

$$
k_{I B}=\frac{16}{3} \sqrt{\frac{\pi}{3}}\left(\frac{2 \pi e^{2}}{h c}\right)^{3} n_{e} \lambda^{2} \frac{N_{0}}{n_{a u}} \beta^{3 / 2}
$$

A correction factor [42] can be used to account for decreased inverse Bremsstrahlung heating due to the high laser intensity. It is not considered in Eq. (4.22), because we want to test the tunneling rate against the maximum possible collision rate. The cooling rate $v_{c}$ in $\sec ^{-1}$ is also Spitzer's [115]

$$
v_{c}=\frac{32}{3} \sqrt{\pi} \omega_{a u} \beta^{3 / 2} \frac{N_{0}}{n_{a u}} \frac{m_{e}}{m_{a}}\left[1+\frac{m_{e} T_{e}}{m_{a} T_{a}}\right]^{3 / 2}
$$

where $m_{a}$ is the atomic mass. 


\subsubsection{Numerical Results for $n_{e}$ and $T_{e}$}

Numerical solutions for electron density and temperature exhibit nonequilibrium plasma formation dynamics during self-reflection of an intense laser pulse from a transparent material. To simulate quartz ionization, $I_{p}$ is set equal to $9 \mathrm{eV}$ to represent the bandgap of quartz, $N_{0}$ is equated to $10^{22} \mathrm{~cm}^{-3}$ to represent approximate atomic density, the atomic temperature, $T_{a}$, is fixed at $.025 \mathrm{eV}, n_{e}$ is given the initial value of zero $\mathrm{cm}^{-3}$, and $T_{e}$ is given an initial value of $.025 \mathrm{eV}$. Initializing electron temperature to a non-zero value avoids the temperature singularity of $k_{I B}$ in Eq. (4.22). This approximation is insignificant, since the temperature singularity is integrable, as shown in Section 4.4.6. We have also found our results to be insensitive to small $T_{e}$ variations near $.025 \mathrm{eV}$. This insensitivity arises because the temperature singularity of $k_{I B}$ is mitigated by the initialization of $n_{e}$ to zero.

Numerical results obtained for peak laser intensities of $10^{14} \mathrm{~W} / \mathrm{cm}^{2}$ and $10^{16} \mathrm{~W} / \mathrm{cm}^{2}$ are shown in Figures 4.14 (a)-(b), respectively. The laser pulse, $I_{\text {peat }} \operatorname{sech}^{2}\left(1.76\left(t-t_{\text {patk }}\right) / \tau\right)$ with $t_{\text {peat }}=375 \mathrm{fs}$ and $\tau=125 \mathrm{fs}$, is superimposed on these figures to give a time reference to the ionization dynamics. A sech function is used, because it shows excellent agreement with the pulseshape derived from a second order amplified laser pulse autocorrelation. 

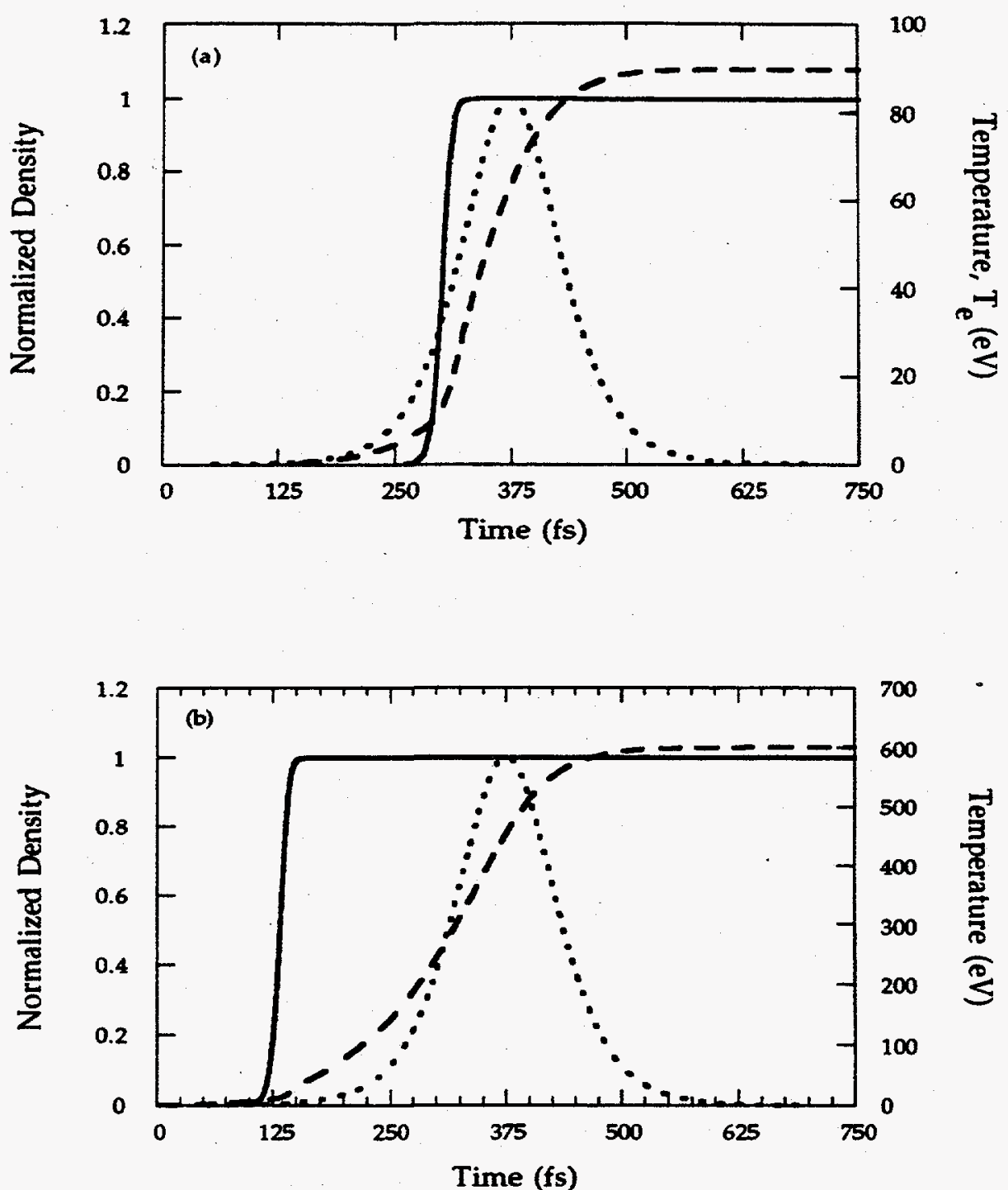

Figure 4.14: (a) The electron density $10^{22} \mathrm{~cm}^{-3}$ (solid), electron temperature (dash) and pulse intensity/10 $0^{14} \mathrm{~W} / \mathrm{cm}^{2}$ (dot) are shown as functions of time. Electron density falls slightly at the pulse's tail end because electrons are able to recombine at low temperatures $(<50 \mathrm{eV})$. (b) Peak intensity is increased to $10^{16} \mathrm{~W} / \mathrm{cm}^{2}$. Electron density/1022 $\mathrm{cm}^{-3}$ (solid), electron temperature (dash), and pulse intensity $10^{16} \mathrm{~W} / \mathrm{cm}^{2}$ (dot) are shown as functions of time. Note the temperature is now too high for significant recombination to occur during the pulse interaction time. 
These figures show $n_{e}$ rises to the constant value of $N_{0}$. This electron density saturation is caused by the $N_{0}-n_{e}$ factor of Eq. (4.16). We also conclude $n_{e}$ is not affected by recombination for at least a few hundred femtoseconds after the pulse's peak, since the final portion of $n_{e}$ decreases only slightly. This insensitivity of plasma formation to recombination is demonstrated in Figure 4.15. Comparing $n_{e}$ obtained by setting $\rho$ equal to zero to $n_{e}$ obtained for $\rho$ determined from Eq. (4.20) shows the two cases to be virtually identical. This conclusion is also drawn by inspecting Eq. (4.20). In order for $\rho n_{e}$ to be of the same order as $\alpha, n_{e}$ must be larger than $5.5 \times 10^{24} \mathrm{~cm}^{-3}\left(\right.$ at $\left.T_{e}=100 \mathrm{eV}\right)$, which is over 500 times larger than its maximum value assuming single ionization.

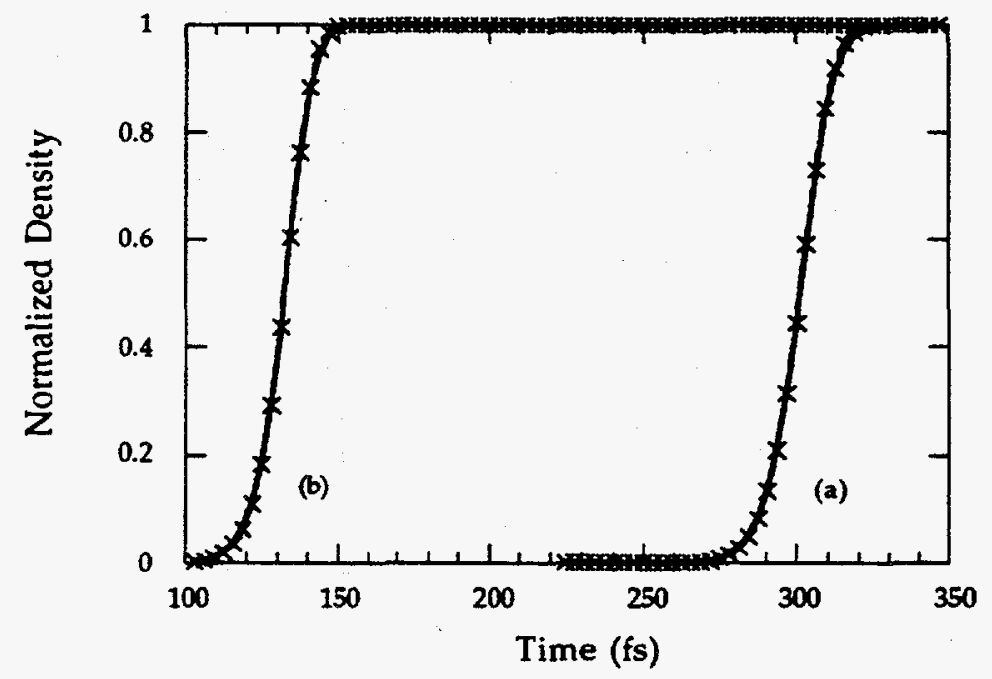

Figure 4.15: (a) Comparison of density shown in Figure 4.14 (a) (solid) and density calculated without recombination $(X)$ shows insignificant recombination on pulse's rising edge at $10^{14} \mathrm{~W} / \mathrm{cm}^{2}$, because the electron density is low. Recombination is also unimportant on the falling edge, because the temperature, $T_{e}>80 \mathrm{eV}$ is too high for significant recombination. Hence, recombination has little effect on electron population for intensities greater than $-10^{14} \mathrm{~W} / \mathrm{cm}^{2}$. (b) the same as (a), but the peak pulse intensity is $10^{16} \mathrm{~W} / \mathrm{cm}^{2}$. Recombination becomes less important as the peak intensity increases, because a higher intensity drives the temperature up. 
At high intensities and small pulse widths, the majority of electrons are produced by strong field electron tunneling ionization. Avalanche and tunneling ionization rates as a function of intensity are shown in Figure 4.16. The preponderance of tunneling electrons is demonstrated in Figure 4.17 by comparing the density of electrons produced from tunneling ionization alone to the density produced with avalanche ionization included. In both cases, recombination is neglected. Figure 4.17 shows a negligible electron density contribution from avalanche ionization. Therefore, at intensities greater than $10^{14} \mathrm{~W} / \mathrm{cm}^{2}$, initial plasma formation is field-driven photo-ionization, not temperature-driven avalanche ionization. We return to this point in Section 4.4.7 where we show the plasma is in a transient NLTE state, because tunneling ionization forms high ion states faster than collisions can equilibrate them to a Saha distribution.

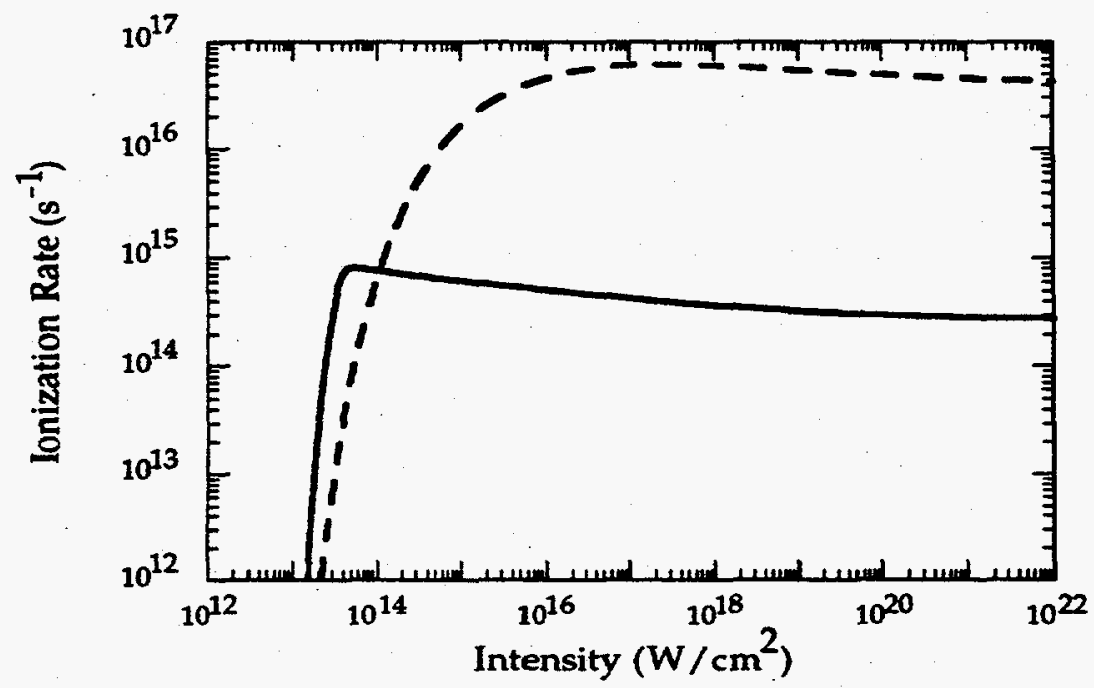

Figure 4.16: Comparison of avalanche (solid) and tunneling (dash) ionization rates versus laser intensity. 


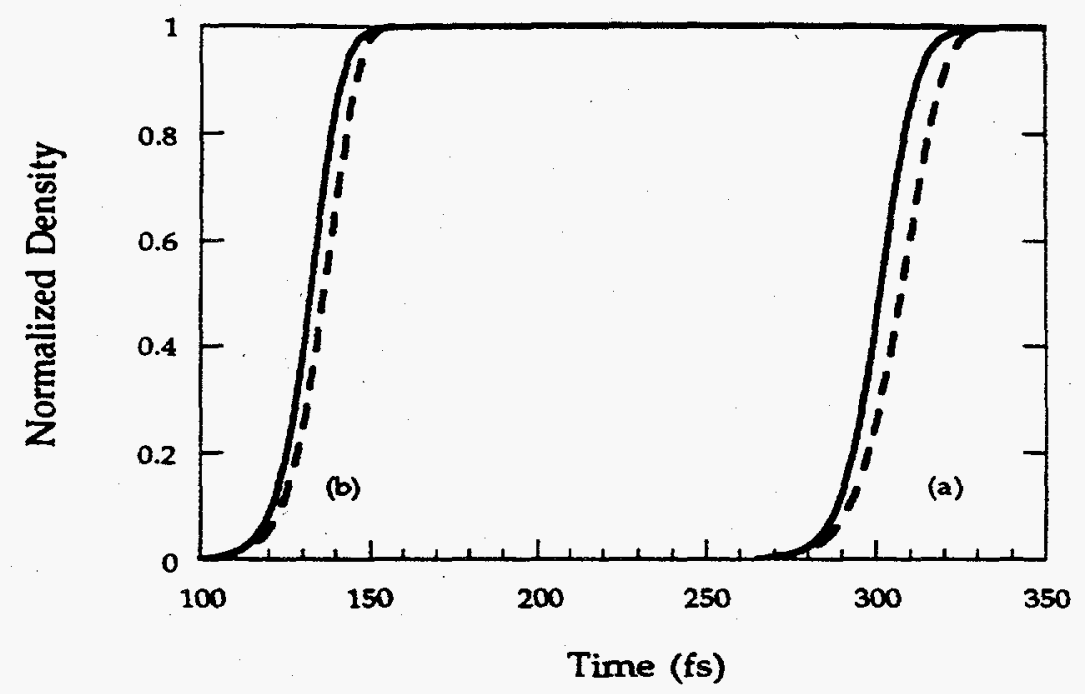

Figure 4.17: (a) This comparison of Figure 4.14 (a) (solid) and the case $\alpha=0$ (dash) shows that aoalanche ionization is unimportant compared to photoionization for intensities as low as $10^{14} \mathrm{~W} / \mathrm{cm}^{2}$. (b) The same as in (a), but with a peak pulse intensity of $10^{16} \mathrm{~W} / \mathrm{cm}^{2}$. Avalanche ionization becomes even less important at higher intensities.

Since dominance of tunneling over impact ionization is of key importance to our model, it is worthwhile to digress and note agreement with other authors. Braunlich [156] found that in the pre-breakdown regime, virtually all lattice heating, up to melting, occurs via nonlinear absorption of laser photons by multiphoton excited free electrons (free-electron heating [157]). A two-color experiment [157] allowed independent control of starting electron density and lattice heating and demonstrated that free-electron heating efficiently transfers energy from the laser field to the lattice. Moreover, these results have been confirmed by Monte Carlo simulations [158]. These and other recent works [157] have identified an avalanche suppression mechanism as strong free electron scattering by non-polar acoustic phonons through the acoustic deformation potential interaction $[14,159,160]$. This scattering prevents free carriers from reaching energies high enough to cause impact ionization and initiate avalanche 
breakdown before multiphoton ionization takes over. Electron-phonon scattering rates in $\mathrm{SiO}_{2}$ as a function of electron energy are shown in Figure 4.18. The polar phonon interaction is strongest at the energy of longitudinal optical (LO) phonon modes [161] and decreases at higher energies. However, the scattering rates for non-polar collisions with acoustic (TA and LA) and transverse-optical (TO) phonons grow with increasing electron energy. Thus, a heated electron distribution is stabilized against runaway velocity energies so avalanche breakdown is held off. This important departure from standard avalanche theory is used here, for the first time, to predict laser damage (plasma formation) characteristics in the ultra-short pulse regime.

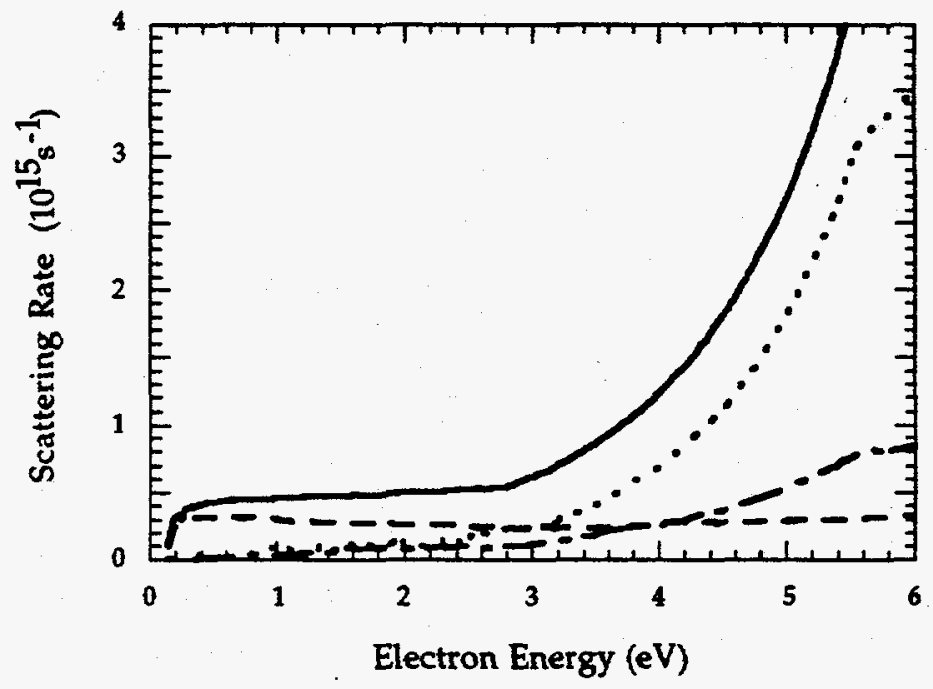

Figure 4.18: Total (solid), polar (dash), nonpolar TA, LA (dot), and nonpolar TO (dash-dot) electron-phonon scattering rates in $\mathrm{SiO}_{2}$ versus electron energy (after [156]).

The question remains whether the avalanche can be suppressed for higher fields than those considered by Braunlich $\left(\sim 10^{6} \mathrm{~V} / \mathrm{cm}\right)$. At some energy, the nonpolar scattering rate must decrease because the cross-section will begin to decrease with increasing electron energy. Bloembergen [14] extrapolated data to 
shorter pulsewidths to show that for pulses $<10^{-12} \mathrm{~s}$ the avalanche threshold would exceed $2 \times 10^{7} \mathrm{~V} / \mathrm{cm}$ corresponding to an intensity of $10^{12} \mathrm{~W} / \mathrm{cm}^{2}$. Extrapolating to $\sim 10^{-13} \mathrm{~s}$ pulses, the avalanche threshold is $\sim 10^{13} \mathrm{~W} / \mathrm{cm}^{2}$. Our model shows that at about this intensity, tunnel ionization becomes important (Figure 4.16). Thus, it appears that the avalanche can be held off by non-polar scattering until intensities in the pulse high enough for tunnel ionization to become dominant are reached. This critical tunnel ionization intensity $\left(\sim 10^{13} \mathrm{~W} / \mathrm{cm}^{2}\right)$ found by Bloembergen is also in good agreement with the intensity where plasma begins to form, as shown by the initial reflectivity rise in Figures $4.2(\mathrm{~g})-(\mathrm{h})$. Furthermore, avalanche hold-off is a general mechanism that is not sensitive to lattice defects and amorphous structure [158], or to band structure details. Seitz [162] emphasized that the collision time for electrons with energies between 3 and $10 \mathrm{eV}$ is short $\left(-10^{-15} \mathrm{~s}\right)$ in any material, citing the same non-polar phonon interaction Braunlich mentions. This collision time implies a mean free path of about one interatomic distance for electrons with energies between 3 and $15 \mathrm{eV}$ [14]. Since the collision distance cannot be any shorter than an interatomic distance, electron-phonon resistivity is expected to saturate and decrease at higher temperatures where the electron-phonon collision crosssection decreases. A similar resistivity saturation effect should occur beyond melting in an ionized solid when the electron-ion collision distance is about one interatomic spacing. This was seen by Milchberg [3] at an intensity of $10^{14} \mathrm{~W} / \mathrm{cm}^{2}$ and electron temperature of about $40 \mathrm{eV}$. His reflectivity studies of $\mathrm{Al}$ showed a minimum over nearly an order of magnitude which he showed to be equivalent to a resistivity maximum. The $\mathrm{Al}$ and $\mathrm{Au}$ data shown in Figures 4.2 (a)-(b) show a similar minimum at about $10^{14} \mathrm{~W} / \mathrm{cm}^{2}$.

Returning to our model results, Figures 4.14 (a)-(b) also show the onset of plasma formation with respect to peak laser intensity. The plasma is formed 
earlier in time as the peak laser intensity is increased. The electron density curve is nearly constant throughout most of the laser pulse. In Section 4.4.4, the unchanging character of $n_{e}$ is used to derive a closed form solution for $T_{e}$.

The time dependence of $T_{e}$ can be inferred by comparing the shape of $T_{e}$ to the laser pulseshape in Figures 4.14 (a)-(b). $T_{e}$ has the appearance of an integrated laser pulse. We use this observation in the next section. Figures 4.14 (a)-(b) also show that $T_{e}$ is virtually constant at the laser pulse's tail end. We can conclude from this observation that collisional electron cooling is insignificant in initial ionization of solids by ultra-short pulse lasers. As mentioned earlier, cooling due to plasma expansion and heat conduction into the cold bulk material are neglected, because they occur on a longer timescale than the initial plasma formation.

\subsubsection{Closed Form Approximations of $T_{e}$ and $n_{e}$}

The constant character of $n_{e}$ during most of the laser pulse is exploited to derive a formula for the time-dependent temperature. The cooling term in Eq. (4.18) is neglected, because $T_{e}$ is constant after initial heating. Substituting Eqs. (4.17) and (4.22) into Eq. (4.18) leads to

$$
\frac{d\left(\frac{3}{2} k T_{e}\right)}{d t}=\frac{16}{3} \sqrt{\frac{\pi}{3}}\left(\frac{2 \pi e^{2}}{h c}\right)^{3} \lambda^{2} \frac{N_{0}}{n_{m e}}\left(\frac{I_{H}}{k T_{e}}\right)^{3 / 2} I_{\text {peak }} \operatorname{sech}^{2}\left(1.76\left(t-t_{\text {peak }}\right) / \tau\right)
$$

where $n_{e}$ is assumed to be constant over the significant part of the laser pulse.

As previously mentioned, the inverse Bremsstrahlung rate temperature singularity is integrable and can be removed from Eq. (4.24) by multiplying through by $T_{e}^{3 / 2}$, and then integrating $T_{e}^{3 / 2} d T_{e} / d t$ to give $(2 / 5) d T_{e}^{5 / 2} / d t$ on the left hand side. The closed form solution of Eq. (4.24) for the initial condition, $T_{e}(0)=0 \mathrm{eV}$, is 


$$
k T_{e}=I_{H}\left\{\frac{5}{3} \frac{16}{3} \sqrt{\frac{\pi}{3}}\left(\frac{2 \pi e^{2}}{h c}\right)^{3} \frac{N_{0}}{n_{\text {me }}} \frac{I_{\text {pak }} \lambda^{2} \tau}{1.76}\left[\tanh \left(\frac{1.76\left(t-t_{\text {park }}\right)}{\tau}\right)+\tanh \left(\frac{1.76 t_{\text {peat }}}{\tau}\right)\right]\right\}^{2 / 5}
$$

In Figures 4.19 (a)-(b), Eq. (4.25) is compared to numerical solutions for $T_{e}$ for two cases illustrated in Figures 4.14 (a)-(b), respectively. At $10^{14} \mathrm{~W} / \mathrm{cm}^{2}$, Eq. (4.25) agrees qualitatively with the numerical solution, and is an upper limit for $T_{e}$ because cooling is neglected. Agreement between the analytic formula and the numerical solution improves dramatically at $10^{16} \mathrm{~W} / \mathrm{cm}^{2}$. Agreement improves with increasing intensity, because the assumption of constant electron density inside the laser pulse is more valid at higher intensities. Therefore Eq. (4.25) is a high-intensity approximation for $T_{e}$ and also an upper limit of $T_{e}$ for all intensities.

The long time limit of Eq. (4.25) is approximately equal to the electron's maximum attainable temperature by laser heating through inverse Bremsstrahlung. The limiting form of Eq. (4.25) is a formula that relates electron temperature to peak laser intensity. For the case where $T_{e}$ starts out with a zero temperature at $t$ equal to minus infinity, the electrons reach a maximum final temperature of

$$
k T_{e}(\infty)=I_{H}\left[2 \frac{5}{3} \frac{16}{3} \sqrt{\frac{\pi}{3}}\left(\frac{2 \pi e^{2}}{h c}\right)^{3} \frac{N_{0}}{n_{a u}}\left(\frac{I_{H}}{k T_{e}}\right)^{3 / 2} \frac{I_{\text {pauk }} \lambda^{2} \tau}{1.76}\right]^{2 / 5}
$$

Eq. (4.26) is Eq. (4.25) with each hyperbolic tangent replaced by 1, its value at $t$ equals infinity. Note the electron temperature scaling with peak intensity, $T_{e} \sim\left(I_{\text {paxk }}\right)^{2 / 5}$, agrees with the work of Murnane [6] and Caruso [163]. A comparison of final temperature, determined by Eq. (4.26), to its numerical counterpart in Figure 4.19 shows this formula is reliable in the high-intensity regime $\left(>10^{14} \mathrm{~W} / \mathrm{cm}^{2}\right)$. 

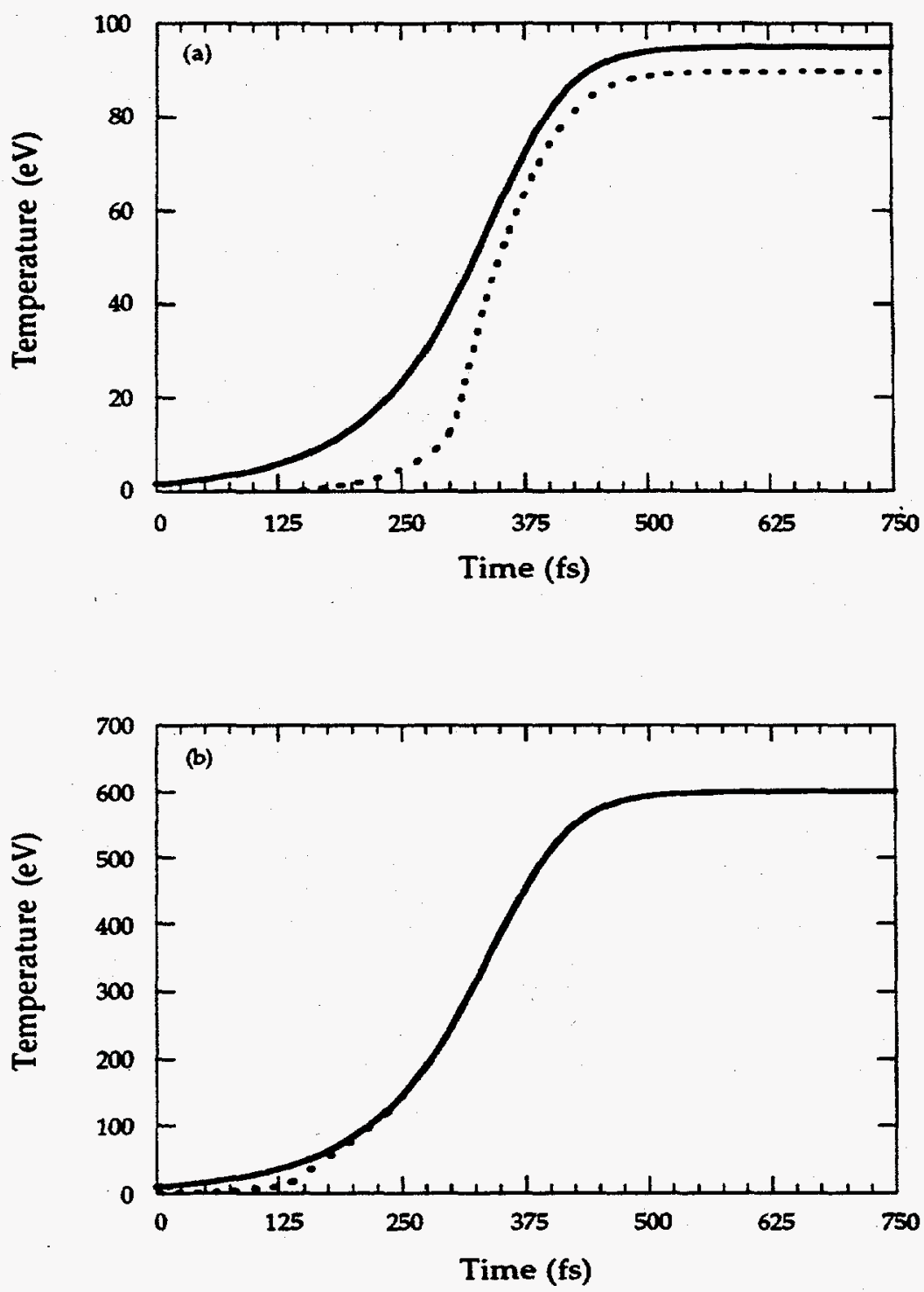

Figure 4.19: (a) Comparison of Eq. (4.25), the analytic form for the $T_{e}$ (solid), and the numerical solution given in Figure 4.14 (a) for $T_{\epsilon}$ (dash), which results from energy deposition of a $10^{14} \mathrm{~W} / \mathrm{cm}^{2}$ laser pulse, shows a qualitative agreement between the two solutions. This comparison and the comparison given in Figure 4.19 (b) show that Eq. (4.25) is a high-intensity approximation for the electron temperature that breaks down near $10^{14} \mathrm{~W} / \mathrm{cm}^{2}$. (b) The same as in (a), but the laser pulse's peak intensity is increased to $10^{16} \mathrm{~W} / \mathrm{cm}^{2}$. Agreement between the two solutions improves with increasing laser intensity, because the assumption that electron density is constant ooer most of the laser pulse width becomes more oalid as peak laser intensity is increased. Behavior of $n_{e}$ with respect to peak laser intensity is exhibited in Figures 4.14 (a)-(b). 


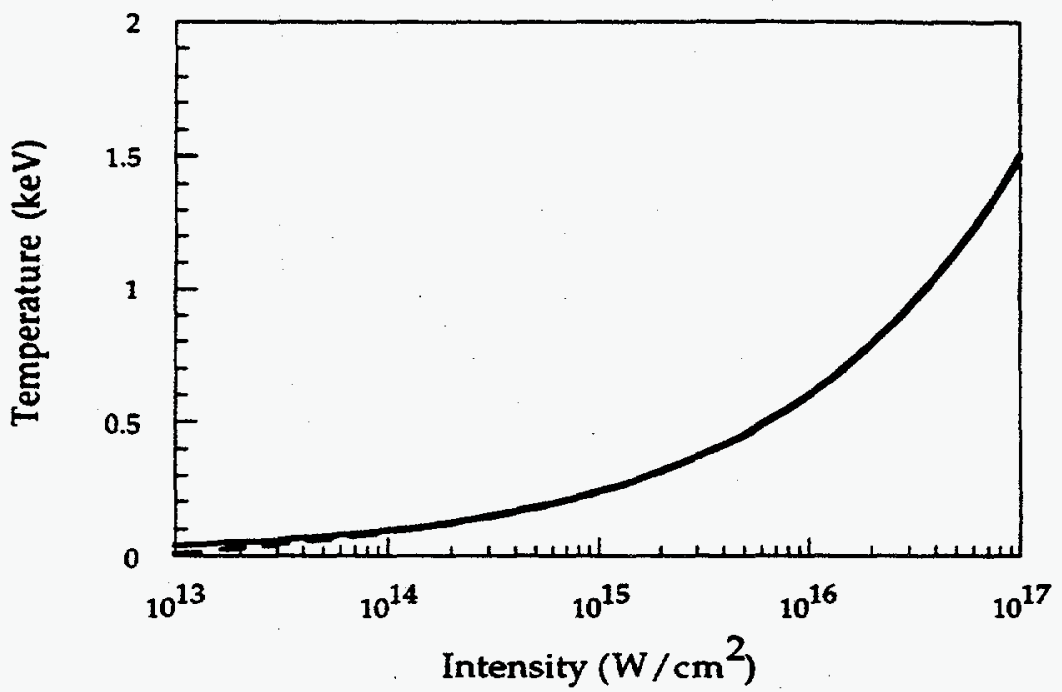

Figure 4.20: The final electron temperature determined from Eq. (4.26) (solid) is compared to its numerical counterpart (dash). This comparison shows Eq. (4.26) is reliable in the high-intensity regime.

Eq. (4.16) can be integrated analytically in a small time interval because its coefficients are approximately time-independent. Coefficients in an interval are determined from laser intensity and electron temperature taken at the interval midpoint. Electron temperature at the midpoint is determined from Eq. (4.25). With omission of recombination, electron density in an interval is

$$
n_{e}=N_{0} \frac{\left(1-n_{e}(0) / N_{0}\right)^{-1}-\left(1+\alpha n_{e}(0) / \omega_{t}\right)^{-1} \exp \left(-\left(\alpha N_{0}+\omega_{t}\right) \delta t\right)}{\left(1-n_{e}(0) / N_{0}\right)^{-1}+\left(\alpha N_{0} / \omega_{t}\right)\left(1+\alpha n_{e}(0) / \omega_{t}\right)^{-1} \exp \left(-\left(\alpha N_{0}+\omega_{t}\right) \delta t\right)}
$$

where $n_{e}(0)$ is the electron density at the beginning of the time interval $\delta t$. In Figures 4.21 (a)-(b), Eq. (4.27) is compared to numerical solutions for electron density given in Figures 4.14 (a)-(b), respectively. The difference between Eq. (4.27) and the numerical solution decreases with increasing intensity and is almost indiscernible at $10^{16} \mathrm{~W} / \mathrm{cm}^{2}$. 


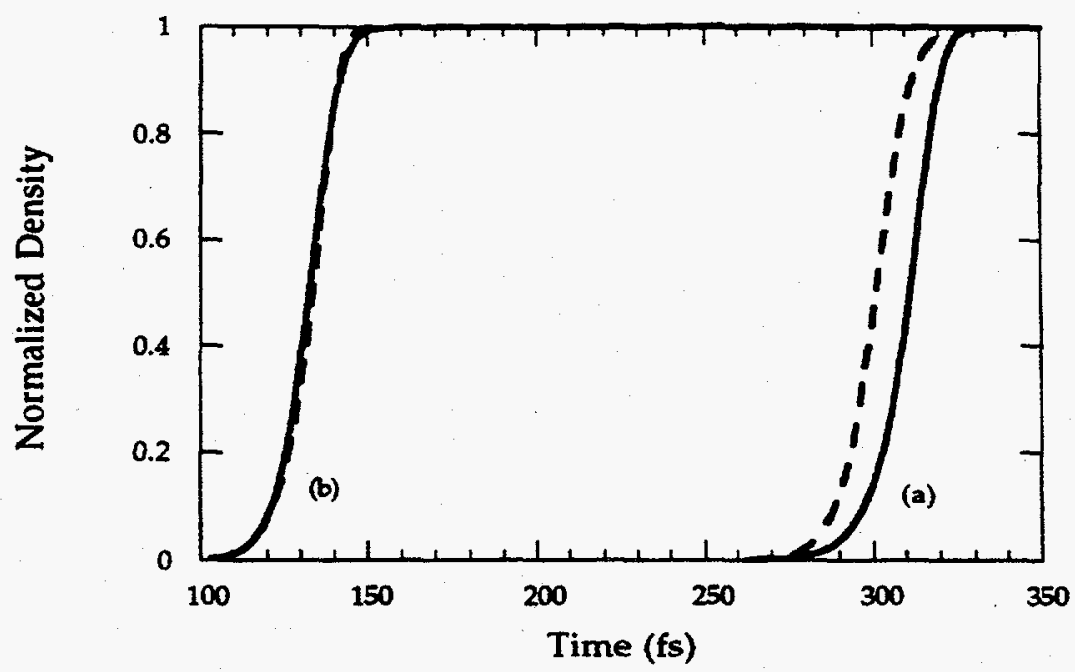

Figure 4.21: (a) Comparison of Eq. (4.27), the analytic form for the $n_{e}$ (solid), and the numerical solution given in Figure 4.14 (a) for $n_{e}$ (dash) shows Eq. (4.27) to be an accurate representation of the time-dependent density produced by a $10^{14} \mathrm{~W} / \mathrm{cm}^{2}$ laser pulse. (b) The same as in (a), but the laser pulse's peak intensity is $10^{16} \mathrm{~W} / \mathrm{cm}^{2}$.

Electron density reached at the pulse's end can also be determined in closed form. Since impact ionization contributes negligibly to electron density, $\alpha\left(N_{0}-n_{e}\right) n_{e}$ can be omitted from Eq. (4.16) in the final electron density derivation. The electron production equation is simply $d n_{e} / d t=\omega_{t}\left(N_{0}-n_{e}\right)$. At $t$ equal to infinity, electron density determined from this approximation is [110].

$$
n_{e}(\infty)=N_{0}[1-\exp (-\phi)]
$$

where the exponential argument is

$$
\phi=4 \omega_{a u} \frac{2 \tau}{1.76} \frac{E_{p}^{5 / 2}}{F_{\text {peak }}} K_{1}\left(\frac{2 E_{p}^{3 / 2}}{3 F_{\text {peak }}}\right)
$$

$K_{1}$ in Eq. (4.28) is the Modified Bessel Function of order one [164]. Eq. (4.28) is compared to numerical results obtained from Eq. (4.16) in Figure 4.22. 


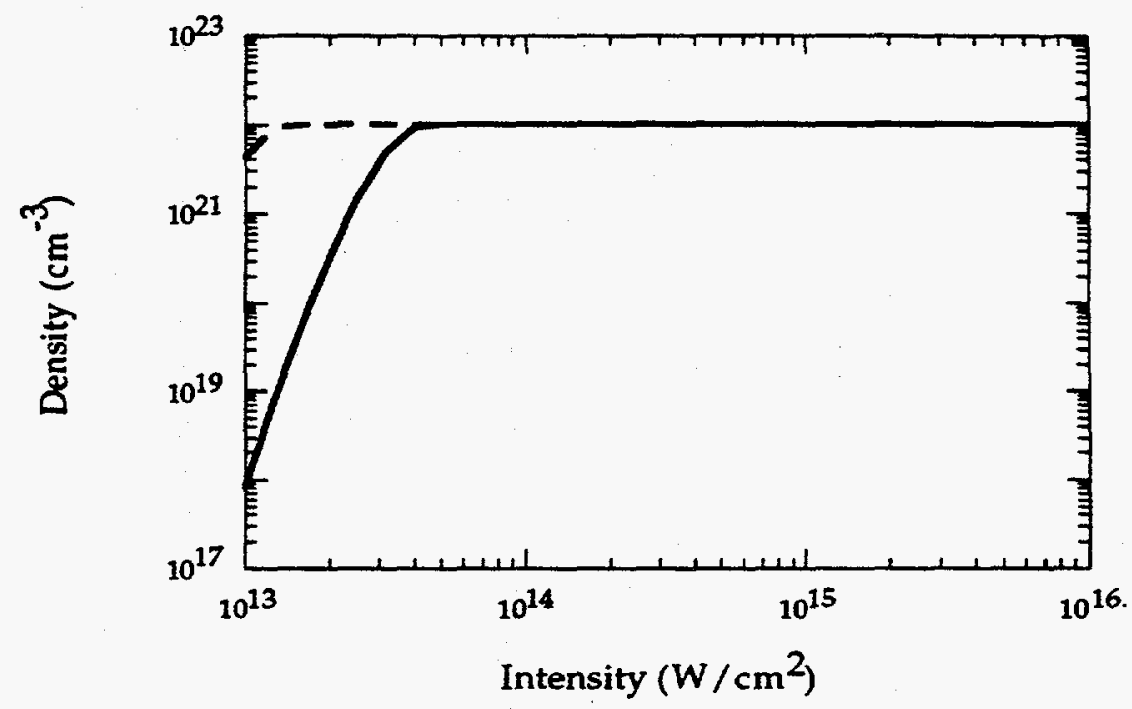

Figure 4.22: Final electron density determined from Eq. (4.28) (solid) is compared to the numerical result (dash). This comparison shows that Eq. (4.28) is a high-intensity approximation for electron density.

\subsubsection{The Nonequilibrium Nature of $n_{e}$}

In thermal equilibrium, plasma ionization is completely determined by temperature through the Saha equation. If the plasma temperature is changed slowly enough that the plasma can be considered to be in a LTE state, then the new degree of ionization can be determined from the Saha equation at the new temperature. The equilibration time is the time it takes for the plasma ion distribution to adjust to the new temperature, and it is related to $\alpha$ and $\rho$. A necessary condition for the plasma to be in a LTE state is the equilibration time must be smaller than the time taken to change the temperature. However, we show that for ultra-short pulse plasmas this is not a sufficient condition for LTE.

Figures 4.14 (a)-(b) show electron temperature increases almost in parallel with the laser pulse's leading edge. Thus, a laser heats a plasma at a rate on the order of the inverse laser pulsewidth $1 / \tau$. This conclusion can also be drawn from Eq. (4.24), since $\tau$ is the only time scale in the equation. 
The linear theory of perturbations on nonlinear differential equations can be applied to Eq. (4.16) to determine equilibration time for an isolated plasma. For a plasma in the absence of a field, photo-ionization in Eq. (4.16) is zero. The stationary solution $\underline{n}_{\epsilon}$ of Eq. (4.16) for an isolated plasma, also known as the equilibrium electron density, is determined by setting the right hand side to zero, i.e., $\alpha\left(N_{0}-\underline{n}_{e}\right) \underline{n}_{e}-\rho \underline{n}_{e}^{3}=0$. This is the Saha equation. It specifies the degree of ionization $\underline{n}_{e} / N_{0}$ in terms of $\alpha / \rho$. Note that for Saha equilibrium, when atoms are multiply ionized, we will have a system of equations, one for each ion state. We want to know the rate at which a nonstationary state, $n_{e}$, relaxes to the stationary state, $\underline{n}_{e}$, when $n_{e}$ is close to $\underline{n}_{\ell}$. The two states are related as $n_{e}=\underline{n}_{\varepsilon}+\Delta n_{e}$, where $\Delta n_{e}$ represents the time-dependent perturbation from equilibrium. Substituting $n_{e}$ into Eq. (4.16) and retaining terms to first order in $\Delta n_{e}$ yields the equation governing the relaxation of $\Delta n_{e}$

$$
\frac{d \Delta n_{e}}{d t}=-\left(\alpha \underline{n}_{e}+2 \rho \underline{n}_{e}^{2}\right) \Delta n_{e}
$$

According to Eq. (4.30), the old state relaxes to the new in the time of $\left(\alpha \underline{n}_{e}+2 \rho \underline{n}_{e}^{2}\right)^{-1}$, which we call the equilibration time $\tau_{e q}$. The Saha equation can be solved explicitly for $\underline{n}_{e}$ to give $(1 / 2)\left(-\alpha / \rho+\left(\alpha^{2} / \rho^{2}+4 N_{0} \alpha / \rho\right)^{1 / 2}\right)$. Substituting this result into $\tau_{e \text {, }}$ gives equilibration time as a function of the rate coefficients $\alpha$ and $\rho$,

$$
\tau_{e q}^{-1}=\sqrt{\alpha^{2}+4 \alpha \rho N_{0}}\left(-\alpha+\sqrt{\alpha^{2}+4 \alpha \rho N_{0}}\right)(2 p)^{-1}
$$

Figure 4.23 is a plot of Eq. (4.31) as a function of $T_{c}$, and shows the equilibration time is smaller than 10 fs for electron temperatures above $\sim 3 \mathrm{eV}$. Therefore, an isolated plasma whose temperature is greater than $\sim 3 \mathrm{eV}$ can be maintained in a LTE state even when the temperature is increased at a rate as fast as $1 \mathrm{eV}$ every few femtoseconds. 


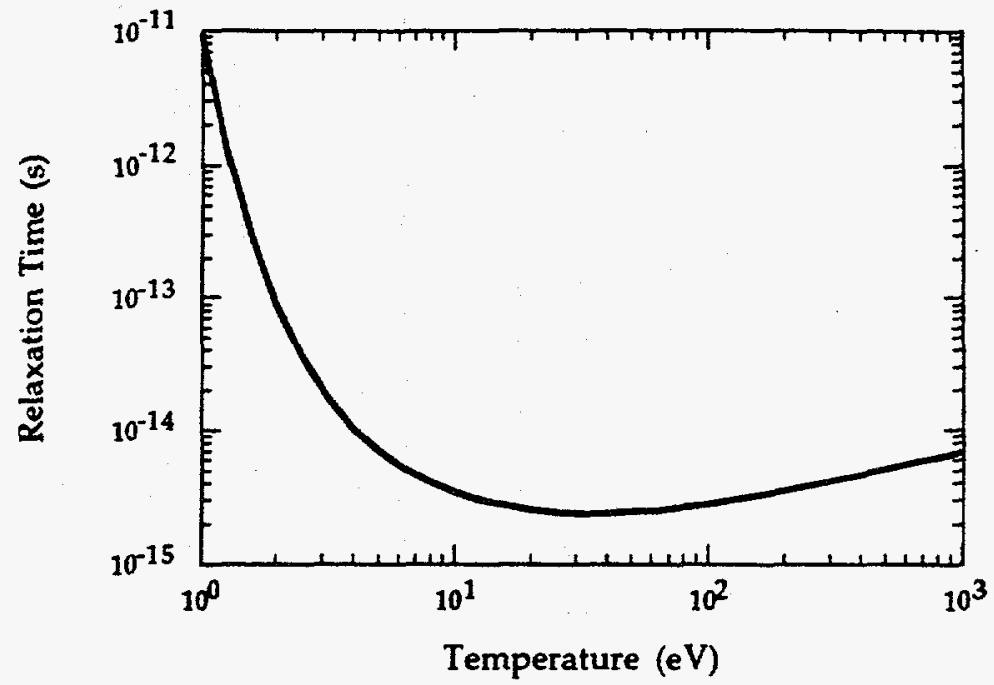

Figure 4.23: Plot of Eq. (4.31), the relaxation time, as a function of the electron temperature.

It is a mistake, however, to believe that high-intensity $\left(>10^{14} \mathrm{~W} / \mathrm{cm}^{2}\right)$ ultra-short pulse ( 100fs) lasers produce plasmas in LTE only because the equilibration rate $1 / \tau_{e q}>10^{13} \mathrm{~s}^{-1}$ is faster than the heating rate $1 / \tau \sim 10^{13} \mathrm{~s}^{-1}$ (the inverse pulsewidth). This is a misconception, because the photo-ionization rate $\omega_{t}$ is much larger than the equilibration rate. Plasma does not have time to adjust thermally to ionization induced by the laser. In order for a state of local thermodynamic equilibrium to be maintained in the plasma, the collisional equilibration rate must be the largest rate during the ionization process. A comparison of $1 / \tau_{e q}$ and $\omega_{t}$ is given in Figure 4.24 in which Eq. (4.26) is used to determine $T_{e}$ for Eq. (4.31). This figure shows the photo-ionization rate is at least an order of magnitude larger the equilibration rate for laser intensities greater than $3 \times 10^{13} \mathrm{~W} / \mathrm{cm}^{2}$. Therefore, the assumption that LTE is maintained in an ionizing plasma breaks down during formation at higher intensities. This is consistent with our observation in Section 4.4.3 that the plasma formation at these 


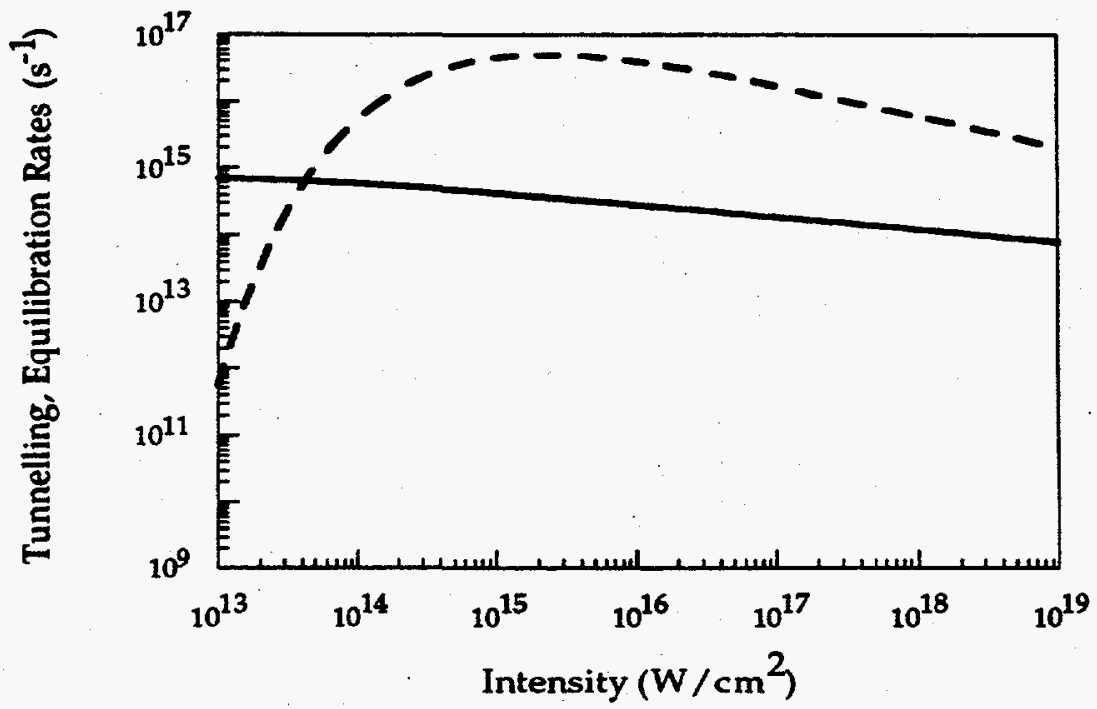

Figure 4.24: A comparison of $1 / t_{e q}$ (solid) and the tunneling ionization rate (dash).

intensities is laser-driven not temperature-driven, i.e., the contribution to $n_{e}$ by impact ionization is negligible.

\subsubsection{The Self-Induced Reflection of a Laser Pulse}

Self-induced plasma reflectivtiy was calculated by the method of Born and Wolf [165] for determining reflection coefficients of multi-layered media. Born and Wolf's matrix treatment is entirely equivilant to solving the Helmholtz differential equations. In this treatment, the medium is divided into a large number of thin slabs of constant depth. The slab thickness is the only adjustable parameter in our calculations. It is fixed by the smallest skin depth that can be obtained by our laser which occurs at the highest intensity $\left(>10^{16} \mathrm{~W} / \mathrm{cm}^{2}\right)$. In a small time interval, the evolving plasma density, Eq. (4.27), and temperature, Eq. (4.25), in each slab are treated as constants. These are then substituted into Drude's model [166] for the plasma dielectric constant to determine the slab's optical response. The total dielectric constant of a slab 


$$
\varepsilon=\varepsilon_{0}-\frac{n_{e} / n_{c r i t}}{\left(1+i c k_{I B} / \omega_{L}\right)}
$$

is the sum of the unionized atomic dielectric constant, $\varepsilon_{0}-1$, and the breakdown plasma dielectric constant, $1-\left(n_{e} / n_{c r i t}\right) /\left(1+i c k_{I B} / \omega_{L}\right)$. Here, $n_{c r i t}=m_{e} \omega^{2} / 4 \pi e^{2}$ is the critical electron density above which the incident electromagnetic wave is evanescent and $c k_{I B}=2 \pi \gamma$, where $\gamma$ is the inverse Bremsstrahlung collision frequency used in Eq. (4.33). This approach has been used previously for semiconductors and metals $[3,13,114,167]$. The dielectric constant of Eq. (4.32) determines Born and Wolf's $2 \times 2$ characteristic matrix for a slab. Total medium reflectivity is determined from the matrix product of all the slabs' characteristic matrices.

It is more convenient to express results in terms of plasma conductivity than the plasma dielectric constant. The dielectric constant is related to the conductivity by $\varepsilon-\varepsilon_{0}=i(4 \pi / \omega) \sigma$, where $\varepsilon$ is the complex dielectric constant, $\sigma$ is the conductivity, and $\omega$ is the laser frequency. Using this relation with the Drude model, the real plasma conductivity used for ohmic losses $\sim \sigma_{r} E^{2}$ is,

$$
\sigma_{r}=\frac{\omega_{p}^{2} \gamma}{4 \pi\left(\gamma^{2}+\omega^{2}\right)}
$$

This conductivity has been used successfully in previous detailed analyses of the conductivity temperature behavior for dense plasmas [147]. From the preceding model, reflectivity is calculated as a function of intensity and compared with data in Figure 4.25. The single free parameter in the fit is a variable skin depth that is fixed as discussed above. Note that diffusely reflected light is not accounted for. This is discussed below. 


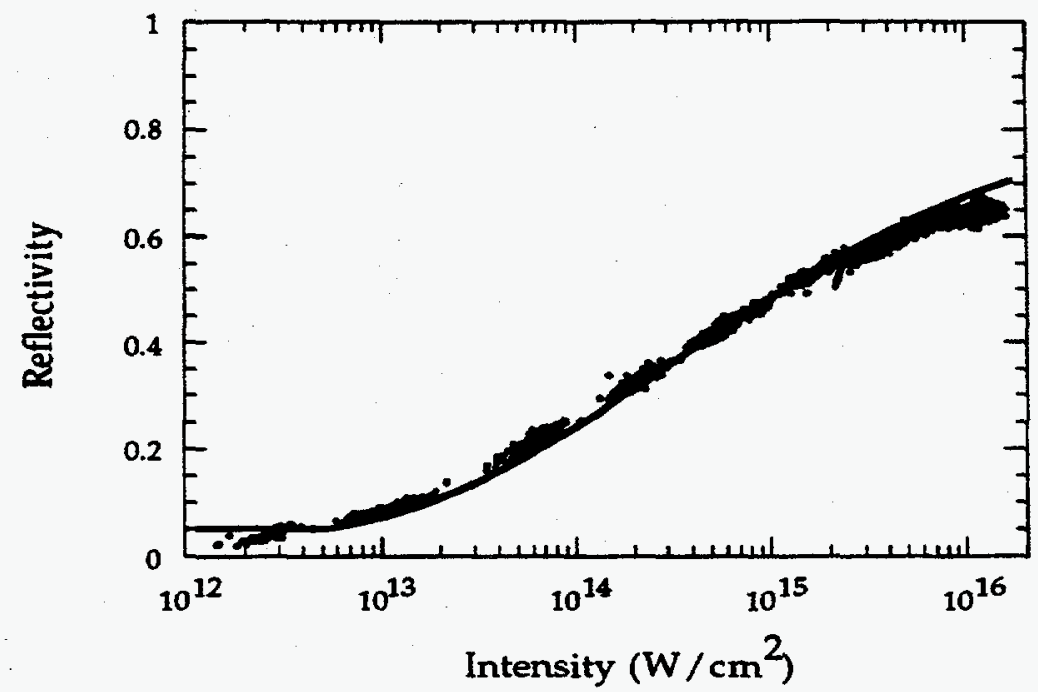

Figure 4.25: Calculated reflectioity versus intensity for the real Drude conductivity model is compared to reflectioity data in quartz $\left(\mathrm{SiO}_{2}\right)$.

Finally, to examine the restrictive nature of our assumption that the plasma can be at most singly ionized, we relax this restriction and allow for multiple ionization. Reflectivity curves determined from our single ionization (solid line) and multiple ionization (dashed line) models are compared to experimental data in Figure 4.26. We find multiple ionization improves the qualitative (curve shape) agreement between theory and experiment at the highest and lowest measured intensities. The $\sim 5-10 \%$ higher absolute reflectivity obtained by the multiple ionization model is discussed below. Note that contributions to the electron density from multiple ionization beyond a supercritical density has a small $(<10 \%)$ effect on the time-integrated self-reflectivity when compared to the single ionization model. This justifies our single ionization model as an adequate quantitative estimate of reflectivity and an excellent predictor of qualitative behavior. The single ionization model also avoids the complication of choosing multiple ionization energies for solid state materials that are largely unknown. Most importantly, closed form solutions in 


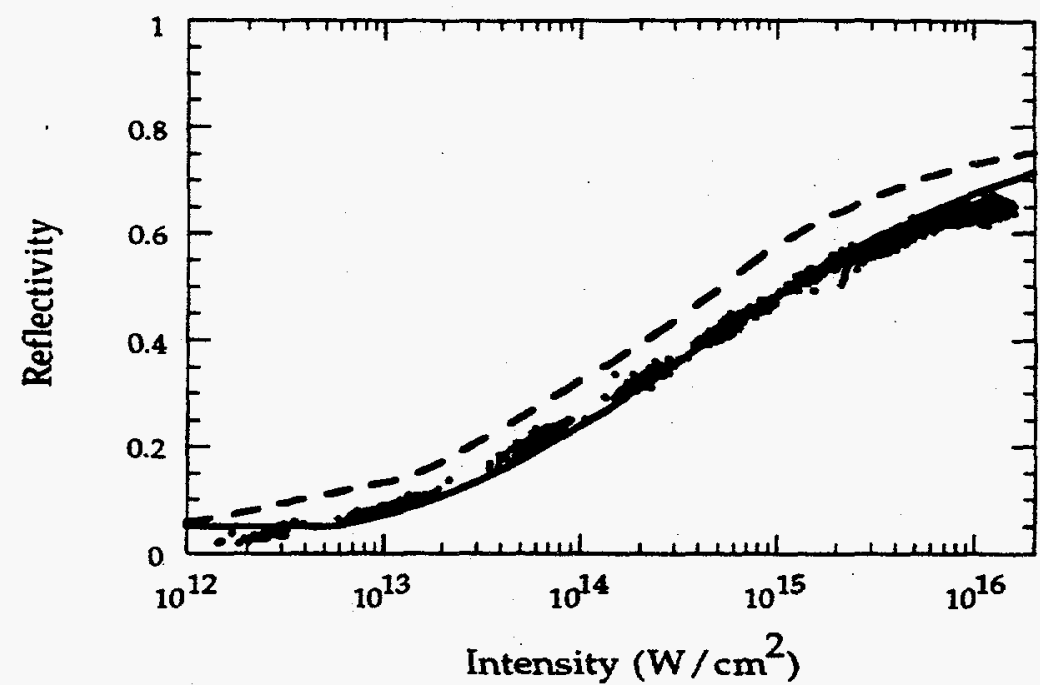

Figure 4.26: Measured reflectivity as a function of incident pulse intensity using a quartz target is compared to the single ionization (solid) and the multiple ionization (dash) non-LTE model.

the single ionization model provide insight into the scaling physics involved in this interaction.

The multiple ionization curve gives an excellent qualitative fit to the data, but overestimates reflectivity by about $5 \%$ at the lower intensities and $10 \%$ at higher intensities. The difference may be accounted for by the nonspecular reflected light. Our experiment measures only specularly reflected light, while the theory predicts the total reflected light (specular + diffuse). This laboratory [168] measured a nonspecular reflectivity which monotonically increased from about $3 \%$ to $10 \%$ for intensities from $10^{13} \mathrm{~W} / \mathrm{cm}^{2}$ to $10^{16} \mathrm{~W} / \mathrm{cm}^{2}$ using a float glass target and low-prepulse, $400 \mathrm{~nm}, \sim 100 \mathrm{fs}$ laser pulses. This figure is in reasonable agreement with the discrepancy between the multiple ionization model and the data. The single ionization model will still give reflectivity estimates within $10 \%$ of the data and will underestimate it as expected because of a lower predicted electron density. 


\section{CHAPTER 5:}

\section{SPACE-AND TIME-RESOLVED REFLECTIVITY MEASUREMENTS}

\subsection{INTRODUCTION}

Pump-probe reflectivity measurements spatially and temporally-resolved plasma reflectivity and transmission with a nonintrusive (low-intensity) probe pulse. Plasma was created from an $\mathrm{SiO}_{2}$ (fused quartz) target by the intense $\left(\sim 10^{16} \mathrm{~W} / \mathrm{cm}^{2}\right)$, ultra-short (130fs) laser pump pulse, described in Chapter 2. A nonintrusive fraction of the pump beam was used to probe the plasma. Temporal resolution of $130 \mathrm{fs}$ (probe pulsewidth-limited) was attained by measuring reflectivity as a function of the time delay between the arrival of the pump and the probe pulse. Spatial resolution of $-3 \mu \mathrm{m}$ was achieved by aperturing magnified images of the reflected and transmitted probe beam at the target interaction region.

\subsection{EXPERIMENTAL SETUP}

\subsubsection{Pump-Probe Design}

A schematic experimental pump-probe setup is shown in Figure 5.1. The input beam was the $\sim 3.5 \mathrm{~mJ}, 614 \mathrm{~nm}, \sim 130 \mathrm{fs}$ laser source described in Chapter 2. This input beam was split into a low energy probe beam and a high energy pump beam by a $99 \%$ beamsplitter. To normalize the signal to laser fluctuations, incident pump energy was measured using diode D1 by sampling the beam with a pellicle beamsplitter. The pump beam was directed to an adjustable delay line and focused on the target at normal incidence by a $10 \mathrm{~cm}$ achromatic lens. The probe beam was focused by a $50 \mathrm{~cm}$ singlet lens located outside the chamber with 
an angle of incidence to the target normal of $30 \pm 2^{\circ}$. Specularly reflected and transmitted probe beams were collected by lenses and directed through magnifying-imaging systems (magnification $\sim 360 \mathrm{X}$ ) located outside the chamber. Magnification gave an excellent image of the plasma region $(-15 \mu \mathrm{m})$ magnified to a practical size, $\sim 5 \mathrm{~mm}$, for spatial aperturing in the image plane. The magnified plasma region image, from both the reflected and transmitted sides, was selectively apertured by an iris placed in the image plane just before the diode signal detector. The relative scattered light signal was detected using a diode (D2) placed $\sim 20^{\circ}$ below the reflected probe beam collection lens. The reflected probe diode (D3) could also be replaced by a Pullnix camera (model TM $745 \mathrm{E})$, with its $C C D$ array in the image plane, to view the overlap of the focused pump and probe spots. Real-time video images of the overlap were useful in identifying target misalignment relative to the raster scan plane. When the target was not perfectly aligned with the raster scan plane we observed the focused pump spot walk across the probe spot on the video monitor. The target wheel was mounted on a $\theta, \phi$ micrometer stage, which was adjusted with until no relative walkoff of the pump and probe spots was seen over the entire raster scan range of $1.5 " \times 1.5 "$. Rough optical and target alignment was aided by a HeNe laser beam directed to be near coincident with the femtosecond beam. The vacuum chamber was maintained at a base pressure of $\sim 1 \mu$ Torr. Probe beam polarization was changed between $S$ and $P$ by a zero-order $1 / 2$ wave plate and was estimated to be $98 \%$ linear. Pump pulse energy was adjusted by a zeroorder $1 / 2$ wave plate and polarizer. The targets, their preparation, optimum focusing, pulsewidth measurement and the target raster scan system were previously described in Section 4.2. The zero-delay position between the pump and the probe was established within $\pm 15 \mathrm{fs}$ by studying the well-known reflectivity response of a silicon sample [4, 169-171]. 


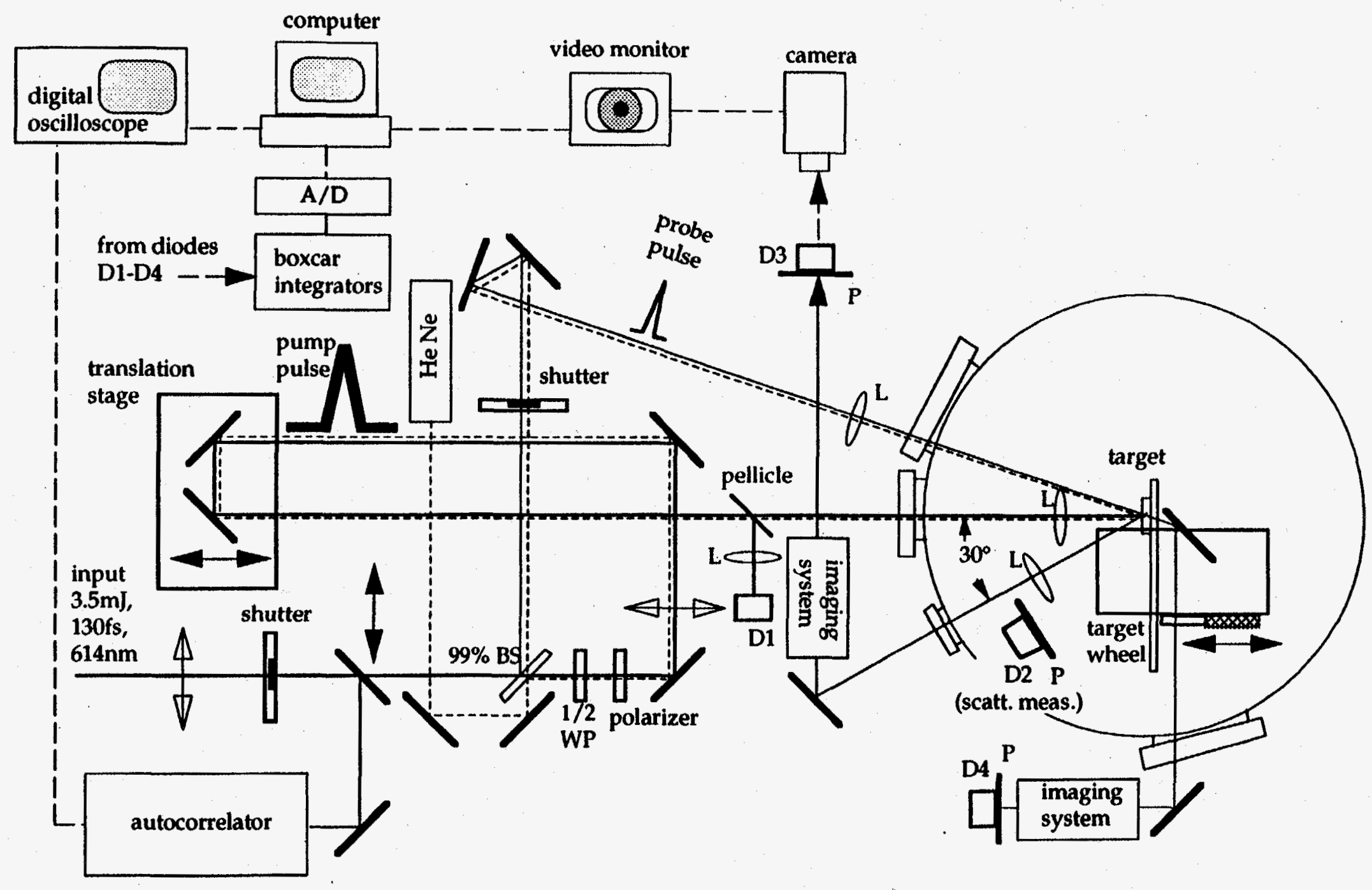

Figure 5.1: Experimental pump-probe setup for space- and time-resolved reflectivity measurements.

(WP=wave plate, $D=$ diode, $P=$ polarizer, $B S=$ beamsplitter $, L=l e n s, A / D=$ analog to digital computer board) 
All light was detected with four diodes (D1-D4, UDT model 10DP) calibrated separately to absolute energy and kept below their saturation signal $(\sim 100 \mathrm{mV})$ by a set of calibrated neutral density filters. The signal-to-noise ratio was improved by placing a Melles-Griot dichroic sheet polarizer (model 03 FPG 003) before the diodes D2-D4. The signals from the diodes were fed into a fast preamplifier (SRS model SR240) and then into a four boxcar integrators (SRS model SR250) operating in sample-and-hold mode. Each sample-and-hold signal was then sampled (100samples/shot) by a National Instruments $A / D$ computer board (model NB-MIO-16XL) in a Macintosh IIfx computer. A/D samples were averaged (100 sample averages) for each shot and the shots were also averaged (10 shot averages). The single-shot measurements were also recorded, but the averaged values were generally found to be more useful in the analysis. Background light and electronic drift was subtracted by running the sampling rate in the boxcar at twice the laser frequency and using every other sample (shutter closed) as a background signal. All signal detection, data sampling and recording, raster and delay line scanning and shutter operation was synchronized by a Labview 2 virtual instrument program interfaced with the Macintosh IIfx computer via GPIB protocol. The virtual instrument program also allowed real-time reflected signal averages to be displayed on the computer, so target misalignment or other systematic experimental errors were quickly detected and corrected by looking for a smooth, unsaturated signal. For example, a small target misalignment caused the reflectivity signal to oscillate as the probe beam walked across the central plasma region. An example of this oscillation is shown in Figure 5.2 to illustrate how easy it was to detect anything less than perfect target alignment. 


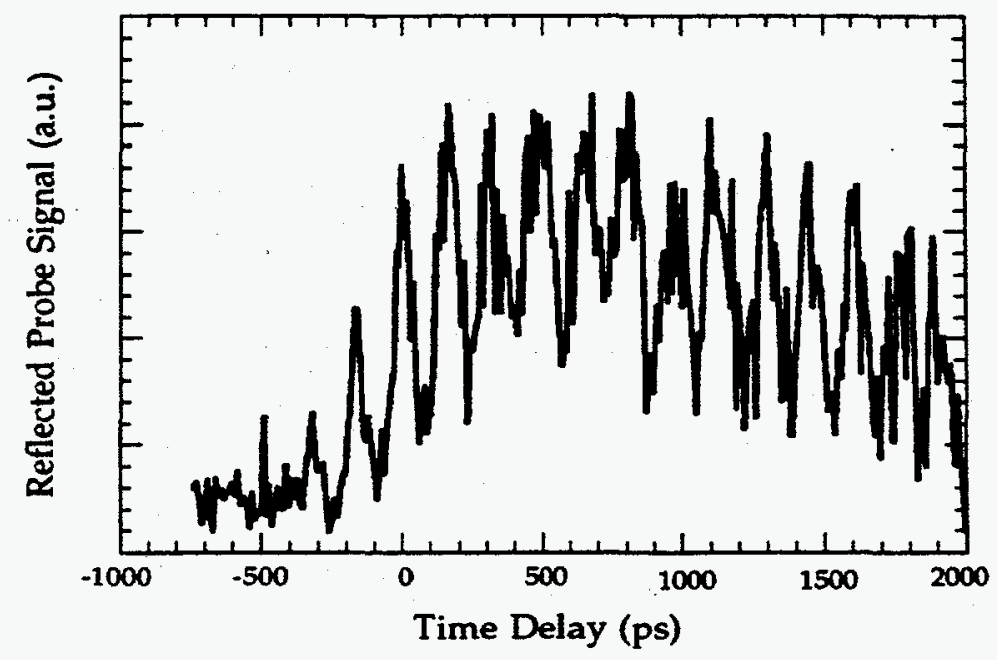

Figure 5.2: An imperfect target alignment displayed an oscillatory reflection signal. .

\subsubsection{Absolute Calibration Measurements}

The unionized target absolute reflectivity was determined by comparing the cold reflectivity value measured for each target with the reflectivity value obtained for negative time delays (probe precedes pump). The cold reflectivity of each target was measured over a large range of angles (Figure 5.3) for comparison to published values to be certain of the accuracy of reflectivity values used for the single angle $\left(30^{\circ}\right)$ measurement in the experiment.

Plasma reflectivity values were determined by comparing reflected plasma signal counts with a linear interpolation of counts obtained from measured low-intensity reflectivity counts of a calibration target. The calibration target consisted of $1 / 3 \mathrm{Al}, 1 / 3 \mathrm{Si}$ and $1 / 3$ uncoated $\mathrm{SiO}_{2}$. A calibration target run is shown in Figure 5.4. Since the low-intensity reflectivity of the calibration target materials was previously measured, the absolute plasma reflectivity could be determined for any set of amplifier or A/D board gains and neutral density filters used on the diodes. 


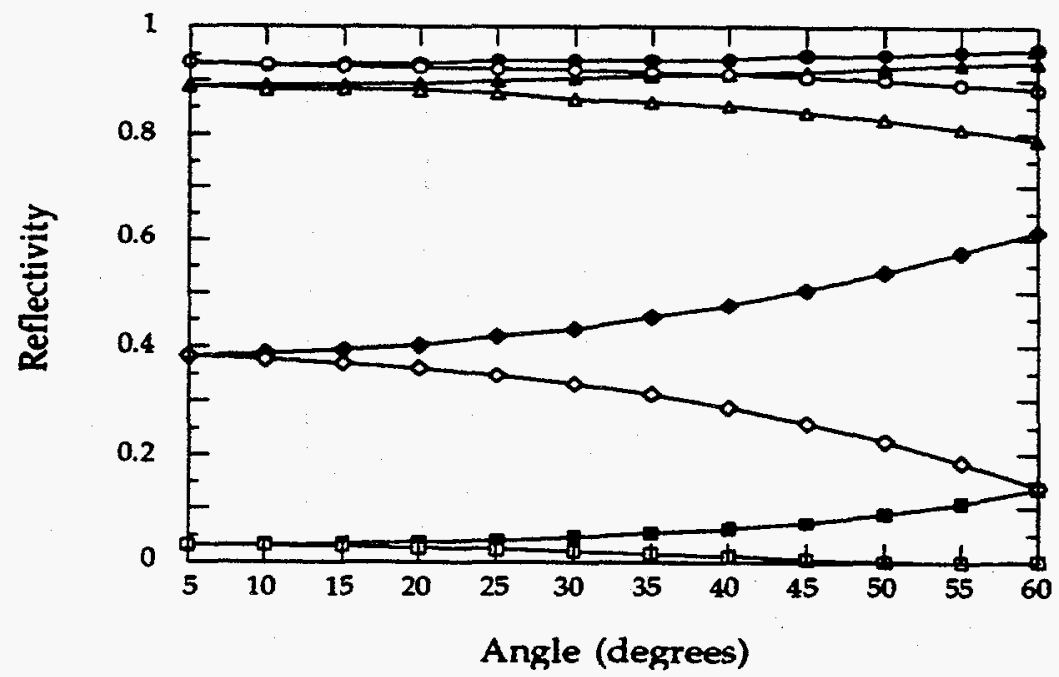

Figure 5.3: Measured low-intensity reflectivity curves for $\mathrm{SiO}_{2}$ (squares), $\mathrm{Si}$ (diamonds), $A l$ (triangles) and $A u$ (circles) for $S$ (solid) and $P$ (open) polarization.

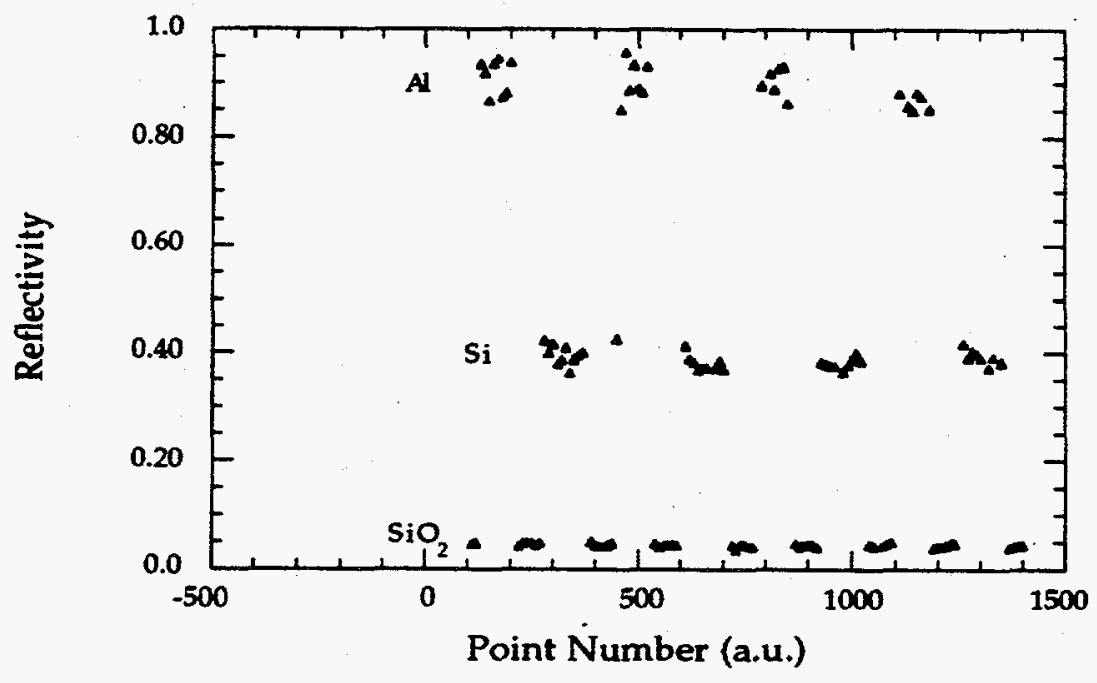

Figure 5.4: A linear interpolation of the low-intensity reflectioity counts from the calibration target is used to determine absolute reflectivity values. 
The absolute energy incident on the target was determined by comparing the diode signal counts with a measured calibration of signal counts versus energy. A linear interpolation allowed determination of the absolute energy as long as the diodes were kept in the linear response regime $(<100 \mathrm{mV})$ by neutral density filters.

\subsubsection{Spatial Imaging System}

Two general geometries are used in pump-probe experiments to avoid spatial averaging over a transverse intensity distribution. First, a small probe spot can be centered on a larger pump spot (Figure 5.5). Second, a large probe spot can be used to illuminate the entire, smaller, pump spot, $z$ and all but the center of the imaged interaction region is apertured out. The latter was chosen because it has several advantages. The most obvious advantage is that the large probe method maximizes the intensity available for the pump by minimizing the spot size, while lowering the probe intensity to keep it nonintrusive. A second advantage is that a large probe geometry is less sensitive to walkoff due to target misalignment. If the pulses have a Gaussian profile, a small walkoff in the small probe geometry will sample a much different plasma temperature and density region. A small walkoff in a large probe geometry, however, will still illuminate the entire plasma and suffer only a minor change in probe intensity. The large probe geometry also allows one to spatially resolve any portion of the plasma region by aperturing a magnified image of the plasma in the image plane. The iris was centered by setting the pump pulse several picoseconds earlier in time than the probe and centering the iris on a central dark region caused by absorption in the hydrodynamic blowoff, as discussed by Downer [170]. This spatially resolved technique was used in this work on both the reflected and transmitted probe beams as shown in Figure 5.5. Results of the spatially resolved 
measurements are presented below. Finally, with the large probe technique, one can determine the physical size of the critical density region. By closing a centered aperture down until the transmitted signal is zero at zero time delay one can obtain an estimate of the size of the critical surface.

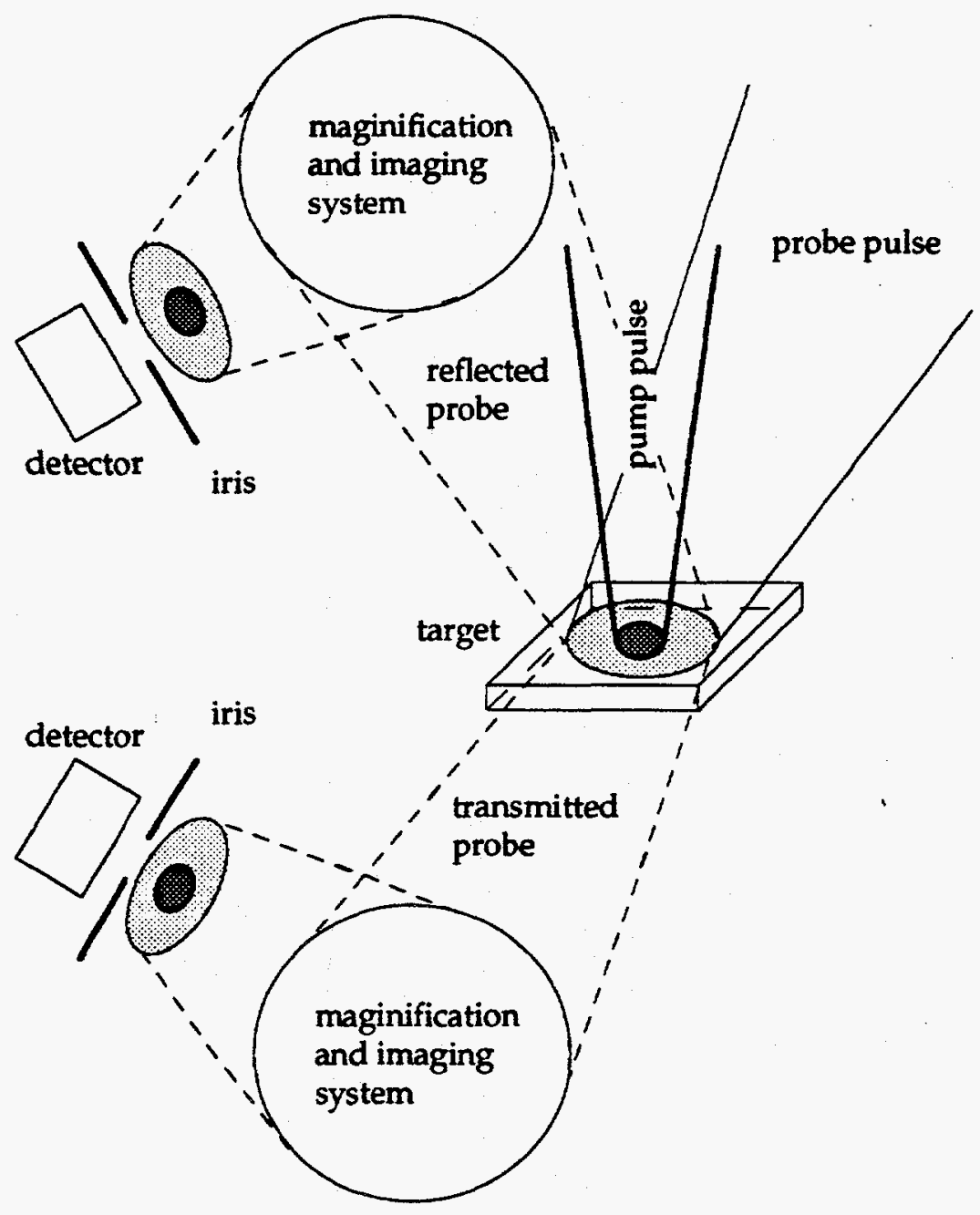

Figure 5.5: The reflected and transmitted signal are spatially resolved over the plasma profile. 


\subsection{EXPERIMENTAL RESULTS AND DISCUSSION}

\subsubsection{Reflectivity, Transmission and Scatter Measurements}

Reflectivity, transmission, and a relative scatter signal for the central plasma region created by an intensity of $5 \times 10^{15} \mathrm{~W} / \mathrm{cm}^{2}$ are shown in Figure 5.6. The central plasma region is approximately a $3 \mu \mathrm{m}$ diameter spot in the center of the $15 \mu \mathrm{m}$ diameter pump focal spot. This figure was measured by minimizing the aperture in the image plane and resolving a $3 \mu \mathrm{m}$ wide bar on a resolution target (Newport RES-1) placed at the target position.

The reflectivity displays a pulsewidth-limited risetime followed by the rapid relaxation to zero, $\sim 4 \%$ below it initial, cold value. Transmission falls in a pulsewidth-limited manner as it is cut off immediately upon critical plasma formation. Scattered light was measured with a single diode placed $20^{\circ}$ below the collection lens for the specularly reflected light and $30^{\circ}$ to the target normal. This single-diode method obviously does not give an absolute measurement of the total scattered light. Instead, it was used as a relative indication of any significant changes in the scattered light level. Complete capture of all scattered light requires use of a large integrating sphere which was not possible in this experimental chamber. The scatter diode was tested at a number of positions around the target with no significant difference in the relative signal behavior. The relative signal only indicates the percentage fluctuation of the scatter signal. This signal rises slowly over the entire scan with a few significant intermediate fluctuations. The average change, however, over the entire scan was $<5 \%$. 


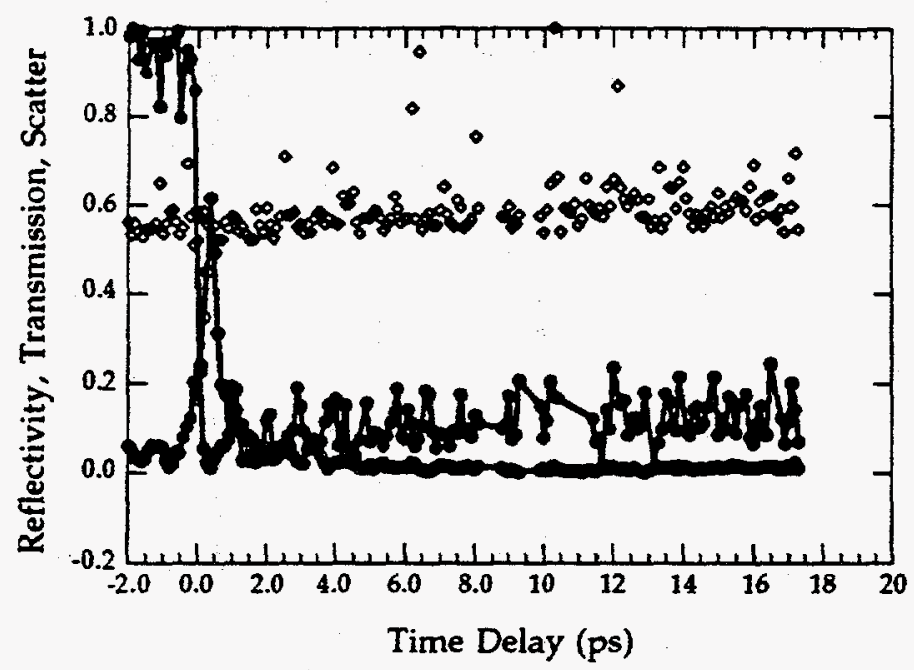

Figure 5.6: Reflectivity (open circle), transmission (closed circle) and relative scatter (diamond) from an $\mathrm{SiO}_{2}$ target irradiated with an S-polarized, $5 \times 10^{15} \mathrm{~W} / \mathrm{cm}^{2}$ pump pulse. Irises aperture in the transmitted and reflected image planes, so that only the central $\sim 3 \mu \mathrm{m}$ of the plasma is detected.

\subsubsection{Spatially-Resolved Reflectivity}

Spatially resolving the plasma demonstrates that a spatially-integrated experiment measures the convolution of a number of different plasma states. Reflectivity measurements at three different imaging aperture sizes, but constant pump intensity, for $\mathrm{SiO}_{2}$ and S-polarized light are shown in Figure 5.7. The highest ( $80 \%$ peak), sharpest peak shown has the smallest imaged region $(\sim 3 \mu \mathrm{m}$ diameter) at the center of the plasma. As more of the plasma is imaged (as the aperture is opened) peak reflectivity decreases and relaxation time increases. The lowest reflectivity curve shown ( $42 \%$ peak) is the case of no aperture, i.e., a spatially-integrated measurement. We now investigate how the focal intensity distribution explains this measured distribution of peak plasma reflectivities. 


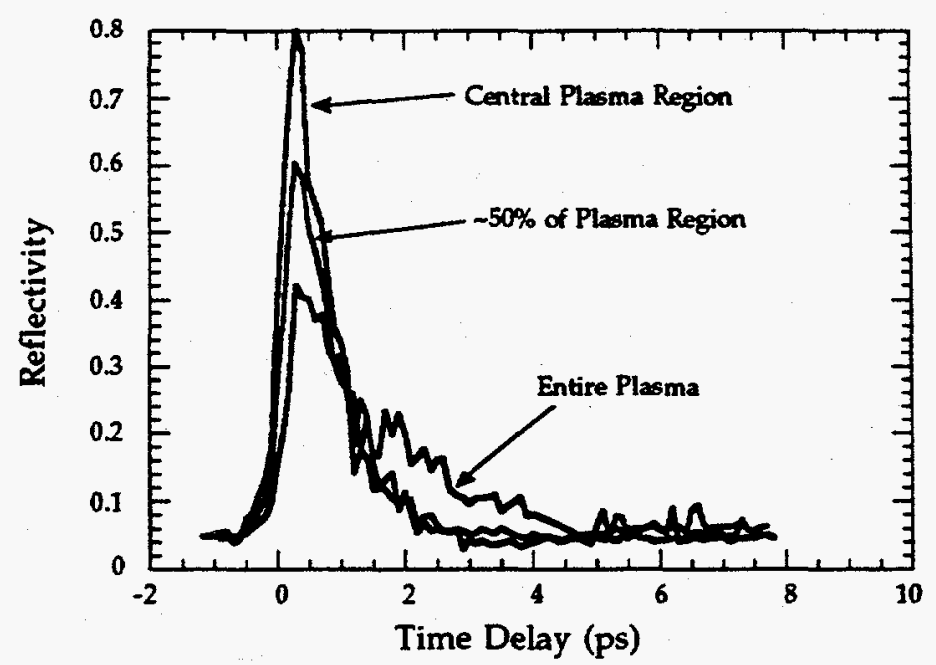

Figure 5.7: Reflectivity as a function of aperture size for a constant intensity $\left(-10^{16} \mathrm{~W} / \mathrm{cm}^{2}\right)$. The reflectioity increases and the relaxation time decreases as the aperture is closed down to image only the central portion of the plasma.

The spatial distribution of reflected intensities at the interaction region is the product of the incident intensity distribution at the focus and the intensitydependent reflectivity response. The intensity dependence of the reflectivity response for a pulse at the highest peak intensity $\left(I_{p} \sim 10^{16} \mathrm{~W} / \mathrm{cm}^{2}\right)$ is taken from a fit of the reflectivity data for $\mathrm{SiO}_{2}$ up to the highest intensity (Figure 5.8).

$$
R\left(I / I_{p}\right) \approx 1.0383-0.049027\left(I / I_{p}\right)+0.11461 \log \left(I / I_{p}\right)
$$

The intensity as a function of radius is given by the focal intensity distribution. The measured focal distribution is an Airy pattern because the unfocused spatial profile is nearly uniform. An Airy pattern closely resembles a Gaussian of the same FWHM, so we use the more tractable Gaussian. Using a normalized Gaussian intensity distribution $\left(I / I_{p}=\exp \left(-r^{2} / 4\right)\right)$ in the reflectivity data's functional fit, Eq. (5.1), we obtain an expression for the reflectivity distribution as a function of the radius $r$. 


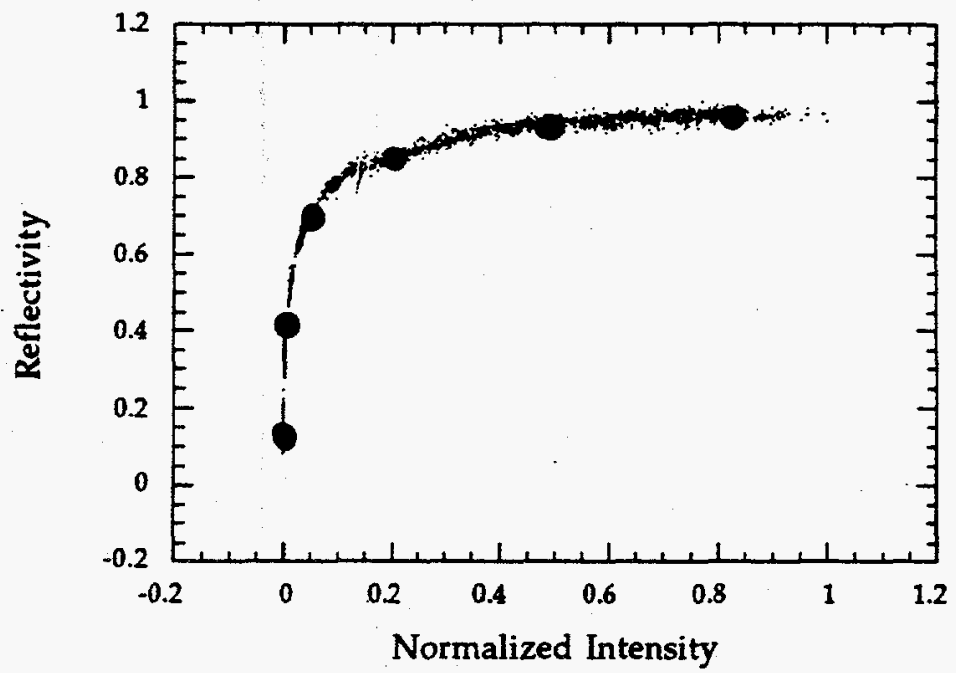

Figure 5.8: The reflectivity response at the highest peak intensity measured $\left(-10^{16} \mathrm{~W} / \mathrm{cm}^{2}\right)$ versus normalized intensity for $\mathrm{SiO}_{2}$ with S-polarization. Black circles indicate the fit to the data, Eq. (5.1).

$$
R(r) \approx 1.0383-\frac{0.049027}{e^{r^{2} / 4}}+0.11461 \log \left(e^{r^{2} / 4}\right)
$$

The product of Eq. (5.2) and the incident near-Gaussian focal intensity distribution gives the reflected intensity distribution. A magnified image of the reflected focal distribution at the interacton region is measured in the image plane located at the aperture and diode detector. In Figure 5.9, we plot the normalized distributions of the incident and reflected intensities as well as the plasma reflectivity versus the radial distance. The important feature is that at the highest intensity the reflected profile is nearly the same as the incident because the spatial reflectivity response does not change drastically over the focal region. This is equivalent to saying that at the highest intensity the reflected beam profile is nearly proportional to the incident profile because the reflectivity is nearly constant over a large range of intensities. Therefore, we can use a Gaussian intensity profile to estimate the reflectivity results as the aperture is changed. 
Note that this would not be true if the measurement of Figure 5.7 were taken at lower intensities. At lower intensities, the reflectivity response changes dramatically with intensity, Figure 5.8, and the reflected profile can therefore be significantly different than the incident profile.

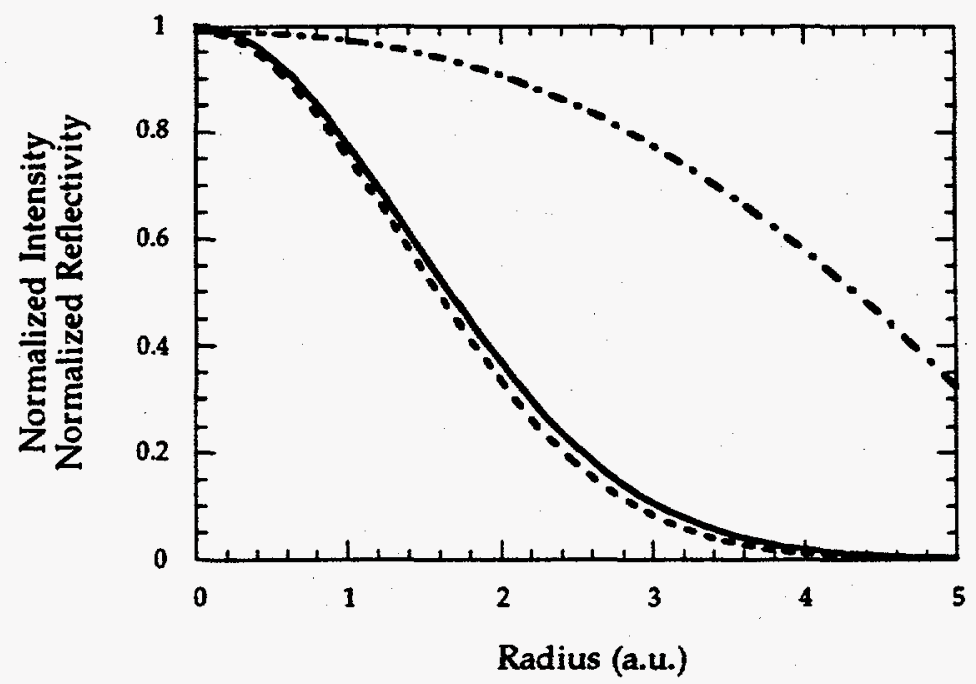

Figure 5.9: The incident (solid) and reflected (dash) intensity distributions and the reflectivity distribution (dot-dash) assuming the plasma response shown in Figure 4.2 (g) for $\mathrm{SiO}_{2}$, S-polarization.

The peak of a Gaussian is related to its average value by

$$
I_{\text {arg }}=\sqrt{\pi / 2} \operatorname{erf}(\sqrt{2}) I_{\text {peak }}=0.585 I_{\text {pak }}
$$

The highest measured reflectivity $(80 \%)$ and the spatially averaged (no aperture) reflectivity (42\%) shown in Figure 5.7 are related by a factor 0.525 , which is within $10 \%$ of that for a Gaussian. Therefore, the focal intensity distribution explains the distribution of plasma states, detected as different peak reflectivities, with reasonable accuracy.

This direct evidence of a spatial convolution of plasmas has important implications for plasma measurements. It indicates that the high-reflectivity and fast relaxation of the hotter central plasma can be masked by the lower average 
reflectivity and slower relaxation measured in a space-integrated measurement. Thus, for example, estimates from integrated data of absorption, electron heat conduction, $\mathbf{x}$-ray generation, hydrodynamics, and recombination can be significantly different from the estimates taken from the peak laser intensity. Electron heat conduction and hydrodynamics are investigated further with the spatially resolved measurements in the next section.

\subsubsection{Electron Heat Conduction and Plasma Hydrodynamics}

The extremely rapid reflectivity spike in the central plasma region, Figure 5.7, points to two important aspects of plasma dynamics -- electron heat conduction and hydrodynamic expansion. Reflectivity data shown in Figure 5.7 at $1 \times 10^{16} \mathrm{~W} / \mathrm{cm}^{2}$ from the central plasma region is duplicated in Figure 5.10. It shows a $0.57 \mathrm{ps}(\mathrm{fwhm})$ spike and two relaxation timescales, a fast $(\sim 100 \mathrm{fs})$ initial timescale and a second, slower timescale ( $>5 \mathrm{ps}$ ) which appears when the plasma reflectivity has relaxed to $\sim 10 \%$.

Probe reflectivity can decrease following the pump pulse interaction by three means: (1) electron heat conduction into the bulk target which rapidly cools the plasma and reduces the conductivity, (2) plasma cooling through hydrodynamic expansion, and (3) low-density hydrodynamic plasma expansion which obscures the highly reflective surface plasma, absorbing and scattering the probe pulse. We now investigate how the central plasma reflectivity data of Figure 5.10 may directly identify these processes.

The initial reflectivity relaxation rate is extremely fast with an undeconvolved falltime ( $90 \%-10 \%$ criteria) of $<100 \mathrm{fs}$. A clue as to what could cause such a rapid drop in the reflectivity may be found from a simulation of the temperature history of the plasma. Figure 5.11 is a simulation of the peak plasma temperature as a function of time for several incident pump intensities. This 
simulation, and additional simulation results presented later in this chapter, are derived from the hydrodynamic plasma code (LASNEX) mentioned in Section 4.3.3.4. Simulation inputs are an $\mathrm{SiO}_{2}$ target material with an S-polarized, 130fs, sech ${ }^{2}$ pulse at peak pump intensities of $10^{13} \mathrm{~W} / \mathrm{cm}^{2}$ to $10^{16} \mathrm{~W} / \mathrm{cm}^{2}$. The extended curve at $1016 \mathrm{~W} / \mathrm{cm}^{2}$ applies to the data shown in Figure 5.10. A 130fs sech 2 pulseshape is overlaid on the temperature curves to relate the temperature rates to the pulse timescale. The temperature history displays an initial $0.53 \mathrm{ps}$ ( $\mathrm{fwhm}$ ) temperature spike which relaxes in $\sim 100 \mathrm{fs}$ and a second slow temperature decrease over many picoseconds. Note that both the fwhm and falltime from the reflectivity data and initial temperature spike are in good agreement.

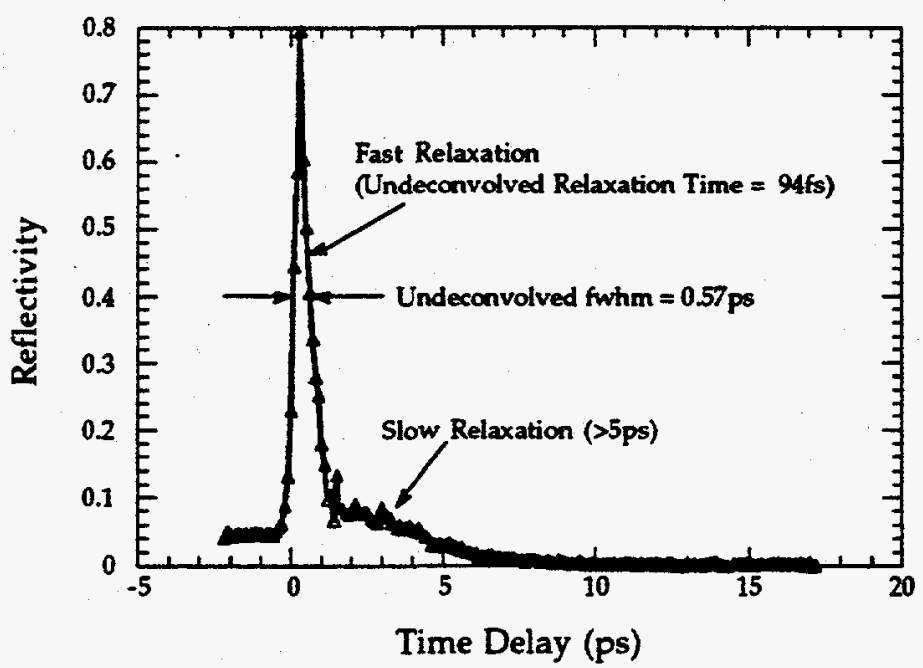

Figure 5.10: Reflectivity versus time in the central plasma region using an $\mathrm{SiO}_{2}$ target. Incident pump intensity is $1 \times 10^{16} \mathrm{~W} / \mathrm{cm}^{2}$. Probe is S-polarized at $30^{\circ}$ angle of incidence, pump is normally incident. 


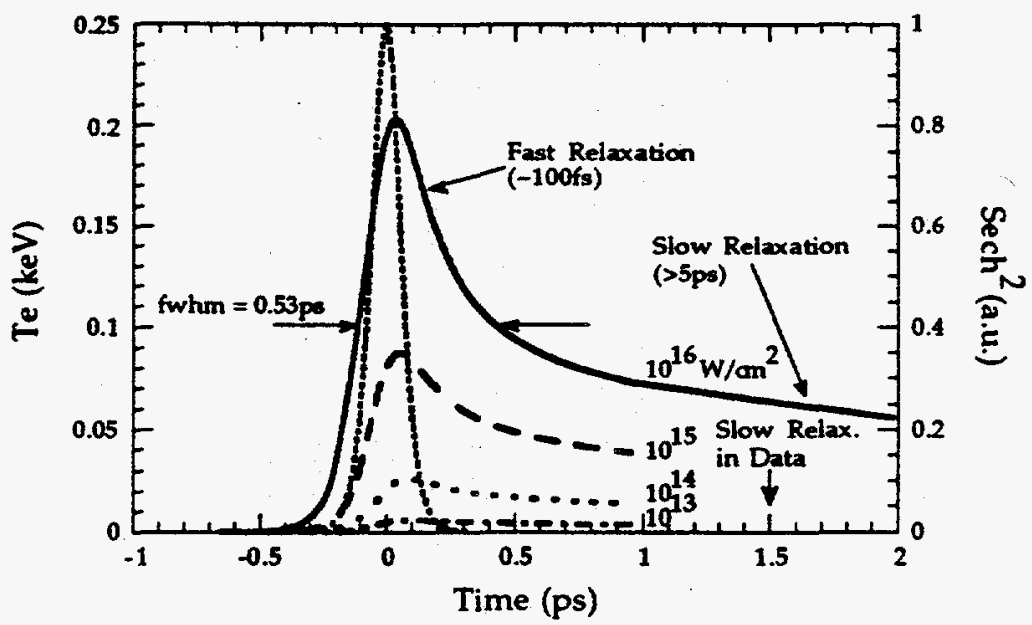

Figure 5.11: Temperature history simulation at pump intensities of $10^{16} \mathrm{~W} / \mathrm{cm}^{2}$ (solid), $10^{15} \mathrm{~W} / \mathrm{cm}^{2}$ (dash), $10^{14} \mathrm{~W} / \mathrm{cm}^{2}$ (dot), and $10^{13} \mathrm{~W} / \mathrm{cm}^{2}$ (dash-dot). $130 \mathrm{fs}$ fwhm sech $^{2}$ pulse (close dots) is shown for time reference.

More [172] identified the limiting factor determining both the magnitude and duration of the initial temperature spike as nonlinear thermal energy transport into the bulk material driven by electron heat conduction. The theory of nonlinear heat conduction is outlined by Zel'dovich [151]. Linear and nonlinear heat conduction in a fluid plasma model are derived from the energy balance equation

$$
\rho c_{p} \frac{\partial T}{\partial t}=-\nabla \cdot S+W
$$

where $\rho$ is the density, $c_{p}$ is the specific heat at constant pressure, $S$ is the heat flux vector, and $W$ is the power density released from external sources. In the linear approximation, heat flux is proportional to the temperature gradient

$$
S=-\kappa \nabla T
$$

where $x$ is the coefficient of thermal conductivity, which depends on the state and properties of the plasma. In the linear approximation, $x$ is treated as a constant and a linear heat conduction equation is derived 


$$
\frac{\partial T}{\partial t}=\chi \nabla^{2} T+q
$$

where the coefficient of thermal diffusivity $\chi=\kappa / \rho c_{p}$ and $q=W / \rho c_{p}$. At temperatures of several $\mathrm{eV}$ or higher the coefficient of thermal conductivity becomes temperature dependent and the heat conduction equation is nonlinear

$$
\frac{\partial T}{\partial t}=\nabla \cdot(\chi \nabla T)+q
$$

The thermal diffusivity coefficient can often be approximated as proportional to a power of the temperature, $\chi=a T^{n}$, giving a nonlinear equation of the form

$$
\frac{\partial T}{\partial t}=a \nabla \cdot\left(T^{n} \nabla T\right)+q
$$

For electron heat conduction $\chi_{e} \sim T_{e}^{5 / 2}$ [151]. As plasma is formed, an extreme temperature gradient grows between hot surface plasma and colder, unionized bulk material. Since the electron conduction rate depends primarily upon this temperature gradient, Eq. (5.8), extremely fast conduction rates can be reached which both limit the peak temperature and drive the rapid cooling of the initial temperature spike in Figure 5.11. Simulations of peak electron temperature and the electron density versus space are shown in Figures 5.12 and 5.13, respectively. Peak electron temperature decreases from $\sim 200 \mathrm{eV}$ to $\sim 50 \mathrm{eV}$ during the initial temperature spike. At the same time, electron density in the first 1000 angstroms before the target surface drops by a factor of three. Note in Figure 5.12 how electron transport drives a nonlinear heat wave several thousand angstroms into the target. 


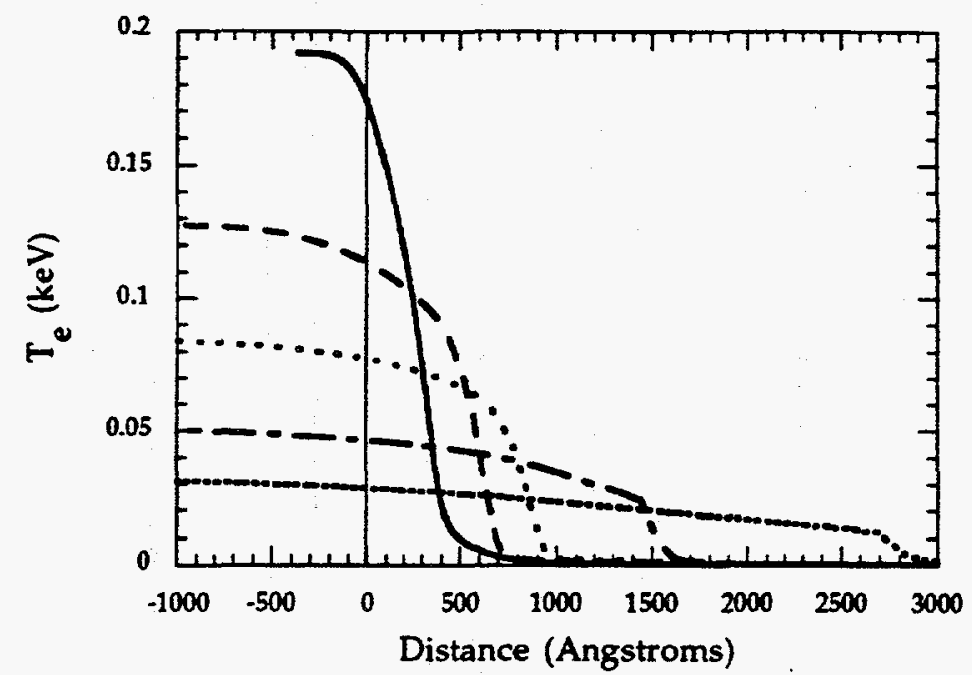

Figure 5.12: Peak electron temperature versus distance at the peak of the pulse (solid), and at 0.22 (dash), 0.54 (dot), 1.70 (dash-dot), 4.42 (close dots) picoseconds after the peak. The original target surface is indicated by a the line at 0 angstroms and the bulk target is to the right.

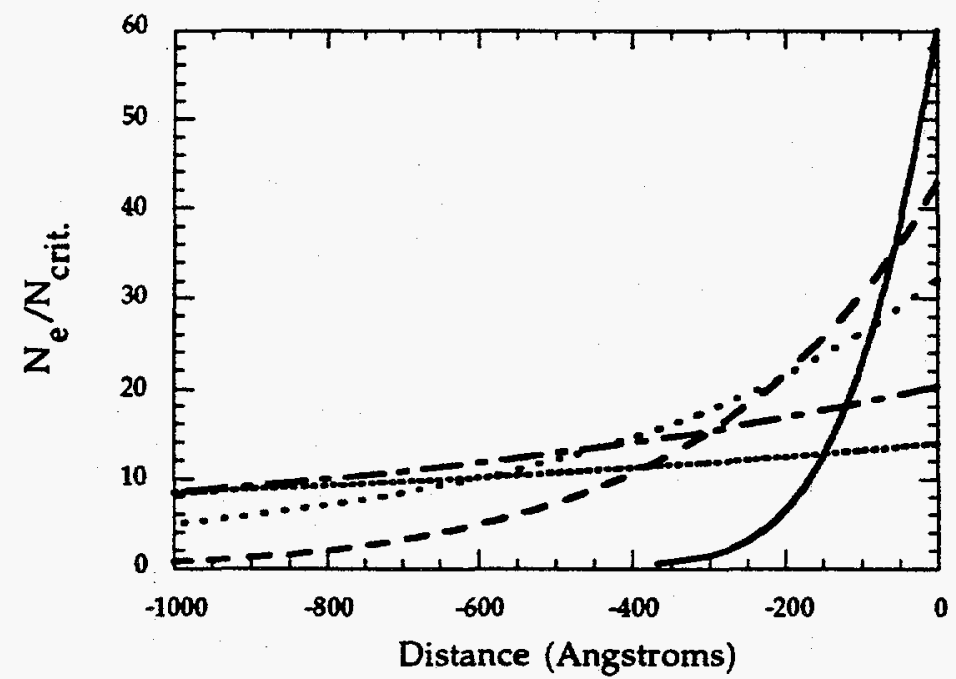

Figure 5.13: Electron density normalized to critical density $\left(2.92 \times 10^{21} \mathrm{~cm}^{-3}\right)$ versus distance at the peak of the pulse (solid), and at 0.22 (dash), 0.54 (dot), 1.70 (dash-dot), 4.42 (close dots) picoseconds after the peak. The original target surface is located at 0 angstroms. 
The timescale for the thermal conduction wave to propagate into the material has been measured at a lower intensity, $5 \times 10^{14} \mathrm{~W} / \mathrm{cm}^{2}$, by $\mathrm{Vu}[173]$. Time-resolved spectral blue-shifting of an optical probe on the target's backside was attributed to Doppler shift in the reflected pulse due to a moving thermal wave. At an incident pump intensity of $5 \times 10^{14} \mathrm{~W} / \mathrm{cm}^{2}$ spectral shifts indicated a supersonic thermal wave producing significant shifts for $\sim$ 1ps. At our intensities we expect an even shorter conduction timescale, because the rate depends on the temperature gradient which is significantly steeper at $1016 \mathrm{~W} / \mathrm{cm}^{2}$. Backside probe reflectivity data [173], on the other hand, had a 1/e falltime of about 15ps. Vu's spectral shifting and reflectivity data indicate that a burst of heated electrons drives a thermal wave into the cold bulk and quickly ( $1 \mathrm{ps})$ dies out leaving behind a hot plasma region in the bulk which remains supercritical until slower recombination, cooling and diffusion mechanisms quench the reflectivity. The behavior is in general agreement with the theory of nonlinear heat conduction [151] and Figure 5.12. A nonlinear heat wave propagates supersonically, a finite distance into the material with a sharply defined boundary between heated and cold regions. The important point to our experiment is backside spectral shifting measurements confirm our frontside subpicosecond timescale for electron heat conduction as inferred by the initial fast reflectivity decrease.

Hydrodynamic expansion during the first few hundred femtoseconds must also be considered as a source for cooling which could lead to the initial fast reflectivity drop. Simulations, however, show that the total plasma energy lost to hydrodynamics in the first few hundred femtosecond is only about $10 \%$ of the energy lost through electron conduction. Thus, plasma expansion cooling only accounts for a small part of the initial rapid temperature decrease while electron conduction cooling comprises the remainder. 
The source of the second, slower, rate in the temperature history of Figure 5.11 was investigated by turning off the hydrodynamic expansion in the simulation. The result is shown in Figure 5.14. While hydrodynamics clearly reduced the peak temperature, neither the fast nor the slow cooling timescales were significantly changed. Electron conduction thus controls both the fast and slow cooling rates. It would seem that we could therefore explain both reflectivity relaxation rates in Figure 5.10 by the parallel rates due to electron conduction. However, we must also consider the effect of hydrodynamics on probe reflectivity.

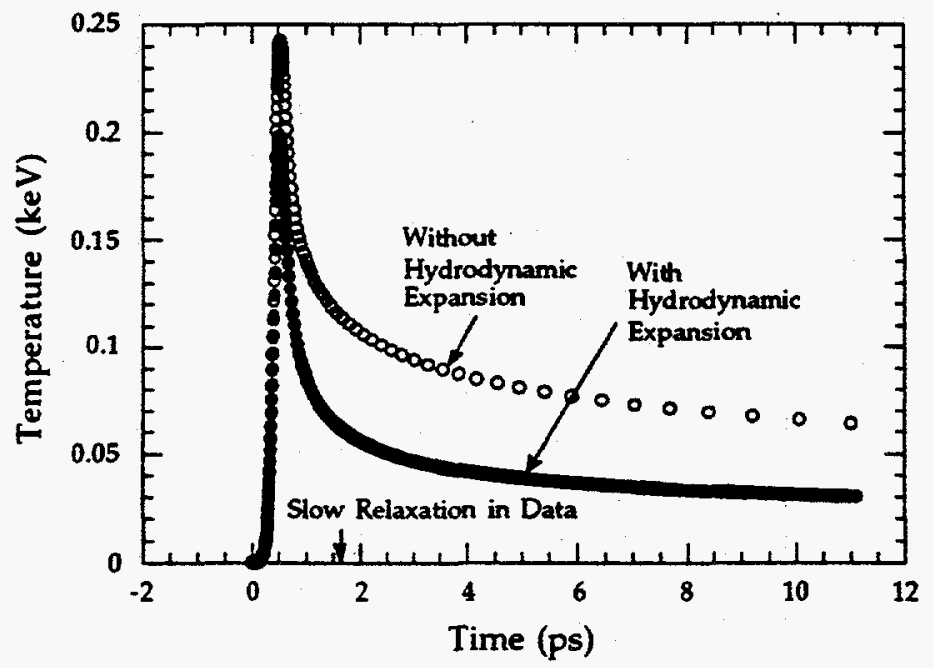

Figure 5.14: Peak plasma temperature versus time with and without hydrodynamic expansion.

Reflectivity several picoseconds after the pulse interaction is determined primarily by absorption in an intensity-dependent hydrodynamic plasma expansion. This is an important departure from the "surface" measurements made during the pulse interaction and before significant expansion has occurred. Separation of reflective probing measurements into two distinct regimes, (1) dense plasma and (2) hydrodynamic effects, was recognized by Heremans [174] 
and unambiguously established by Reitze [4]. Wang [167] used a hydrodynamic surface expansion model to successfully explain reflectivity recovery in metals excited by 90 fs pulses at $\sim 10^{13} \mathrm{~W} / \mathrm{cm}^{2}$. Landen $[116,134,175]$ has directly measured hydrodynamic expansion with a $1 \mathrm{ps}, 10^{14} \mathrm{~W} / \mathrm{cm}^{2}$ pulse using resonance absorption spectroscopy and spectral shifts. Here, we extend hydrodynamic measurements to intensities two to three orders of magnitude beyond those of Reitze, Wang and Landen. During the pulse interaction, the reflectivity's intensity dependence arises from a balance between several effects as the intensity increases: (1) increasing temperature and density (higher conductivity) from laser heating, (2) decreasing temperature and density from electron conduction and hydrodynamic expansion (lower conductivity), and (3) increasing scale length (more absorption). Time-integrated reflectivity curves presented in Chapter 4, which only measure dynamics occurring during the pulse interaction, reflect these competing effects at moderate intensities $\left(\sim 10^{13}\right.$ $10^{15} \mathrm{~W} / \mathrm{cm}^{2}$ ). At both lower and higher intensities, other mechanisms affect the absorption, as discussed in Chapter 4.

Reitze [4], however, identified a critical time window of 1-2ps following excitation in graphite with $90 \mathrm{fs},<2.2 \times 10^{14} \mathrm{~W} / \mathrm{cm}^{2}$ pulses during which surface plasma states could be probed without significant interference from hydrodynamic expansion. At the higher intensities used here (up to $\sim 1016 \mathrm{~W} / \mathrm{cm}^{2}$ ), we expect this time window to be reduced by faster plasma expansion. The best evidence for this is the improved results obtained, in Chapter 4 and by other authors, by using Helmholtz wave equations rather than a Fresnel (zero scale length) analysis. We assert, however, that although some interference from hydrodynamic expansion always occurs at large intensities, electron conduction cooling is measurable and is the dominant mechanism initially lowering the reflectivity. Within a narrow time window, absorption from ablated plasma is small, 
while absorption from conduction cooling is large. After the significant electron conduction dies out, a slower drop in reflectivity shows absorption dominated by hydrodynamics.

Plasma expansion is generally considered $[3,176,177]$ to create a strong reflectivity decrease when the normalized scale length $L / \lambda>0.1$. This criteria also depends to some extent on the polarization, wavelength, material, and density gradient. A simulation of the electron density scale length and temperature, is shown in Figure 5.15. Scale length is limited to $L<0.1 \lambda=614 \AA$ in the first 2.5 ps, so reflectivity is not severely affected by expanded plasma. More importantly, this means it is possible to probe electron conduction in this critical time window. Note that the significant electron conductivity measured by $\mathrm{Vu}$ [173] lasted for only about 1 ps. On the other hand, beyond approximately 2.5 ps $L>0.1 \lambda$ and reflectivity is strongly reduced by absorption and scattering in the underdense plasma.

In addition, note how the temperature history drives the plasma expansion timescales in Figure 5.15. The initial temperature spike in the first picosecond drives a rapid initial expansion out about $300-400 \AA$ from the target surface. A simulation of absorption in the hydrodynamic scale length, Figure 5.16 , shows this initial expansion ( $0-1 \mathrm{ps})$ is not large enough to cause significant absorption. As the second temperature timescale in Figure 5.15 appears, expansion slows considerably. Since the discussion above indicates how both temperature timescales are associated with electron heat conduction, expansion behavior is simply another manifestation of electron conduction cooling. It reduces the thermal pressure gradient and controls the expansion rates. Thus, the change in the scalelength timescales is the frontside probe analogue to the backside electron heat conduction spectral shifting probe observed by $\mathrm{Vu}$. Both show the conduction disappears at approximately the same time, $\sim 1 p s$. Because 
the initial fast expansion scalelength is not enough to cause a severe reflectivity drop, the expansion simulation is further evidence that it is cooling by electron conduction, and not expansion absorption, which causes the majority of the initial rapid relaxation rate in the reflectivity. Moreover, the simulation of absorption in the hydrodynamic scale length, Figure 5.16, clearly displays the absorption timescale ( $\sim 5-10 \mathrm{ps})$ as comparable to the second, slow ( $>5 \mathrm{ps})$, timescale seen in the reflectivity data.

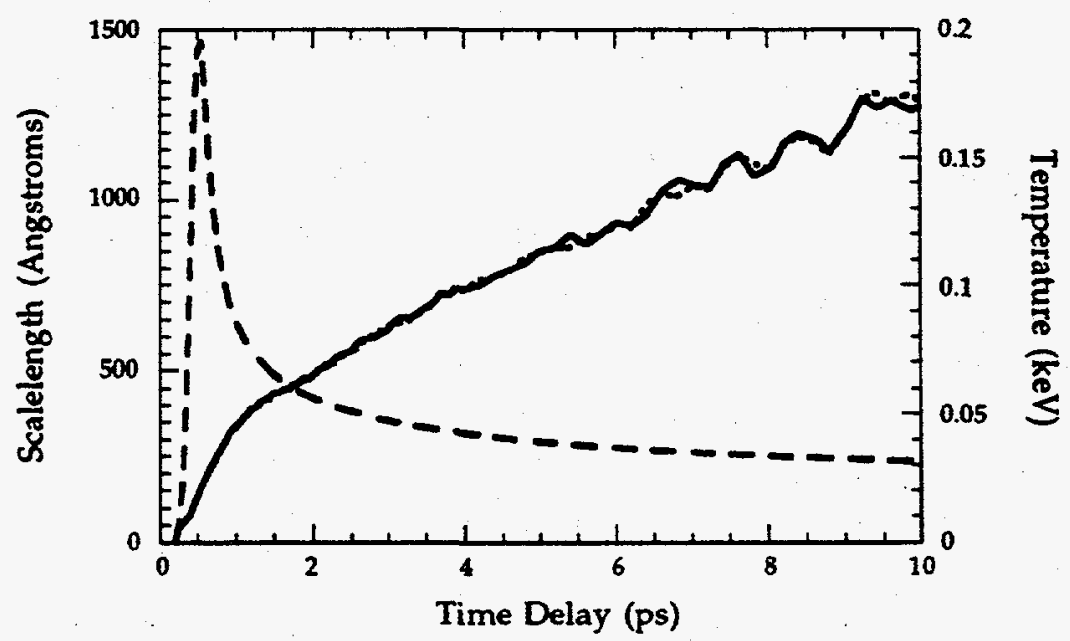

Figure 5.15: Simulation of the plasma scalelength for $\mathrm{SiO}_{2}$ target at $1 \times 10^{16} \mathrm{~W} / \mathrm{cm}^{2}$. Scalength is calculated with (solid) and without (dot) the pondermotive force. Pondermotive force is discussed below. Peak electron temperature (dash) with and without the pondermotive force is shown for reference. Note, electron temperature is not changed by the pondermotive force.

Another consideration is pondermotive light pressure which suppresses plasma expansion at high intensities, making electron conduction easier to probe. Liu [178] has studied the competition between the thermal pressure gradient causing expansion and the pondermotive pressure. Plasma expansion was found to be significantly slowed when the average energy of the electron quiver velocity in the light field, $m v_{o s c}^{2} / 2$, is about the same or greater than the electron 


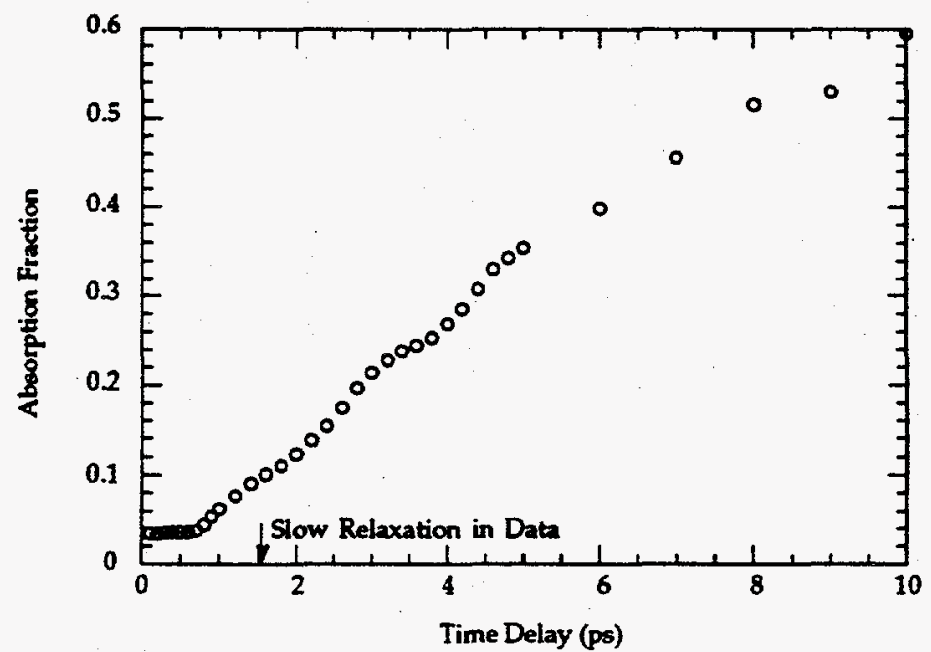

Figure 5.16: Plasma absorption in the hydrodynamic scale length. Absorption becomes large only after significant expansion occurs ( $>1 p s$ ). Absorption timescale is comparable with the slow relaxation of the reflectivity data shown in Figure 5.10 .

thermal energy, $k T_{e}$. This condition can be approximately expressed by the relation [178]

$$
m\left\langle v_{o s c}^{2}\right\rangle / 2 k T_{c} \equiv 3.2 \times 10^{-13} I_{i n c} \lambda_{0}^{2} / k T_{e} \geq 1
$$

where $I_{i n c}$ is the incident intensity in $W / \mathrm{cm}^{2}$ and $\lambda_{0}$ is the vacuum wavelength in microns, and $k T_{e}$ is the electron temperature in $\mathrm{eV}$. In our experiment, at an intensity of $10^{16 \mathrm{~W}} / \mathrm{cm}^{2}$, the ratio is about $6\left(\lambda_{0}=0.614 \mu \mathrm{m}, k T_{e}=200 \mathrm{eV}\right)$, well above the level at which pondermotive pressures should become important. Using time-integrated measurements of Doppler-shifted spectra at an incident intensity of $2 \times 10^{15} \mathrm{~W} / \mathrm{cm}^{2}$ in a $1 \mathrm{ps}$ pulse, Liu found the scale length was reduced by a factor $\sim 3$ due to pondermotive pressure. At our intensities, we expected pondermotive scale length suppression to be even more dramatic. Thus, at intensities $-10^{16} \mathrm{~W} / \mathrm{cm}^{2}$ the time before significant expansion would be both reduced below the $1-2$ ps seen previously at $10^{14} \mathrm{~W} / \mathrm{cm}^{2}[4]$ and increased by 
pondermotive pressure. Detailed simulations with and without pondermotive pressure, however, did not show a strong retardation of the expanding scalelength (Figure 5.15). This may be an indication that the pondermotive pressures are important only for significantly longer pulses where the scale length is large, as in Liu's experiment (a 1ps pulse), or at ultra-high intensities $\left(>10^{16} \mathrm{~W} / \mathrm{cm}^{2}\right)$ with ultra-short pulses. Nevertheless, using free expansion alone, we expect a time window for unexpanded plasma wide enough for our identification of both electron conduction and hydrodynamics.

Finally, let us now consider if it is possible that other hydrodynamic expansion effects could cause both the initial fast and the second slow timescale. First, two timescales could be the result of radial and axial, two-dimensional, plasma expansion. A density reduction by significant radial plasma expansion at the transition time might explain the results. Second, it can be driven by a macroscopic change in the hydrodynamic expansion geometry, another twodimensional expansion effect. When plasma expands beyond twice the radius of the focal plane, absorption and scattering can change, because the geometry evolves from planar to nearly spherical [94]. Third, the fast rate could indicate plasma expansion with low recombination rates then, as the expansion cools, recombination becomes significant which introduces the slow timescale data.

We can eliminate the first two possibilities immediately. The hydrodynamic expansion is considered to be roughly planar if the expansion length $c_{s} \tau$ is such that [94]

$$
c_{s} \tau<2 R_{\text {focal spot }}
$$

where $c_{s}$ is the plasma sound speed, $\tau$ is the expansion time and $R_{\text {focal spot }}$ is the radius of the focal spot. The plasma expands at $c_{s} \sim 10^{7} \mathrm{~cm} / \mathrm{s}$ and the focal spot is $R_{\text {focal spot }} \sim 10 \mu \mathrm{m}$. Eq. (5.10) then yields a time for transition from planar to spherical geometry of $\sim 200 \mathrm{ps}$, more than two orders of magnitude greater than 
the observed time for a change in the reflectivity timescales, $\sim 2$ ps. Moreover, completely axial expansion is a good approximation when the critical density surface moves less than a few percent of the spot size. In $\sim 2 p s$, simulations show the critical surface motion to be about $5000 \AA$, which is only $3 \%$ of the measured $15 \mu \mathrm{m}$ spot size. No experimental of theoretical evidence for two-dimensional effects has been shown to date. Finally, the third possibility, recombination, is unlikely under our conditions [179]. Therefore, expansion effects cannot account for both the slow and the fast reflectivity timescales.

An additional test for the identification of electron conduction and hydrodynamics was performed using target materials with similar optical properties, but significantly higher impurity concentrations. Large impurity concentrations are more readily ionized by the laser within the skin depth and by heated electrons in the bulk material. As a result, a larger underdense plasma with smaller temperature gradients is formed in the bulk material. Backside probing [180] experimentally verified the enhanced bulk plasma. Since electron heat conduction depends strongly on the temperature gradient, we tested the sensitivity of our electron conduction measurement by inducing a lower temperature gradient with impurities. If the conduction rate was slow and the plasma remains hot due to impurities, the initial rapid drop in the reflectivity should not appear as it did with the very pure fused quartz target results of Figure 5.10.

The impurities in the targets tested ranged over nearly every common element ( $\mathrm{Al}, \mathrm{Ca}, \mathrm{Cu}, \mathrm{Fe}, \mathrm{Pb}, \mathrm{Mg}, \mathrm{K}, \mathrm{Na}, \mathrm{Ti}, \mathrm{Li}, \mathrm{Mn}, . .$. ) and many common compounds $\left(\mathrm{Na}_{2} \mathrm{O}, \mathrm{K}_{2} \mathrm{O}, \mathrm{PbO}, \mathrm{OH}, \ldots\right)$ in varying percentages. Impurity percentages, by weight, are approximately: $<0.02 \%$ for fused quartz, $\sim 28 \%$ for glass, and $\sim 50 \%$ for lead glass. Reflectivity results for the central plasma region in each material are shown in Figure 5.17. The reflectivity relaxation time grows 


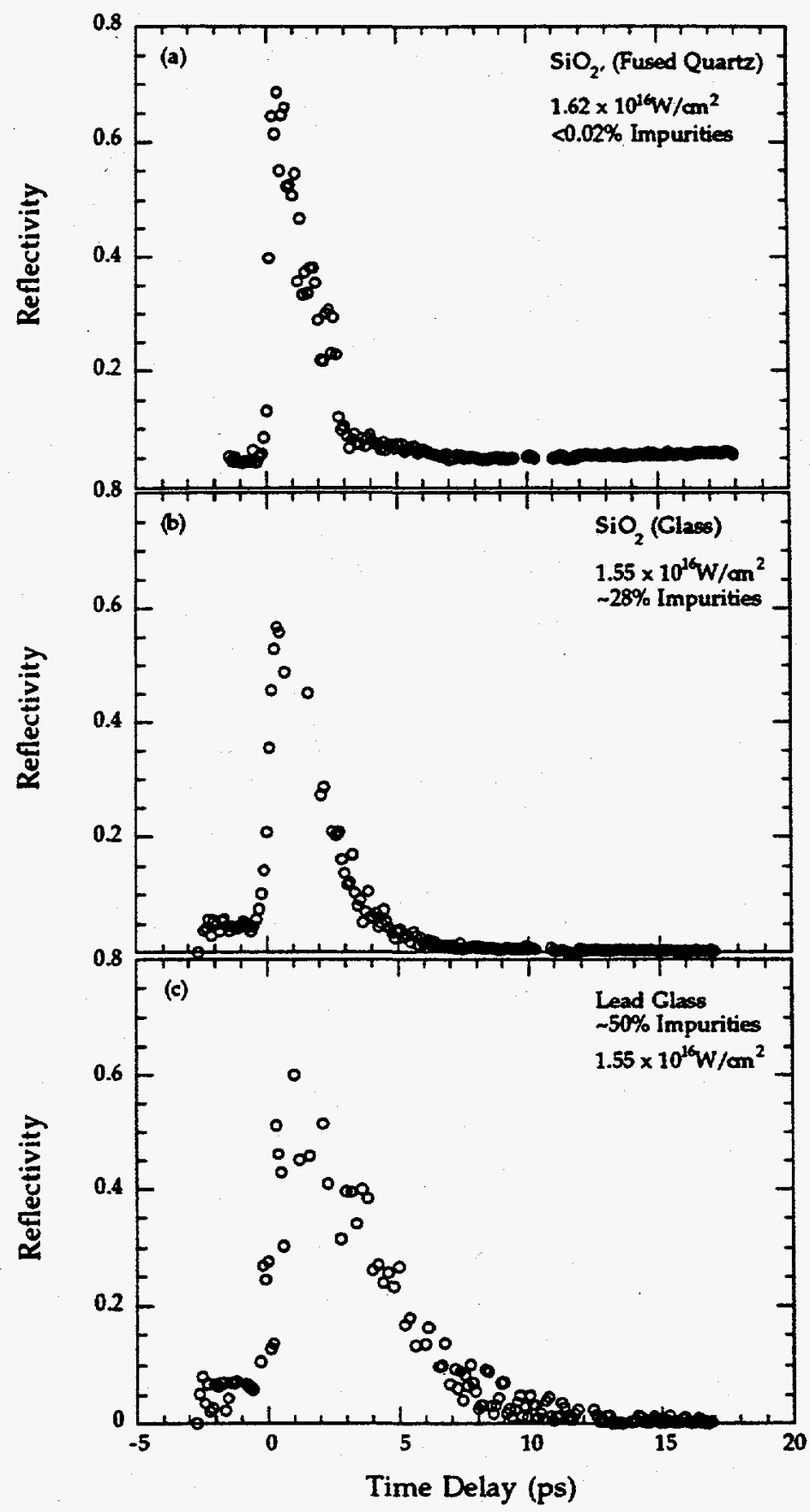

Figure 5.17: Reflectivity in the central plasma region versus time delay for (a) $\mathrm{SiO}_{2}$ (fused quartz), (b) $\mathrm{SiO}_{2}$ (glass), and (c) lead glass.

significantly to $\sim 5 \mathrm{ps}$ for the glass target and $\sim 10 \mathrm{ps}$ for the lead glass target. These timescales are well beyond the initial rapid electron conduction rate and appear to indicate only absorption in the underdense hydrodynamic expansion. 


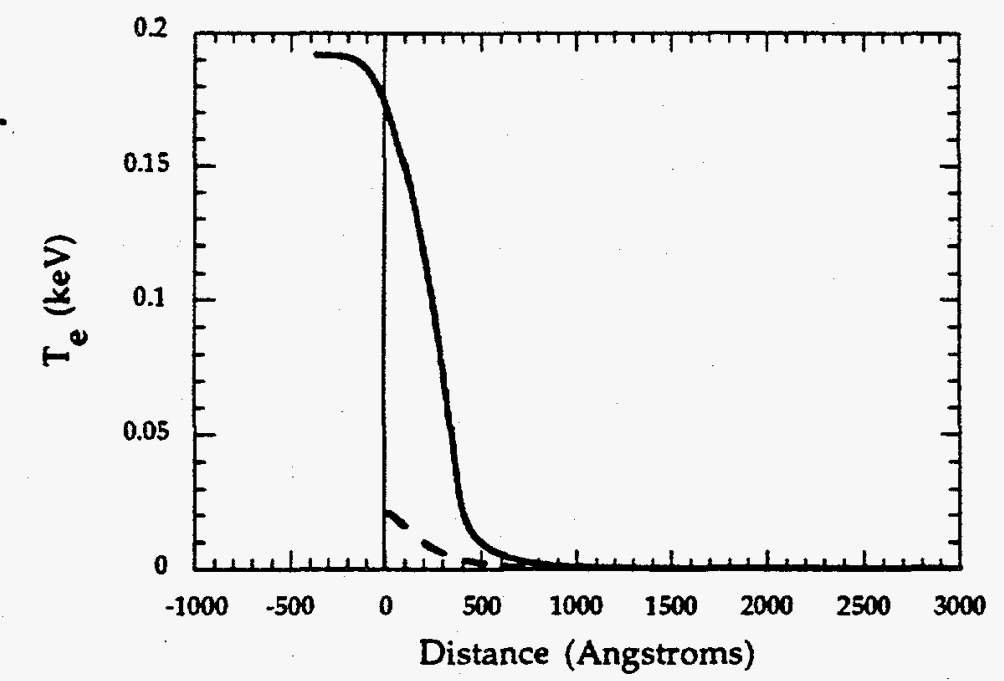

Figure 5.18: Comparison of the gradients in the peak electron temperature at the peak of the pump pulse for intensities of $10^{16} \mathrm{~W} / \mathrm{cm}^{2}$ (solid) and $10^{14} \mathrm{~W} / \mathrm{cm}^{2}$ (dash).

Unambiguous identification of electron thermal conduction followed by plasma expansion seems to demand a considerable temperature gradient to drive the heat conduction faster than the plasma expansion obfuscation. This is the reason it has not been seen in previous frontside probe measurements. Reitze [4] and $\mathrm{Vu}$ [173] used intensities nearly two orders of magnitude below those applied here. As a result, the temperature gradients were not large enough to drive rapid plasma cooling by electron conduction, as shown in Figure 5.18. In fact, in this experiment the effects of electron conduction were seen only in the center of the plasma at intensities above $\sim 5 \times 10^{15} \mathrm{~W} / \mathrm{cm}^{2}$ (Figure 5.19). Below this intensity, the reflectivity relaxation timescale grew to $>5 \mathrm{ps}$ and agreed with the hydrodynamic timescale found by Reitze and $\mathrm{Vu}$. Evidence of electron conduction should be seen at intensities higher than this work, however, this demands an exceptionally low prepulse and steeply rising main pulse to avoid interference from significantly faster scalelength formation. Unless this ultra- 
clean pulse is achieved, it is expected that direct evidence identifying two distinct regimes, electron conduction and hydrodynamics, will be limited to the rather narrow region of intensities used in this experiment.

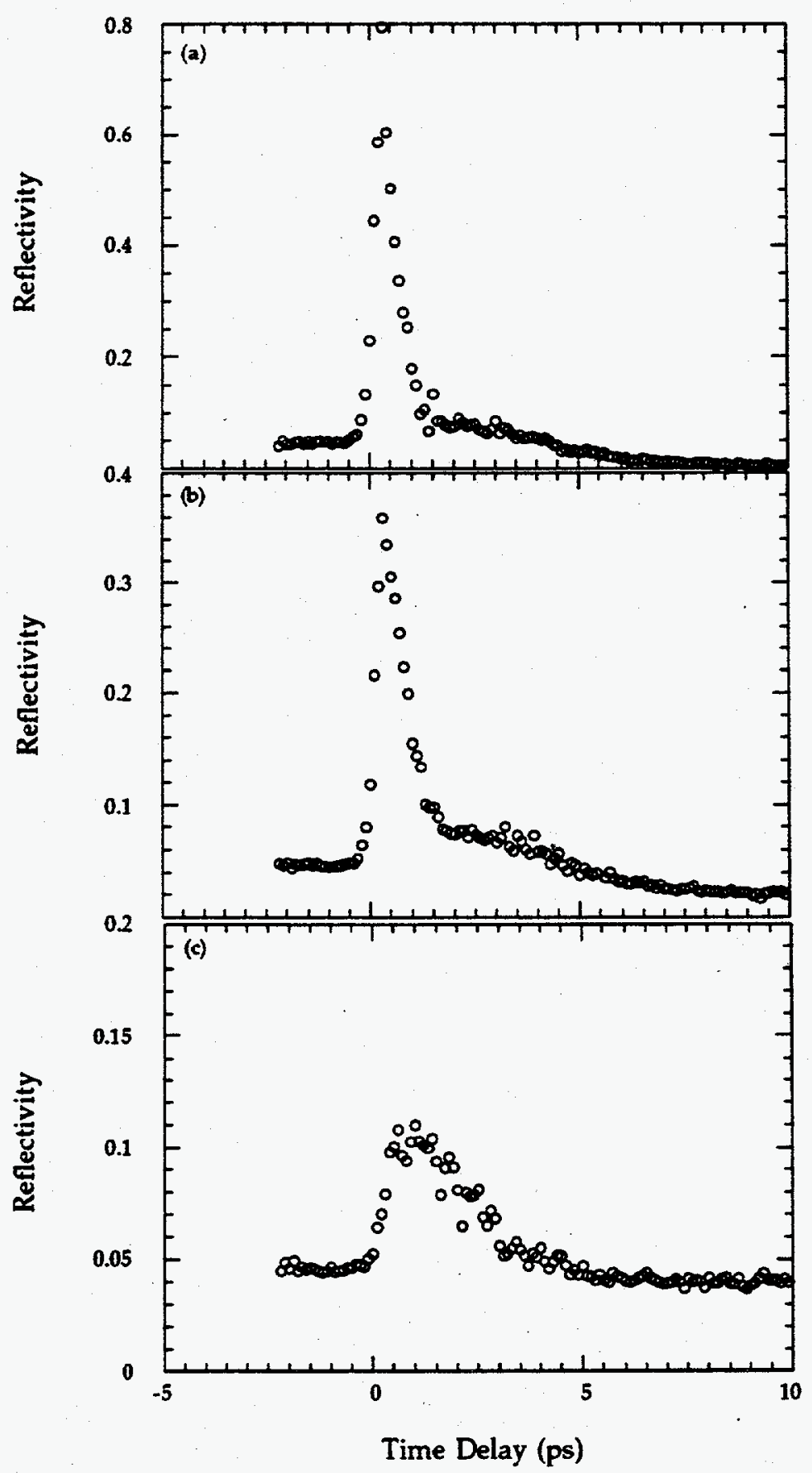

Figure 5.19: Reflectivity versus time delay in the central plasma region for intensities of (a) $1 \times 10^{16} \mathrm{~W} / \mathrm{cm}^{2}$, (b) $6 \times 10^{15} \mathrm{~W} / \mathrm{cm}^{2}$, and (c) $3 \times 10^{13} \mathrm{~W} / \mathrm{cm}^{2}$ 


\section{CHAPTER 6:}

\section{THE PLASMA MIRROR}

\subsection{INTRODUCTION}

The plasma mirror is a simple application of ultra-short pulse plasmas for ultra-fast light switching at optical wavelengths. The rapid change in reflectivity of an insulating target upon critical density plasma formation can be used to switch away prepulse energy. Prepulse energy in amplified, ultra-short pulse systems arises from amplified spontaneous emission in high-gain amplifier stages, spectral cutoff in fiber and grating pulse compression techniques, and spectral distortion in CPA systems. A final research goal was to make direct measurements on this plasma-based device for use as a prepulse suppresser in laser systems with extremely high intensities $\left(>10^{18} \mathrm{~W} / \mathrm{cm}^{2}\right)$, where a high contrast ratio is critical to studying high-density plasmas.

Several attributes make an ultra-short pulse plasma a natural candidate for use as an ultra-fast switch for optical beams. First, the plasma reaches critical, i.e., "switching," density on a femtosecond timescale. Second, high electron densities make it possible to switch all radiation up to the UV spectral region ( $\mathrm{n}_{\text {crit }}=10^{23} \mathrm{~cm}^{-3}$ implies $\lambda \sim 0.1 \mu \mathrm{m}$ ). Third, the plasma geometry resembles a flat mirror because little hydrodynamic expansion occurs during the pulse interaction. The reflected pulse is expected to be spatially and temporally clean with little phase distortion, retaining the incident pulse's propagation and focusing properties. Fourth, the plasma mirror can be used in a simple, selfinduced configuration where the reflectively switched pulse also creates the plasma switch. This is only possible because the plasma formation mechanism is tunnel ionization which can occur within one optical cycle on the pulse's rising 
edge. Longer pulse plasmas form by avalanche ionization on the timescale of the entire pulsewidth, not during the pulse's rising edge. The self-induced feature contrasts with semiconductor switching techniques which use short pulses to create carriers and induce reflective gating on a second, longer pulse [181, 182].

Alternative prepulse suppression methods have been studied, because it is essential for many experiments with high-intensity lasers. The plasma mirror has advantages over some of these methods. One alternative is second harmonic generation of the amplified light [183]. Since second harmonic generation depends on the square of the intensity, its contrast ratio should, ideally, be improved to the square of it's value in the fundamental beam. In addition, the main pulse shape should sharpen somewhat due to the harmonic generation process [183]. A sharper pulseshape is better for creating high-density plasmas because experiments rely on a fast rise time so energy is deposited before plasma expansion begins. In Section 6.6 we show that the plasma mirror is also predicted to decrease the pulse risetime. The drawback of the second harmonic technique is the expense of buying a large, optical quality nonlinear crystal. A large crystal is necessary because a high-intensity pulse must be spread out over a large area to keep fluence below the crystal damage level. Another alternative, nonlinear optical fiber techniques, $[184,185]$ cannot be used for prepulse suppression after the pulse is amplified to its highest energy and compressed to its shortest duration because the light is above the fiber damage threshold. Prepulse may be produced in the final compression or amplification stages, so the nonlinear fiber technique would not be entirely useful even if it were effective in stretching and pre-amplification stages. The plasma mirror, on the other hand, is most effective at the final compressed, high-energy stage, because plasma is created most easily. 
Techniques to correct or eliminate phase distortions directly at the prepulse source in CPA systems include spectral windowing [186-188], temporal windowing [47], and phase correcting pulse stretchers [189]. As discussed in Section 2.6.1.2, SPM in the fiber used for pulse stretching in some CPA systems produces a pulse with a nearly linear, positive chirp in the center. The pulse's linear portion can be compressed by a dispersive element such as a grating pair. Nonlinear and negatively chirped portions will not be compressed and result in a low-intensity, picosecond to nanosecond pedestal around the main pulse. The chirped pulse figure in Chapter 2, Figure 2.9, is duplicated in Figure 6.1 with the effects of spectral and temporal windowing.

Spectral windowing uses spatial apertures or gain narrowing to filter high and low frequency pulse components that have the most nonlinear chirp. As shown in Figure 6.1, spectral windowing leaves negatively chirped portions unfiltered. The negatively chirped portions expand in a grating or prism compressor to form a low energy pedestal. A contrast ratio of 700 has been reported [187] with this technique. Temporal windowing uses a fast saturable absorber, or two fast Pockels cell switches, to select only the chirped pulse's temporally centered (linear) part. Using the saturable absorber a contrast ratio of 900 was reported [190] and using the Pockels cells a contrast ratio of better than $10^{7}$ was reported [47]. Finally, a pulse stretcher with continuously variable and independent adjustment of the second, third, and fourth order frequencydependent phase was under investigation at the time of this writing [189]. The pulse stretcher incorporates a specially designed air-spaced, achromatic doublet lens. Adjustments can be made to the lens distance, and lateral and longitudinal lens spacing for second, third, and fourth order phase corrections, respectively. The stretcher is designed to allow nonlinear phase pre-compensation that a 
linearly dispersive grating compressor cannot correct. This technique had not been experimentally tested at the time of this writing.
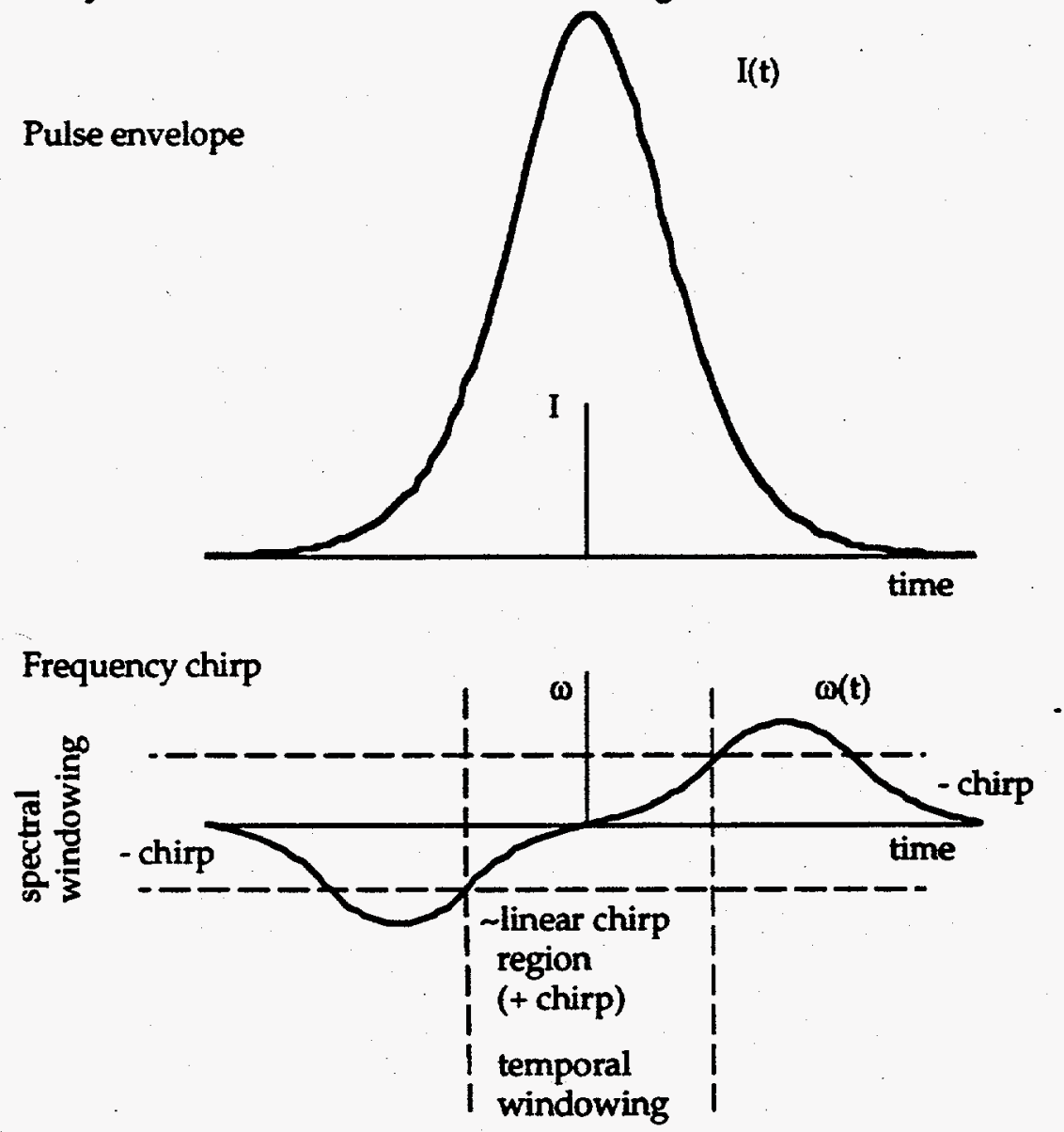

Figure 6.1: Temporal and spectral windowing on a chirped pulse.

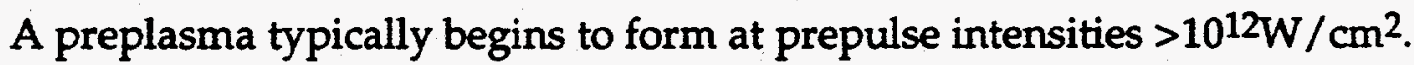
The best measured contrast ratio for the ultra-high-intensity, solid-state laser systems are typically measured to be $<10^{6}$, with considerable attention to the

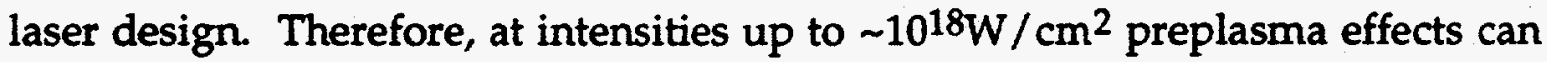
largely be avoided. At the higher intensities, $>1020 \mathrm{~W} / \mathrm{cm}^{2}$, projected for the near future, severe prepulse levels will make it impossible to design experiments to study solid-density plasmas with relativistic electrons and copious amounts of hard x-ray emission. Therefore, a prepulse suppresser is a critical component for the available laser system technology and anticipated power levels. 


\subsection{THE PLASMA MIRROR SCHEME}

In the plasma mirror scheme, Figure 6.2, a high-intensity, ultra-short laser pulse is focused onto a solid (insulator), initially transmissive target. The laser pulse is focused so prepulse intensity is below the target ionization and damage threshold and the intensity, at some point during the main pulse, is above the critical ionization threshold. The main pulse abruptly initiates a plasma and drives it to a highly reflective critical or supercritical state with a characteristic nonlinear intensity dependence.

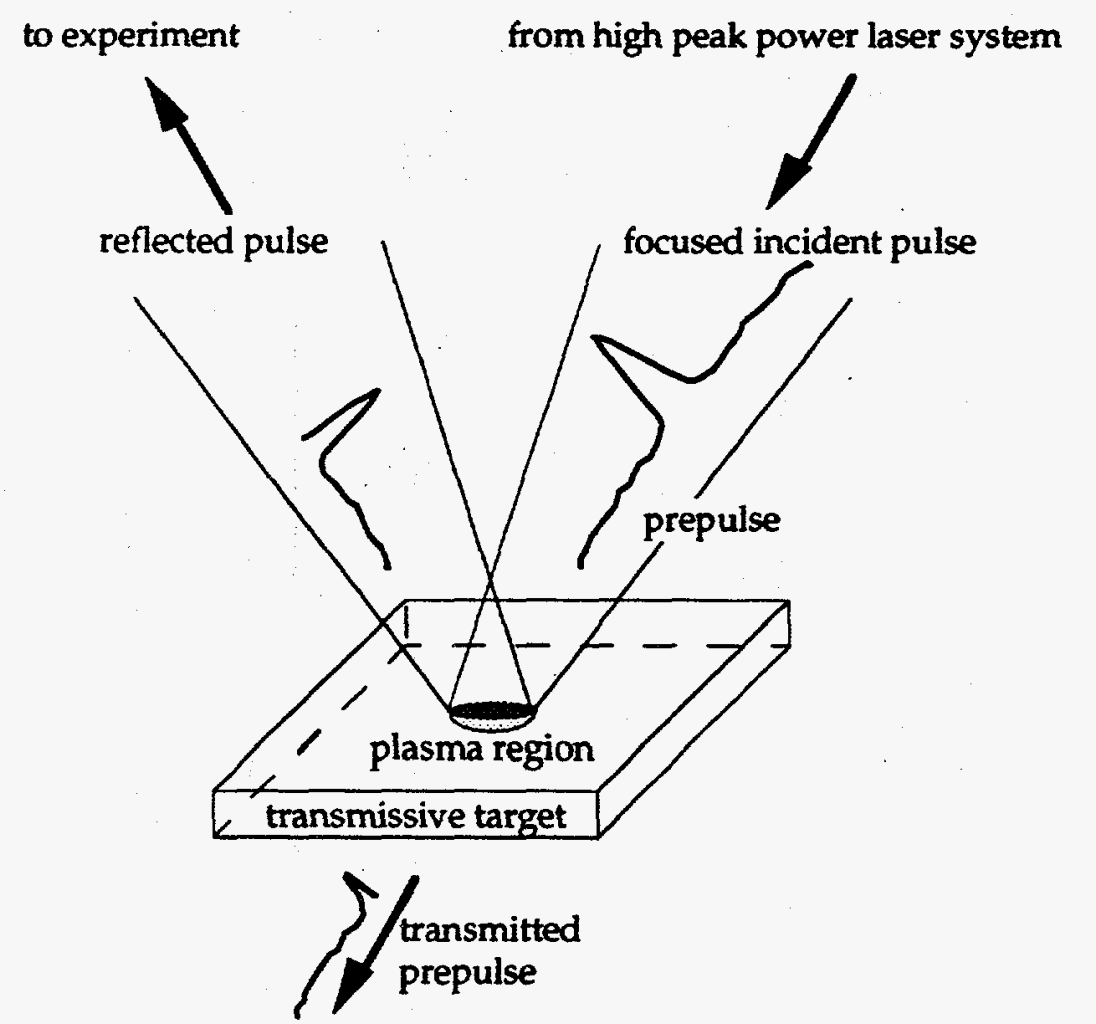

Figure 6.2: The plasma mirror increases the contrast ratio by passing low-intensity prepulse and reflecting the high-intensity, femtosecond pulse.

As a result of their intensity difference, the focused prepulse is mostly transmitted and only weakly reflected with the low target surface reflectivity, while the main pulse is strongly reflected from the dense plasma. We note that 
the incident pulse also contains postpulse energy that may have the same origins as prepulse energy. The plasma formed by the main pulse remains highly reflective for several picoseconds after its formation so this postpulse will be reflected. The picosecond recovery time of these plasmas affects any potential postpulse shaping. Possible schemes to circumvent this plasma recovery time and reduce both prepulse and postpulse energy are proposed in Section 6.7.

The resulting reflected pulse has several desirable characteristics. First, it has a significantly increased contrast ratio between main pulse and prepulse energy and thus is more useful for "clean" experiments involving high-intensity laser interactions with matter. For example, if the prepulse energy reflects at a surface reflectivity of $\sim 5 \%$ and the main pulse reflects off the plasma at $\sim 50 \%$, the reflected light contrast ratio is increased by an order of magnitude. Energy reflectivity measurements presented in Chapter 4 , show the contrast ratio may be improved by at least this factor. Techniques to improve the contrast ratio beyond this factor include: (1) Using an anti-reflective (AR) coating on the target to minimize the prepulse surface reflection. An AR coating typically reduces the low-intensity reflectivity to $0.1 \%$, so the contrast ratio is expected to improve by a factor of 500 at $50 \%$ reflectivity. (2) Placing the target at Brewster's angle, and using P-polarized light to minimize prepulse surface reflection. At the ideal Brewster's angle, for the center wavelength, the reflectivity should be close to zero. A reflectivity measurement at Brewster's angle gave $<0.1 \%$ (at the instrument noise level). Using this figure, the contrast ratio could be improved by a factor of $>500$. Disadvantages of Brewster's angle include a lower on-target intensity (greater area), as well as lower plasma reflectivity at this angle and polarization. (3) Using a series of plasma mirrors to successively improve the contrast ratio. Theoretically, this design could reduce prepulse to acceptable levels regardless of laser intensity. However, finding a convenient geometry for 
multiple plasma mirrors, focusing and alignment difficulties, and overall energy loss may make this system impractical.

It should be noted that Brewster's angle may not be the optimum plasma mirror angle. The problem depends not only on the minimum reflection angle for prepulse, predicted by the Fresnel equations [2], but also on the best angle for main pulse plasma reflection. An obliquely incident, P-polarized field experiences resonance absorption in a plasma. Fractional absorption, $f_{A^{\prime}}$ due to resonance absorption varies with the angle of incidence, $\theta$, as [96]

$$
f_{A}=5.12 \tau^{2} \exp \left(-4 \tau^{3} / 3\right)
$$

where $\tau=(\omega L / c)^{4 / 3} \sin \theta$, and $L$ is the plasma scalelength. On-target intensity also varies as $\cos \theta$, because energy is spread over an elliptical area. The area change affects the prepulse and main pulse equally, so the contrast ratio will not change from a simple area effect. However, the change in intensity nonlinearly changes both the plasma temperature and density and, ultimately, the main pulse reflectivity. Finally, the incident angle affects the optical pathlength (pathlength $x$ optical density) of plasma available for absorption by other mechanisms such as inverse Bremsstrahlung. The competition between all these effects must be accounted for to maximize the contrast ratio and can be expressed as

$$
\text { contrast ratio }=\frac{I_{\text {main pulse }} R[\text { res. abs. }(\theta), \text { area }(\theta), \text { inv. brem. }(\theta)]}{I_{\text {propulse }} R[\text { Fresnel }(\theta)]}
$$

where $R$ is the reflectivity that depends on the various angle-dependent terms of Fresnel reflectivity, resonance absorption, area effects, and inverse Bremsstrahlung. The angle of maximum resonance absorption is calculated [98, $114]$, with a strong dependence on the plasma scale length and collisionality, to appear near Brewster's angle, so is not clear that the best angle for the plasma mirror is Brewster's angle. The best way to determine whether Brewster's angle 
is optimum is probably empirically. Contrast ratio enhancement as a function of angle is discussed in Section 6.5 with respect to liquid plasma mirror results.

A second desirable characteristic of light reflected off the plasma mirror is that the pulse reflection is highly specular, because plasma expansion is small and plasma density profiles are flat. If we assume the plasma expansion velocity, $\mathrm{v}$, is approximately equal the local plasma sound speed, $c_{\mathrm{s}}$,

$$
v=c_{s}=\left(Z T_{e} / M_{i}\right)^{1 / 2} \sim 10^{7} \mathrm{~cm} / \mathrm{s}=1 \AA / f_{s}
$$

the $100 \AA$ of plasma expansion during a $\sim 100$ fs pulsewidth is significantly less than one-quarter wavelength at visible wavelengths. Hydrodynamic expansion is considered to be roughly planar if the scale length $c_{s} \tau_{1}$ is such that [94]

$$
c_{s} \tau_{l}<2 R_{\text {foos spot }}
$$

where $c_{s}$ is the plasma sound speed, $\tau_{l}$ is the laser pulsewidth and $R_{\text {focal spot }}$ is the focal spot radius. For our scale length of $c_{\mathrm{s}} \tau_{l} \sim 100 \AA$ and focal spot $\approx 30 \mu \mathrm{m}$, the geometry is well within planar limits. Data showing the plasma mirror effect on the pulse spatial profile, including the spatial filtering effect, is presented in Section 6.4.2.

Third, the reflected pulsewidth is shorter than the incident pulsewidth because a portion of the main pulse below the target damage threshold transmits along with the prepulse, and a portion of the leading edge creates plasma. The exact point in the main pulse at which critical plasma forms is a complex laser damage issue depending, to varying degrees, on material damage threshold, laser intensity, integrated pulse energy, pulseshape, pulsewidth, and polarization, among other parameters. A simple model in Section 6.6 will demonstrate this pulse shortening effect and data is presented in Section 6.4.3. 


\subsection{WORK RELATED TO THE PLASMA MIRROR}

Reflective plasmas have been applied as energy switches in various systems over several different spectral regions and with a variety of methods used to produce the plasma. A self-induced plasma mirror in the visible region with high-intensity, ultra-short pulses extends these techniques. In this regime, a high-intensity pulse is necessary to induce ionization breakdown and reflective plasma formation within some fraction of the ultra-short incident pulse duration. Solid, liquid or high-density gas targets are also convenient to provide critical electron density to reflect the visible light pulses $\left(>10^{21} \mathrm{~cm}^{-3}\right)$.

In the microwave region, a discharge plasma was used to switch energy stored in a resonator to an external load in a short time, producing high power microwave pulses [191]. Using a $9.2 \mathrm{GHz}$ microwave frequency, a critical electron density of $\sim 10^{12} \mathrm{~cm}^{-3}$ was necessary to produce pulses of $\sim 100 \mathrm{~ns}$.

In the infrared (IR) region, semiconductor switching has been used to generate picosecond pulses at $10 \mu \mathrm{m}$ [182] and ultra-short, 130fs, pulses at $9.5 \mu \mathrm{m}$ [181]. Semiconductor switching is achieved, in the configuration of Rolland and Corkum [181], by illuminating a semiconductor simultaneously with a $\sim 100 \mathrm{ns,}$ $70 \mathrm{~mJ}$ TEA $\mathrm{CO}_{2}$ laser pulse and a $500 \mu \mathrm{J}, 70 \mathrm{fs}$ visible laser pulse. A free-carrier plasma created by the visible light acts to reflect the $\mathbb{R}$ light. A combination of two semiconductor elements allowed an ultra-short IR pulse to be formed. The first semiconductor (CdTe) was a reflection switch, establishing the IR pulse risetime. The second semiconductor (Si) was a transmission switch, cutting off IR light by reflecting it away. The IR pulsewidth can be varied continuously by using an adjustable delay line to change the relative length of the IR and visible beam paths. With IR radiation at $9.5 \mu \mathrm{m}$, a critical density of $\sim 10^{19} \mathrm{~cm}^{-3}$ was necessary for the semiconductor switching to produce pulses as short as $130 \mathrm{fs}$. 
Work on plasma mirror spatial filtering has also been conducted in the IR region $(1.064 \mu \mathrm{m})$ with 30 ps pulses by Moncur [192]. In this work, a solid or high pressure gas target, instead of a pinhole, is placed at the focal position of a lens. A plasma is formed which reflects a portion of the pulse into amplifiers and the high energy beam is used for irradiating pellets in inertial confinement fusion studies. The reflected beam exhibits a smooth time-integrated spatial intensity profile. The smoothing is due to plasma mirror spatial filtering. It is produced in much the same way a pinhole spatially filters a beam. High spatial frequency intensity ripples in the incident beam appear outside the central focal spot where hot, highly reflective plasma is formed. As a result, intensity fluctuations are not strongly reflected and only a smooth spatial intensity profile is reflected. Thus, the plasma mirror acts like a "pin mirror," rather than a pinhole, to smooth the beam profile. Direct measurements of plasma mirror beam smoothing properties investigated in this research are presented in Section 6.4.2.

Based on earlier prepulse suppression developments at this laboratory [193], indirect evidence was obtained for a plasma mirror's feasibility in the visible region. The duration of $\mathrm{x}$-ray yields from solid targets irradiated by an intense, ultra-short pulse laser beam was monitored [194]. The plasma mirror was created by a $<3 \mathrm{~mJ}, 130 \mathrm{fs} \sim 614 \mathrm{~nm}$ laser focused onto a quartz target under vacuum. The reflected pulse was refocused onto a silicon target and x-ray measurements were taken with a streak camera. To observe the effect of ASE prepulse, a pulse containing a $15 \%$ energy fraction in a 5-10ns pedestal could be introduced by taking out a saturable absorber in the laser system which normally had about $0.1 \%$ ASE prepulse energy. The $x$-ray pulsewidth from the silicon target with a plasma mirror introduced and with 15\% ASE prepulse energy was $3.4 \pm 0.3 \mathrm{ps}$. Without the plasma mirror, the $\mathrm{x}$-ray pulsewidth was $3.1 \pm 0.4 \mathrm{ps}$. Finally, with a $15 \%$ energy pedestal and no plasma mirror, the $x$-ray pulsewidth 
increased to $20 \pm 8 \mathrm{ps}$. The streak camera instrumental response was $3 \mathrm{ps}$ Time resolution was slightly degraded because lower signal intensities than previous work by this group [195] required more signal averaging. Thus, the introduction of a relatively large amount (15\%) of prepulse energy dramatically increases the $x$-ray pulsewidth coming off the target. These results gave indirect evidence that a significant prepulse energy (15\%) vaporized the target before the main pulse could interact with it. The main ultra-short pulse was then absorbed in this lower density plasma. The lower density plasma had significantly smaller collisional cooling rates resulting in a much longer pulse of $x$-rays coming off the target [196]. Short, $\sim 3 p s$, pulse of $x$-rays produced in the other prepulse experiments indicated a high-density plasma with rapid collisional cooling. With respect to the short, $\sim 3 p s$, pulse $x$-ray results, the plasma mirror seemed to bring prepulse energy to a level that mimics the low prepulse achieved by the laser with a saturable absorber introduced. The research presented in this work presents the first direct measurement of a reduced prepulse and modifications to the main pulse envelope due to the plasma mirror.

\subsection{THE SOLID PLASMA MIRROR EXPERIMENT}

The experimental setup is illustrated in Figure 6.3. The plasma mirror target was an uncoated, 0.0625 inch thick, fused quartz $\left(\mathrm{SiO}_{2}\right)$ slide placed at $45^{\circ}$ to the incident beam. The target was mounted in an evacuated chamber (105 Torr) on an $x-y$ translation stage for raster scanning past the laser beam. The incident beam was the amplified, femtosecond dye laser described in Section 2.5 producing $2-3 \mathrm{~mJ},-130 \mathrm{fs}$ pulses at $614 \mathrm{~nm}$ with a repetition rate of $10 \mathrm{~Hz}$. Incident pulses were chopped by a shutter at $5 \mathrm{~Hz}$ to separate raster scan damage spots so a fresh target surface was seen on every laser shot. Pulses were focused onto the quartz slide with an $\mathrm{f} / 10$ achromatic lens giving a focal diameter of $30 \mu \mathrm{m}$ and a 
peak intensity of $\sim 1.4 \times 10^{15} \mathrm{~W} / \mathrm{cm}^{2}$. The reflected beam was recollimated by a another $f / 10$ achromatic lens and sent to either a Spiricon LBA-100 beam profiler or a second-order, single-shot autocorrelator. The incident pulse spatial profile and pulsewidth were taken by replacing the quartz slide with a mirror, translating the focusing lens back so the focus is before the mirror, and moving the collimation lens to recollimate the reflected beam. Incident beam energy was varied by inserting neutral density filters and was monitored by splitting off $\sim 4 \%$ of the incident beam with a window. Reflected energy was measured directly out of the chamber after the collimation lens. All energy measurements were made with a Molectron JD-2000 ratiometer with J3-09 energy probes and stored in a Macintosh HfX computer for later analysis.

A series of measurements were made to characterize the energy, spatial characteristics, pulsewidth, and prepulse suppression of the pulse reflected off a plasma mirror. The measurements demonstrated that the reflected pulse has the useful properties of high energy, ultra-short pulsewidth, suppressed prepulse and a smooth spatial profile. 


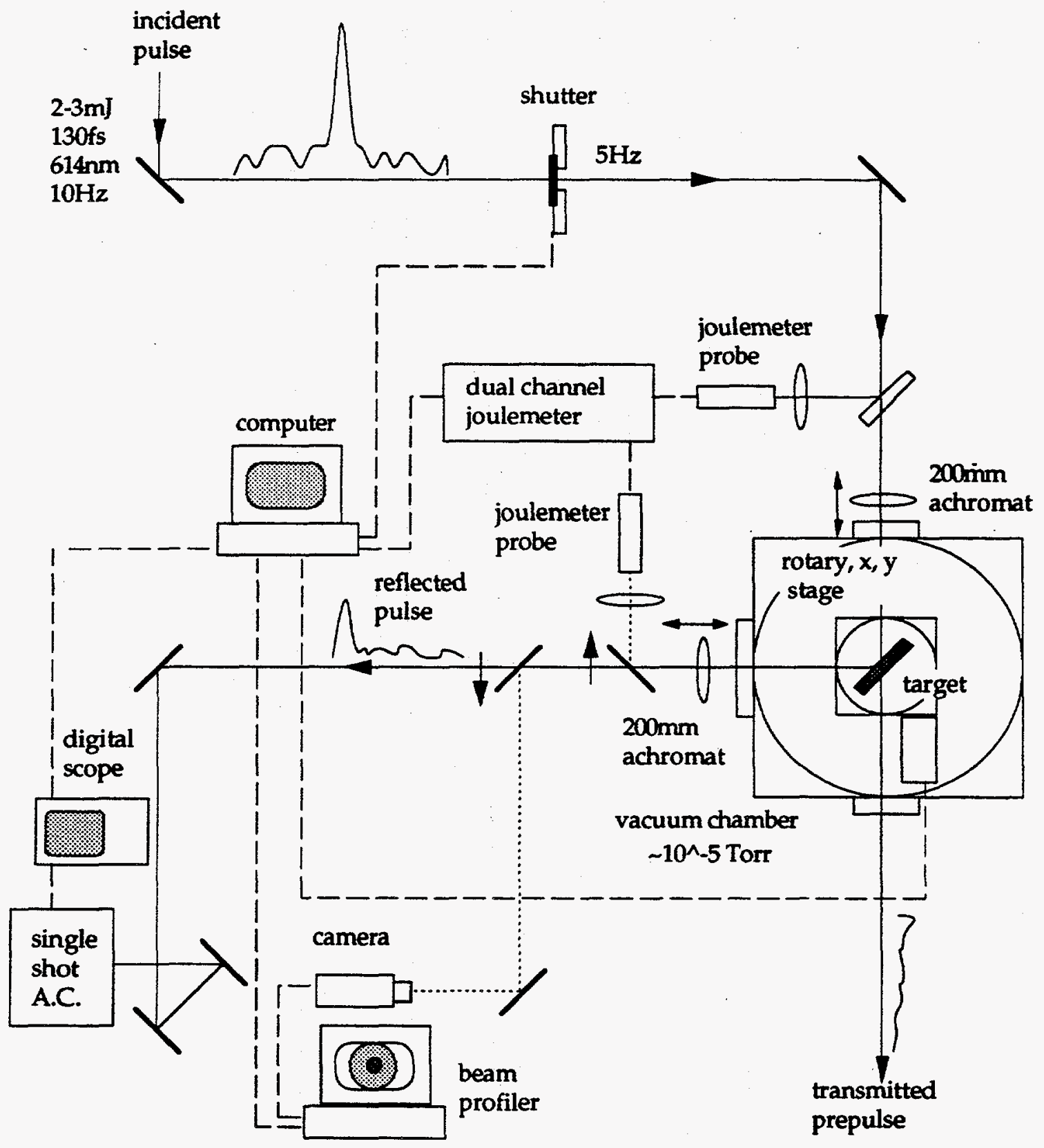

Figure 6.3: Schematic experimental setup for spatial, temporal and reflectioe plasma mirror measurements. 


\subsubsection{Reflectivity measurements}

Plasma energy reflectivity versus incident pulse energy was measured in the following way. Incident energy was varied with neutral density filters and sampled with a calibrated window. Reflected energy was measured directly after the collimation lens. Figure 6.4 shows the characteristic curve of energy reflectivity (10 shot average) versus incident beam energy. The curve displays the salient features of a plasma mirror. Reflectivity at low incident intensity, before any damage occurs, was the cold target surface reflectivity of $\sim 4 \%$. As incident beam energy was increased, reflectivity rose steeply to $\sim 60 \%$ for intensities up to $\sim 2.5 \times 10^{15} \mathrm{~W} / \mathrm{cm}^{2}$. As incident energy was further increased up to $>1.5 \mathrm{~mJ}$, reflectivity asymptotically approached a value of $\sim 70 \%$. Prepulse below the damage threshold was reflected at only $\sim 4 \%$. The intense main pulse caused a nonlinear growth in electron density. Figure 6.4 is plotted on a linear scale to emphasize this nonlinear growth of electron density and reflectivity. The plasma formed acted as an active mirror, nonlinearly changing from $\sim 4 \%$ reflectivity to nearly $70 \%$ within a small fraction of the main pulsewidth.

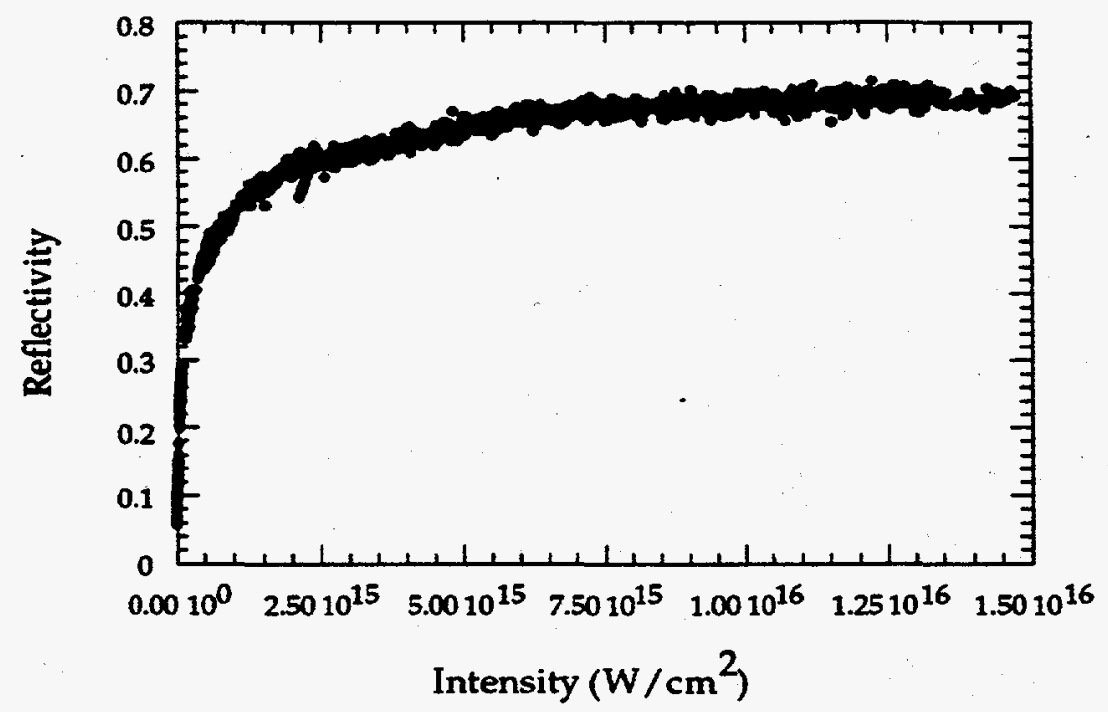

Figure 6.4: Plasma mirror reflectivity versus intensity using uncoated $\mathrm{SiO}_{2}$. 


\subsubsection{Spatial Measurements}

The reflected pulse spatial profile indicated both specular reflection and reflective spatial filtering at high incident intensities. Four representative reflected beam intensity profiles are shown (Figures 6.5 (a)-(d)) in order of increasing incident intensity. Figure 6.5 (a) is a reference picture showing the incident beam profile was approximately flat-top with diffraction rings that arose because the final Bethune dye cell amplifier was overfilled. The beam was unfocused and at low energy, so no plasma mirror was formed. Note that the profile lineout is somewhat skewed to the left because of the reflected intensity off the back target surface, which is easily seen in the associated transverse profile picture.

Figure 6.5 (b) shows the reflected beam profile at full incident intensity of $\sim 1.4 \times 10^{15} \mathrm{~W} / \mathrm{cm}^{2}$. A fit to the profile lineout showed that the recollimated beam became approximately Gaussian. Note that because the incident beam was approximately a flat-top, the focussed beam was nearly an Airy pattern, which is close to a Gaussian. However, we measure the recollimated light, which in a perfect 1-to-1 imaging system should, recollimate as a flat-top again. Clearly, the reflected near-Gaussian profile was not the result of focussing, rather it was induced by the plasma mirror. Most reflected intensity was found in the central region where the incident focussed Airy pattern was most intense and the plasma was hottest and thus most reflective. Note also that diffraction rings present on the incident beam were eliminated. This smoothing was due to plasma mirror spatial filtering [192], and was produced in much the same way a pinhole spatially filters a beam. High spatial frequency intensity ripples in the incident beam appeared outside the central focal spot where hot, highly reflective plasma was formed. As a result, these intensity fluctuations were not strongly reflected and a smooth spatial intensity profile was reflected. Thus, the plasma 
mirror acts more like a "pin mirror" rather than a pinhole to smooth the beam profile.

Some additional points mentioned by Moncur [192] about the spatial characteristics of this beam are worth discussion. Spatial smoothing could be the result of time-averaging during measurements which are insensitive to rapid plasma formation that may produce rapidly varying spatial intensities. If this were true, damage, self-focusing, filamentation, etc., initiated by small intensity fluctuations in the seed beam could still occur during amplification, but they did not [192]. Also, the plasma mirror reduced beam spatial coherence as measured with a wavefront-shearing interferometer [192]. This would at least partially offset intensity fluctuations not detected by time-integrated measurements. Diffraction patterns, produced in an optical path with defects and inhomogeneities, would be suppressed by reduced spatial coherence. Of course, reduced spatial coherence may also degrade reflected beam focusability. However, indirect evidence from $\mathrm{x}$-ray yield measurements showed pedestalsuppressed pulse focusability was not significantly degraded [193]. Direct focusability measurements were not made here, however autocorrelations made without signal degradation indicated the reflected beam focusing was comparable to the incident beam.

Figure 6.5 (c) shows the reflected beam profile at $\sim 2.8 \times 10^{14} \mathrm{~W} / \mathrm{cm}^{2}$. The beam was still round and specular by eye, but the intensity profile showed broader features.

Figure 6.5 (d) shows the reflected beam profile at $\sim 3.5 \times 10^{13} \mathrm{~W} / \mathrm{cm}^{2}$. The electron density was now estimated to be subcritical and a useful plasma mirror was no longer formed. Reflections began to return to low intensity surface reflections that maintain the incident beam spatial profile. Diffraction rings 

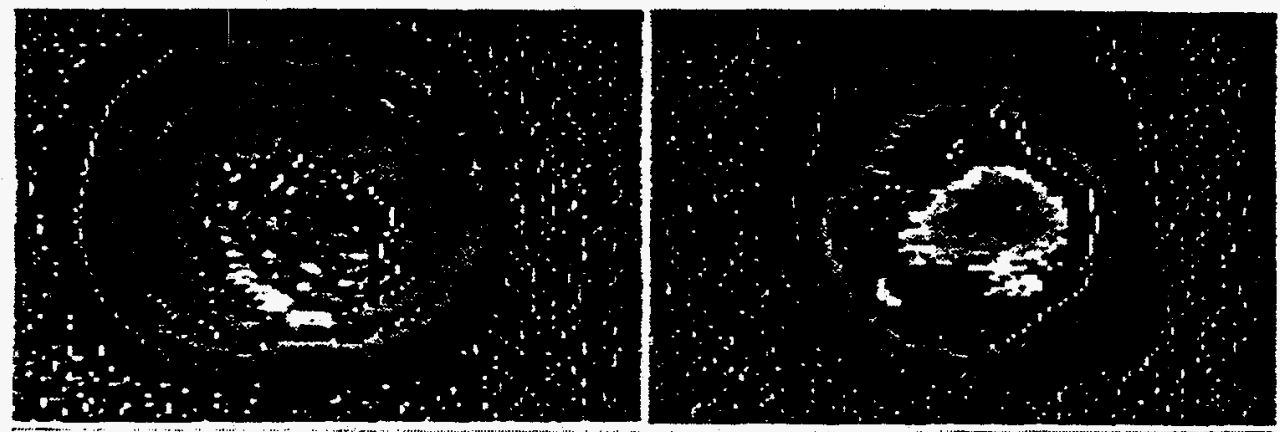

(a)

(b)

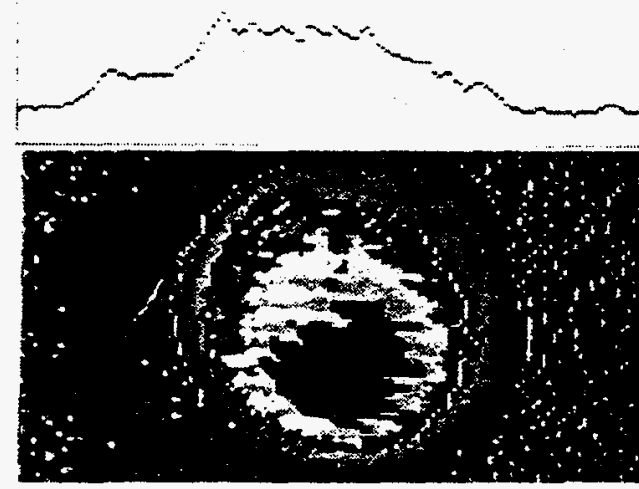

(c)

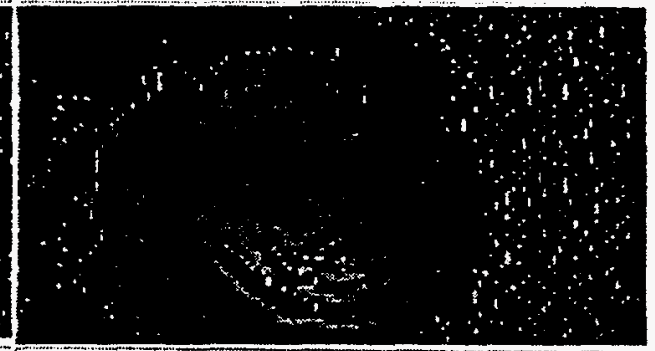

(d)

Figure 6.5: (a) Incident profile and reflected pulse profile at (b) $1.4 x 10^{15} \mathrm{~W} / \mathrm{cm}^{2}$ (c) $2.8 \times 10^{14} \mathrm{~W} / \mathrm{cm}^{2}(d) 3.5 \times 10^{13} \mathrm{~W} / \mathrm{cm}^{2}$

present on the incident beam and the reflection from the back target surface again began to appear.

\subsubsection{Temporal Measurements}

Incident and reflected pulsewidths were measured with a single shot, second order autocorrelator [93]. The reflected pulsewidth was consistently shorter than the incident pulsewidth. In a representative measurement at full intensity, the incident pulse was $140 \mathrm{fs}$ fit to a $\operatorname{sech}^{2} x$ pulseshape. The reflected pulse was $130 \mathrm{fs}$. This $7 \%$ difference in pulsewidth could not be accounted for by shot-to-shot fluctuations in the laser pulsewidth which were measured to be less than $1 \%$. Furthermore, at lower incident intensities, or with materials with a 
higher damage threshold, a greater fraction of the main pulse will leak through and the reflected pulse will be clipped later. Thus the change in the pulsewidth between the incident and reflected pulse is expected to become greater with lower incident energies. This effect is modeled in Section 6.6. In view of this, a complete temporal plasma mirror characterization on the reflected pulse may reveal its use as a "temporal beamsplitter" with incident pulse energy controlling the reflected pulsewidth.

It is important to note that measurements were taken using a single shot, second order autocorrelator discussed in Section 2.6. The signal is proportional to the second order autocorrelation function $G_{2}(\tau)$ which is symmetric in $\tau$ so pulse shape asymmetry does not show up. A single shot, third order autocorrelator is capable of detecting asymmetry in the incident or reflected pulse. The third order autocorrelator measures a signal proportional to the third order correlation function $G_{3}(\tau)$ which is not symmetric in $\tau$ :

$$
G_{3}(\tau) \propto \int_{-\infty}^{\infty} I(t) I^{2}(t-\tau) d t
$$

Determining asymmetry in the incident and reflected pulse is of interest, because it can indicate when the plasma was formed during the incident pulse envelope. The reflected pulse risetime detected by third order autocorrelation gives some insight into the relative plasma formation rate for different plasma mirror experiments as a function of incident intensity. A third-order autocorrelator was constructed for this research, but the results proved unpredictable and did not give any satisfactory indication of pulse asymmetry changes in a consistent manner. 


\subsubsection{Prepulse Suppression Measurements}

An initial test showed the plasma mirror prepulse suppression capability was at least equivalent to a solid saturable absorbing filter (Schott glass RG-630). The advantage of the plasma mirror, of course, is that it acts on a tunneling (femtosecond) timescale while saturable absorbers generally act on an electronic (picosecond) timescale. To induce a large prepulse without changing the laser focusing, the laser timing was changed by nanoseconds, Figure 6.6 (a), until a prepulse large enough to be visible by the diode was present while the laser energy dropped to $\sim 2 \mathrm{~mJ}$. A fast photodiode was used to measure the pulse reflected off the plasma mirror with three different laser configurations. First, the unfocused, high-prepulse laser beam was reflected off an aluminum coated quartz target as a reference signal showing the main pulse with a significant prepulse pedestal (Figure 6.6 (b)). An RG-630 saturable absorber was placed in the laser system to reduce laser prepulse below the diode sensitivity. The measurement was repeated using the unfocused pulse and the aluminum coated target. Only the main pulse signal appeared and the prepulse signal was undetectable (Figure 6.6 (c)). Finally, the RG-630 saturable absorber was removed to return the laser to a high ASE condition, and the laser was focused on an uncoated quartz target. A plasma mirror was formed by the focused pulse and the reflected signal showed no evidence of a prepulse (Figure $6.6(d)$ ). We can see from this simple experiment how the plasma mirror acts as a saturable absorber on an ultra-fast timescale. 

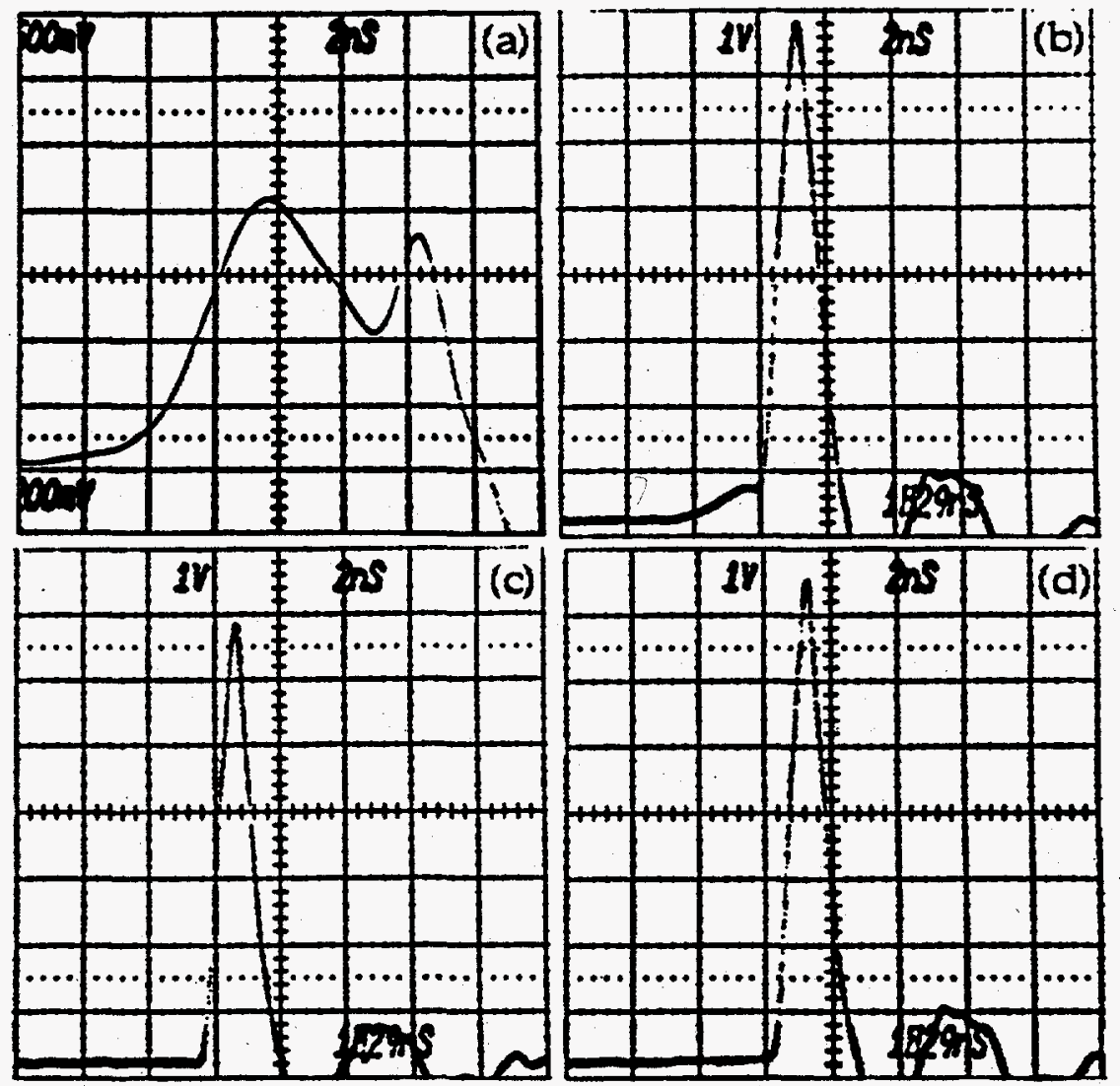

Figure 6.6: (a) Timing between the oscillator (narrow peak) and amplifier (wide peak) pulses (b) Induced prepulse pedestal and main pulse (c) Pulse with RG-630 saturable absorber in laser system (d) Main pulse with no saturable absorber, but reflected off the plasma mirror.

The ultimate test of the plasma mirror capability is to measure the reflected pulse with a high dynamic range detector. To apply the plasma mirror to ultra-high intensity laser systems, we must be able to see about $10^{4}$ improvement in the contrast ratio to insure prepulse below $10^{12} \mathrm{~W} / \mathrm{cm}^{2}$ for a $10^{18} \mathrm{~W} / \mathrm{cm}^{2}$ pulse with an initial contrast ratio of $10^{3}$.

The high-dynamic-range prepulse measurement scheme is illustrated in Figure 6.7. The laser pulse was sent through the slow scanning autocorrelator described in Chapter 2 and signal was detected with a photomultiplier tube (pmt) cooled by a $50 / 50$ mixture of ethylene glycol and water to about $-8^{\circ} \mathrm{C}$. The 
noise level was measured to be sufficient to obtain a signal from the weak low intensity prepulse autocorrelation. As the autocorrelator was scanned to the main pulse, the beam was attenuated by a half-wave plate and polarizer so the signal did not saturate either the pmt, signal amplifier, or doubling efficiency in the KDP crystal. Energy was constantly monitored throughout the autocorrelation scan as a normalization measurement. Amplifier gain was adjusted during the scan and changes were recorded for signal normalization. The final signal was therefore a mosaic of all signals obtained at different attenuations and different amplifier gain settings normalized by these factors.

First, the laser autocorrelation was taken without any plasma mirror (Figure 6.8). The contrast ratio was measured to be $\sim 10^{4}$. Note that the peaks at $\sim 1.5 p$ due to retroreflections from the autocorrelator pellicle, as mentioned in Section 2.5.5.3, appear again. This figure was in good agreement with a simple in situ measurement commonly taken where the seeded $(\sim 3 \mathrm{~m} J)$ to unseeded energy $(<1 \mu \mathrm{J})$ ratio was calculated as an approximate contrast ratio figure $\left(>3 \times 10^{3}\right)$. Following this measurement, a P-polarized beam was focused onto the $\mathrm{SiO}_{2}$ target placed at Brewster's angle $\left(55^{\circ}\right)$ and the measurement was repeated by sending the beam reflected off the plasma mirror to the autocorrelator. Figure 6.8 shows contrast ratio was improved by a factor $>625$. Measurement of contrast improvement was limited by the cooled pmt detector noise level. The measurement was comparable with the suppression predicted in Section 6.2 of $>500$ using $>50 \%$ main pulse reflectivity and $\sim 0.1 \%$ prepulse reflectivity, at Brewster's angle, from the cold target. 


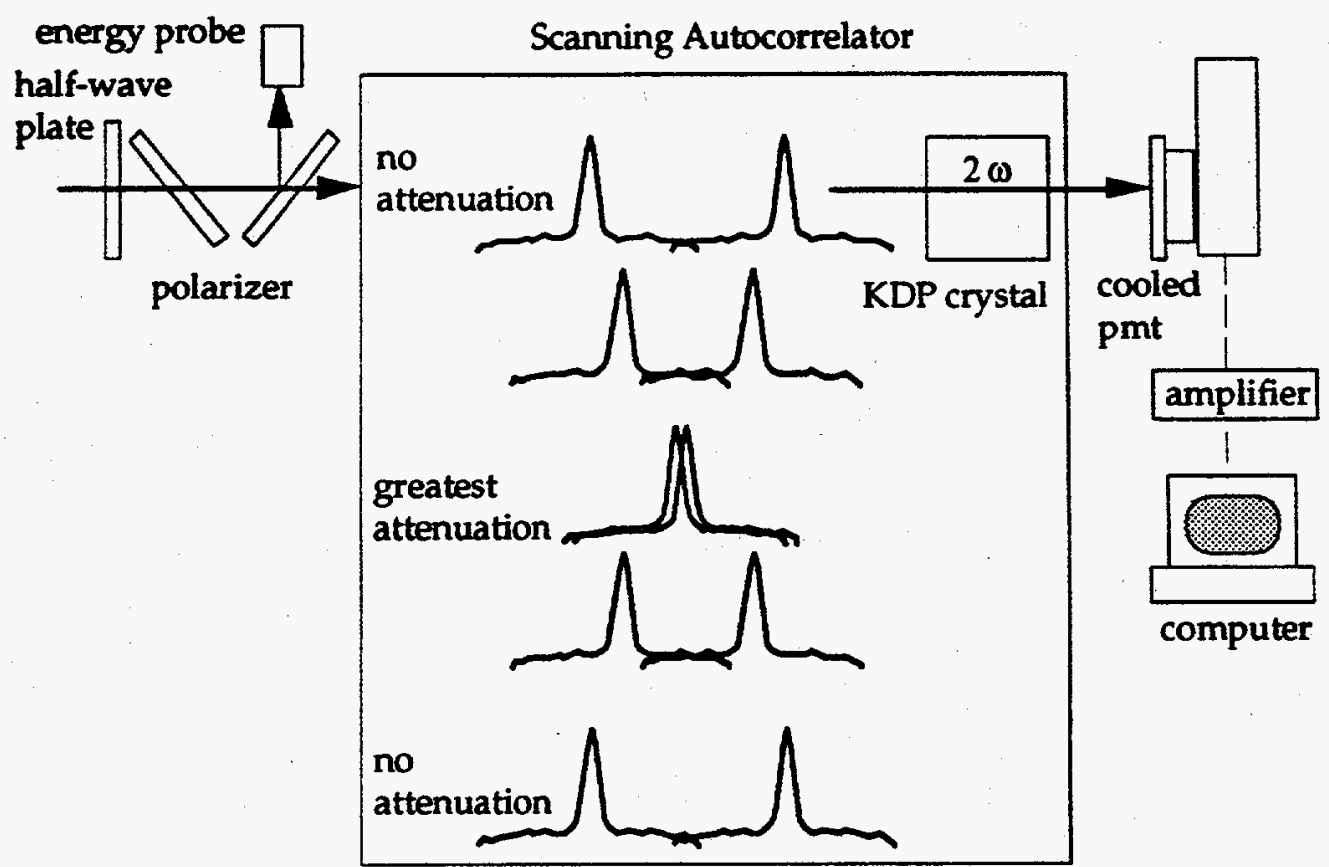

Figure 6.7: Schematic experimental setup for a high-dynamic-range autocorrelation measurement.

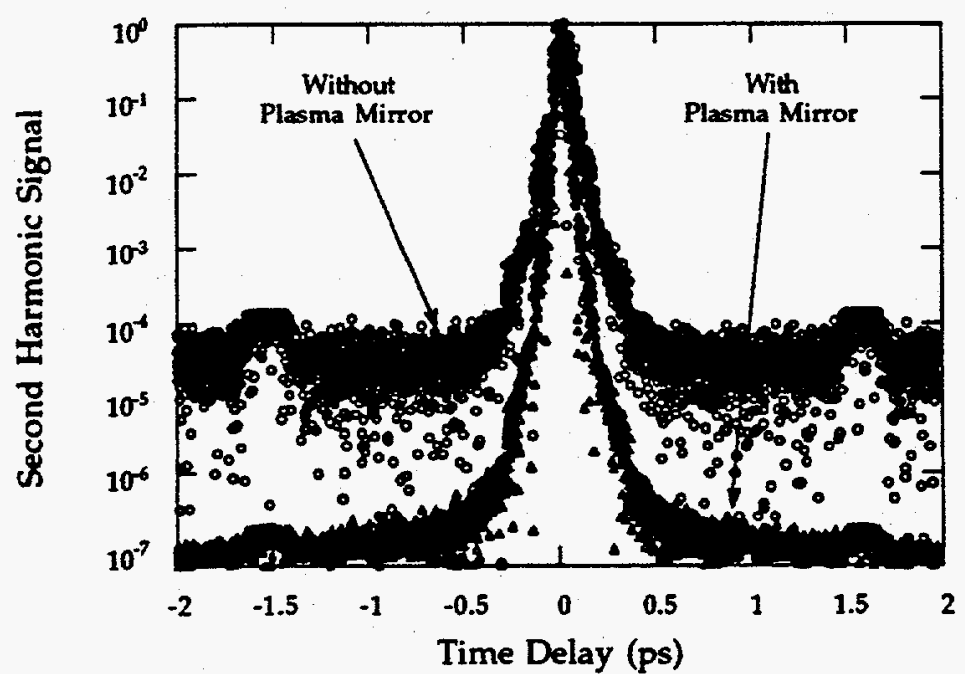

Figure 6.8: High-dynamic-range auto-correlation shows the plasma mirror prepulse suppression at Brewster's angle improves the contrast ratio by a factor $>625$. 


\subsection{THE LIQUID PLASMA MIRROR EXPERIMENT}

A plasma mirror using a liquid jet target extends plasma mirror use to high-repetition-rate, high-intensity laser systems [197] because the liquid target is rapidly self-healing. This method also circumvents the complication of raster scanning solid targets to obtain a fresh surface for each laser shot. A liquid plasma mirror was demonstrated [198] in this laboratory by using a jet of ethylene glycol in vacuum to create a plasma mirror with $\sim 500 \mu$ s recovery time allowing the plasma mirror to be used for lasers operating at up to $2 \mathrm{kHz}$.

The liquid plasma mirror apparatus is shown in Figure 6.9. A sapphire dye-laser jet nozzle is mounted inside a small vacuum chamber and attached to the side on an optical table. Ethylene glycol is pumped through the jet by a small mechanical pump isolated from the vacuum chamber by a pulsation damper. Ethylene glycol has a vapor pressure of $<60 \mathrm{mTorr}$ at $20^{\circ} \mathrm{C}$, so it will not boil off in the vacuum chamber at a base pressure $>60 \mathrm{~m}$ Torr.

The chamber has entry and exit windows placed to allow reflection from the jet at Brewster's angle. The chamber bottom serves as the fluid reservoir which gravity feeds the pump located below. A water-filled heat exchanger is used to cool the ethylene glycol below $15^{\circ} \mathrm{C}$, increasing the jet's laminar flow stability and preventing fluid condensation on the chamber windows. 


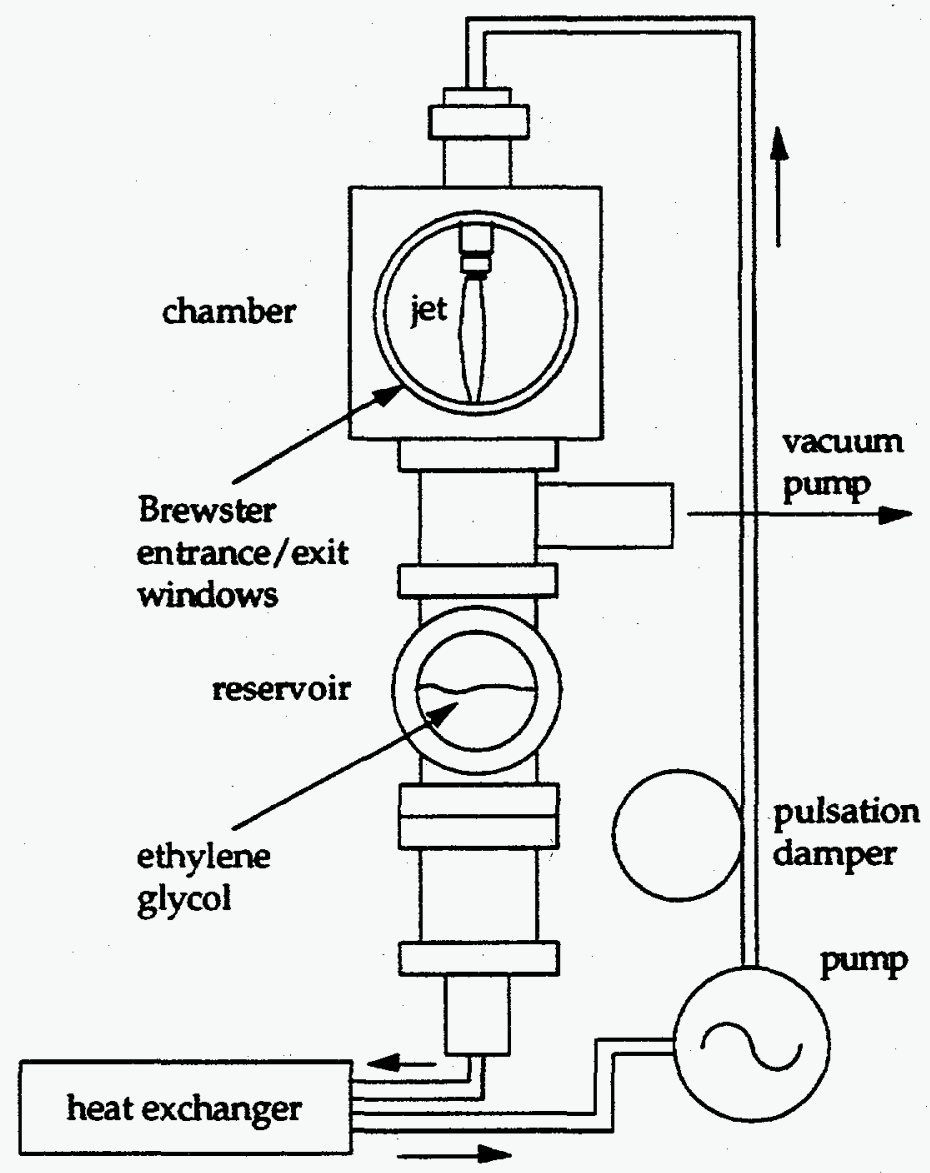

Figure 6.9: Schematic diagram of the liquid plasma mirror.

Initial jet dynamics tests were performed by Backus [198] using a focused $200 \mathrm{~mJ}, \sim 7 \mathrm{~ns} \mathrm{Nd}$ Y $\mathrm{AG}$ laser pulse to create a plasma and a He-Ne laser probe. The jet response showed two periods when the He-Ne beam reflection was disturbed. There is an initial drop in reflectivity for $\sim 150 \mu$ s due to the formation of an absorptive long pulse ( $\mathrm{ns})$ plasma from the Nd:YAG laser. The reflected intensity recovers to its initial level until $\sim 250 \mu$ s when a second smaller drop in the jet reflectivity is observed. By varying the focal position in the jet, it was determined that the second reflectivity drop is due to an acoustic wave that travels up the jet at $\sim 13 \mathrm{~m} / \mathrm{s}$ and reflects from the nozzle face. Full jet recovery was seen in $\sim 500 \mu \mathrm{s}$. 
Prepulse suppression capability for ultra-short pulses was tested using diodes in an experiment similar to the solid plasma mirror experiment described in Section 6.4.4. Ultra-short pulses were produced by the amplified dye laser described in Section 2.5. The beam was focused onto the jet at Brewster's angle and the incident and reflected pulses were monitored with a vacuum photodiode and a Tektronix 7104 oscilloscope. Figure 6.10 (a) shows the incident pulse with an artificially high ASE prepulse clearly visible. High ( $12 \%$ in energy) ASE prepulse was introduced by removing a RG640 saturable absorber and the first spatial filter. The signal from the pulse reflected off the plasma mirror is shown overlaid on the incident pulse signal. It shows no evidence of a prepulse within the diode-oscilloscope sensitivity limits and has a $1 \mathrm{GHz}$ oscilloscope bandwidthlimited risetime. Note that the structure behind the pulse is electronic ringing noise. Figure 6.10 (b) shows the same measurement, but with a lower prepulse level after an RG-630 saturable absorber filter was placed in the laser. The prepulse level still was reduced to a level below that of a saturable absorber, demonstrating the plasma mirror effectiveness over conventional saturable absorber methods.

Reflectivity results as a function of the lens focal length, angle of incidence, and incident pulse energy are shown in Table 6.1. Two Molectron J309 energy probes monitored the shot-to-shot reflectivity. A polarizer and halfwave plate were used to adjust the beam energy. ASE prepulse energy was measured to be $<0.1 \%$ of the total pulse energy by blocking the oscillator seed beam. Breakdown in the jet occurred with about $300 \mu \mathrm{J}$ of focused ASE energy (100mm lens, Brewster's angle), far above our highest ASE level for the data taken. 


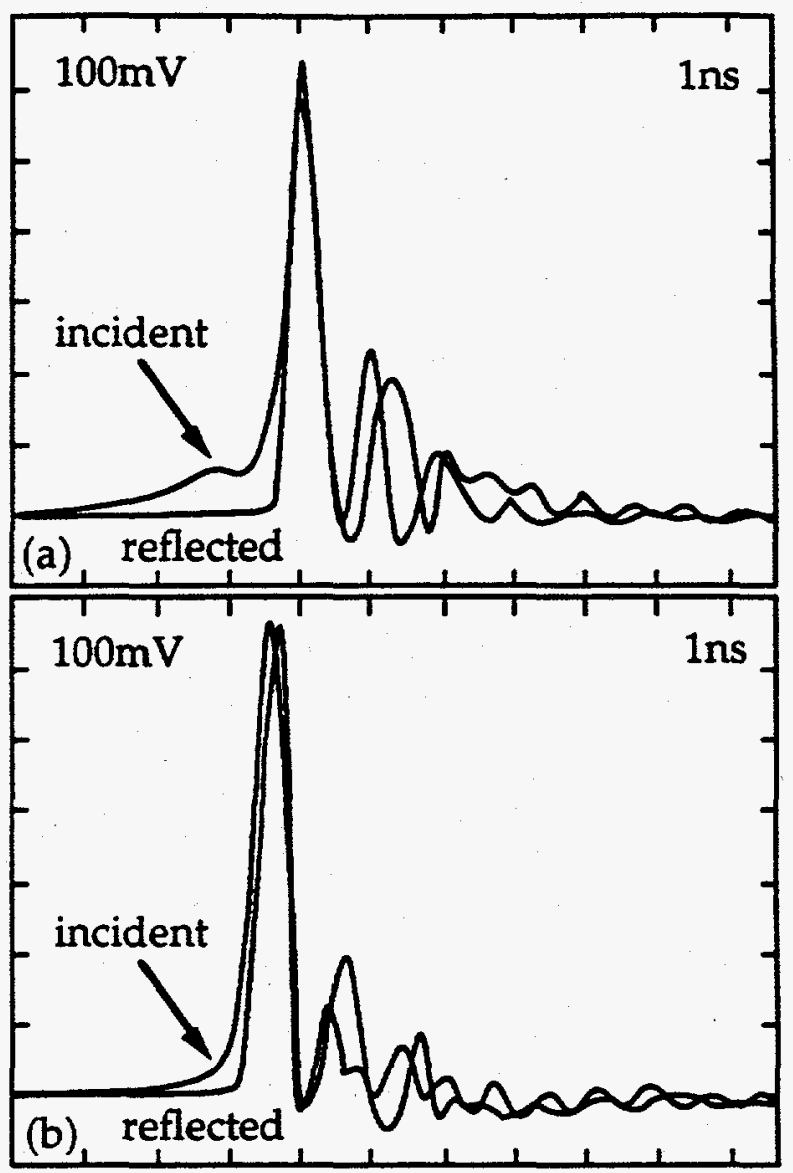

Figure 6.10: Liquid plasma mirror prepulse reduction (a) with a $12 \%$ prepulse energy introduced (b) with a $12 \%$ prepulse energy and an RG-630 saturable absorber. Note that the plasma mirror is effective in both cases.

Reflectivity data were consistent with observed values from solid quartz targets. Reflectivity as a function of intensity also had the same intensitydependent features as solid target data. The most significant difference was that the reflectivity at high angles of incidence is significantly lower than expected. A simple Drude model Fresnel reflectivity estimate of a $50 \mathrm{eV}$ plasma gives values of $75 \%$ at normal incidence and $55 \%$ at Brewster's angle. 


\begin{tabular}{ccccccc}
\hline $\begin{array}{c}\text { Angle } \\
\text { (degrees) }\end{array}$ & $\begin{array}{c}\text { Focal Length } \\
(\mathbf{m m})\end{array}$ & $\begin{array}{c}\text { Intensity } \\
\left(\mathbf{1 0}^{\mathbf{1 6}} \mathbf{W} / \mathrm{cm}^{2}\right)\end{array}$ & Polarization & $\begin{array}{c}\text { Measured } \\
\text { Reflectivity } \\
(\%)\end{array}$ & $\begin{array}{c}\text { Predicted } \\
\text { Reflectivity } \\
(\%)\end{array}$ & $\begin{array}{c}\text { Calculated } \\
\text { Contrast } \\
\text { Enhancement }\end{array}$ \\
\hline 0 & 200 & 2.2 & & 60 & 60 & 19 \\
0 & 160 & 3.4 & & 65 & 65 & 21 \\
0 & 100 & 8.6 & & 70 & 70 & 23 \\
45 & 200 & 1.5 & $\mathrm{~S} / \mathrm{P}$ & $45 / 28$ & NC & $6 / 49$ \\
45 & 160 & 2.4 & $\mathrm{~S} / \mathrm{P}$ & $55 / 30$ & $N C$ & $7 / 53$ \\
45 & 100 & 6.1 & $\mathrm{~S} / \mathrm{P}$ & $80 / 50$ & $N C$ & $11 / 88$ \\
55 & 200 & 1.2 & $\mathrm{~S} / \mathrm{P}$ & $50 / 22$ & $N C / 40$ & $4 / 220$ \\
55 & 160 & 1.9 & $\mathrm{P}$ & 24 & 50 & 240 \\
55 & 100 & 5.0 & $\mathrm{P}$ & 38 & 54 & 380 \\
\hline
\end{tabular}

Table 6.1: Observed reflectivity and calculated contrast enhancement factor for a 2.5mJ, 140fs pulse as a function of angle of incidence, focal spot and polarization [198]. Intensities are the peak intensity assuming a focal spot 1.5 times diffraction limit. Contrast ratio enhancements assume a prepulse reflectioity equal to the cold surface reflectivity of ethylene glycol. Italicized numbers are calculated and others are measured directly. Predicted reflectivity results are from the numerical heat diffusion model mentioned below. NC indicates no calculation was done at this angle.

In an attempt to explain this discrepancy, a numerical heat diffusion model $[6,199]$ was used by Backus [198]. Surface reflectivity was modeled as a function of time by using a Drude model and the Fresnel equations, with the electron density given by the simple LTE form, the Saha equation. The initial electrons generated by MPI were simulated by using a momentary density of $\sim 1$ electron per ethylene glycol molecule, introduced at a threshold intensity of $10^{15} \mathrm{~W} / \mathrm{cm}^{2}$. This initial ad hoc guess at the electron density is justified, because results are relatively insensitive to the initial, nonzero, electron density. To compare with the data, the peak simulation intensity $\left(1016 \mathrm{~W} / \mathrm{cm}^{2}\right)$ was chosen to match the highest observed reflectivity ( $70 \%$ for normal incidence using a $100 \mathrm{~mm}$ 
lens). Other intensities were scaled according to their expected spot size. Results predict reflectivities of $70 \%, 65 \%$, and $60 \%$ for normal incidence with the 100,160 , and $200 \mathrm{~mm}$ lenses respectively (Table 6.1). Normal incidence values are in good agreement with this experiment, but the Brewster's angle calculations consistently predict a reflectivity above the measured value. The most likely cause for the discrepancy is the presence of a small plasma scale length. The model did not include effects from any hydrodynamic expansion and assumed only the Fresnel reflectivities of a sharp interface. Results at a high angle of incidence are more sensitive to the presence of even a small scale length than are normal incidence results [167].

The spatial effects on the reflected beam were very similar to those observed in the solid plasma mirror experiments. The spatial filtering and plasma smoothing effects were readily seen, and focusability was not affected. The jet was run in vacuum, in a cold, laminar flow, so it remained optically flat. Variations in surface flatness therefore were no greater than the solid targets, so there is no effect on focusability from the liquid-vacuum interface.

Pulsewidth measurements indicate a pulse shortening similar to that found in the solid target experiments. The pulsewidth was reduced by more than $10 \%$, from $141 \mathrm{fs}$ to $125 \mathrm{fs}$, upon reflection.

\subsection{A MODEL FOR THE PLASMA MIRROR}

A simple model was developed to study the effect of rapid reflective plasma mirror switching action on the reflected pulse. The model assumed only Fresnel reflectivity and a plasma generated mainly by MPI. The MPI form was used for the ionization process because it was easy to implement in the simulation and did not significantly alter the physics. Results clearly indicate how the femtosecond rise in the reflectivity virtually splits the pulse temporally 
when the plasma density grows beyond critical density. The point at which the incident pulse splits is determined solely by the incident intensity and laser pulseshape for a given target.

The model begins with the simple Fresnel formula for P-polarized light.

$$
R_{p}=\left|\frac{\varepsilon \cos \theta-\sqrt{\varepsilon-\sin ^{2} \theta}}{\varepsilon \cos \theta+\sqrt{\varepsilon-\sin ^{2} \theta}}\right|^{2}
$$

where $\varepsilon$ is the dielectric constant and $\theta$ is the laser beam angle of incidence. The dielectric constant is composed of a solid, or bound electrons, part, $\varepsilon_{0}$, and a plasma, or free electrons, part, $\omega_{p}{ }^{2} / \omega^{2}$, where $\omega_{p}$ is the plasma frequency and $\omega$ is the laser frequency.

$$
\varepsilon=\varepsilon_{0}-\frac{\omega_{p}^{2}}{\omega^{2}}
$$

Electron production depends mainly on the MPI ionization rate so

$$
\frac{d n_{e}}{d t} \sim \sigma_{N} I^{N}
$$

but a more exact form is used to include impact ionization, radiative, dielectronic, and 3-body recombination, all with an effective rate

$$
\frac{d n_{e}}{d t}=\eta \sigma_{N} I^{N}+\frac{n_{e}}{\tau_{a}}-\frac{n_{e}^{2}}{\tau_{r}}
$$

where $\eta$ is a proportionality constant, and $\tau_{a}$ and $\tau_{r}$ are the effective time constants for avalanche and recombination processes.

The incident pulse is given a 100fs FWHM, sech 2 temporal profile with a

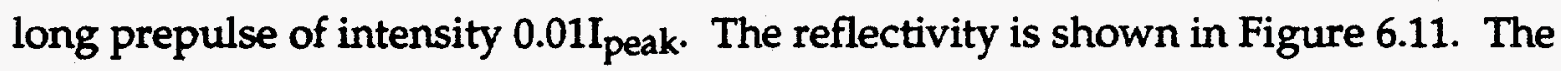
input pulse for this plot has a peak intensity of $2.5 \times 10^{15} \mathrm{~W} / \mathrm{cm}^{2}$. A five-photon cross section, $\sigma_{5}=1.05 \times 10^{-157} \mathrm{~cm}^{10} s^{4}$, is assumed because the ionization potential is $\sim 9 \mathrm{eV}$ and the photon energy at $614 \mathrm{~nm}$ is $\sim 2 \mathrm{eV}$. The avalanche and recombination rates are set at $10^{12} \mathrm{~s}^{-1}$ and $10^{11} / 2 \times 10^{22} \mathrm{~s}^{-1} \mathrm{~cm}^{-3}$, respectively, where $2 \times 10^{22}$ is the density of our quartz sample. 


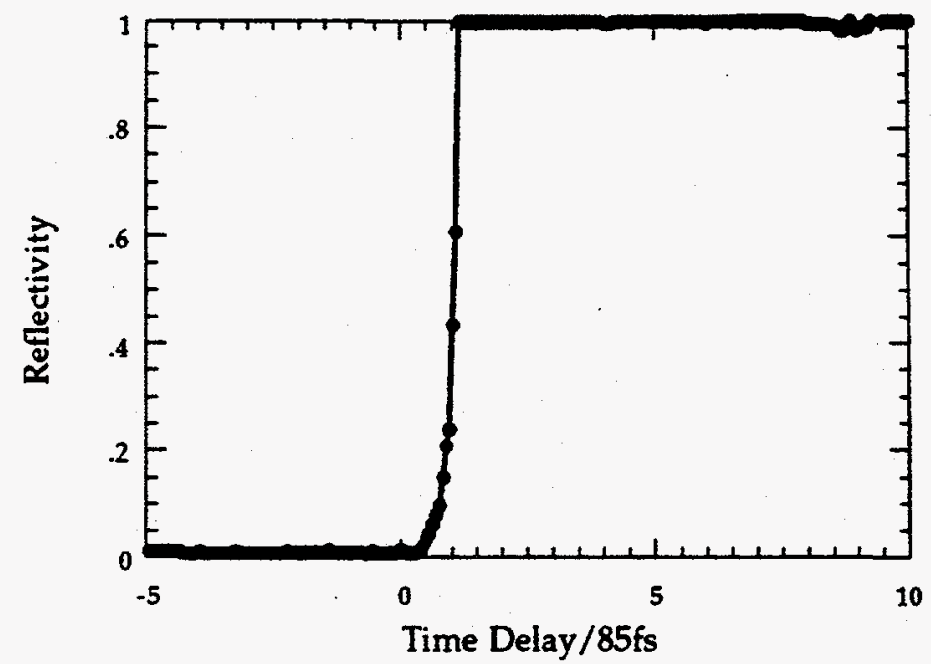

Figure 6.11: Calculated reflection coefficients versus time for a 100fs pulse with intensity $2.5 \times 10^{15} \mathrm{~W} / \mathrm{cm}^{2}$.

The reflectivity rises extremely rapidly acting to slice the pulse temporally virtually at a single point. Plasma reflectivity risetime acts in a nearly binary, "shutter-like" fashion. If the risetime were significantly longer, the reflecting plasma would affect the entire incident pulse. This is an important point for using the reflected pulse in a high-density plasma experiment. The critical factors for these experiments are a large amount of energy available for absorption and a steeply rising leading edge to deposit the energy quickly before hydrodynamic expansion begins. Since the reflectivity risetime is so short and appears in the leading edge, it both steepens the rising edge and preserves the majority of the pulse energy.

The reflectivity obtained can then be multiplied with the incident pulseshape to show the predicted effect of plasma formation on the reflected pulseshape. The products of reflectivity curves generated from incident pulses, $150 \mathrm{fs}$, with peak intensities from $1.0 \times 10^{14} \mathrm{~W} / \mathrm{cm}^{2}$ to $10^{16} \mathrm{~W} / \mathrm{cm}^{2}$ with the incident pulse at these peak intensities are shown in Figures 6.12 (a)-(d). 

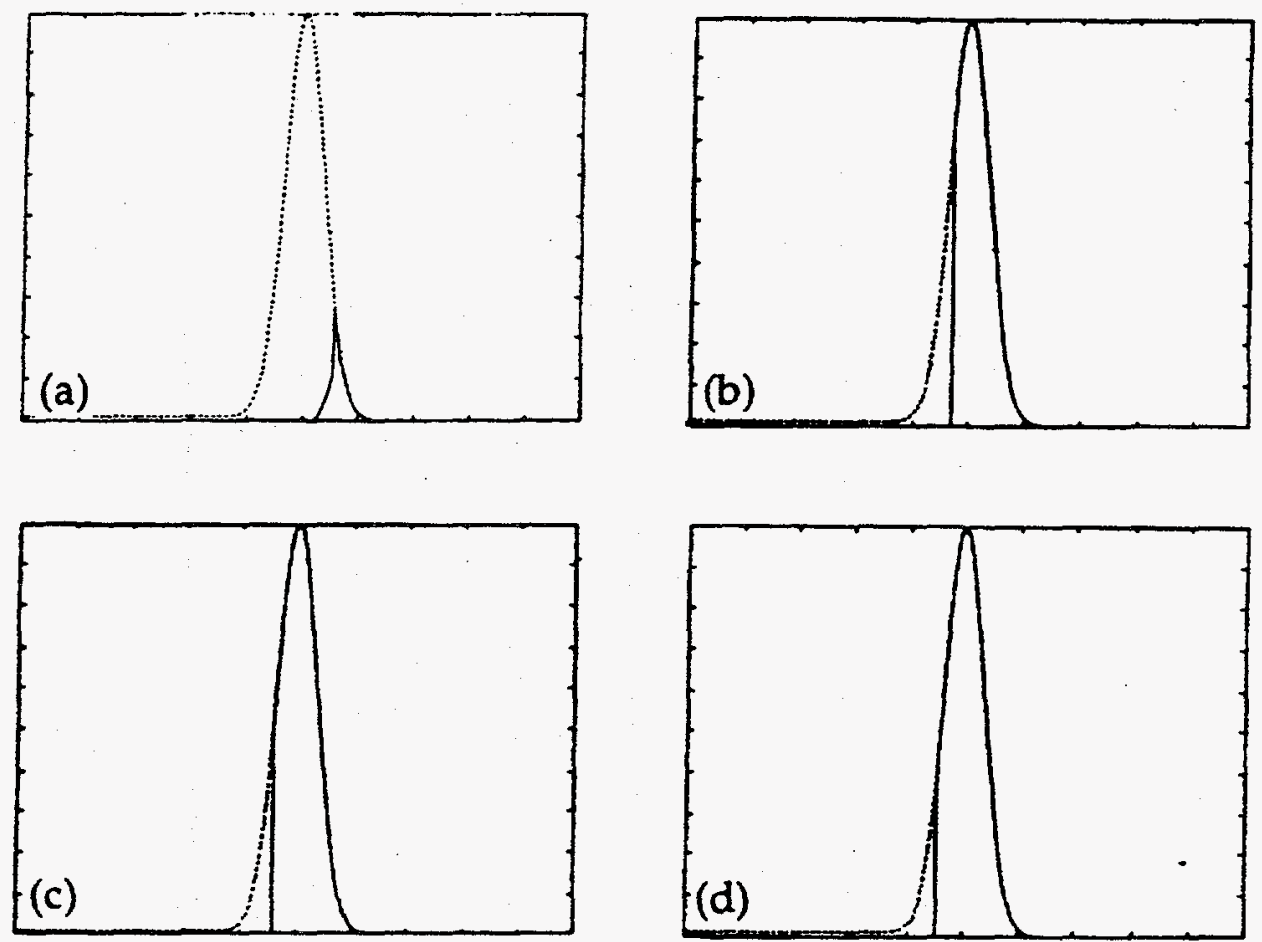

Figure 6.12: Plasma mirror model results for the intensities (a) $1.0 \times 10^{14} \mathrm{~W} / \mathrm{cm}^{2}(b)$ $5.0 \times 10^{15} \mathrm{~W} / \mathrm{cm}^{2}$ (c) $7.5 \times 10^{15} \mathrm{~W} / \mathrm{cm}^{2}$ (d) $1 \times 10^{16} \mathrm{~W} / \mathrm{cm}^{2}$.

In each plot, the incident pulse is overlaid as a dashed curve for comparison and the pulses are normalized to the peak intensity. The reflected pulse risetime is shorter than the incident pulse risetime in all cases except at the lowest intensities where the plasma barely reaches a critical density. The reflected pulse rising edge occurs earlier in time in a nonlinear fashion as the intensity is increased. The absolute time that the reflectivity rise appears relative to the incident pulse center is plotted in Figure 6.13. Only the pulse energy is increased. The pulsewidth and the area are kept constant. The pulse therefore gets sharper and its risetime gets shorter as the intensity is increased. 


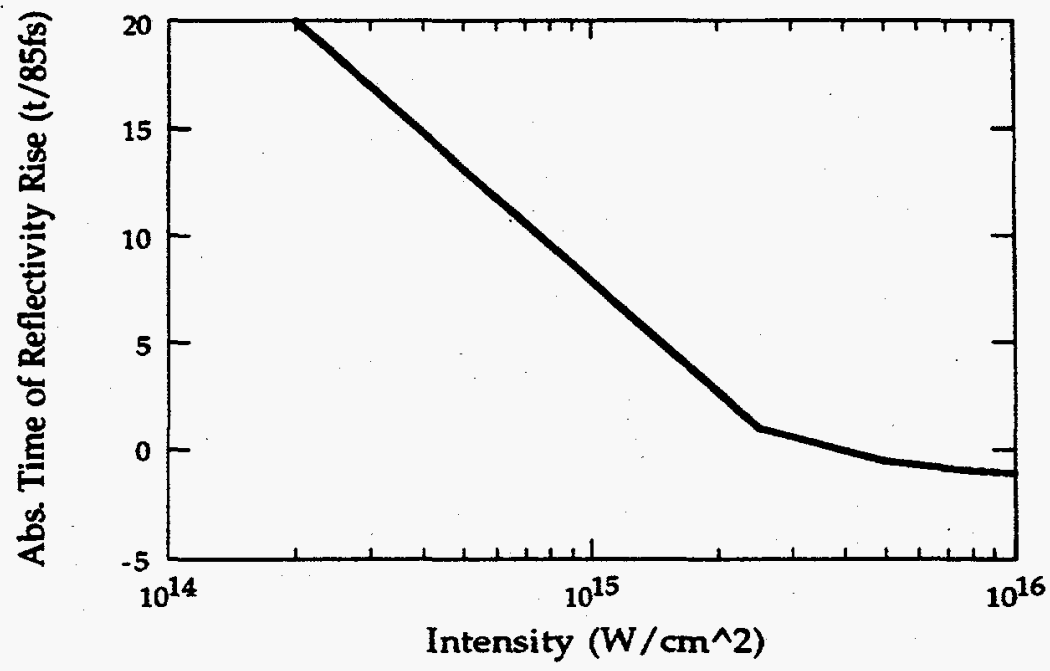

Figure 6.13: Absolute time of reflectivity rise versus intensity.

At high intensities, the time at which the mirror "switches on" does not shift much, because the pulse risetime does not change much as the intensity is increased. Thus, the integrated energy transmitted and used to create the plasma mirror does not change much at high intensities. This is the signature of an effect that depends on the peak intensity and not the total energy absorbed, i.e., it is a nonlinear, multiphoton effect. Looking at Figure 6.12 (a) again, however, we see that the pulse passed through the critical intensity (when the plasma mirror turns on) on the leading edge, but the reflective plasma did not form until the trailing edge. This means that the intensity is low enough that multiphoton processes are not the dominant ionization mechanism. The plasma forms instead by heating through inverse Bremsstrahlung - an integrated energy effect. Note that when the plasma forms in this more conventional, collisional or low-intensity, manner. the reflected pulse risetime is about the same as the incident pulse, not steeper as in the multiphoton case. 


\subsection{POSTPULSE SUPPRESSION}

In addition to the measured prepulse suppression above, ideas on postpulse suppression are proposed. First, a set of two plasma mirrors could be set up, one reflective and one transmissive (Figure 6.14). The prepulse would be eliminated in the standard reflective mirror arrangement discussed in this chapter. The postpulse would be removed by sending the unfocused beam through a second transmissive material and focusing the optically delayed pulse on the same spot to create a second plasma. The second plasma would be timed by an optical delay to form a critical surface just before the postpulse was transmitted from the pulse off the first mirror. The postpulse would be reflected or absorbed and only the clean femtosecond pulse would remain.

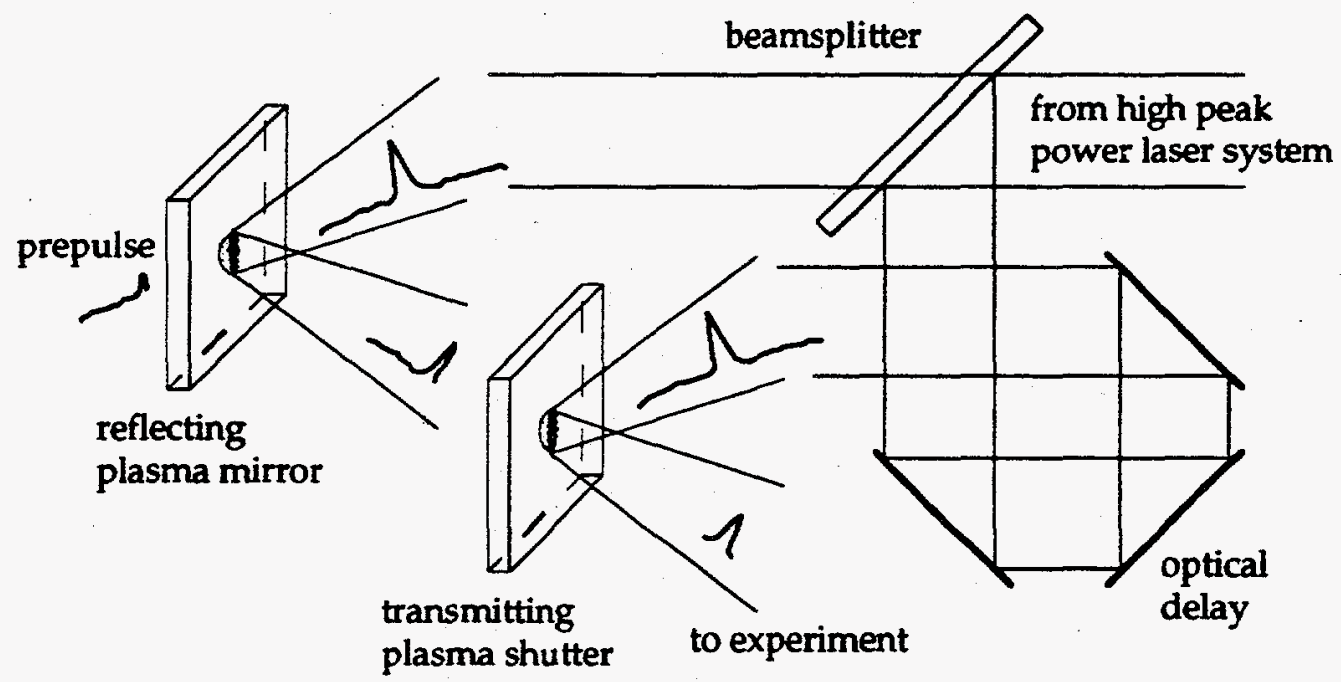

Figure 6.14: A proposed double plasma mirror set-up for eliminating prepulse and postpulse from an intense, ultra-short laser pulse.

A second postpulse suppression method, Figure 6.15, also follows the plasma mirror scheme, except an uncoated grating will be used as the target. The grating allows the prepulse to pass through as in the planar plasma mirror, but then forms a highly reflective, expanding plasma on the grating surface that coats the grating. The main pulse then reflects off the grating strongly in the zero and 
first orders as the plasma continues to expand. If the first order reflection is used, it is possible that the damage to the grating rulings due to the plasma expansion is large enough to cause a significant reduction in grating efficiency for first order reflection by the time the postpulse arrives at the reflective plasma surface. The postpulse is then reflected efficiently only in the zeroth order direction. The pulse reflected in the first order has significantly reduced prepulse and postpulse. To circumvent the use of expensive grating targets, gratings could be actively induced on flat targets by another laser $[200,201]$. This also offers the advantage of a capability for an adjustable grating spacing and depth.

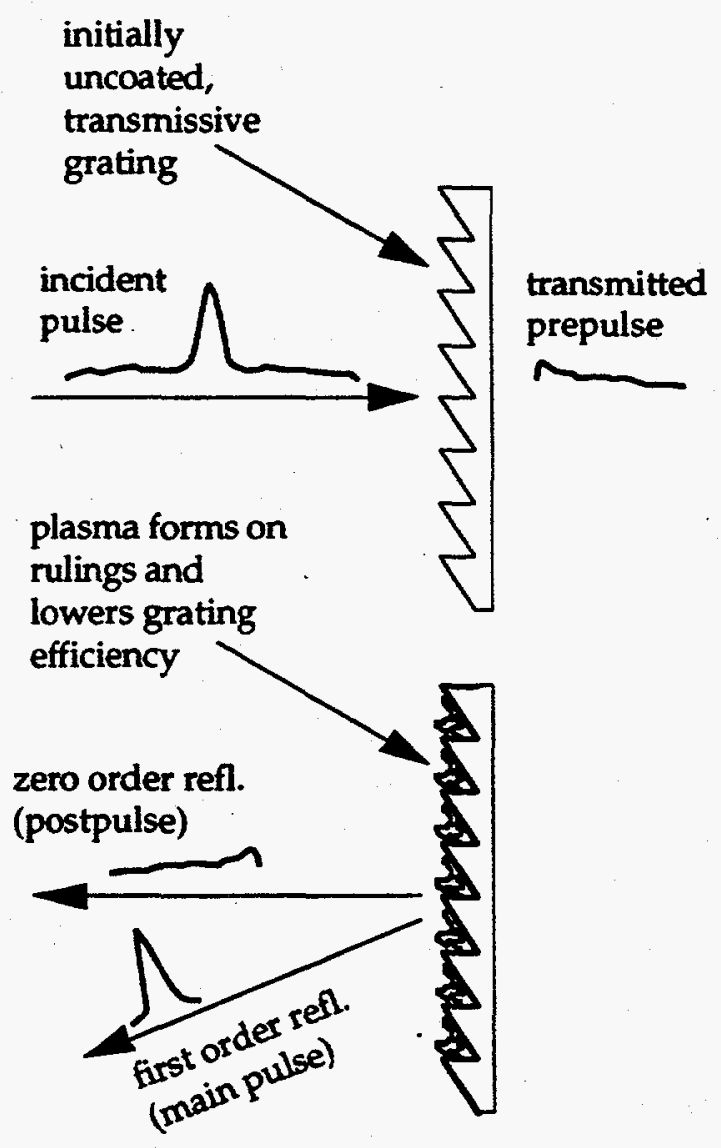

Figure 6.15: A proposed grating plasma mirror experiment for eliminating prepulse and postpulse from an intense, ultra-short laser pulse. 


\section{CHAPTER 7:}

\section{CONCLUSION}

This dissertation research characterized reflective properties of plasmas created by high-intensity, ultra-short laser pulses. Plasma formation and evolution features including ionization mechanisms, energy absorption processes and plasma dynamics were identified and quantified as inferred from timeintegrated or space- and time-resolved reflectivity measurements. A low-noise laser system was designed to generate high-intensity $\left(10^{16} \mathrm{~W} / \mathrm{cm}^{2}\right)$, ultra-short (130fs) optical pulses for unambiguous high-density plasma creation and probing.

Time-integrated data quantified the reflective response of several dissimilar materials subjected to a significant, nearly instantaneous increase in energy density. These experimental results represent the first data for reflectivity as a function of high intensities over such a broad range of materials which is critical to consistent, coherent analysis. Dramatic differences in intensitydependent self-reflectivity for metals $(\mathrm{Al}, \mathrm{Au})$, a semiconductor $(\mathrm{Si})$, and an insulator $\left(\mathrm{SiO}_{2}\right)$ improve the understanding of matter's reflective response under unique high-temperature, high-density conditions.

The response of metals ( $\mathrm{Al}, \mathrm{Au}$ ) emphasized absorption physics, because the entire pulse interacted with supercritical electron density. High-intensity $\left(\sim 10^{15}-10^{16} \mathrm{~W} / \mathrm{cm}^{2}\right)$ results were accurately explained by inverse Bremsstrahlung absorption with intensity-dependent modifications. Resonance absorption and vacuum heating, a new high-intensity effect, also contributed when $P$ polarization was used. Moreover, absorption and spectral shift measurements were used to infer plasma density, collisionality, temperature, average ionization 
state, expansion velocity, and scale length. Hydrodynamic plasma simulation results showed that at lower intensities, $-10^{12}-1015 \mathrm{~W} / \mathrm{cm}^{2}$, reflectivity predictions depend critically on an accurate transitional conductivity.

In contrast to the metals' reflectivity, the insulator $\left(\mathrm{SiO}_{2}\right)$ response emphasized ionization physics. Rapid, enormous changes in electron density governed reflectivity, because the reflectivity change from a cold, insulating solid to a supercritical plasma was more important than the absorption fraction. Rapid ionization in $\mathrm{SiO}_{2}$ is facilitated by a relatively new mechanism for plasma breakdown, tunneling ionization. Reflectivity data shows significant plasma formation at intensities where the predicted tunnel ionization rate exceeds the avalanche ionization rate. New evidence for collisional ionization suppression by strong free electron scattering from non-polar acoustic phonons through the acoustic deformation potential interaction also supports the use of tunnel ionization. This important departure from standard avalanche theory is used here, for the first time, to predict plasma formation characteristics in the ultrashort pulse regime. Results from a tunnel ionization model agreed well with reflectivity data from an $\mathrm{SiO}_{2}$ target.

A simple physical model demonstrated that a high-intensity, ultra-short laser pulses can produce a non-LTE plasma on its rising edge. The fundamental mechanism behind this nonequilibrium growth is the domination of photoionization over collisional or temperature-driven ionization. A temperaturedriven plasma maintains the relationship $\tau_{e q}<\tau_{i r o b r e m}=\tau_{\text {heating, }}$, so its ion states can equilibrate as the plasma is heated. A high-intensity field, however, produces a tunnel-ionized plasma with the relationship $\tau_{\text {tumnel }}<\tau_{e q}<\tau_{\text {ino brem. }}$. This difference means ionization occurs much faster than the ion states can relax to a Saha equilibrium state through collisional processes. The important timescales to compare are the photo-ionization time and the ionization state equilibration time, 
not the ion equilibration time and the time for electron heating by inverse Bremsstrahlung. At approximately solid densities, the electron-ion collision time is small enough to allow the plasma to form in LTE when field-induced processes are not considered. However, when the very rapid field ionization is considered, the initial field-driven stage cannot form in LTE. This is unique to the new regime of high-intensity, ultra-short pulse interactions.

Without detailed analysis, semiconductor, $\mathrm{Si}$, reflectivity data was shown to be the intermediate case for reflective response. It showed features of ionization-dependent, insulator-like, behavior at low intensity and nonlinear absorption, metal-like, behavior at the highest intensities.

The response of all materials tested converged to a common plasma state with a reflectivity $\sim 70-80 \%$ (S polarization) and $~ 55-60 \%$ (P polarization, $15^{\circ}$ ) at the highest intensities, $\sim 10^{16} \mathrm{~W} / \mathrm{cm}^{2}$. This was the first evidence of a materialindependent plasma reflectivity law which may be useful for predicting a nearly unexpanded materials' conductivity at high energy densities. It is confirmed by independent results at intensities up to $10^{18 \mathrm{~W}} / \mathrm{cm}^{2}$.

Spatially- and temporally-resolved reflectivity provided a direct measurement of the convolution of reflective plasma states distributed over the focal region of a laser-plasma interaction. Furthermore, reflectivity in the central plasma region gave the first direct indication of the transition between rapid plasma cooling from electron conduction and the appearance of significant hydrodynamic expansion. Reducing the temperature gradient, by lowering the pump intensity or introducing impurities, confirmed predictions that the plasma cooling rate from electron conduction is reduced below frontside probe observational levels.

The first direct evidence of prepulse suppression using an optical wavelength, ultra-fast plasma switch was presented. Using an $\mathrm{SiO}_{2}$ target at 
Brewster's angle, the contrast ratio was improved by a, detector limited, factor $>625$. Feasibility of prepulse suppression at pulse repetition rates up to $\sim 2 \mathrm{kHz}$ was demonstrated with a regenerating, liquid (ethylene glycol) target. In both the liquid and solid target experiments, focusability of the reflected beam was not sacrificed and some spatial filtering and beam smoothing due to a reflective profile in the plasma was seen. A pulsewidth decrease of $\sim 7-10 \%$ was seen in the reflected beam, however, no data on a steepened risetime was available due to the second-order nature of the measurement. A simple model was developed to study the effect of an ultra-fast plasma switch on a incident pulse. Results show a steepened risetime in the reflected pulse when peak intensity is high enough $\left(>10^{14} \mathrm{~W} / \mathrm{cm}^{2}\right)$ for MPI or tunneling ionization to create a critical density. Below this intensity, the model predicts that avalanche ionization produces a reflected pulse with a risetime on the order of the incident pulse. Finally, two schemes for reducing both prepulse and postpulse are proposed as future work in this area. 


\section{APPENDIXA:}

\section{RATE EOUATIONS FOR ION PRODUCTION}

The charge states of an atom are assumed to be produced sequentially by field ionization or by electron collisions. It is also assumed that the charge states recombine by three-body collisions in a sequential manner. This model ignores processes in which two or more electrons are simultaneously lost or acquired by an ion.

Let us denote the densities of the charge states $0,1,2, \ldots, Z$ for an atom of atomic number $Z$, as $n_{0}, n_{1}, n_{2}, \ldots, n_{Z}$ respectively. Furthermore let $\alpha_{0}, \alpha_{1}, \alpha_{2}, \ldots, \alpha_{z-1}$ and $\omega_{0}, \omega_{1}, \omega_{2}, \ldots, \omega_{z-1}$, represent, respectively, the avalanche ionization coefficients and the electron tunneling rates of charge states $0,1,2, \ldots, Z-1$. On the other hand, let the recombination coefficients $\rho_{0}, \rho_{1}, \rho_{2}, \ldots, \rho_{z-1}$ be labeled by the final charge states of the reverse reactions $n_{i+1}+2 e \Rightarrow n_{i}+e$. The rate equations for sequential ionization and recombination are

$$
\begin{aligned}
& \frac{d n_{0}}{d t}=-\left(\alpha_{0} n_{0} n_{e}-\rho_{0} n_{1} n_{e}^{2}+\omega_{0} n_{0}\right) \\
& \frac{d n_{1}}{d t}=-\left(\alpha_{1} n_{1} n_{e}-\rho_{1} n_{2} n_{e}^{2}+\omega_{1} n_{1}\right)+\left(\alpha_{0} n_{0} n_{e}-\rho_{0} n_{1} n_{e}^{2}+\omega_{0} n_{0}\right) \\
& \frac{d n_{2}}{d t}=-\left(\alpha_{2} n_{2} n_{e}-\rho_{2} n_{3} n_{e}^{2}+\omega_{2} n_{2}\right)+\left(\alpha_{1} n_{1} n_{e}-\rho_{1} n_{2} n_{e}{ }^{2}+\omega_{1} n_{1}\right)
\end{aligned}
$$

$$
\begin{aligned}
& \frac{d n_{z-1}}{d t}=-\left(\alpha_{z-1} n_{z-1} n_{e}-\rho_{z-1} n_{z} n_{e}^{2}+\omega_{z-1} n_{z-1}\right)+\left(\alpha_{z-2} n_{z-2} n_{e}-\rho_{z-2} n_{z-1} n_{e}^{2}+\omega_{z-2} n_{z-2}\right) \\
& \frac{d n_{\mathrm{Z}}}{d t}=\quad+\left(\alpha_{\mathrm{Z}-1} n_{\mathrm{Z}-1} n_{e}-\rho_{\mathrm{Z}-1} n_{\mathrm{Z}} n_{e}{ }^{2}+\omega_{\mathrm{Z}-1} n_{\mathrm{Z}-1}\right)
\end{aligned}
$$


The conservation of charge closes Eqs. (A1) by relating the electron density to the charge state densities

$$
n_{e}=n_{1}+2 n_{2}+3 n_{3}+\ldots+z n_{z}
$$

Equation (A1) also conserves particles,

$$
n_{0}+n_{1}+n_{2}+\ldots+n_{z}=N_{0}
$$

since the right hand side sums to zero. $N_{0}$ in Eq. (A3) represents the initial density of atoms.

For the case of $Z=1$, these equations simplify. All coefficients in Eq. (A1) are zero except $\alpha_{0}, \rho_{0}$, and $\omega_{0}$. The electron density $n_{e}$ is equal to $n_{1}$ because of Eq. (A2). Then by Eq. (A3), $n_{0}$ is equal to $N_{0}-n_{e}$. This last result can be substituted into the first equation of Eq. (A1) to give Eq. (4.16). 


\section{APPENDIX B:}

\section{LASER-ASSISTED AVALANCHE COEFFICIENTS}

The avalanche coefficient for impact ionization can depend on laser intensity through the dependence of either the electron distribution function or the cross section, because it is the integral of the product of both. In this appendix, we derive the intensity dependence of the avalanche coefficient which stems from the intensity-dependent distribution function. The intensity dependence due to the collision cross section is not examined, because a closed form expression has not been derived for it.

The distribution function is assumed to be governed by Krook's approximation [202] of the Boltzmann equation

$$
\frac{\partial f}{\partial t}+v \frac{\partial f}{\partial x}+a \frac{\partial f}{\partial v}=\frac{1}{\tau}\left(f-f_{t h}\right)
$$

where $\mathbf{a}$ is the acceleration $e E / m, \tau$ is the relaxation time related to $\lambda_{m p p}$ the mean free path as $\left(2 m \lambda_{m f p} /|e E|\right)^{1 / 2}$, and $f_{t h}=\pi^{-3 / 2} v_{t h}{ }^{-3} \exp \left(-v^{2} / v_{t h}{ }^{2}\right)$ is the Boltzmann distribution function. We assume $E$ to be a static field in the $z$ direction.

Equation (B1) can be integrated analytically. At infinite times, the general solution relaxes to the spatially homogeneous and time-independent distribution

$$
\begin{aligned}
f & =\left(\sqrt{\pi} v_{t h}\right)^{-3} \sqrt{\pi / 4}\left(v_{t h} / a \tau\right) \\
& \times \exp \left(-v_{p e r p}^{2} / v_{t h}^{2}\right) \exp \left(-v_{z} / a \tau+v_{t h}^{2} / 4 a^{2} \tau^{2}\right)\left\{\operatorname{erf}\left(v_{z} / v_{t h}-v_{t h} / 2 a \tau\right)-1\right\}
\end{aligned}
$$

where $v_{z}$ and $v_{\text {perp }}$ are respectively the velocity components parallel and perpendicular to $E$. According to Eq. (B2), the field shifts the center of the distribution to $v_{z} \sim a \tau$ approximately and skews the distribution upfield.

In order to integrate the defining expression $\int \sigma o f d^{3} v$ for the avalanche coefficient analytically, the impact ionization cross section is assumed to be 
spherically symmetric and to be $\sigma=\sigma^{\prime}\left(E-I_{p}\right) \exp \left(-\gamma\left(E^{1 / 2}-I_{p}^{1 / 2}\right)\right)$. This form for the cross section has the same salient features as Zel'dovich's and Raizer's parametrization [151] used to derived Eq. (4.19).

When the field shifts the distribution function across the cross section, it enhances the avalanche coefficient by increasing the overlap between the distribution function and the cross section. The field shift in Eq. (B2) can be made more self-evident by approximating the $v_{z}$ portion of the distribution function in terms of its first three cumulates [203]. The result is

$$
f=\left(\sqrt{\pi} v_{t h}\right)^{-3} \exp \left(-v_{\text {perp }}^{2} / v_{t h}^{2}\right) \exp \left(-\left(v_{z}-v_{0}\right)^{2} / v_{t h}^{2}\right)
$$

where the field shifted center $v_{0}$ of the distribution is equal to $a \tau$. The essence of Eq. (B3) is that the field moves the center of the distribution function upfield by the amount $a \tau$.

Since the cross section is spherically symmetric, the angular integration of Eq. (B3) gives

$$
f=\left(\sqrt{\pi} v_{t h}\right)^{-1}\left(2 / v_{0} v\right) \exp \left(-\left(v^{2}+v_{0}^{2}\right) / v_{t h}^{2}\right) \sinh \left(2 v_{0} v / v_{t h}^{2}\right)
$$

Therefore the field dependence in laser-assisted avalanche coefficient can be expressed as

$$
\alpha=\frac{2 \sigma^{\prime} k T_{e} v_{t h}}{\sqrt{\pi}} \exp \left(2 \gamma_{t} v_{l}+\gamma_{t}^{2}\right)\left[\psi_{1} \exp \left(-v_{\beta}^{2}\right)-\psi_{2} \operatorname{erfc}\left(\mathrm{v}_{\beta}\right)\right]
$$

where

$$
\begin{aligned}
\psi_{1}= & \left(2+2 v_{F}^{2}+v_{F}^{4}\right)+\left(10 \gamma_{t}^{2}-v_{I}^{2}\right)\left(1+v_{F}^{2}\right)+\left(5 \gamma_{t}^{4}-3 \gamma_{t}^{2} v_{I}^{2}\right) \\
& -5 \gamma_{t} v_{F}\left(3 / 2+v_{F}^{2}\right)-v_{F}\left(10 \gamma_{t}^{3}-3 \gamma_{t}^{2} v_{I}^{2}\right),
\end{aligned}
$$

and

$$
\psi_{2}=\sqrt{\pi}\left(15 \gamma_{t} / 4+5 \gamma_{t}^{3}-3 \gamma_{t}^{2} v_{I}^{2} / 2+\gamma_{t}^{5}-\gamma_{t}^{3} v_{I}^{2}\right)
$$

The dimensionless variables in Eqs. (B5), (B6) and B(7) are: $\gamma_{t}=\gamma\left(k T_{e} / 4\right)^{1 / 2}$, $v_{I}=\left(I_{p} / k T_{e}\right)^{1 / 2}$ and $v_{F}=v_{I}+\gamma_{t}+|e E \tau| / m v_{t h}$. The pre-exponential factor in Eq. 
(B5), $\sigma^{\prime} k T_{e} \nabla_{t h}$ is of order $\omega_{a u} / n_{a u}$. Therefore Eq. (B5) and Eq. (4.19), Zel'dovich's and Raizer's avalanche coefficient, are similar in magnitude.

Equation (B5) is plotted against the laser intensity for several temperatures in Figure B1. At room temperature (not shown in figures), the laser enhances the avalanche coefficient by more than 230 orders of magnitude when the intensity is

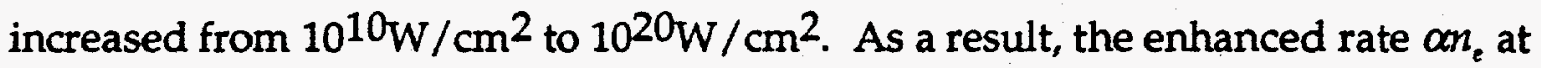
$0.025 \mathrm{eV}$ can be larger than the tunneling rate at $10^{20} \mathrm{~W} / \mathrm{cm}^{2}$ if the electron density is comparable to $n_{a u}$. On the other hand, the avalanche coefficient is insensitive to the laser when the temperature is approximately $10 I_{p^{*}}$ This insensitivity is illustrated in Figure $B 1(c)$. Beyond this temperature of $10 I_{p}$, the field causes $\alpha$ to decrease by reducing the overlap of $f$ and $\sigma$. This decrease is illustrated in Figure B1(d).

In summary, the laser affects the avalanche coefficient by changing the overlap between the electron distribution function and the impact ionization cross section. In order for the laser enhancement to be appreciable, the laser must shift the distribution into the significant part of the cross section. The conditions under which this enhancement occurs are: (1) the temperature must be lower than $I_{p} / k$ so that thermal distribution's penetration of the cross section is negligible, and (2) the laser field must be larger than $I_{p} / \lambda_{m g p}$ so that field shift of the distribution's center is beyond the threshold of the cross section. However at very high fields or very high temperatures, the avalanche coefficient decreases with increasing intensity or increasing temperature because the overlap of $f$ and $\sigma$ decreases in those regimes. 

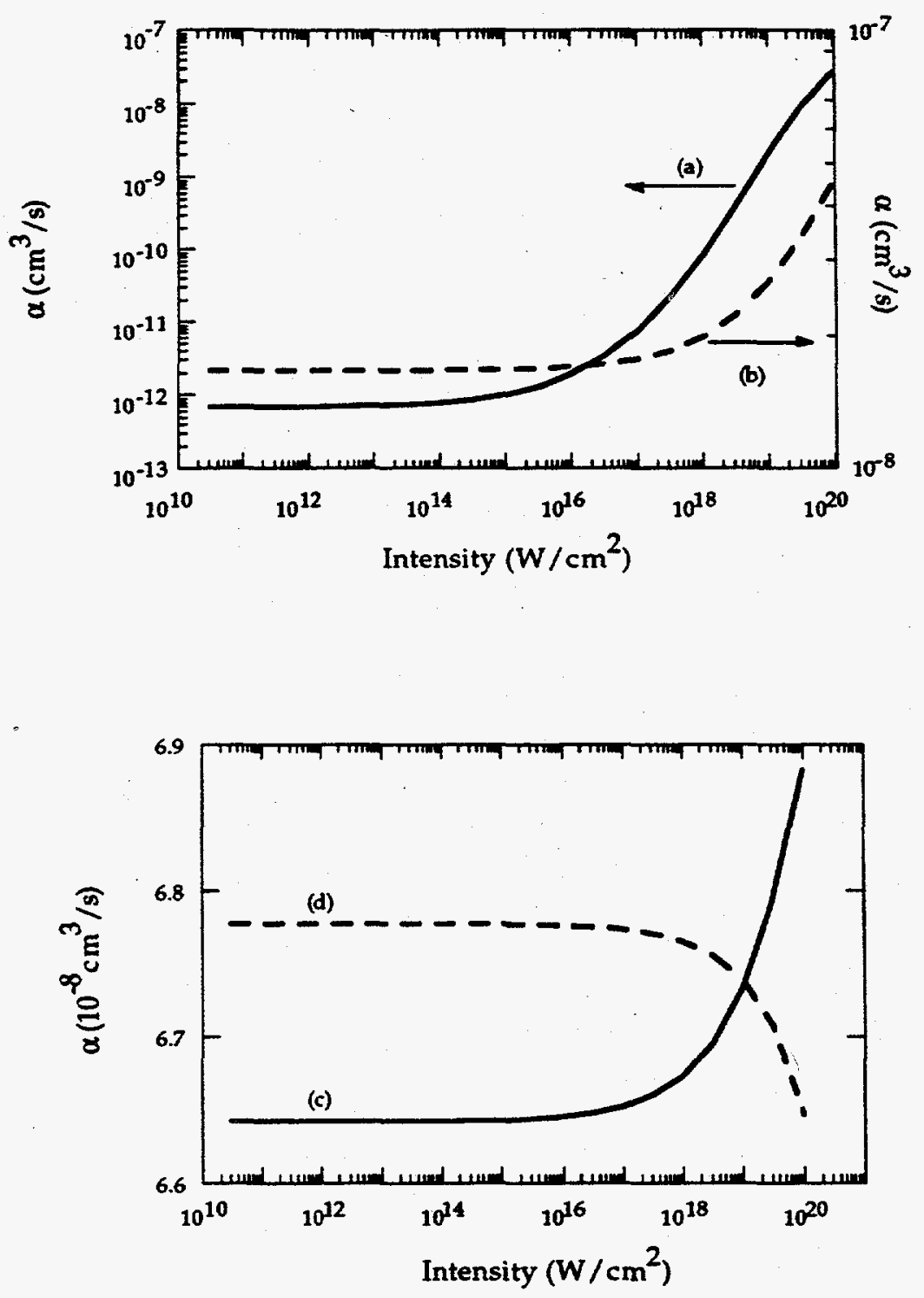

Figure B1: (a) $\alpha$ as a function of intensity for $T_{c}=1.4 \mathrm{eV}$ (solid). The field increases $\alpha$ by 4 orders of magnitude for an intensity increase of 10 orders of magnitude. (b) The same as Figure B1(a) except $T_{e}$ is increased to $14 \mathrm{eV}$ (dash). Relative increase is only a factor of 3 ooer ten orders of intensity. (c) The same as Figure B1(a) except $T_{\varepsilon}$ is increased to $79 \mathrm{eV}$ (solid). Notice $\alpha$ is insensitive to the field increase. Beyond this temperature $\sim 10 I_{p}$, the field causes $\alpha$ to decrease by reducing the overlap of the electron distribution function, $f$, and the cross section $\sigma$. (d) The same as Figure B1(a) except $T_{e}$ is increased to $140 \mathrm{eV}$ (dash). The field causes $\alpha$ to decrease near $T_{c}(140 \mathrm{eV})>10 I_{p}$. 


\section{REFERENCES}

[1] S.A. Akhmanov, V.A. Vyslouch, and A.S. Chirkin, Optics of Femtosecond Laser Pulses. (American Institute of Physics, New York, 1992).

[2] J.D. Jackson, Classical Electrodynamics. 2nd. Ed. John Wiley and Sons, New York, 1975).

[3] H.M. Milchberg, R.R. Freeman, and S.C. Davey, Phys. Rev. Lett. 61, 2364 (1988).

[4] D.H. Reitze, H. Ahn, and M.C. Downer, Phys. Rev. B 45, 2677 (1992).

[5] J. Sheffield, Plasma Scattering of Electromagnetic Radiation. (Academic Press, New York, 1975).

[6] M.M. Murnane, Sub-Picosecond Laser Produced Plasmas. Ph.D. Dissertation (University of California, Berkeley, 1989).

[7] R.C. Elton, X-Ray Lasers. (Academic Press, Inc., San Diego, 1990).

[8] S.L. Chin and P. Lambropoulos, ed. Multiphoton Ionization of Atoms. (Academic Press, Inc., New York, 1984). 
[9] M. Gavrila, ed. Atoms in Intense Laser Fields. Advances in Atomic, Molecular, and Optical Physics, ed. B. Bederson and D. Bates. Vol. 1, (Academic Press, Inc., New York, 1992).

[10] Keldysh, Sov. Phys. JETP 20, 1307 (1965).

[11] N.H. Burnett and P.B. Corkum, J. Opt. Soc. Am. B 6, 1195 (1989).

[12] P. Amendt, D.C. Eder, and S.C. Wilks, Optically-ionized plasma recombination $x$-ray lasers. (Lawrence Livermore National Laboratory, 1991).

[13] M.M. Murnane, H.C. Kapteyn, and R.W. Falcone, Phys. Rev. Lett. 62 , 155 (1989).

[14] N. Bloembergen, IEEE J. Quantum Electron. QE-10, 375 (1974).

[15] A.J. Glass and A.H. Guenther, Appl. Opt. 12, 637 (1973).

[16] P.W. Milonni and J.H. Eberly, Lasers. John Wiley \& Sons, New York, 1988).

[17] W.E. White, H. Nathel, W. Tulloch, and D.M. Gold, A Compact Laser System for Producing Sub-100fs Laser Pulses with Millijoule Energies, in American Physical Society: Division of Plasma Physics Annual Meeting, edited by American Physical Society (Cincinnati, Ohio, 1990).

[18] P. Maine, et al., IEEE J. Quantum Electron. QE-24, 398 (1988). 
[19] P.B. Corkum and R.S. Taylor, IEEE J. Quantum Elecron. QE-18, 1962 (1962).

[20] G. Mourou and D. Umstadter, Phys. Fluids B 4, 2315 (1992).

[21] A.E. Siegman, Lasers. (University Science Books, Mill Valley, CA, 1986).

[22] H.W. Mocker and R.J. Collins, Appl. Phys. Lett. 7, 270 (1965).

[23] E.G. Arthurs, D.J. Bradley, and A.G. Roddie, Appl. Phys. Lett. 20, 125 (1972).

[24] E.P. Ippen, C.V. Shank, and A. Dienes, Appl. Phys. Lett. 21, 348 (1972).

[25] R.L. Fork, B.I. Greene, and C.V. Shank, Appl. Phys. Lett. 38, 671 (1981).

[26] F. Shimizu, Phys. Rev. Lett. 19, 1097 (1967).

[27] A.C. Cheung, D.M. Rank, R.Y. Chiao, and C.H. Townes, Phys. Rev. Lett. 20, 786 (1968).

[28] R.W. Boyd, Nonlinear Optics. (Academic Press, Inc., San Diego, 1992).

[29] R.G. Brewer, Phys. Rev. Lett. 19(1967). 
[30] Y.R. Shen, The Principles of Nonlinear Optics. Uohn Wiley \& Sons, Inc., New York, 1984).

[31] J.A. Valdmanis, R.L. Fork, and J.P. Gordon, Opt. Lett. 10, 131 (1985).

[32] M.M. Murnane, et al.in Tech. Dig. Opt. Soc. Amer. Ann. Meet., edited by OSA (Toronto, Canada, 1993).

[33] C.V. Shank, et al., Appl. Phys. Lett. 40, 761 (1982).

[34] R.L. Fork, C.V. Shank, C. Hirlimann, and R. Yen, Opt. Lett. 8, 1 (1983).

[35] E.P. Treacy, IEEE J. Quantum Electron. QE-5, 454 (1969).

[36] R.L. Fork, Opt. Lett. 11, 629 (1986).

[37] O.E. Martinez, J. Opt. Soc. Am. B. 3, 929 (1986).

[38] R.L. Fork, C.H. Brito Cruz, P.C. Becker, and C.V. Shank, Opt. Lett. 12, 483 (1987).

[39] W.H. Knox, et al., Appl. Phys. Lett. 46, 1120 (1985).

[40] P. Beaud, W. Hodel, B. Zysset, and H.P. Weber, IEEE J. Quantum Electron. QE-23, 1938 (1987). 
[41] A.S. Gouveia-Neto, A.S.L. Gomes, and J.R. Taylor, Opt. Commun. 64, 383 (1987).

[42] P. Sprangle and E. Esarey, Phys. Fluids B 4, 2241 (1992).

[43] W. Koerchner, Solid-State Laser Engineering. (Springer-Verlag, Berlin, 1988).

[44] A. Sullivan, et al., Opt. Lett. 16, 1406 (1991).

[45] W.E. White, et al., Opt. Lett. 17, 1 (1992).

[46] F.G. Patterson, R. Gonzales, and M.D. Perry, Opt. Lett. 16, 1107 (1991).

[47] K. Yamakawa, H. Shiraga, Y. Kato, and C.P.J. Barty, Opt. Lett. 16, 1593 (1991).

[48] D.J. Erskine, A.J. Taylor, and C.L. Tang, Appl. Phys. Lett. 45, 54 (1984).

[49] J.A. Kash, J.C. Tsang, and J.M. Hwam, Phys. Rev. Lett. 54, 2151 (1985).

[50] W.H. Knox, et al., Phys. Rev. Lett. 54, 1306 (1985).

[51] D. Krokel, D. Grischkowsky, and M.B. Ketchen, Appl. Phys. Lett. 54, 1049 (1989).

[52] B.I. Green and R.C. Farrow, J. Chem. Phys. 78, 3336 (1983). 
[53] J.L. Martin, et al., Proc. Natl. Acad. Sci. 80, 173 (1983).

[54] O.E. Martinez, R.L. Fork, and J.P. Gordon, Opt. Lett. 9, 156 (1984).

[55] H.A. Haus, J. Appl. Phys. 46, 3049 (1975).

[56] H.A. Haus, J. Quantum Electron. QE-11, 736 (1975).

[57] H.A. Haus, C.V. Shank, and E.P. Ippen, Opt. Commun. 15, 29 (1975).

[58] H.A. Haus, J. Quantum Electron. QE-12, 169 (1976).

[59] H.A. Haus, H. Statz, and I.W. Smith, IEEE J. Quantum Electron. QE21, 78 (1985).

[60] A.M. Johnson and W.M. Simpson, Opt. Lett. 8, 554 (1983).

[61] L.M. Davis, J.D. Harvey, and J.M. Pearl, Opt. Commun. 50, 49 (1984).

[62] M.M. Murnane and R.W. Falcone, J. Opt. Soc. Am. B 5, 1573 (1988).

[63] J.A. Valdmanis and R.L. Fork, IEEE J. Quantum Electron. QE-22, 112 (1986).

[64] W. Demtroeder, Laser Spectroscopy Basic Concepts and Instrumentation. Third Ed. (Springer-Verlag, Berlin, 1988). 
[65] J.J. Fontaine, W. Dietel, and J.C. Diels, IEEE J. Quantum Electron. QE19,1467 (1983).

[66] R.S. Miranda, G.R. Jacobovitz, C.H.B. Cruz, and M.A.F. Scarparo, Opt. Lett. 11, 224 (1986).

[67] W.B. Jiang, D.C. Sun, and F.M. Li, Opt. Commun. 64, 449 (1987).

[68] W. Rudolph, Opt. Quantum Electron. 16, 541 (1984).

[69] W. Rudolph and B. Wilhelmi, Appl. Phys. B. 35, 37 (1984).

[70] W. Rudolph and B. Wilhelmi, Opt. Commun. 49, 371 (1984).

[71] D. Khulke, W. Rudolph, and B. Wilhelmi, IEEE J. Quantum Electron. QE-19, 526 (1983).

[72] D. Khulke and W. Rudolph, Opt. and Quantum Electron. 16, 57 (1984).

[73] O.E. Martinez, R.L. Fork, and J.P. Gordon, J. Opt. Soc. Am. B 2, 753 (1985).

[74] R.L. Fork, O.E. Martinez, and J.P. Gordon, Opt. Lett. 9, 150 (1984). 
[75] CPM-1 Colliding Pulse Mode-Locked Dye Laser Kit Preliminary Manual. (Clark Instrumentation Inc., New York, 1987).

[76] D. Nickel, D. Kuhlke, and D. von der Linde, Opt. Lett. 14, 36 (1989).

[77] W.H. Knox, M.C. Downer, R.L. Fork, and C.V. Shank, Opt. Lett. 9, 552 (1984).

[78] Bowtie Amplifier and Continuum Generator. (Clark Instrumentation Inc., New York, 1987).

[79] D.S. Bethune, Appl. Opt. 20, 1897 (1981).

[80] E.L. Steele, J. Appl. Phys. 36, 348 (1965).

[81] L.M. Frantz and J.S. Nodvik, J. Appl. Phys. 34, 2346 (1963).

[82] R. Bellmann, G. Birnbaum, and W.G. Wagner, J. Appl. Phys. 34, 780 (1963).

[83] F. Hopf, High Energy Lasers and Their Applications. (Addison-Wesley, Reading, MA, 1974).

[84] P.G. Kriukov and V.S. Letokhov, Laser Handbook 1. (North-Holland, Amsterdam, 1972). 
[85] E.L. Steele, Optical Lasers in Electronics. (John Wiley \& Sons, New York, 1968).

[86] E.P. Ippen and C.V. Shank, Techniques for Measurement, in Ultrashort Light Pulses Picosecond Techniques and Applications, S.L. Shapiro, Editor. (SpringerVerlag, Berlin, 1977) p. 83.

[87] D.H. Auston, IEEE J. Quantum Electron. QE-7, 465 (1971).

[88] R.J. Harrach, Appl. Phys. Lett. 14, 148 (1969).

[89] R. Wyatt and E.E. Marinero, Appl. Phys. 25, 297 (1981).

[90] G. Szabo, Z. Bor, and A. Muller, Opt. Lett. 13, 746 (1988).

[91] S.M. Saltiel, K.A. Stankov, and P.D. Yankov L. I., Appl. Phys. B 40, 25 (1986).

[92] O.L. Bourne and A.J. Alcock, Rev. Sci. Instrum. 57, 2979 (1986).

[93] F. Salin, P. Georges, G. Roger, and A. Brun, Appl. Opt. 26, 4528 (1987).

[94] C.E. Max, Theory of the Coronal Plasma in Laser Fusion Targets. (Lawrence Livermore National Laboratory, 1982).

[95] V.L. Ginzburg, The Propagation of Electromagnetic Waves in Plasmas. 2nd. Ed. (Pergamon Press, New York, 1970). 
[96] W.L. Kruer, The Physics of Laser Plasma Interactions. (Addison-Wesley Publishing Co., New York, 1988).

[97] J. Cooper, Plasma Spectroscopy. (Imperial College London, London, 1966).

[98] S.C. Rae and K. Burnett, Phys. Rev. A 44, 3835 (1991).

[99] R.D. Jones and K. Lee, Phys. Fluids 25, 2307 (1980).

[100] A.B. Langdon, Phys. Rev. Lett. 44, 575 (1980).

[101] L. Schlessinger and J. Wright, Phys. Rev. A 20, 1934 (1979).

[102] G.J. Pert, J. Phys. A 5, 506 (1972).

[103] F.V. Bunkin and M.V. Fedorov, Sov. Phys. JETP 22, 844 (1966).

[104] V.P. Silin, Sov. Phys. JETP 20, 1510 (1965).

[105] S. Rand, Phys. Rev. B 136, 231 (1964).

[106] L.D. Landau and E.M. Lifshitz, Quantum Mechanics. 3rd. Ed. (Pergamon Press, Oxford, 1977). 
[107] A.M. Perelomov, V.S. Popov, and M.V. Terent'ev, Sov. Phys. JETP 23, 924 (1966).

[108] M.V. Ammosov, N.B. Delone, and V.P. Krainov, Sov. Phys. JETP 64, 1191 (1986).

[109] L.V. Keldysh, Sov. Phys. JETP 6(33), 763 (1958).

[110] B. Chang, P.R. Bolton, and D.N. Fittinghoff, Phys. Rev. A (to be published).

[111] D.N. Fittinghoff, et al., Ion yields from strong optical field ionization experiments using 100 femtosecond laser pulses, in Short-Pulse High-Intensity Lasers and Applications, edited by H.A. Baldis, Proc. SPIE 1413 (Los Angeles, California, 1991).

[112] V.L. Ginzburg, Izvestiya Akademii nauk SSSR, Seriya fizicheskaya 8, 76 (1944).

[113] C.G. Darwin, Proc. of the Royal Society 182, 152 (1943).

[114] R. Fedosejevs, et al., Appl. Phys. B 50, 79 (1990).

[115] L. Spitzer, Physics of Fully Ionized Gases. 2nd. Ed. (Interscience Publisers, New York, 1962). 
[116] O.L. Landen, B.T. Vu, D.G. Stearns, and W.E. Alley, Hydrodynamic eoolution of picosecond laser-plasmas, in SPIE Short-Pulse High-Intensity Lasers and Applications, edited by SPIE 1991).

[117] M. Chaker, et al., Phys. Fluids B 3, 167 (1990).

[118] D.H. Reitze, Femtosecond Dynamics of Phase Transitions in Carbon and Silicon. Ph.D. Dissertation (Univerisity of Texas at Austin, 1990).

[119] T.P. Hughes, Plasmas and Laser Light. John Wiley and Sons, Inc., New York, 1975).

[120] D. Price and R.M. More, personal communication.

[121] K.G. Estabrook and W.L. Kruer, (LLNL, 1986).

[122] K.G. Estabrook, E.J. Valeo, and W.L. Kruer, Phys. Fluids 18, 1151 (1975).

[123] D.W. Forslund, et al., Phys. Rev. A 11, 679 (1975).

[124] F. Brunel, Phys. Rev. Lett. 59, 52 (1987).

[125] Gibbon and Bell, Phys. Rev. Lett. 68, 1535 (1992).

[126] T. Holstein, Phys. Rev. 88, 1427 (1952). 
[127] P.J. Catto and M. More, Phys. Fluids 20, 704 (1977).

[128] P. Mulser, S. Pfalzner, and F. Cornolti, ed. Laser Interactions with Matter. ed. G. Velarde, E. Minguez, andJ.M. Perlado. (World Scientific, Singapore, 1989).

[129] E.G. Gamaliy and R. Dragila, Phys. Rev. A 42, 929 (1990).

[130] W. Rozmus and V.T. Tikhonchuk, Phys. Rev. A 42, 7401 (1990).

[131] W.L. Kruer and K. Estabrook, Phys. Fluids 28, 430 (1985).

[132] E.S. Weibel, Phys. Fluids 10, 741 (1967).

[133] R.M. More, Atomic Physics in Inertial Confinement Fusion. (Lawrence Livermore National Laboratory, 1981).

[134] O.L. Landen and W.E. Alley, Phys. Rev. A 46, 5089 (1992).

[135] H.M. Milchberg and R.R. Freeman, Phys. Rev. A 41, 2211 (1990).

[136] W.M. Wood, C.W. Siders, and M.C. Downer, Phys. Rev. Lett. 67, 3523 (1991).

[137] D.W. Forslund, J.M. Kindel, K. Lee, and E.L. Lindmann, Phys. Rev. Lett. 36, 35 (1976). 
[138] I. Clark, Bowtie Amplifier and Continuum Generator. (Clark Instrumentation Inc., New York,

[139] R. Cauble, F.J. Rogers, and W. Rozmus, Electrical Resistivity of Strongly Coupled Plasmas in Intense Fields, in Strongly Coupled Plasma Physics, S. Ichimaru, Editor. (North-Holland, Amsterdam, 1990)

[140] S. Ichimaru, Rev. Mod. Physics 54, 1017 (1982).

[141] F.J. Rogers, H.E. DeWitt, and D.B. Boercker, Phys. Lett. 82A, 331 (1981).

[142] M.W.C. Dharma-wardana and F. Perrot, Phys. Lett. A 163, 223 (1992).

[143] Y.T. Lee and R.M. More, Phys. Fluids 27, 1273 (1984).

[144] P. Celliers, Reflectivity of Intense Femtosecond Laser Pulses from a Simple Metal (Livermore, CA, 1993).

[145] M. Baus, J.P. Hansen, and L. Sjogren, Phys. Lett. 82A, 180 (1981).

[146] G.B. Zimmerman and W.L. Kruer, Comments Plasma Phys. Controlled Fusion 2, 51 (1975). 
[147] W.E. Alley, Modeling the absorption of intense, short laser pulses in steep density gradients, in Short-Pulse High-Intensity Lasers and Applications, edited by H.A. Baldis, SPIE (Los Angeles, CA, 1991).

[148] H.E. Elsayed-Ali, T.B. Norris, M.A. Pessot, and G.A. Mourou, Phys. Rev. Lett. 58, 1212 (1987).

[149] G.L. Eesley, Phys. Rev. B 33, 2144 (1985).

[150] J.G. Fujimoto, J.M. Liu, E.P. Ippen, and N. Bloembergen, Phys. Rev. Lett. 53, 1837 (1984).

[151] Y.A. Zel'dovich and Y.P. Raizer, Physics of Shock Waves and HighTemperature Hydordynamic Phenomena. 1 (Academic Press, New York, 1966).

[152] R.J. Harrach, Theory for Laser-Induced Breakdown Over a Vaporizing Target Surface. (Lawrence Livermore National Laboratory, 1977).

[153] M. Newstein and N. Solimene, IEEE J. Quant. Elec. QE-17, 2085 (1981).

[154] L.D. Landau and E.M. Lifshitz, A hydrogen atom in an electric field, in Quantum Mechanics (Non-Relativistic Theory). (Pergamon Press, Oxford, 1965) p. 673.

[155] G. Gibson, T.S. Luk, and C.K. Rhodes, Phys. Rev. A 41, 5049 (1990). 
[156] P. Braunlich, et al., Non-Avalanche Dielectric Breakdown in Wide-BandGap Insulators at DC and Optical Frequencies, in Laser Induced Damage in Optical Materials: Boulder Damage Symposium, edited by H.E. Bennet, et al., SPIE (Boulder, CO, 1989).

[157] S. Jones, et al., Opt. Eng. 28, 1039 (1989).

[158] D. Arnold and E. Cartier, Theory and modeling of Laser-induced freeelectron heating in wide-gap solids, in Laser Induced Damage in Optical Materials: Boulder Damage Symposium, edited by H.E. Bennet, et al., SPIE (Boulder, CO, 1992).

[159] M.V. Fischetti, et al., Phys. Rev. B 31, 8124 (1985).

[160] B.K. Ridley, Quantum Processes in Semiconductors. (Clarendon Press, Oxford, 1988).

[161] H. Froehlich, Proc. R. Soc. London Ser. A 188, 521 (1947).

[162] F. Seitz, Phys. Rev. 76, 1376 (1949).

[163] A. Caruso and R. Gratton, Interaction of Short Laser Pulses with Solid Materials. (Pergamon Press, New York, 1969).

[164] M. Abramowitz and I.A. Stegun, Section 9.6 Modified Bessel Functions I and $K$, in Handbook of Mathematical Functions, M. Abromowitz and I.A. Stegun, Editor. (Dover Publications, Inc., New York, 1964) p. 1046. 
[165] M. Born and E. Wolf, Priciples of Optics. 5th Ed. (Pergamon Press, New York, 1975).

[166] G.R. Fowels, Introduction to Modern Optics. (Holt, Rinehart and Winston Inc., New York, 1975).

[167] X.Y. Wang, Opt. Lett. 17, 1450 (1992).

[168] D. Price, personal communication.

[169] C.V. Shank, R. Yen, and C.V. Hirlimann, Phys. Rev. Lett. 50, 454 (1983).

[170] M.C. Downer, R.L. Fork, and C.V. Shank, J. Opt. Soc. Am. B 2, 595 (1985).

[171] H.W.K. Tom, G.D. Aumiller, and C.H. Brito-Cruz, Phys. Rev. Lett. $60,1438(1988)$.

[172] R.M. More, Laser Absorption in the Ultra-Short Pulse Domain ( Lawrence Livermore National Laboratory, 1993).

[173] B.-T.V. Vu, (unpublished) (1993).

[174] J. Heremans, et al., Phys. Rev. Lett. 60, 452 (1988). 
[175] O.L. Landen, D.G. Stearns, and E.M. Campbell, Phys. Rev. Lett. 63, 1475 (1989).

[176] H.M. Milchberg and R.R. Freeman, J. Opt. Soc. Am. B 6, 1351 (1989).

[177] R. Fedosejevs, et al., Phys. Rev. Lett. 64, 1250 (1990).

[178] X. Liu and D. Umstadter, Phys. Rev. Lett. 69, 1935 (1992).

[179] B. Chichkov and T. Peter, Z. Phys. D. 17, 123 (1990).

[180] B.-T.V. Vu, Time-resolved electron thermal conduction by probing of plasma formation in transparent solids with high power subpicosecond laser pulses. Ph.D. Dissertation (University of California, Davis, 1993).

[181] C. Rolland and P.B. Corkum, J. Opt. Soc. Am. B 3, 1625 (1986).

[182] P.B. Corkum, IEEE J. Quantum Electron. QE-21, 216 (1985).

[183] K.A. Goldberg, Prepulse Energy Suppression of Ultrashort Laser Pulses Using Second Harmonic Generation in Nonlinear Media. Ph.D. Dissertation (University of California, Berkeley, 1992).

[184] B. Nikolaus, D. Grischkowsky, and A.C. Balant, Opt. Lett. 8, 189 (1983).

[185] R.H. Stolen, J. Botineau, and A. Ashkin, Opt. Lett. 7, 512 (1982). 
[186] M. Ferray, et al., Opt. Commun. 75, 278 (1990).

[187] M.D. Perry, F.G. Patterson, and J. Weston, Opt. Lett. 15, 381 (1990).

[188] J.P. Heritage, et al., Appl. Phys. Lett. 47, 87 (1985).

[189] W.E. White, et al., Opt. Lett. 18, 1343 (1993).

[190] K. Yamakawa, C.P.J. Barty, H. Shiraga, and Y. Kato, IEEE J. Quantum Electron. QE-27, 288 (1991).

[191] D. Birx, et al., Appl. Phys. Lett. 32, 68 (1978).

[192] N.K. Moncur, Appl. Opt. 16, 1449 (1977).

[193] P.R. Bolton and A. Szoke, (private communication) .

[194] H.C. Kapteyn, M.M. Murnane, A. Szoke, and R.W. Falcone, Opt. Lett. 16, 490 (1991).

[195] M.M. Murnane, H.C. Kapteyn, and R.W. Falcone, Appl. Phys. Lett. 56,1948 (1990).

[196] M.D. Rosen, Femtosecond to nanosecond high-intensity lasers and applications, , E.M. Campbell, Editor. (SPIE, Bellingham, WA, 1990) p. 1660. 
[197] F. Salin, J. Squier, G. Mourou, and G. Vaillancourt, Opt. Lett. 16, 1964 (1991).

[198] S. Backus, et al., Opt. Lett. 18, 134 (1993).

[199] S.I. Anisimov, B.L. Kapeliovich, and T.L. Perel'man, Sov. Phys. JETP 39,375 (1974).

[200] R. Trebino, C.E. Barker, and A.E. Siegman, QE-22, 1413 (1986).

[201] R. Trebino, E.K. Gustafson, and A.E. Siegman, 3, 1295 (1986).

[202] P.L. Bhatnagar, E.P. Gross, and M. Krook, Phys. Rev. 94, 511 (1954).

[203] M. Abramowitz and I.A. Stegun, Handbook of Mathematical Functions.

Ninth Printing (Dover Publications, Inc., New York, 1964). 